University of Louisville

ThinkIR: The University of Louisville's Institutional Repository

Electronic Theses and Dissertations

$12-2012$

\title{
MR imaging of left-ventricular function : novel image acquisition and analysis techniques.
}

Hui Wang

University of Louisville

Follow this and additional works at: https://ir.library.louisville.edu/etd

\section{Recommended Citation}

Wang, Hui, "MR imaging of left-ventricular function : novel image acquisition and analysis techniques." (2012). Electronic Theses and Dissertations. Paper 1523.

https://doi.org/10.18297/etd/1523

This Doctoral Dissertation is brought to you for free and open access by ThinkIR: The University of Louisville's Institutional Repository. It has been accepted for inclusion in Electronic Theses and Dissertations by an authorized administrator of ThinkIR: The University of Louisville's Institutional Repository. This title appears here courtesy of the author, who has retained all other copyrights. For more information, please contact thinkir@louisville.edu. 


\title{
MR IMAGING OF LEFT-VENTRICULAR FUNCTION: NOVEL IMAGE ACQUISITION AND ANALYSIS TECHNIQUES
}

\author{
By \\ Hui Wang \\ M.S., EE, Shanghai Jiao Tong University, China, 2007
}

\begin{abstract}
A Dissertation
Submitted to the Faculty of the

J. B. Speed School of Engineering of the University of Louisville

in Partial Fulfillment of the Requirements

for the Degree of
\end{abstract}

Doctor of Philosophy

Department of Electrical and Computer Engineering

University of Louisville

December 2012 


\title{
MR IMAGING OF LEFT-VENTRICULAR FUNCTION: NOVEL IMAGE ACQUISITION AND ANALYSIS TECHNIQUES
}

\author{
Submitted by
}

Hui Wang

A Dissertation Approved on

$11 / 28 / 2012$

(Date)

by the Following Reading and Examination Committee: Amir A. Amini, Ph.D., Dissertation
Advisor

Aruni Bhatnagar, Ph.D.

Lijian Chen, Ph.D.

Hichem Frigui, Ph.D.

Tamer Inanc, Ph.D.

Jacek M. Zurada, Ph.D. 


\section{DEDICATION}

This dissertation is dedicated to my parents, Zeguo Wang and Guohua Zhai. 


\section{ACKNOWLEDGEMENTS}

I would like to express my deepest gratitude to my advisor Dr. Amir A. Amini for his guidance, support, and encouragement during my PhD study. Both the atmosphere in our lab and the wide range of research topics studied have provided an ideal and inspiring research environment. I would like to thank him for giving me the opportunity to work on interesting projects, for his guidance and intellectual interactions, opportunities that I was provided to meet prestigious scholars, and to attend several top conferences in the field of medical imaging. I am thankful for his encouragement to publish in top peer-viewed journals, edited books, and conference proceedings.

I would also like to thank all my committee members: Dr. Aruni Bhatnagar, Dr. Lijian Chen, Dr. Hichem Frigui, Dr. Tamer Inanc, and Dr. Jacek M. Zurada for useful suggestions and help. Special thanks go to Dr. Melanie Traughber and

Dr. Peter Martin at Philips Medical Systems for their support. I would like to pay special tribute to Dr. Stefan Fischer of Philips Medical Systems for the discussions and help that he gave me prior to his passing in June, 2011. He set an example for me for both his extensive knowledge of MRI at Philips and for his great personality.

I would like to thank all members of the Medical Imaging Laboratory (MIL) at the University of Louisville for their friendships and support. Special thanks go to Dr. Mohammadreza Negahdar who graduated in June, 2012 and is now at Stanford University for encouraging me with my research. Special thanks also go to Mo Kadbi for his help and support in every aspect.

I would like to thank all my friends at the University of Louisville: Dr. Weizhong Zhang, Dr. Lijun Zhang, Dr. Kan Liu, Yinan Cui, Shengpeng Jin, Shuang- 
shuang Jiang, to just name a few. Special thanks go to Dr. Dongqing Chen for his help and encouragement in both my academic research and personal life since the first day I came to the United States.

I would like to give my deepest appreciation to my parents, Zeguo Wang and Guohua Zhai for their true love and full belief in me that helped me get through all the difficult times. 


\title{
ABSTRACT \\ MR IMAGING OF LEFT-VENTRICULAR FUNCTION: NOVEL IMAGE ACQUISITION AND ANALYSIS TECHNIQUES
}

\author{
Hui Wang
}

November 28, 2012

Many cardiac diseases, such as myocardial ischemia, secondary to coronary artery disease, may be identified and localized through the analysis of cardiac deformations. Early efforts for quantifying ventricular wall motion used surgical implantation and tracking of radiopaque markers with X-ray imaging in canine hearts [1]. Such techniques are invasive and affect the regional motion pattern of the ventricular wall during the marker tracking process and, clearly are not feasible clinically. Noninvasive imaging techniques are vital and have been widely applied to the clinic.

MRI is a noninvasive imaging technique with the capability to monitor and assess the progression of cardiovascular diseases (CVD) so that effective procedures for the care and treatment of patients can be developed by physicians and researchers. It is capable of providing 3D analysis of global and regional cardiac function with great accuracy and reproducibility. In the past few years, numerous efforts have been devoted to cardiac motion recovery and deformation analysis from MR imaging sequences.

In order to assess cardiac function, there are two categories of indices that are used: global and regional indices. Global indices include ejection fraction, cavity volume, and myocardial mass [2]. They are important indices for cardiac disease 
diagnosis. However, these global indices are not specific for regional analysis. A quantitative assessment of regional parameters may prove beneficial for the diagnosis of disease and evaluation of severity and the quantification of treatment [3]. Local measures, such as wall deformation and strain in all regions of the heart, can provide objective regional quantification of ventricular wall function and relate to the location and extent of ischemic injury.

This dissertation is concerned with the development of novel MR imaging techniques and image postprocessing algorithms to analyze left ventricular deformations. A novel pulse sequence, termed Orthogonal CSPAMM (OCSPAMM), has been proposed which results in the same acquisition time as SPAMM for 2D deformation estimation while keeping the main advantages of CSPAMM [4,5]: i.e., maintaining tag contrast through-out the ECG cycle. Different from CSPAMM, in OCSPAMM the second tagging pulse orientation is rotated 90 degrees relative to the first one so that motion information can be obtained simultaneously in two directions. This reduces the acquisition time by a factor of two as compared to the traditional CSPAMM, in which two separate imaging sequences are applied per acquisition. With the application of OCSPAMM, the effect of tag fading encountered in SPAMM tagging due to $T_{1}$ relaxation is mitigated and tag deformations can be visualized for the entire cardiac cycle, including diastolic phases.

A multilevel B-spline fitting method (MBS) has been proposed which incorporates phase-based displacement information for accurate calculation of $2 \mathrm{D}$ motion and strain from tagged MRI $[6,7]$. The proposed method combines the advantages of continuity and smoothness of MBS, and makes use of phase information derived from tagged MR images. Compared to previous 2D B-spline-based deformation analysis methods, MBS has the following advantages: 1) It can simultaneously achieve a smooth deformation while accurately approximating the given data set; 2) Computationally, it is very fast; and 3) It can produce more accurate deformation results. 
Since the tag intersections (intersections between two tag lines) can be extracted accurately and are more or less distributed evenly over the myocardium, MBS has proven effective for $2 \mathrm{D}$ cardiac motion tracking. To derive phase-based displacements, 2D HARP and SinMod analysis techniques $[8,9]$ were employed. By producing virtual tags from HARP/SinMod and calculating intersections of virtual tag lines, more data points are obtained. In the reference frame, virtual tag lines are the isoparametric curves of an undeformed 2D B-spline model. In subsequent frames, the locations of intersections of virtual tag lines over the myocardium are updated with phase-based displacement. The advantage of the technique is that in acquiring denser myocardial displacements, it uses both real and virtual tag line intersections. It is fast and more accurate than 2D HARP and SinMod tracking.

A novel 3D sine wave modeling (3D SinMod) approach for automatic analysis of 3D cardiac deformations has been proposed [10]. An accelerated 3D complementary spatial modulation of magnetization (CSPAMM) tagging technique [11] was used to acquire complete $3 \mathrm{D}+\mathrm{t}$ tagged $\mathrm{MR}$ data sets of the whole heart (3 dynamic CSPAMM tagged MRI volume with tags in different orientations), in-vivo, in 54 heart beats and within 3 breath-holds. In 3D SinMod, the intensity distribution around each pixel is modeled as a cosine wave front. The principle behind 3D SinMod tracking is that both phase and frequency for each voxel are determined directly from the frequency analysis and the displacement is calculated from the quotient of phase difference and local frequency. The deformation fields clearly demonstrate longitudinal shortening during systole. The contraction of the LV base towards the apex as well as the torsional motion between basal and apical slices is clearly observable from the displacements. 3D SinMod can automatically process the image data to derive measures of motion, deformations, and strains between consecutive pair of tagged volumes in 17 seconds. Therefore, comprehensive 4D imaging and postprocessing for determination of ventricular function is now possible in under 10 minutes. For validation of 3D SinMod, 
$73 \mathrm{D}+\mathrm{t}$ CSPAMM data sets of healthy subjects have been processed. Comparison of mid-wall contour deformations and circumferential shortening results by 3D SinMod showed good agreement with those by 3D HARP. Tag lines tracked by the proposed technique were also compared with manually delineated ones. The average errors calculated for the systolic phase of the cardiac cycles were in the sub-pixel range. 


\section{TABLE OF CONTENTS}

\section{CHAPTER}

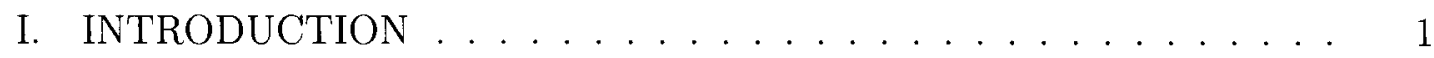

A. Heart Anatomy and Physiology . . . . . . . . . . . . 2

B. Stress and Strain . . . . . . . . . . . . . . . . . . 3

C. Dissertation Organization ................ . . 9

II. BASIC PRINCIPLES OF MRI . . . . . . . . . . . . . . . 11

A. MR Physics .............................. 11

1. Microscopic Perspective . . . . . . . . . . . . . 11

2. Macroscopic Perspective . . . . . . . . . . . . . . 14

3. Transverse Relaxation and Longitudinal Relaxation . . . . 16

4. Signal Detection . . . . . . . . . . . . . . 17

B. Magnetic Resonance Imaging . . . . . . . . . . . . 18

1. Slice Selection . . . . . . . . . . . . . . 18

2. Frequency Encoding . . . . . . . . . . . . . 20

3. Phase Encoding . . . . . . . . . . . . . . . . . . 21

4. Basic Imaging Pulse Sequences _. . . . . . . . . . . . 23

5. Image Acquisition Strategy _. . . . . . . . . . . . 24

III. MR TECHNIQUE FOR IMAGING CARDIAC FUNCTION . . . . . . 27

A. Cardiac Gating/Triggering . . . . . . . . . . . 28

B. Cine MR Imaging . . . . . . . . . . . . . . . . . . 29

C. MRI Tagging . . . . . . . . . . . . . . 32

1. Spatial Modulation of Magnetization (SPAMM) . . . . . 34 
2. Complementary Spatial Modulation of Magnetization $($ CSPAMM $) \ldots \ldots \ldots \ldots \ldots \ldots \ldots$

D. Phase-Contrast MRI . . . . . . . . . . . . . . . . . . 40

E. Displacement Encoding with Stimulated Echoes (DENSE) . . . 42

F. Strain Encoding (SENC) MRI . . . . . . . . . . . . 45

IV. REVIEW OF CARDIAC DEFORMATION ANALYSIS TECHNIQUES FROM MRI TAGGING . . . . . . . . . . . . . . . 48

A. Tracking Myocardial Beads/Landmark-based Methods . . . . . 48

B. Deformable Models . . . . . . . . . . . . . . . . . . . 50

C. Optical Flow Methods . . . . . . . . . . . . . . . . . . 52

D. Registration-based Methods . . . . . . . . . . . . . . 53

E. Frequency-based Methods . . . . . . . . . . . . . . . 54

1. Harmonic Phase Analysis (HARP) . . . . . . . . 54

2. Local Sine Wave Modeling . . . . . . . . . . . . . . . 59

3. Gabor Filter Banks . . . . . . . . . . . . . . . . . 61

F. Strain Pattern Analysis . . . . . . . . . . . . . . 62

V. 2D TAGGED MRI ANALYSIS WITH MULTILEVEL B-SPLINES . . 66

A. Multilevel B-Splines With Phase Information . . . . . . . 67

B. Results ........................ 71

VI. ORTHOGONAL CSPAMM (OCSPAMM) MR TAGGING FOR IMAGING VENTRICULAR WALL MOTION $\ldots \ldots \ldots \ldots$

A. Orthogonal CSPAMM Acquisition $\ldots \ldots \ldots \ldots$

B. Results . . . . . . . . . . . . . . . . . 82

VII. ANALYSIS OF 3D CSPAMM TAGGED MRI DATA WITH 3D SINMOD 85

A. Data Acquisition . . . . . . . . . . . . 85

B. 3D Sine Wave Modeling (3D SinMod) $\ldots \ldots \ldots$

C. Computational Time . . . . . . . . . . . . . . . . . . 91 
D. Validations . . . . . . . . . . . . . . . . . 94

1. Simulated Motion Fields . . . . . . . . . . . . . 95

2. Comparison with 3D HARP . . . . . . . . . 96

3. Comparison of Warped with Manually Delineated Tag Lines 97

E. Results . . . . . . . . . . . . . . . . . 100

1. Visualization of Motion Fields . . . . . . . . . 100

2. Circumferential Shortening . . . . . . . . . . 112

3. Strains . . . . . . . . . . . . . . . . . 127

VIII. CONCLUSIONS AND FUTURE DIRECTIONS . . . . . . . . . 134

REFERENCES . . . . . . . . . . . . . . . . . . . . 140

CURRICULUM VITAE . . . . . . . . . . . . . . . . . . 154 


\section{LIST OF TABLES}

TABLE 1. The values of the thirteen $k$ parameters of the cardiac simulator for the simulated data. . . . . . . . . . . . . . .

TABLE 2. Comparison of HARP, SinMod, and MBS on simulated data using the metric in equation (61). Distances are in pixels. . . . . . . 74

TABLE 3. Comparison of HARP, SinMod, and MBS on mid-ventricular systolic tagged data from a normal canine using the metric in Equation (61). Distances are in pixels. . . . . . . . . . . . .

TABLE 4. Average angle, length error and RRMS between ground truth motion field and motion field from HARP, SinMod, and MBS for simulated data. . . . . . . . . . . . . .

TABLE 5. RMS error between circumferential strains computed from the ground truth motion field and motion fields from HARP, SinMod, and MBS for simulated data. . . . . . . . . . . .

TABLE 6. Computational time for 3D SinMod algorithm on 7 in-vivo data sets. After zero-fill, each data set consisted of $20-24$ volumetric frames each with 112 voxels. . . . . . . . . . . . . .

TABLE 7. The average error between calculated motion fields and ground truth for simulated sequences with Gaussian noise added to the simulated data with standard deviation 0.05 . The reported errors

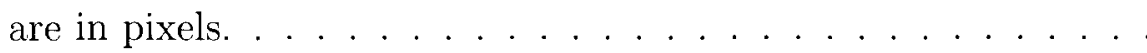

TABLE 8. The average error between deformed mid-wall contours from 3D SinMod and 3D HARP for slices 2 to 10 over all cardiac phases. The reported errors are in pixels. . . . . . . . . . . . . . 98 
TABLE 9. The average circumferential shortening error between results from 3D SinMod and 3D HARP for slices 2 to 10 over all cardiac phases.

The error is in percentage. . . . . . . . . . . . . . . . 112 


\section{LIST OF FIGURES}

FIGURE 1. Anatomy of the heart. The arrows show the direction of blood flow. This figure has been adapted from Texas Heart Institute [12]. 4

FIGURE 2. Coronary Circulation. This figure has been adapted from Texas Heart Institute $[12] \ldots \ldots \ldots \ldots \ldots$

FIGURE 3. Components of the strain tensor with respect to anatomic circumferential, longitudinal, and radial coordinates [13]. . . . . . 5

FIGURE 4 . The displacement from point $P$ to $p . \ldots \ldots \ldots$

FIGURE 5. Proton precessing around $B_{0} \ldots \ldots \ldots \ldots \ldots$

FIGURE 6. Microscopic magnetic moments align either in the parallel or antiparallel to the external magnetic field $B_{0}$. Since the number of protons in the parallel direction is slightly larger than the number in the anti-parallel direction, a small net magnetization $M$ is

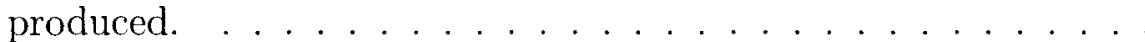

FIGURE 7. Longitudinal magnetization $M_{z}$ and transverse magnetization $M_{x y}$ after a RF pulse with $\alpha$ flip angle. . . . . . . . . .

FIGURE 8. Left: the magnetization vector $M$ precesses around the $\mathrm{x}^{\prime}$ axis in the rotating frame. Right: the actual motion of $\mathrm{M}$ is a spiral in the laboratory frame. . . . . . . . . . . . . . . .

FIGURE 9. Plot of frequency versus position along the direction of the sliceselection gradient $\vec{G}_{s s}$. The slope of $\vec{G}_{s s}$ represents the strength of the gradient. $\omega_{s}$ is the center frequency of excitation pulse. $\left[-\Delta \omega_{s}, \Delta \omega_{s}\right]$ is the frequency bandwidth. $d$ is the slice thickness. 
FIGURE 10. Diagram of spin echo sequence. $G_{s}$ is slice selection gradient. $G_{p}$ is phase encoding gradient. $G_{m}$ is frequency encoding gradient. [14] 24

FIGURE 11. Diagram of gradient echo sequence. $G_{s}$ is slice selection gradient. $G_{p}$ is phase encoding gradient. $G_{m}$ is frequency encoding gradient. $[14] \ldots \ldots \ldots \ldots \ldots \ldots \ldots \ldots \ldots \ldots \ldots \ldots \ldots \ldots$

FIGURE 12. Schematic diagram of ECG-gated breath-hold cine MRI. There are multiple cardiac phases in one cardiac cycle. For each phase, a segment is acquired in one heart beat. . . . . . . . . . .

FIGURE 13. A sequence of short-axis cine images acquired with b-FFE on a Philips Achieva 3T scanner. Ten out of thirty phases are shown from the top left to bottom right. The first row shows systolic images and the second row shows the diastolic images. Imaging parameters were as follows: $\mathrm{TE}=1.603 \mathrm{~ms}$, slice thickness $=$ $8 \mathrm{~mm}$, spatial resolution $=1.25 \times 1.25 \mathrm{~mm}^{2}$, acquisition matrix $=160 \times 187$, flip angle $=45^{\circ}$. A phased-array thoracic coil with 16 elements was used. . . . . . . . . . . . .

FIGURE 14. A sequence of long-axis cine images acquired with b-FFE on a Philips Achieva 3T scanner in the same volunteer whose shortaxis images were shown in Figure 13. Ten out of thirty phases are shown from the top left to bottom right. The first row shows systolic images and the second row shows diastolic images. Imaging parameters were as follows: $\mathrm{TE}=1.737 \mathrm{~ms}$, slice thickness $=$ $8 \mathrm{~mm}$, spatial resolution $=1.21 \times 1.21 \mathrm{~mm}^{2}$, acquisition matrix $=176 \times 211$, flip angle $=45^{\circ}$. A phased-array thoracic coil with 16 elements was used. . . . . . . . . . . . . . .

FIGURE 15. Tagging sequence timing scheme. Tagging pulse is usually applied at end-diastole, immediately after the R-wave. . . . . . . . . . 
FIGURE 16. SPAMM pulse sequence. Left: Timing diagram for SPAMM. RF $=$ radio-frequency excitation, $\mathrm{t}=$ time, $\mathrm{G}_{t}=$ wrap gradient for production of modulation. Letters a-d indicate corresponding time points in the right figure. Right: State of magnetization as a function of time. (a) Magnetization prior to initiation of the modulation sequence. (b) Magnetization after first RF pulse. (c) Magnetization after wrap (modulating) gradient pulse. (d) Longitudinal magnetization after second RF pulse. . . . . . .

FIGURE 17. The top row shows a sequence of short-axis mid-ventricular untagged images taken from a dog at three different time points in the cardiac cycle. (a) end-diastole, (b) mid-systole, and (c) endsystole. The bottom row shows tagged images at corresponding times and locations. . . . . . . . . . . . .

FIGURE 18. Timing diagram of a typical tagging experiment. A tagging sequence is applied before $t_{0}$ followed by a standard multiphase imaging sequence. $t_{k}$ corresponds to the start of the $k^{\text {th }}$ phase in the cardiac cycle. $\alpha^{k}$ corresponds to the RF pulse flip angle at $k^{\text {th }}$ phase in the cardiac cycle where $\alpha^{1}=\cdots \alpha^{k}=\cdots \alpha^{n}$. Note that each of the RF pulses $\alpha^{1}, \alpha^{2}, \cdots$ is applied multiple times corresponding to different strength of the phase encoding gradient. That creates a single image in the cine sequence. . . . . . . . .

FIGURE 19. Timing diagram of a 1-1 CSPAMM sequence. (a) Measurement with positive tagging pattern $T A G_{A}(x, y)$ (b) Measurement with negative tagging pattern $T A G_{B}(x, y) \ldots \ldots \ldots$ 
FIGURE 20. Visualization of magnetization subtraction in CSPAMM. The positive sinusoidal SPAMM tagging pattern is shown with solid lines and the negative sinusoidal SPAMM tagging pattern is shown with dashed lines. (a) Shows the magnetization in the initial state immediately after tagging pulse sequence. With time, longitudinal relaxation occurs. (b) Shows the magnetization after a certain time period. (c) Shows the CSPAMM tagging pattern which results from the subtraction of the negative pattern from the positive pattern in (b). $\ldots \ldots \ldots \ldots \ldots$

FIGURE 21. Visualization of k-space for an image modulated by a cosine in the horizontal direction. (a)k-space for one SPAMM with positive tagging pattern (b)k-space for the other SPAMM with negative tagging pattern (c)k-space for CSPAMM. . . . . . . . . .

FIGURE 22. Visualization of $\mathrm{k}$-space for an image modulated by a cosine in the vertical direction. (a)k-space for one SPAMM with positive tagging pattern (b)k-space for the other SPAMM with negative tagging pattern $(\mathrm{c}) \mathrm{k}$-space for CSPAMM. . . . . . . . . .

FIGURE 23. A bipolar gradient lobe. . . . . . . . . . . . . . . .

FIGURE 24. Timing diagram for DENSE in readout direction. Phase wrapping happens through the application of $G_{1} . G_{2}$ unwraps the phase [15]. 44

FIGURE 25. Timing diagram for SENC pulse sequence [16] . . . . . . . . 46

FIGURE 26. Tagged images, their Fourier peaks, and their corresponding phase

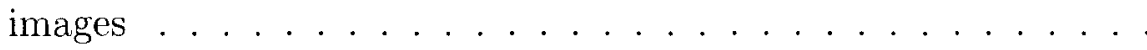

FIGURE 27. (a) An tagged MR image. (b) The magnitude of its Fourier transform. By extracting the spectral peak inside the circle in (b), a complex image is produced with a magnitude (c) and a phase (d). 55

FIGURE 28. Flow chart for the SinMod method . . . . . . . . . . . . . 61 
FIGURE 29. Overview of the proposed multilevel B-splines method . . . . . . 67

FIGURE 30. Multilevel B-spline Hierarchy . . . . . . . . . . . . . . . . . 69

FIGURE 31. Creating virtual tag lines and tag intersections. Column 1: virtual tag lines placed in the reference frame (solid green lines). Column 2: the motion field that warps the virtual tag lines (dashed blue

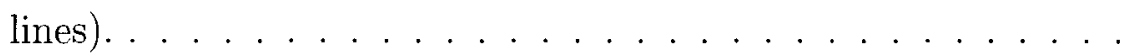

FIGURE 32. A realistic simulation. A 3-D solid model of the heart from Arts' Model at (a) end-diastole and (b) end-systole. Short-axis simulated images at (c) end-diastole and (d) end-systole. . . . . .

FIGURE 33. Tagged MR images and corresponding motion field between first two frames for both simulated and canine data. Canine data is from slice 4. (a)reference simulator image, (b) deformed simulator image, (c) motion field between (a) and (b), (d)reference canine image, (e)deformed canine image, (f) motion field between (d) and $(\mathrm{e}) \ldots \ldots \ldots \ldots \ldots 76 \ldots \ldots \ldots \ldots$

FIGURE 34. Spectrum of tagged images using (a) SPAMM and (b) CSPAMM. 80 FIGURE 35. Timing diagram for the OCSPAMM sequence. The first pair of $45^{\circ} \mathrm{RF}$ pulses with an interspersed tagging gradient are used to define the tags in $G_{x}$ direction. The second pair of $45^{\circ} \mathrm{RF}$ pulses with an interspersed tagging gradient orthogonal to the first tagging gradient are used to define the tags in $G_{y}$ direction. A TFEEPI sequence is used for imaging as shown in Figure 36. . . . . .

FIGURE 36. A timing diagram of the TFE-EPI sequence used for imaging the modulated magnetization with EPI factor 5. After each RF pulse, five k-space profiles are acquired with the help of the blip gradients in phase encoding direction. . . . . . . . . . . . . . . 
FIGURE 37. Visualization of k-space for the OCSPAMM sequence. (a) k-space for tagged image with positive tagging pattern (b) k-space for tagged image with negative tagging pattern in orthogonal direction to (a) (c) k-space for OCSPAMM. . . . . . . . . . . .

FIGURE 38. Phantom components. (a) Contracting cardiac phantom (b) Rotating cardiac phantom (c) Air pump . . . . . . . . . . .

FIGURE 39. Seven Images from fourteen cardiac phases for a rotating phantom using the proposed OCSPAMM pulse sequence. Every other frame during an entire cardiac cycle is shown. The order is from top-left to bottom-right. . . . . . . . . . . .

FIGURE 40. Seven images of fourteen cardiac phases during an entire cardiac cycle for a rotating phantom using SPAMM pulse sequence. Every other frame is shown. The order is from top-left to bottom-right. In comparison to images acquired with OCSPAMM from the same phantom (Figure 39), tag fading is clearly evident. . . . . . . .

FIGURE 41. Seven Images from fourteen cardiac phases for a contracting phantom using the proposed OCSPAMM pulse sequence. Every other frame during an entire cardiac cycle is shown. The order is from top-left to bottom-right. . . . . . . . . . . . . . .

FIGURE 42. Illustration of 3D CSPAMM tagging data acquisition for one cardiac phase. Solid lines represent tagging planes, while dashed lines represent imaging planes. (a) Short Axis (SA) stack, (b) First Long Axis (LA1) stack, and (c) Second Long Axis (LA2) stack. (d) A 2D slice from (a). (e) A 2D slice from (b). (f) A 2D slice from $(\mathrm{c}) . \ldots \ldots \ldots \ldots \ldots \ldots$ 
FIGURE 43. Example mid-ventricular slices for three data sets with orthogonal tagging directions from 3D CSPAMM sequence. (a) At the beginning of systole. (b) At end-systole. (c) At end-diastole. The first row is from the SA view, and the second and third rows are from two LA views. . . . . . . . . . . . . . . . .

FIGURE 44. Short axis slices from apex to base acquired with the 3D CSPAMM sequence. (a) The first phase (at the beginning of systole). (b) The 6th phase (at mid-systole). (c) The 11th phase (at endsystole). (d). The 16th phase (at mid-diastole). (e) The 20th phase (at end-diastole). Row 1: slice 1 (apex). Row 2: slice 3 . Row 3: slice 5. Row 4: slice 7. Row 5: slice 9. Row 6: slice 11. Row 7 : slice 13 (basal). . . . . . . . . . . . . . .

FIGURE 45. Flow chart of the 3D SinMod algorithm to obtain 3D displacements. Two stacks of 3D image volumes at times $n$ and $n+1$ are Fourier transformed. A 3D bandpass filter is applied to separate one off-center spectral peak. A window around the band-passed frequencies is inverse Fourier transformed to obtain down-sampled complex volume J. Further analysis renders a down-sampled map of displacement $\mu$. This map is upsampled to the initial size of the volume. Repeating the same algorithms for the two LA directions recovers full 3D displacements. . . . . . . . . . . . . . .

FIGURE 46. Illustration of Butterworth bandpass filter transfer function in 1D. In this case, cutoff frequency $\omega_{\text {cutoff }}=0.1, \omega_{c}=0.125$, and $n=5.93$ 
FIGURE 47. Displacement fields in the simulated sequence. (a) Translation displacement field in the y direction. (b) Non-homogeneous translations in $\mathrm{x}, \mathrm{y}, \mathrm{z}$ directions. (c) Contraction displacement field. The first row displays 3D visualization, while the second row is the $2 \mathrm{D}$ projection of the first row. . . . . . . . .

FIGURE 48. Flow diagram for comparison of mid-wall contour deformation using motion field from 3D SinMod algorithm with 3D HARP. .

FIGURE 49. Mid-wall contours at a mid-ventricular slice for all phases. Traversing from top-left to the bottom-right are phases 1 to 20 . The red contours are deformed with motion field from 3D SinMod. The green contours are deformed with motion field from 3D HARP. .

FIGURE 50. 3D mid-wall contours tracked by 3D SinMod. (a) At the beginning of systole. (b) At end-systole. (c) At end-diastole. . . . . . . . . 99

FIGURE 51. End systolic 3D motion field for slice 1, 3, 6, and 10 for data set 1.100 FIGURE 52. End systolic 3D motion field for slice 1, 3, 6, and 10 for data set 2.101 FIGURE 53. End systolic 3D motion field for slice 1, 3, 6, and 10 for data set 3.101 FIGURE 54. End systolic 3D motion field for slice 1, 3, 6, and 10 for data set 4.102 FIGURE 55. End systolic 3D motion field for slice 1, 3, 6, and 10 for data set 5.102 FIGURE 56. End systolic 3D motion field for slice 1, 3, 6, and 10 for data set 6.103 FIGURE 57. End systolic 3D motion field for slice 1, 3,6, and 10 for data set 7.103 FIGURE 58. End systolic motion field on slices 1, 3, 5, 7, 9, 10 for data set 1 (row 1: apex, row 2: mid-ventricular, row 3: base). . . . . . . 104

FIGURE 59. End systolic motion field on slices 1, 3, 5, 7, 9, 10 for data set 2 (row 1: apex, row 2: mid-ventricular, row 3: base). . . . . . 105 FIGURE 60 . End systolic motion field on slices 1, 3, 5, 7, 9, 10 for data set 3 (row 1: apex, row 2: mid-ventricular, row 3: base). . . . . . 106 
FIGURE 61. End systolic motion field on slices 1, 3, 5, 7, 9, 10 for data set 4 (row 1: apex, row 2: mid-ventricular, row 3: base) . . . . . 107

FIGURE 62. End systolic motion field on slices 1, 3, 5, 7, 9, 10 for data set 5 (row 1: apex, row 2: mid-ventricular, row 3: base). . . . . 108

FIGURE 63. End systolic motion field on slices 1, 3, 5, 7, 9, 10 for data set 6 (row 1: apex, row 2: mid-ventricular, row 3: base). . . . . . . 109

FIGURE 64. End systolic motion field on slices 1, 3, 5, 7, 9, 10 for data set 7 (row 1: apex, row 2: mid-ventricular, row 3: base) . . . . . . . 110

FIGURE 65. 3D SinMod's average error as a function of time for determining tag line displacements during systole for 7 in-vivo data sets. Results for data sets $1,2,3,4,5,6$, and 7 are differentiated with red, green, black, yellow, blue, cyan, and magenta colors. Please note that the error for each time point was calculated from the error between warped tag line locations and manually delineated tag lines on all image slices. Also, note that the manually delineated tag lines at the reference frame were warped to all subsequent frames and results were compared with the actual location of tag lines. This explains the reason for the increased error in later phases. . . . . . . . . . . . . . . .

FIGURE 66. Circumferential Shortening (Equation 69) at different phases during the cardiac cycle for data set 1 . Traversing from top-left to the bottom-right are results for slices 2 to $7 \ldots \ldots$. . . . 113

FIGURE 67. Circumferential Shortening (Equation 69) at different phases during the cardiac cycle for data set 1 . Traversing from top-left to the bottom-right are results for slices 8 to $10 \ldots \ldots \ldots$. . . . 114 
FIGURE 68. Circumferential Shortening (Equation 69) at different phases during the cardiac cycle for data set 2 . Traversing from top-left to the bottom-right are results for slices 2 to $7 \ldots \ldots \ldots 115$

FIGURE 69. Circumferential Shortening (Equation 69) at different phases during the cardiac cycle for data set 2. Traversing from top-left to the bottom-right are results for slices 8 to $10 \ldots \ldots$. . . . . 116

FIGURE 70. Circumferential Shortening (Equation 69) at different phases during the cardiac cycle for data set 3 . Traversing from top-left to the bottom-right are results for slices 2 to $7 \ldots \ldots \ldots$

FIGURE 71. Circumferential Shortening (Equation 69) at different phases during the cardiac cycle for data set 3 . Traversing from top-left to the bottom-right are results for slices 8 to $10 \ldots \ldots \ldots \ldots$

FIGURE 72. Circumferential Shortening (Equation 69) at different phases during the cardiac cycle for data set 4 . Traversing from top-left to the bottom-right are results for slices 2 to 7 . . . . . . . . . . 119

FIGURE 73. Circumferential Shortening (Equation 69) at different phases during the cardiac cycle for data set 4 . Traversing from top-left to the bottom-right are results for slices 8 to $10 \ldots \ldots$. . . . .

FIGURE 74. Circumferential Shortening (Equation 69) at different phases during the cardiac cycle for data set 5 . Traversing from top-left to the bottom-right are results for slices 2 to $7 \ldots \ldots \ldots$. . .

FIGURE 75. Circumferential Shortening (Equation 69) at different phases during the cardiac cycle for data set 5 . Traversing from top-left to the bottom-right are results for slices 8 to $10 \ldots \ldots$. . . . .

FIGURE 76. Circumferential Shortening (Equation 69) at different phases during the cardiac cycle for data set 6 . Traversing from top-left to the bottom-right are results for slices 2 to $7 \ldots \ldots \ldots$ 
FIGURE 77. Circumferential Shortening (Equation 69) at different phases during the cardiac cycle for data set 6 . Traversing from top-left to the bottom-right are results for slices 8 to $10 \ldots \ldots$. . . . . 124

FIGURE 78. Circumferential Shortening (Equation 69) at different phases during the cardiac cycle for data set 7 . Traversing from top-left to the bottom-right are results for slices 2 to $7 \ldots \ldots \ldots$

FIGURE 79. Circumferential Shortening (Equation 69) at different phases during the cardiac cycle for data set 7 . Traversing from top-left to the bottom-right are results for slices 8 to $10 \ldots \ldots$. . . . . 126

FIGURE 80. Geometrical representation of the regional divisions for strain analysis for both the left and right ventricles in the LA view [17]. 128

FIGURE 81. Geometrical representation of the regional divisions for strain analysis for both the left and right ventricles in the SA view. Illustrated are (a) the basal region, (b) the mid-cavity region, and (c) the apical region $[17] \ldots \ldots \ldots \ldots \ldots$

FIGURE 82. Histogram of the Jacobian of deformation (see Equation 13) as calculated from 3D SinMod for different data sets. Note that the Jacobian of the deformation gradient tensor for all myocardial points in space and time for each of the $3 \mathrm{D}+\mathrm{t}$ data sets ( 10 slices and 10 temporal frames) are summarized in a single histogram. Traversing from top-left to the bottom-right are results for data sets 1 to $4 \ldots \ldots \ldots \ldots \ldots \ldots \ldots \ldots \ldots \ldots \ldots \ldots$ 
FIGURE 83. Histogram of the Jacobian of deformation (see Equation 13) as calculated from 3D SinMod for different data sets. Note that the Jacobian of the deformation gradient tensor for all myocardial points in space and time for each of the $3 \mathrm{D}+\mathrm{t}$ data sets $(10$ slices and 10 temporal frames) are summarized in a single histogram. Traversing from top-left to the bottom-right are results for data sets 5 to $7 \ldots \ldots \ldots \ldots \ldots \ldots \ldots \ldots \ldots \ldots \ldots \ldots \ldots$

FIGURE 84 . The average radial, circumferential, and longitudinal strain curves during systole for all 7 data sets. Radial strain is shown in dotted blue line. Circumferential strain is shown in dashed green line. Longitudinal strain is shown in solid red line. Traversing from top-left to the bottom-right are results for basal anterior (segment 1), basal antero-septal (segment 2), basal infero-septal (segment 3), basal inferior (segment 4), basal posterior (segment 5), basal lateral $($ segment 6$) \ldots \ldots \ldots \ldots \ldots \ldots$

FIGURE 85 . The average radial, circumferential, and longitudinal strain curves during systole for all 7 data sets. Radial strain is shown in dotted blue line. Circumferential strain is shown in dashed green line. Longitudinal strain is shown in solid red line. Traversing from top-left to the bottom-right are results for mid-ventricular anterior (segment 7), mid-ventricular antero-septal (segment 8), mid-ventricular infero-septal (segment 9), mid-ventricular inferior (segment 10), mid-ventricular posterior (segment 11), midventricular lateral $($ segment 12$) \ldots \ldots \ldots$. . . . . . . . 132 
FIGURE 86 . The average radial, circumferential, and longitudinal strain curves during systole for all 7 data sets. Radial strain is shown in dotted blue line. Circumferential strain is shown in dashed green line. Longitudinal strain is shown in solid red line. Traversing from top-left to the bottom-right are results for apical anterior (segment 13), apical septal (segment 14), apical inferior (segment 15), apical lateral (segment 16), and apex (segment 17) . . . . . . 133 


\section{CHAPTER I INTRODUCTION}

Globally, cardiovascular diseases (CVD) are the number one cause of death and are projected to remain so. An estimated 17 million people died from CVD in 2005 , representing $30 \%$ of all global deaths. Of these deaths, 7.2 million were due to heart attacks and 5.7 million were due to stroke. If current trends continue, by 2030 an estimated 23.6 million people will die from cardiovascular diseases in the world [18]. In America, an estimated 80 million adults (more than one in three) have one or more types of cardiovascular diseases. In 2005, about 864,000 people died of CVD, accounting for $35.3 \%$ of all deaths [19].

MRI can provide three-dimensional analysis of global and regional cardiac function with great accuracy and reproducibility [20]. Compared to ultrasound and $\mathrm{X}$-ray $\mathrm{CT}$, the advantages of MRI are: 1) It is noninvasive and uses non-ionizing radiation; 2) It has 3D and 4D imaging capabilities with high spatial and temporal resolutions and with good soft tissue contrast; 3) It can image the heart at arbitrary orientations; 4) It is valuable in diagnosing a broad range of conditions; 5) It is able to evaluate both the structure and function of the heart. There are also some disadvantages to MRI: it is not real-time, it is expensive, and compared to X-ray CT and Ultrasound, has lower resolution.

Many approaches have been proposed for tracking cardiac motion and for computing deformation parameters and mechanical properties of the heart from a variety of cardiac MR imaging techniques. This chapter briefly introduces heart anatomy (Section I.A) and two important concepts in heart mechanics, namely, strain and stress (Section I.B). 


\section{A. Heart Anatomy and Physiology}

The heart is a muscular cone-shaped organ located in the upper body between the lungs [12]. The essential function of the heart is to pump blood around the body. The heart is divided into separate right and left sections by the interventricular septum, as shown in Figure 1. Each of these (right and left) sections is also divided into upper and lower compartments known as atria and ventricles, respectively. The four chambers for the mammalian heart are: right atrium (RA), right ventricle (RV), left atrium (LA), and left ventricle (LV). The two atria act as collecting reservoirs for blood returning to the heart while the two ventricles act as pumps to eject the blood to the body. Deoxygenated blood returns to the heart via the major veins (superior and inferior vena cava), enters the right atrium, passes into the right ventricle, and from there is ejected into the pulmonary artery on the way to the lungs. Oxygenated blood returning from the lungs enters the left atrium via the pulmonary veins, passes into the left ventricle, and is then ejected into the aorta. As in any pumping system, there are four valves to prevent the back flow of blood. The tricuspid valve is between the right atrium and right ventricle. The pulmonic valve is between the right ventricle and pulmonary artery. The mitral valve is between the left atrium and left ventricle and the aortic valve is between the left ventricle and the aorta.

Cardiac cycle refers to the events of one complete heart beat. In normal adults, the length of the cardiac cycle is usually about 0.8 second. The cardiac cycle consists of two phases: systole and diastole. During diastole the ventricles are filled and the atria contract. Then during systole, the ventricles contract while the atria are relaxed and filled. In detail, the pumping action starts with the simultaneous contraction of the two atria. This contraction gives an added push to get the flood into the ventricles during diastole. Shortly after that, the ventricles contract, signifying the beginning of systole. The aortic and pulmonic valves open and blood is ejected from the ventricles, while the mitral and tricuspid valves close to prevent back flow. At 
the same time, the atria start to fill with blood again. After a while, the ventricles relax, the aortic and pulmonic valves close, the mitral and tricuspid valves open, and the ventricles start to fill with blood again, signifying the end of systole and the beginning of diastole. In a normal ECG waveform over an R-R interval, the P wave indicates the activation of atria, corresponding to their contraction. The QRS complex indicates the activation of the ventricles. Both P and QRS are known as depolarization waves. $\mathrm{T}$ wave indicates the ventricular recovery (repolarization wave). Systole covers the period from the onset of QRS complex to the end of T wave. The remaining part of a cardiac cycle is diastole. In cardiac MRI, where imaging typically requires synchronization with the ECG, the pulse sequence is triggered when the amplitude of the $\mathrm{R}$ wave reaches its maximum. This can be determined either by threshold detection or peak-slope detection.

Another important concept is the coronary circulation [12], from which the heart receives the energy and nourishment it needs (Figure 2), which resides on the epicardial (outer) surface of the heart. Coronary circulation refers to the blood circulation in the vessels of the heart muscle. Because the myocardium is very thick, it requires coronary blood vessels to deliver blood deep into it. The aorta branches off into two main coronary blood vessels: the right coronary artery (RCA) and the left coronary artery (LCA) which divides into left anterior descending and circumflex branches. These vessels deliver oxygen-rich blood to the myocardium.

\section{B. Stress and Strain}

The primary function of the heart is fundamentally mechanical [13]. The basic measurements of myocardial mechanics are the three-dimensional stresses and strains, which depend on position and orientation in the myocardium and vary in time throughout the cardiac cycle. The terms "strain" and "stress" are often used together in cardiology. But they are different physical quantities with distinct units. Stress is 


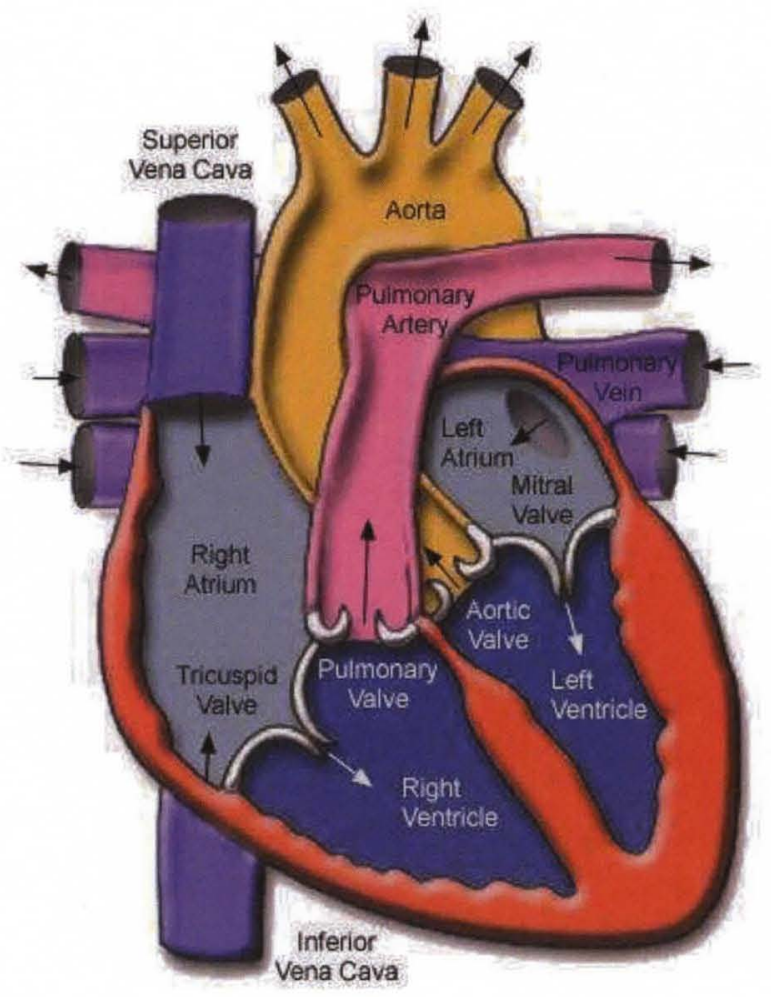

FIGURE 1 -Anatomy of the heart. The arrows show the direction of blood flow. This figure has been adapted from Texas Heart Institute [12]. 


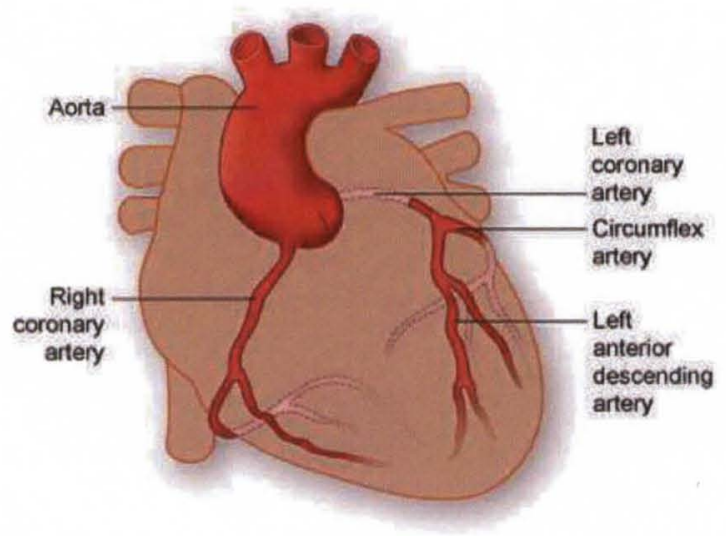

FIGURE 2-Coronary Circulation. This figure has been adapted from Texas Heart Institute [12].

defined as force per unit area, so it has the same unit $F / m^{2}$ as pressure. In cardiology, stress is interaction forces acting across surfaces between adjacent regions of muscle, while strain represents the change of shape at any point in the wall between the original reference state and the subsequent deformed state, which is dimensionless [13].

The matrix components of the stress and strain tensors depend on the selected frame of reference. It is conventional to choose the orthogonal system, such as the local circumferential, longitudinal, and radial axes, as shown in Figure 3.
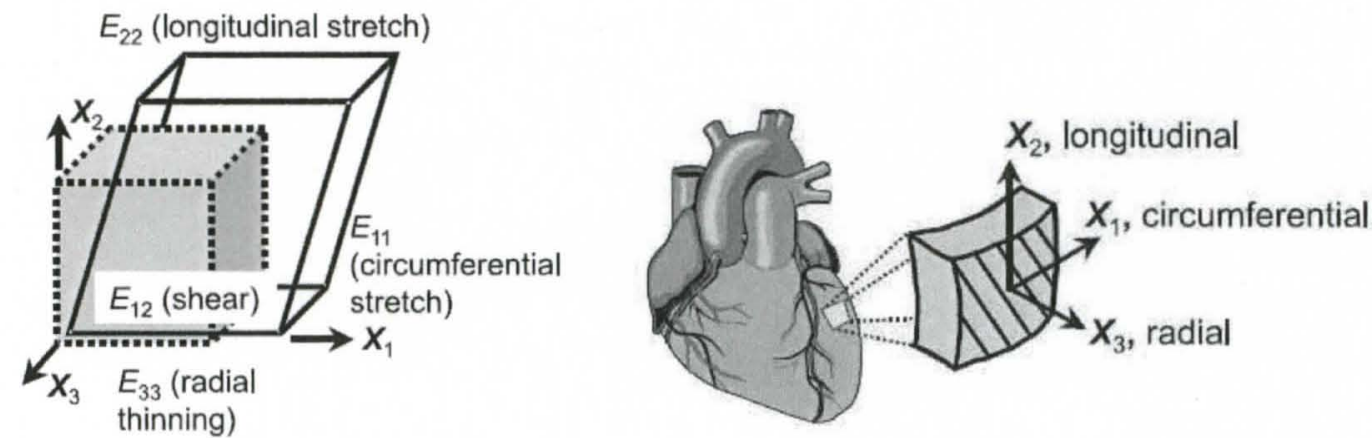

FIGURE 3-Components of the strain tensor with respect to anatomic circumferential, longitudinal, and radial coordinates [13]. 
1. Normal (Axial) Stress: stress that acts perpendicular to a surface. It can be either compressional or tensional.

2. Shear Stress: stress that acts parallel to a surface. It can cause one object to slide over another. It also tends to deform originally rectangular objects into parallelograms. The most general definition is that shear acts to change the angles in an object.

Ventricular wall stress and strain are inhomogeneous. Their components can change significantly from place to place in the myocardium. This heterogeneity makes understanding ventricular mechanics a significant challenge, but also gives tagged MRI an opportunity to stand out from other techniques in measuring strains. Development of tagged MRI was a major milestone in the field of cardiac mechanics. Tagged MRI technique will be discussed in detail later.

Regional myocardial stress and strain have direct or indirect relationship with cardiac diseases. At present, regional strain distribution measurement is much more practical than the stress measurement. For measuring systolic function, end-diastole is a conventional unstrained reference state. An illustration of the relationship between the initial (initial, reference, and undeformed configurations are interchangeable throughout this section) and deformed configuration is shown in Figure 4. Suppose a material point at position $\mathbf{X}\left(X_{1}, X_{2}, X_{3}\right)$ in the undeformed solid moves to a new position $\mathbf{x}\left(x_{1}, x_{2}, x_{3}\right)$ when the solid is loaded. A mapping $\mathbf{x}=\chi(\mathbf{X}, t)$ would describe the motion. The displacement of the material point $\mathbf{u}$ is

$$
\mathbf{u}(t)=\mathbf{x}(t)-\mathbf{X}
$$

One measurement of the deformation in the reference configuration is the length change of a segment $d X$ at point $X$ :

$$
d \mathbf{x}=\chi(\mathbf{X}+d \mathbf{X})-\chi(\mathbf{X}) \approx[\nabla \chi(\mathbf{X})] \cdot d \mathbf{X}=F \cdot d \mathbf{X}
$$




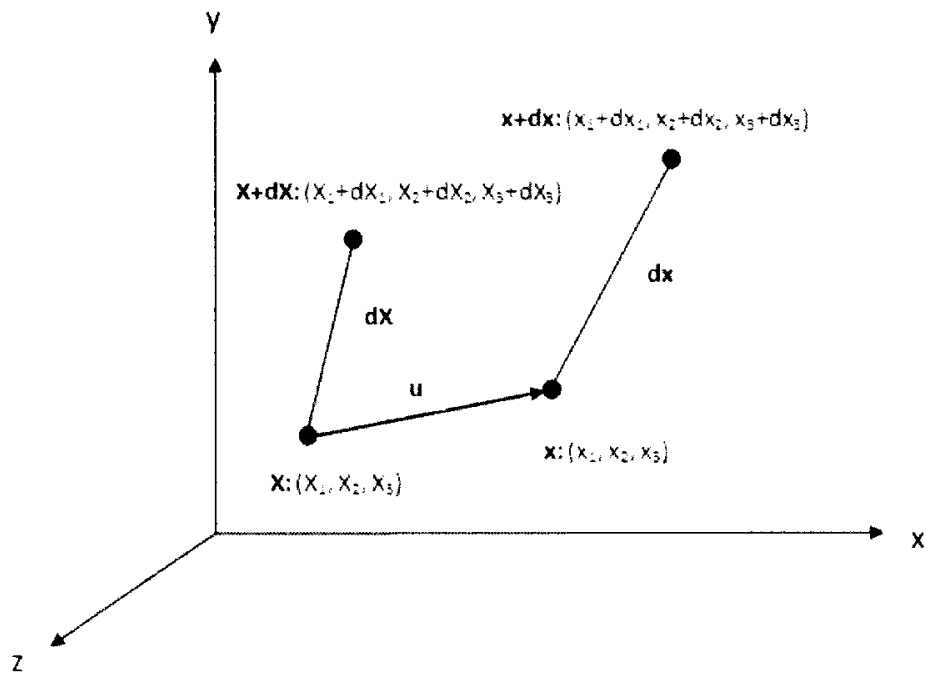

FIGURE 4 - The displacement from point $P$ to $p$.

where $\nabla$ is gradient operator and $F$ is the deformation gradient at $\mathbf{X}$. According to chain rule, the relationship between $d \mathbf{x}$ in deformed configuration and $d \mathbf{X}$ in reference configuration is:

$$
d x_{i}=\frac{\partial x_{i}}{\partial X_{j}} d X_{j}
$$

The mapping in the above equation is called deformation gradient tensor (DGT) $F_{i j}$.

$$
F_{i j}=\frac{\partial x_{i}}{\partial X_{j}}
$$

The matrix form of deformation gradient tensor is:

$$
\mathbf{F}=\left[\begin{array}{ccc}
F_{11} & F_{12} & F_{13} \\
F_{21} & F_{22} & F_{23} \\
F_{31} & F_{32} & F_{33}
\end{array}\right]=\left[\begin{array}{ccc}
\frac{\partial x_{1}}{\partial X_{1}} & \frac{\partial x_{1}}{\partial X_{2}} & \frac{\partial x_{1}}{\partial X_{3}} \\
\frac{\partial x_{2}}{\partial X_{1}} & \frac{\partial x_{2}}{\partial X_{2}} & \frac{\partial x_{2}}{\partial X_{3}} \\
\frac{\partial x_{3}}{\partial X_{1}} & \frac{\partial x_{3}}{\partial X_{2}} & \frac{\partial x_{3}}{\partial X_{3}}
\end{array}\right]
$$

From Equation (1),

$$
x_{i}=X_{i}+u_{i}
$$

Taking the derivative of the above equation with respect to $X_{1}, X_{2}$, and $X_{3}$ and 
writing in matrix form in terms of the displacement field $\mathbf{u}, F$ becomes

$$
\mathbf{F}=\left[\begin{array}{ccc}
1 & 0 & 0 \\
0 & 1 & 0 \\
0 & 0 & 1
\end{array}\right]+\left[\begin{array}{ccc}
\frac{\partial u_{1}}{\partial X_{1}} & \frac{\partial u_{1}}{\partial X_{2}} & \frac{\partial u_{1}}{\partial X_{3}} \\
\frac{\partial u_{2}}{\partial X_{1}} & \frac{\partial u_{2}}{\partial X_{2}} & \frac{\partial u_{2}}{\partial X_{3}} \\
\frac{\partial u_{3}}{\partial X_{1}} & \frac{\partial u_{3}}{\partial X_{2}} & \frac{\partial u_{3}}{\partial X_{3}}
\end{array}\right]
$$

To find a measure of the change in length of $d \mathbf{X}$, strain is defined as:

$$
\begin{aligned}
\varepsilon=\frac{1}{2} \frac{|d \mathbf{x}|^{2}-|d \mathbf{X}|^{2}}{|d \mathbf{X}|^{2}} & =\frac{1}{2} \frac{d \mathbf{x}^{T} d \mathbf{x}-d \mathbf{X}^{T} d \mathbf{X}}{|d \mathbf{X}|^{2}} \\
& =\frac{1}{2} \frac{(\mathbf{F} d \mathbf{X})^{T}(\mathbf{F} d \mathbf{X})-d \mathbf{X}^{T} d \mathbf{X}}{|d \mathbf{X}|^{2}} \\
& =\frac{d \mathbf{X}^{T} \frac{1}{2}\left(\mathbf{F}^{T} \mathbf{F}-I\right) d \mathbf{X}}{|d \mathbf{X}|^{2}}
\end{aligned}
$$

Lagrangian strain tensor (also called Green's strain tensor) $\mathbf{E}$ is often used to characterize the infinitesimal deformation at a point.

$$
\mathbf{E}=\frac{1}{2}\left(\mathbf{F}^{T} \mathbf{F}-I\right)
$$

The normal strain in the direction of the unit vector $n$ can be calculated from the Lagrangian strain tensor through the quadratic form

$$
n^{T} \mathbf{E} n
$$

where $n$ may point to any direction on the unit sphere. Due to the geometry of the ventricle, the normal strains are usually calculated in radial, circumferential, and longitudinal directions (See Figure 3).

Since $F$ is nonsingular, the inverse map, $\mathbf{F}^{-1}$, exists and allows for the Eulerian description of strain which is given by

$$
\begin{aligned}
\varepsilon=\frac{1}{2} \frac{|d \mathbf{x}|^{2}-|d \mathbf{X}|^{2}}{|d \mathbf{x}|^{2}} & =\frac{1}{2} \frac{d \mathbf{x}^{T} d \mathbf{x}-d \mathbf{X}^{T} d \mathbf{X}}{|d \mathbf{x}|^{2}} \\
& =\frac{1}{2} \frac{d \mathbf{x}^{T} d \mathbf{x}-\left(\mathbf{F}^{-1} d \mathbf{x}\right)^{T} \mathbf{F}^{-1} d \mathbf{x}}{|d \mathbf{x}|^{2}} \\
& =\frac{d \mathbf{x}^{T} \frac{1}{2}\left(I-\left(\mathbf{F} \mathbf{F}^{T}\right)^{-1}\right) d \mathbf{x}}{|d \mathbf{x}|^{2}}
\end{aligned}
$$


where $\mathbf{G}$ is often called the Eulerian strain tensor which is defined as follows.

$$
\mathbf{G}=\frac{1}{2}\left(I-\left(\mathbf{F F}^{T}\right)^{-1}\right)
$$

Since there is one-to-one correspondence between material points during continuous deformation, the determinant of $F$, normally referred to as the Jacobian, has to be nonzero:

$$
J=\operatorname{det}(F)
$$

In the absence of any deformation the Jacobian is equal to unity. Since the deformation is assumed to be a continuous function of time, it is impossible for the Jacobian to be negative without passing through 0 which was previously excluded from physical possibility. Therefore, the Jacobian must be greater than 0 for a continuous deformation to be physically possible.

Regional myocardial strains have direct or indirect relationship with cardiac diseases. Most of the motion tracking and analysis methods aim to extract strains in the heart, for the reason that strain encapsulates the basic mechanical function of the myocardium and has clinical potential. This dissertation is concerned with the development of novel MR imaging techniques and image postprocessing algorithms to analyze left ventricular deformations.

\section{Dissertation Organization}

The dissertation is organized as follows. Chapter I has briefly introduced the heart anatomy and two important concepts in cardiac mechanics: stress and strain. Chapter II covers the basic principles of MRI. Chapter III explains the principles of cardiac MRI techniques for imaging ventricular deformations including cine MRI, tagged MRI including SPAMM, CSPAMM and 3D CSPAMM, phase contrast MRI, Displacement encoding with stimulated echoes (DENSE), and Strain encoding (SENC). Chapter IV reviews existing cardiac tagged MR image analysis methods. 
Chapter V explains multilevel B-spline (MBS) technique for 2D cardiac motion tracking using tagged MRI. Chapter VI introduces a novel acquisition technique called OCSPAMM. A novel 3D cardiac deformation analysis technique based on 3D sine wave modeling (3D SinMod) highly applicable to 3D CSPAMM acquisition is proposed and comprehensively validated in Chapter VII. Conclusions and future research plans are given in Chapter VIII. 


\section{CHAPTER II BASIC PRINCIPLES OF MRI}

The clinical motivations and the significance of this dissertation were given in the previous chapter. In this chapter, the basic principles of Magnetic Resonance Imaging are explained which are foundations for the entire thesis. A detailed description of MR physics is given first followed by the image acquisition process, known as MR imaging.

\section{A. MR Physics}

The first successful nuclear magnetic resonance experiment in condensed matter was carried out by Purcell [21] and Bloch [22] independently, in 1946. These experiments laid the foundations for MRI. The magnetic resonance phenomenon can be described by both microscopic and macroscopic perspectives. In this section, both approaches are used for clarity. The microscopic perspective can better explain fundamental behavior of magnetization. Once the fundamentals are understood, the formation of MR signal is more easily described by macroscopic perspective. Please refer to $[14,23-25]$ for more details about the theory and principles of MRI.

\section{Microscopic Perspective}

The most commonly used source of signal in MRI is from ${ }^{1} H$, protons. Each proton is a charged particle with angular momentum. A spinning proton can classically be represented with a dipole magnetic field. Within a strong magnetic field $B_{0}$, the magnetic moment of each proton will precess around the axis of the static $B_{0}$ 


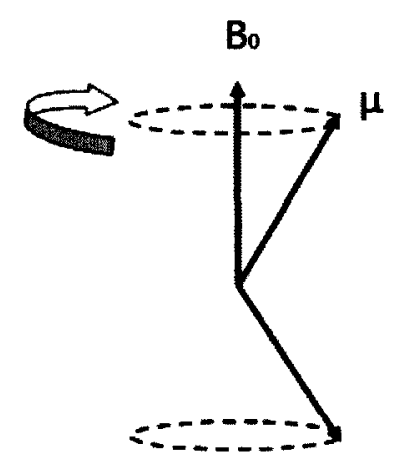

FIGURE 5 - Proton precessing around $B_{0}$

field (See Figure 5). Frequency of precession is proportional to the strength of $B_{0}$. Resonance frequency (or Larmor frequency or precession frequency) in radians/s can be represented as:

$$
\omega_{0}=\gamma B_{0}
$$

Larmor frequency in $\mathrm{Hz}$ is:

$$
f_{0}=\frac{\gamma B_{0}}{2 \pi}
$$

where $\gamma$ is called the gyromagnetic ratio, which is different for different nuclei. For ${ }^{1} H, \frac{\gamma}{2 \pi}=42.58 \mathrm{MHz} / \mathrm{T}$.

The net magnetization of the sample can be considered the summation of all proton magnetic moments in this sample. In the absence of a $B_{0}$ field, all the ${ }^{1} H$ nuclei in tissues are in random orientations. Therefore, the microscopic magnetic moments cancel each other and the net magnetic moment (macroscopic magnetization) is 0 . In the presence of a $B_{0}$ field, $B_{0}$ will cause a torque to make the magnetic moment of the spins (microscopic magnetization) align with $B_{0}$. But unlike a compass under the influence of earth's magnetic field, the microscopic magnetic moment $\mu$ will never exactly align with $B_{0}$. The protons align in two configurations: with the magnetic moment aligned either parallel (low energy state) or anti-parallel (higher energy state) to the large static $B_{0}$ field, as shown in Figure 6. The number of protons in the parallel state is slightly larger than those in the anti-parallel state resulting in the 

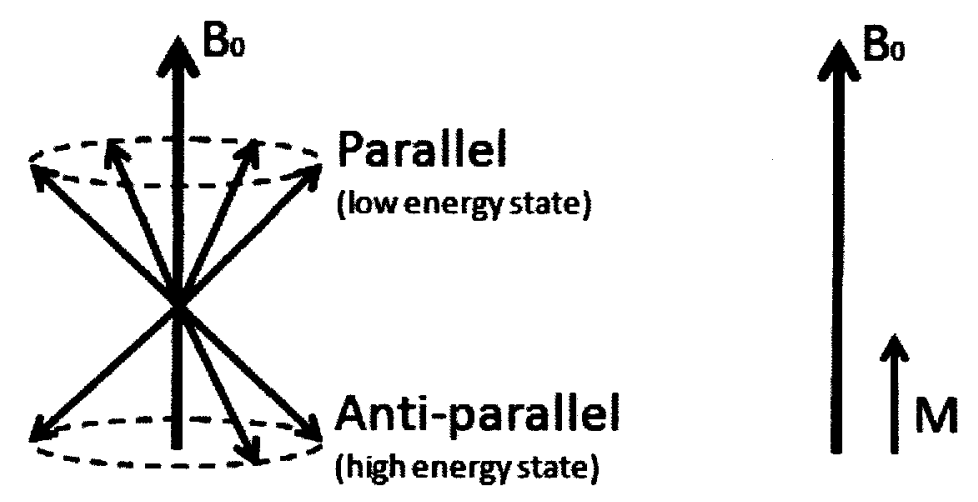

FIGURE 6-Microscopic magnetic moments align either in the parallel or anti-parallel to the external magnetic field $B_{0}$. Since the number of protons in the parallel direction is slightly larger than the number in the anti-parallel direction, a small net magnetization $\mathrm{M}$ is produced.

net magnetization to point in the direction of $B_{0}$. Note that because the precessing nuclear magnetic dipole moments have random phases, the transverse components of the dipole moments sum to zero. $M$ is the source of the NMR signal.

The strength of the microscopic magnetic moment for any nucleus is

$$
|\vec{u}|=\gamma \frac{\hbar}{2 \pi}[I(I+1)]^{1 / 2}
$$

where $\hbar$ is the planck's constant. $I$ is the spin quantum number which depends on the number of protons and neutrons in the nucleus. $I \neq 0$ only if mass number (number of neutrons plus protons) is odd or atomic number (number of protons) is odd or both. For ${ }^{1} H,|\vec{u}|=\frac{\gamma \hbar \sqrt{3}}{4 \pi}$. While all protons in ${ }^{1} H$ are in the same energy level with $B_{0}=0$, when $B_{0} \neq 0$, Zeeman splitting occurs $\left(E=-\mu_{z} B_{0}\right)$. For the parallel state, $E=-\frac{\gamma \hbar B_{0}}{4 \pi}$, and for anti-parallel $E=\frac{\gamma \hbar B_{0}}{4 \pi}$.

In order to obtain NMR signal, transition must be induced between protons in the parallel and anti-parallel states. Since $\Delta E=\frac{\gamma \hbar B_{0}}{2 \pi}=\hbar f_{0}$ between the parallel and anti-parallel state and $f=63.9 \mathrm{MHz}$ at $1.5 \mathrm{~T}$, matching electromagnetic energy (EM) at the resonance frequency is in the radio frequency (RF) range. External energy is deposited through application of a short-duration RF pulse. The factors 
that affect net magnetization are shown in equation (17). Larger $\gamma$, larger $B_{0}$, and lower temperature will lead to larger net magnetization. The net magnetization is in the z-direction with the transverse magnetization being zero. The larger the net magnetization, the larger the MR signal.

$$
M_{0}=\sum \mu=\frac{\gamma \hbar}{4 \pi} \times\left(N_{p}-N_{a}\right)=\frac{\gamma \hbar}{4 \pi} \times\left(N_{s} \frac{\gamma \hbar B_{0}}{4 \pi K T}\right)
$$

where $N_{p}$ and $N_{a}$ are the number of spins in the parallel and anti-parallel states. $N_{s}=N_{p}-N_{a}, K$ is the Boltzmann coefficient $=1.38 \times 10^{-23} \mathrm{~J} /$ Kelvin and $T$ is absolute temperature in Kelvins.

\section{Macroscopic Perspective}

In the microscopic perspective, effect of RF pulse is to move spins from the lower energy state to the higher energy state (parallel to anti-parallel). In the vector model, this corresponds to the transverse magnetization $M_{x y}$ having a non-zero value. A detectable NMR signal is only possible from components of transverse magnetization. Therefore, the longitudinal magnetization, $M_{z}$, needs to be rotated into the $x y$ plane. By applying current through RF coils surrounding the sample, the spin system can be deliberately excited and thereby the behavior of $M$ can be controlled. In other words, the spin system is excited using RF pulses so that the stimulated system will in turn induce RF signals as output. In order to rotate $M_{0}$ into the $x y$ plane, a second field $B_{1}$ is turned on which is perpendicular in direction of $z$-axis. $B_{1}$ is produced by the RF coil. In addition to precessing around $B_{0}$ at frequency $w=\gamma B_{0}, M_{0}$ will concurrently start to precess around $B_{1}$ at frequency $\omega_{1}=\gamma B_{1}$. To an observer, it appears that $M_{z}$ is rotating (or tipping away) from the $z$-axis. The angle of rotation around the $x$-axis in this case is called the flip angle $\alpha$ and is dependent on how long $B_{1}$ is turned on as well as its strength. Because the RF excitation pulse changes $\alpha$, such a pulse is referred as an $\alpha$-pulse. 


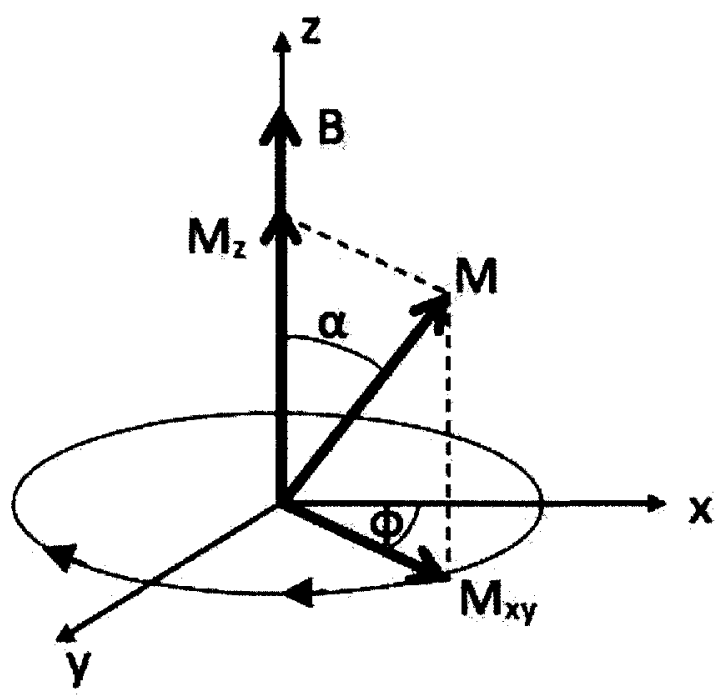

FIGURE 7 -Longitudinal magnetization $M_{z}$ and transverse magnetization $M_{x y}$ after a RF pulse with $\alpha$ flip angle.

After an $\alpha$-pulse, the magnetization $M$ can be decomposed into two components: longitudinal magnetization $M_{z}$ and transverse magnetization $M_{x y}$, as shown in Figure 7. $M_{z}$ is in the same direction as the main magnetic field $B_{0}$ and $M_{x y}$ is the component $M$ on a plane that is orthogonal to the $z$ direction. Mathematically, their relationships are:

$$
\begin{aligned}
& M_{z}=M_{0} \cos \alpha \\
& M_{x}=M_{0} \sin \alpha \cos (-\gamma B t+\phi) \\
& M_{y}=M_{0} \sin \alpha \sin (-\gamma B t+\phi)
\end{aligned}
$$

For simplicity, transverse components can be combined through the complex exponential as

$$
M_{x y}=M_{0} \sin \alpha e^{j(-\gamma B t+\phi)}
$$

The standard fixed Cartesian frame is also called the laboratory frame. The rotating frame of reference is one in which the $z^{\prime}$ axis is in the $B_{0}$ direction but the $x^{\prime}$ and $y^{\prime}$ axes rotate around the $z^{\prime}$ axis at Larmor frequency. This is shown in Figure 8. 

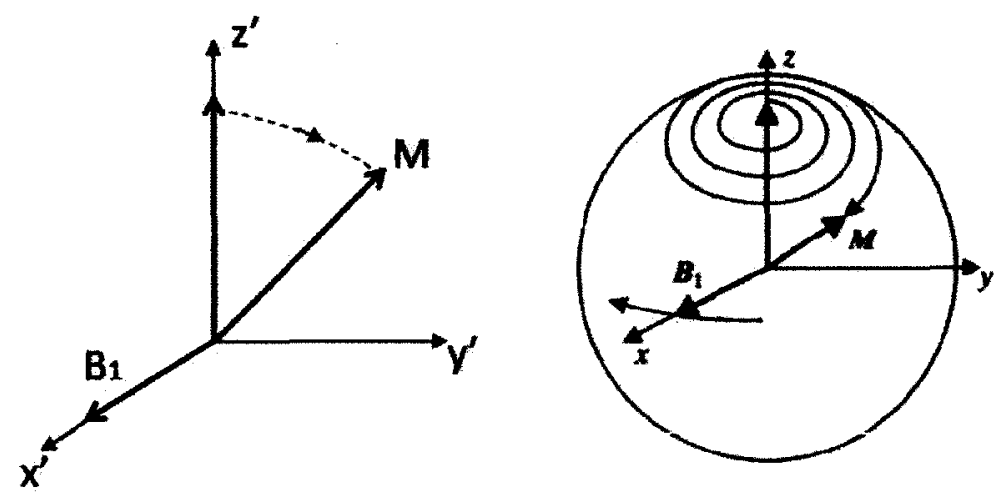

FIGURE 8-Left: the magnetization vector $M$ precesses around the $x^{\prime}$ axis in the rotating frame. Right: the actual motion of $\mathrm{M}$ is a spiral in the laboratory frame.

3. Transverse Relaxation and Longitudinal Relaxation

As explained in the previous section, after the RF pulse ( $B_{1}$ field) is turned on, the transverse magnetization $\left|M_{x y}\right|$ becomes non-zero. In the microscopic perspective, the reason is that $\sum \mu_{x y}$ for the spins starts to become nonzero: individual spins start to precess in phase. They will develop phase coherence. Block equations describe the behavior of $\overrightarrow{M(t)}=\left(M_{x}, M_{y}, M_{z}\right)$ with time. When $B_{1}$ is turned off, spins relax back to its equilibrium state, that is, $M_{x}, M_{y}$ to $0, M_{z}$ to $M_{0}$ in the vector model. In general, after $\alpha$-pulse, the transverse relaxation satisfies

$$
M_{x y}(t)=M_{0} \sin \alpha e^{-j\left(\omega_{0} t-\phi\right)} e^{-t / T_{2}}
$$

In the rotating frame,

$$
M_{x y}(t)=M_{0} \sin \alpha e^{j \phi} e^{-t / T_{2}}
$$

The time constant for decay of transverse magnetization is called $T_{2}$ relaxation. The physical basis for $T_{2}$ relaxation is loss of phase coherence among spins (spin-spin interactions) precessing in the transverse plane. Even in perfectly homogeneous $B_{0}$ field, spins precess at slightly different frequencies since each can experience a slightly different magnetic field due to their interactions with neighboring nuclei. Recall that $\frac{d \phi}{d t}=\omega_{0}$ is the relation between phase and precessional angular velocity, the Larmor 
frequency. In addition to phase incoherence which will occur even in a perfectly homogeneous $B_{0}$ field, resulting in decay of $\left|M_{x y}\right|$ by $T_{2}$, in practice, inhomogeneities (non-uniformities) in the $B_{0}$ field cause decay of $\left|M_{x y}\right|$ by $T_{2}^{*}$ with $T_{2}^{*}<T_{2}$. The decay due to $B_{0}$ field inhomogeneities is reversible via application of $180^{\circ} \alpha$-pulse. Decay of $\left|M_{x y}\right|$ due to pure $T_{2}$ effects is irreversible.

Longitudinal relaxation, also called $T_{1}$ relaxation, governs the process of return of spins from high energy state to the low energy state. The physical basis for $T_{1}$ relaxation involves protons loosing their energy to the surrounding lattice in the process of relaxing back to the low energy (parallel) state. Recovery of longitudinal magnetization is a result of $T_{1}$ relaxation after an $\alpha-$ pulse. In fact, this is precisely why the net magnetization needs to be tipped into the transverse plane. Once the net magnetization is tipped following application of an $\alpha$-pulse and the $\alpha$-pulse is turned off, and before $M_{x y} \rightarrow 0$, a free induction decay (FID) signal is recorded.

$$
M_{z}(t)=M_{z}\left(0^{+}\right) e^{-t / T_{1}}+M_{0}\left(1-e^{-t / T_{1}}\right)=M_{0} \cos \alpha e^{-t / T_{1}}+M_{0}\left(1-e^{-t / T_{1}}\right)
$$

If $\alpha=\pi / 2$,

$$
M_{z}(t)=M_{0}\left(1-e^{-t / T_{1}}\right)
$$

Different tissues have different values for $T_{1}$ and $T_{2}$. For most tissues, $5 T_{2}<$ $T_{1}<10 T_{2}$ and $250 \mathrm{~ms}<T_{1}<2500 \mathrm{~ms}$. Transverse magnetization is long gone before longitudinal magnetization fully recovers. Generally, in tissues with molecules that are small and fast moving, $T_{1}$ and $T_{2}$ are larger.

\section{Signal Detection}

In order to detect the emitted signal, an RF coil is placed close to the object. Farady's Law states that when the magnetic flux enclosed by a loop of wire changes with time, current is produced in the loop, thus inducing a voltage. In fact, this is precisely why the net magnetization needs to be tipped into transverse plane. The 
free induction decay (FID) signal however is from the entire tissue area. NMR signal need to be distinguished in local groups of voxels in order to make an image.

\section{B. Magnetic Resonance Imaging}

In 1973, Lauterbur [26] proposed the use of magnetic field gradients for spatial localization of NMR signals which laid the foundation for magnetic resonance imaging. When the gradient fields are turned on, the precessional frequencies of the spins become linearly dependent on their spatial locations. The frequency and the phase of the precessing magnetization are measured by the RF receiver coil. The basic approach to MRI performs slice selection, phase encoding, and frequency encoding multiple times to make an image.

\section{Slice Selection}

In the presence of a gradient field $\vec{G}$ with components $\left(G_{x}, G_{y}, G_{z}\right)$, the total magnetic strength that a proton at position $\vec{r}=(x, y, z)$ experiences in $\hat{z}$ direction and at time $t$ can be expressed as:

$$
\begin{aligned}
B_{z}(x, y, z, t) & =\left(B_{0}+G_{x}(t) x+G_{y}(t) y+G_{z}(t) z\right) \hat{z} \\
& =\left(B_{0}+\vec{G} \cdot \vec{r}\right) \hat{z}
\end{aligned}
$$

The gradient field $\vec{G}$ creates a net positive and negative magnetic environment that adds to and subtracts from the main magnetic field $B_{0}$. Associated with the local change in magnetic field is a local change in precessional frequencies. Transmission of an RF pulse tuned to Larmor frequency of a planar tissue slice when gradients on will only create transverse magnetization for protons in that slice. This is called selective excitation. Changing constant frequency $\omega_{s}$ of the RF pulse will shift slice location to different position in the patient. If an RF pulse is applied at frequency $\omega_{s}$ with excitation bandwidth of $\pm \Delta \omega_{s}$, protons precessing at frequency between $\omega_{s}-\Delta \omega_{s}$ and 


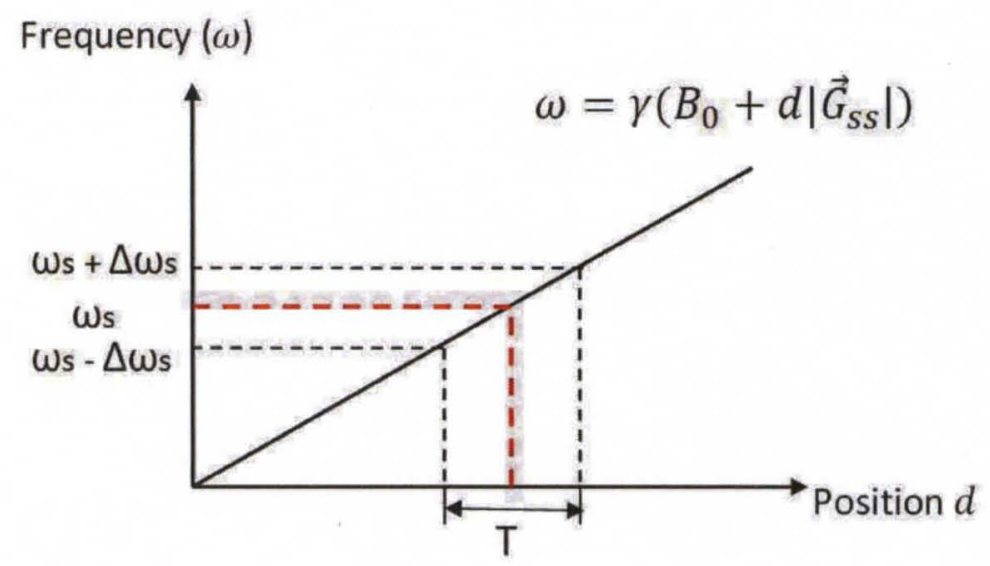

FIGURE 9 - Plot of frequency versus position along the direction of the slice-selection gradient $\vec{G}_{s s}$. The slope of $\vec{G}_{s s}$ represents the strength of the gradient. $\omega_{s}$ is the center frequency of excitation pulse. $\left[-\Delta \omega_{s}, \Delta \omega_{s}\right]$ is the frequency bandwidth. $d$ is the slice thickness.

$\omega_{s}+\Delta \omega_{s}$ will be excited. Changing RF bandwidth $\Delta \omega_{s}$ or changing gradient strength $\left|\vec{G}_{s s}\right|$ can change slice thickness. Equation (27) shows the relationship between them (See Figure 9).

$$
T=\frac{2 \Delta \omega_{s}}{\gamma\left|\vec{G}_{s s}\right|}
$$

During slice selection, since protons precess at different frequencies in slice direction, different protons accumulate different phases depending on their position within the slice thickness. By solving the Bloch equations, it can be shown that accumulated phase is

$$
\phi_{S L}(d)=\gamma\left|\vec{G}_{s s}\right| d \tau / 2
$$

for an RF pulse of duration $\tau$. To eliminate the extra phase, a rephasing (refocusing) gradient $\vec{G}_{s s}^{\text {ref }}$ is applied immediately following $\vec{G}_{s s}$ but with opposite polarity for time period $\tau^{r e f}=\tau / 2$. Thus total phase will be zero for all spins across the slice. All spins will precess coherently and an FID may be measured.

Following an $\alpha$-excitation, recall Equation (22), the transverse relaxation for 
a uniform sample (e.g., in a test tube) and uniform $B_{0}$ is:

$$
M_{x y}(t)=M_{0} \sin \alpha e^{-j\left(\omega_{0} t-\phi\right)} e^{-t / T_{2}}
$$

In reality, $M_{x y}$ varies with $x, y$, and $t$ :

$$
M_{x y}^{\prime}(x, y, t)=M_{0}(x, y) \sin \alpha e^{-j\left(\omega_{0} t-\phi\right)} e^{-t / T_{2}(x, y)}
$$

Assuming the slice is very thin with no $\mathrm{z}$ dependence,

$$
S(t)=\iint M_{x y}^{\prime}(x, y, t) d x d y=A \iint M_{0}(x, y) e^{-t / T_{2}(x, y)} d x d y e^{-j \omega_{0} t}
$$

If effective spin density is defined as $g(x, y)=A M_{0}(x, y) e^{-t / T_{2}(x, y)}$, the corresponding baseband demodulated signal becomes

$$
S_{0}(t)=e^{j \omega_{0} t} S(t) \approx \iint g(x, y) d x d y
$$

It is constant for small t. After slice selection, there is no position dependence of $S_{0}(t)$ other than selective excitation of the slice location.

\section{Frequency Encoding}

Frequency encoding is achieved by a frequency-encoding gradient on the imaged object. This gradient causes the Larmor frequencies to be spatially dependent in that direction - known as frequency-encoding or read-out direction. Therefore, time-domain NMR signals will consist of a range of frequencies, each corresponding to a different spatial location.

$$
f(x)=\frac{\gamma}{2 \pi}\left(B_{0}+G_{x} x\right)=f_{0}+\frac{\gamma}{2 \pi} G_{x} x
$$

Therefore, $S(t)$ in Equation (29) and $S_{0}(t)$ in Equation (30) need to be rewritten as

$$
\begin{aligned}
S(t) & =A \iint M_{0}(x, y) e^{-t / T_{2}(x, y)} e^{-j 2 \pi\left(f_{0}+\frac{\gamma}{2 \pi} G_{x} x\right) t} d x d y \\
S_{0}(t) & =\iint g(x, y) e^{-j 2 \pi\left(\frac{\gamma}{2 \pi} G_{x} t\right) x} d x d y
\end{aligned}
$$


where $S_{0}(t)$ is the recorded baseband signal after slice selection when the read-out gradient has been turned on.

Letting $k_{x}=\frac{\gamma}{2 \pi} G_{x} t$ be the spatial frequency variable in the $\mathrm{x}$ direction and $k_{y}$ be the spatial frequency in y direction,

$$
S_{0}(t)=S_{0}\left(\frac{2 \pi k_{x}}{\gamma G_{x}}\right)=G\left(k_{x}, k_{y}=0\right)
$$

is the Fourier transform of $g(x, y)$ along one line $\left(k_{y}=0\right)$. Applying a more general gradient during readout will lead to

$$
\begin{gathered}
f(x, y)=\frac{\gamma}{2 \pi}\left(B_{0}+G_{x} x+G_{y} y\right) \\
S_{0}(t)=\iint g(x, y) e^{-j 2 \pi\left(\frac{\gamma}{2 \pi} G_{x} x+\frac{\gamma}{2 \pi} G_{y} y\right) t} d x d y
\end{gathered}
$$

where $k_{x}=\frac{\gamma}{2 \pi} G_{x} t$ and $k_{y}=\frac{\gamma}{2 \pi} G_{y} t$. With both gradients on at the same time, every acquisition becomes a polar line in k-space. By varying the gradient strength of $G_{x}$ and $G_{y}$, k-space lines for a large number of $\theta^{\prime} s$ are collected. Filtered-back projection reconstruction can then be used to reconstruct the data.

\section{Phase Encoding}

Instead of performing frequency-encoding in two directions to generate polar rays to fill the $\mathrm{k}$-space, it is possible to perform rectilinear scanning of $\mathrm{k}$-space with phase encoding in one direction and frequency encoding in the orthogonal direction. A gradient is turned on in the phase encoding (PE) direction (perpendicular to slice select and frequency encode direction, e.g., $y$ direction) with strength $G_{y}$, prior to read out for a time period typically referred to as phase-encoding time, $\tau_{P E}$, and subsequently is switched off. During $\tau_{P E}$, the protons within the slice precess at frequency $\omega_{y}=\gamma G_{y} y$. At time $t=\tau_{P E}$ when the $\mathrm{PE}$ gradient is switched off, the 
accumulated phase in $y$ direction is:

$$
\Phi_{y}(y)=\int_{0}^{\tau_{P E}} \omega_{y} d t=\omega_{y} \tau_{P E}=\gamma G_{y} y \tau_{P E}
$$

The net effect is that a $y$-dependent (assuming $y$ is the PE direction) phase is introduced in the slice. In this case, following phase and frequency encoding, the final baseband signal may be expressed as

$$
S_{0}(t)=\iint g(x, y) e^{-j 2 \pi\left(\frac{\gamma}{2 \pi} G_{x} x t\right)} e^{-j 2 \pi\left(\frac{\gamma}{2 \pi} G_{y} y \tau_{P E}\right)} d x d y
$$

Let $k_{x}=\frac{\gamma}{2 \pi} G_{x} t$ as before and $k_{y}=\frac{\gamma}{2 \pi} G_{y} \tau_{P E}$

$$
S_{0}(t)=\iint g(x, y) e^{-j 2 \pi\left(k_{x} x+k_{y} y\right)} d x d y=G\left(k_{x}, k_{y}\right)
$$

To fill the k-space in a rectilinear fashion, one would perform $n$ phase encoding steps; each time, $S_{0}(t)$ corresponds to a different value of the phase-encoding gradient. An inverse two-dimensional Fourier transform $F_{2 D}^{-1}\left\{G\left(k_{x}, k_{y}\right)\right\}$ converts the Fourier representation into the spatial representation in order to produce the MR image.

How densely the $\mathrm{k}$-space is sampled determines the spatial extent, also known as the "field of view" or FOV for short. How far out the k-space goes determines the spatial resolution in the image. The corresponding relationships are as follows:

$$
\begin{aligned}
F O V_{x} & =\frac{2 \pi}{\gamma G_{x}} f_{s}=\frac{1}{\Delta k_{x}} \\
F O V_{y} & =\frac{2 \pi}{\gamma G_{y}} \frac{1}{\tau_{P E}}=\frac{1}{\Delta k_{y}} \\
k x_{\max } & =\frac{1}{\Delta x} \\
k y_{\max } & =\frac{1}{\Delta y}
\end{aligned}
$$

Note that no anti-aliasing filter is applied in the phase-encode direction. If tissue exists outside of $F O V_{y}$ range, it will cause wrap-around artifact in the PE direction which in turn means that $\Delta k_{y}$ (and therefore $\Delta G_{y}$ ) should be reduced. If $\mathrm{k}$-space is subsampled, foldover or aliasing occurs. 


\section{Basic Imaging Pulse Sequences}

A pulse sequence (also called imaging sequence, pulse program) is a series of events comprising RF pulses, gradients, and data acquisition to produce an image. Two basic pulse sequences are spin echo (SE) sequence and gradient echo (GE) sequence.

The spin echo sequence is made up of a series of events: $90^{\circ}$ pulse, $180^{\circ}$ rephasing pulse at $t=T E / 2$, and signal reading at $t=T E$. The pulse sequence timing diagram for a basic spin echo sequence is shown in Figure 10. The first $90^{\circ}$ pulse applied while $G_{s}$ is turned on, creates transverse component of magnetization for slice of interest (slice excitation). Phase encoding gradient is applied. To cover negative $k_{x}$ frequencies, only a gradient pulse with a positive polarity is applied in the frequency-encode (read-out) direction. The subsequent $180^{\circ}$ pulse effectively reverses the $k_{x}$ values to $-\left(k_{x}\right)_{\max }$ prior to read out. The $180^{\circ}$ is also frequency selective and is applied simultaneously with $G_{s}$ at the half of the echo time (TE/2). This series is repeated at each time interval $T R$ (Repetition time). With each repetition, a k-space line is filled, thanks to a different phase encoding. Spin echo sequences generally result in images with high contrast to noise ratio (CNR) and signal to noise ratio (SNR). Disadvantage is that it needs longer acquisition time. The repetition time $(T R)$ between two consecutive RF excitation pulses must be long enough to allow sufficient recovery of longitudinal magnetization via $T_{1}$ relaxation.

Gradient echo is also called field echo, gradient-recalled echo, gradient-refocused echo (GRE). Gradient reversal on the readout direction forms the echo. The pulse sequence timing diagram for a basic gradient echo sequence is shown in Figure 11. A readout prephasing gradient lobe dephases the spin isochromats and then they are rephased by a readout gradient with opposite polarity. The flip angle $\alpha$ for gradient echo is usually smaller. $T R$ is usually in the range of $2-50 \mathrm{~ms}$. Excitation pulse is the only RF pulse in each $T R$ interval. GRE can be classified by transverse mag- 


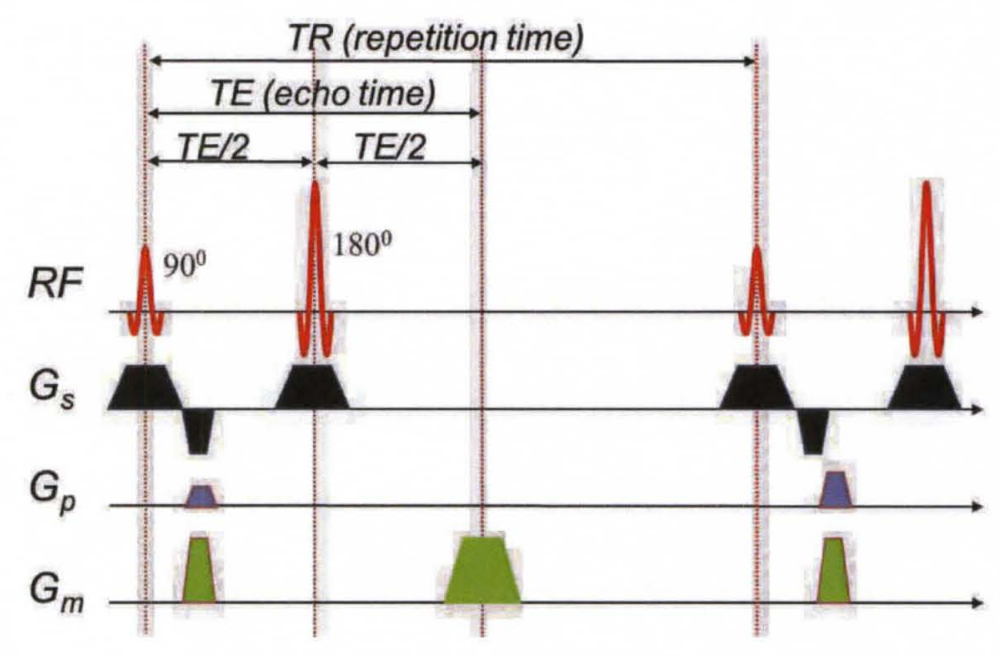

FIGURE 10 -Diagram of spin echo sequence. $G_{s}$ is slice selection gradient. $G_{p}$ is phase encoding gradient. $G_{m}$ is frequency encoding gradient. [14]

netization $M_{x y}$. If $M_{x y}=0$ right before excitation pulse, it is called spoiled GRE. If $M_{x y} \neq 0$ right before excitation pulse, it belongs to Steady State Free Precession (SSFP).

\section{Image Acquisition Strategy}

1D MR imaging, line scan, is rarely used today except for the applications such as signal tracking and line-scan diffusion imaging. 2D imaging, also named planar or slice imaging, involves two main steps: slice selection and spatial encoding within the selected slice. Multislice 2D imaging can be performed in two ways. One is called sequential acquisition, i.e, filling all k-space lines for one slice before moving to the next one. To improve SNR, if signal averaging is desired, it is typically performed before moving on to the next k-space line. The other way is interleaved acquisition, i.e, obtaining a specific $\mathrm{k}$-space line for multiple slices in $T R$ and then repeating next k-space line for all slices. Signal averaging is usually performed before moving to the next k-space line. It is mostly common used in the gradient echo pulse sequence. The good thing about interleaved acquisition is that the idle time in a sequential 


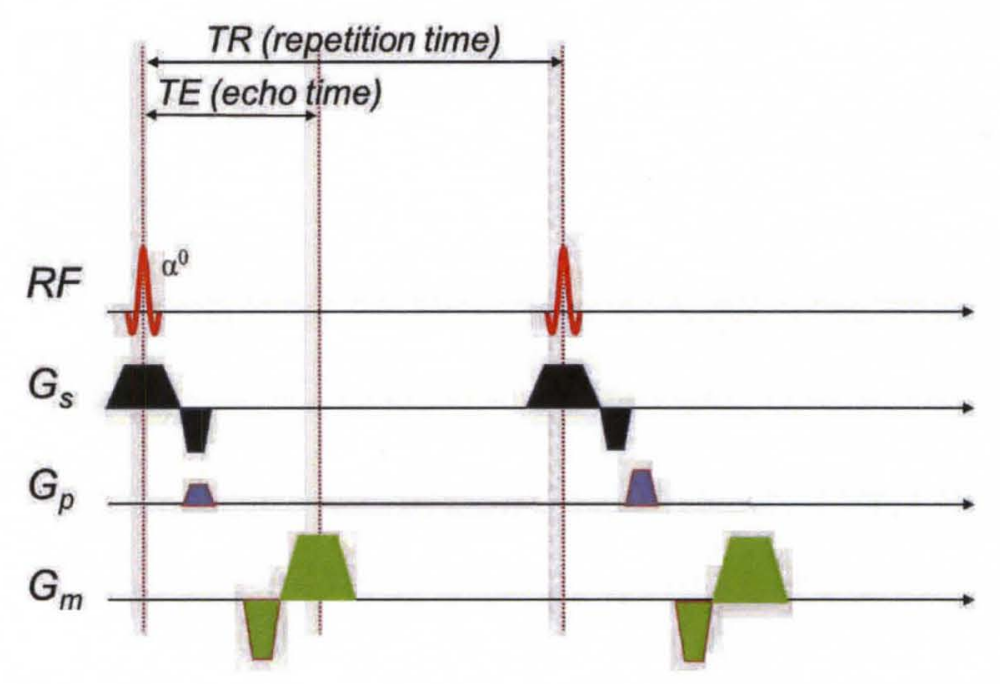

FIGURE 11 -Diagram of gradient echo sequence. $G_{s}$ is slice selection gradient. $G_{p}$ is phase encoding gradient. $G_{m}$ is frequency encoding gradient. [14]

acquisition is now used to acquire k-space lines in other slices.

A 3D volume MR acquisition simultaneously excites an entire set of contiguous slices at each $T R$ interval. The set of slices is called a chunk or a slab, and an individual element within the slab is called a partition, section, or simply a slice. The most common acquisition strategy for 3D MR imaging is to use rectilinear sampling. There are two phase encodings. The second one is called phase encoding 2, or slice encoding, to distinguish it from in-plane phase encoding. The resulting raw data fills a 3D k-space matrix, which is reconstructed by a 3D Fourier transform. The slices in the 3D slab can be displayed as individual 2D slices. The main advantage of 3D acquisitions is their ability to acquire thin contiguous slices that are ideal for volume rendering, multi-planar reformatting (MPR), or maximum intensity projection (MIP). The disadvantage of $3 \mathrm{D}$ acquisition is its prolonged scanning time compared to $2 \mathrm{D}$ acquisition. Most 3D pulse sequences use gradient echoes due to their short $T R$.

There are three common types of trajectories in k-space: cartesian (rectilinear), radial, and spiral. k-space order, view order, or phase order, is the way to arrange the order of the trajectories. For rectilinear k-space trajectory, there are three commonly 
used k-space order: sequential order, centric order, and reverse centric order. For 3D acquisition trajectory, except cartesian trajectory, another acquisition strategy is to replace in-plane phase encoding with spiral or radial trajectory. Yet another one is to completely abandon phase encoding, in stead, use 3D-projection reconstruction, in which the frequency-encoding direction varies in three dimensions. Thus, $3 \mathrm{D} \mathrm{k-}$ space is sampled by a set of radial lines. Then $3 \mathrm{D}$ images can be constructed by filtered-back-projection. 


\section{CHAPTER III MR TECHNIQUE FOR IMAGING CARDIAC FUNCTION}

MRI has revolutionized medicine due to its broad ability to image many organ systems. However, in the case of the heart, development of MRI has been hampered because of heart's inherent motion. There are many techniques available nowadays for cardiac imaging which provide qualitative and quantitative measurements of cardiac function. In the realm of MRI, cardiac cine-MRI is considered as the standard technique for measuring global function parameters [20]. It also can reveal important regional function parameters, such as wall thickness. MR tagging $[27,28]$ is a wellknown method to track local deformations. Other techniques, such as phase contrast MRI, DENSE, and SENC, can also reveal regional properties of the heart. Several review papers have previously been published in the field of deformation recovery from cardiac MR imaging $[20,29,30]$. Castillo et al. [20] briefly reviewed MR imaging techniques in regional myocardial function analysis. Axel et al. [29] authored a review about tagged MR imaging of the heart, in which tagging methods, imaging methods for tagging as well as some tagged image analysis methods were reviewed. Epstein [30] reviewed cardiac MR methods for imaging LV function and their clinical applications to coronary artery disease.

In this chapter, MRI methods and approaches designed for imaging cardiac function will be discussed. The organization of this chapter is as follows. Section III.A briefly introduces cardiac gating/triggering. Section III.B explains cardiac cine MRI techniques. Section III.C gives an overview of two commonly used MRI tagging techniques. Section III.D summaries phase contrast MRI principles and related motion analysis methods. Section III.E and Section III.F introduce two more recent 
techniques: DENSE and SENC. The contents in this chapter are largely based on a book chapter that the author has published in [31].

\section{A. Cardiac Gating/Triggering}

The fundamental challenges to cardiac MRI are movement of the heart throughout the cardiac cycle and movement of the lungs during the respiratory cycle, both requiring mitigation to avoid motion artifacts. Respiratory motion can be alleviated with breath holding during imaging. The subject is typically asked to hold his/her breath for $10-15$ seconds which is typically sufficient to collect a cine at one slice location. It should be noted that in sick patients and/or those unable to hold their breath, navigator-gated acquisition is an alternative. Cardiac gating/triggering provides methods to synchronize data collection with the cardiac rhythm. It is used to minimize the motion artifacts arising from cardiac motion and from flowing blood or cerebrospinal fluid (CSF). The goal is to acquire an entire set of k-space data at approximately the same slice location for the cardiac cycle.

A variety of approaches to gated/triggered data acquisition can be adopted including: electrocardiography (ECG), Vectorcardiogram (VCG) and peripheral gating. However, the methods more widely used to synchronize with cardiac motion essentially rely on the ECG and VCG, since the signal from peripheral devices which provide blood oxygen saturation levels are delayed relative to the electrical activity of the heart. There are two types of triggering: prospective triggering and retrospective triggering. Prospective triggering uses the $\mathrm{R}$-wave to determine the starting point of the acquisition. Slices are acquired at the same time after each new R-wave in successive R-R intervals. Artifacts are reduced by keeping slice excitation consistent in relation to the $\mathrm{R}$-peak. In prospective triggering, only one part of the cardiac cycle is imaged. To account for heart rate variability, retrospective triggering, a continuous acquisition method, can be adopted. With retrospective triggering, $\mathrm{k}$-space data 
are continuously acquired through-out time and retrospectively "binned" relative to the $\mathrm{R}$-wave. The advantage of retrospective triggering is that it permits imaging the entire cardiac cycle, whereas in prospective gating, there is "dead" time at the end of the diastole.

\section{B. Cine MR Imaging}

Cardiac cine MRI is widely used clinically due to its high soft-tissue contrast. The temporal resolution is also high enough to do deformation analysis over the cardiac cycle. It is the gold standard for non-invasively quantifying global in vivo heart function and for measuring ejection fraction, cavity volume, and mass.

There are multiple cardiac phases that are imaged in one cardiac cycle, usually between 20 to 30 , depending on the temporal resolution (30 to 50 milliseconds per frame). Although it is possible to image the heart in real-time with cine MRI, the resulting image quality is poor. Instead, most sequences use ECG or VCG gating/triggering to acquire images at each stage of the cardiac cycle over several heart beats. This is called segmented acquisition which is the process of dividing the cardiac cycle into multiple segments (frames) to produce a series of images that can be displayed as a movie (cine). This technique is the primary basis for functional assessment by cine MRI. During one cardiac cycle, ECG-gated cine MRI acquires one segment of image raw data for each cardiac phase, as shown in Figure 12. Multiple segments comprising the complete k-space raw data are acquired in multiple heartbeats. The longer the period for each segment, the faster the acquisition, but the worse the frame rate (i.e, the fewer cardiac phases imaged). With the help of a phased-array radio frequency $(\mathrm{RF})$ receiver coil and parallel image acquisition and reconstruction techniques, typical scan times of 6 to 7 heartbeats per slice are now routine. The in-plane spatial resolution of cine MRI is on the order of $1.5 \times 2 \mathrm{~mm}^{2}$. The slice thickness is between 5 to $10 \mathrm{~mm}$ [30]. Multiple 2D imaged slices may be 


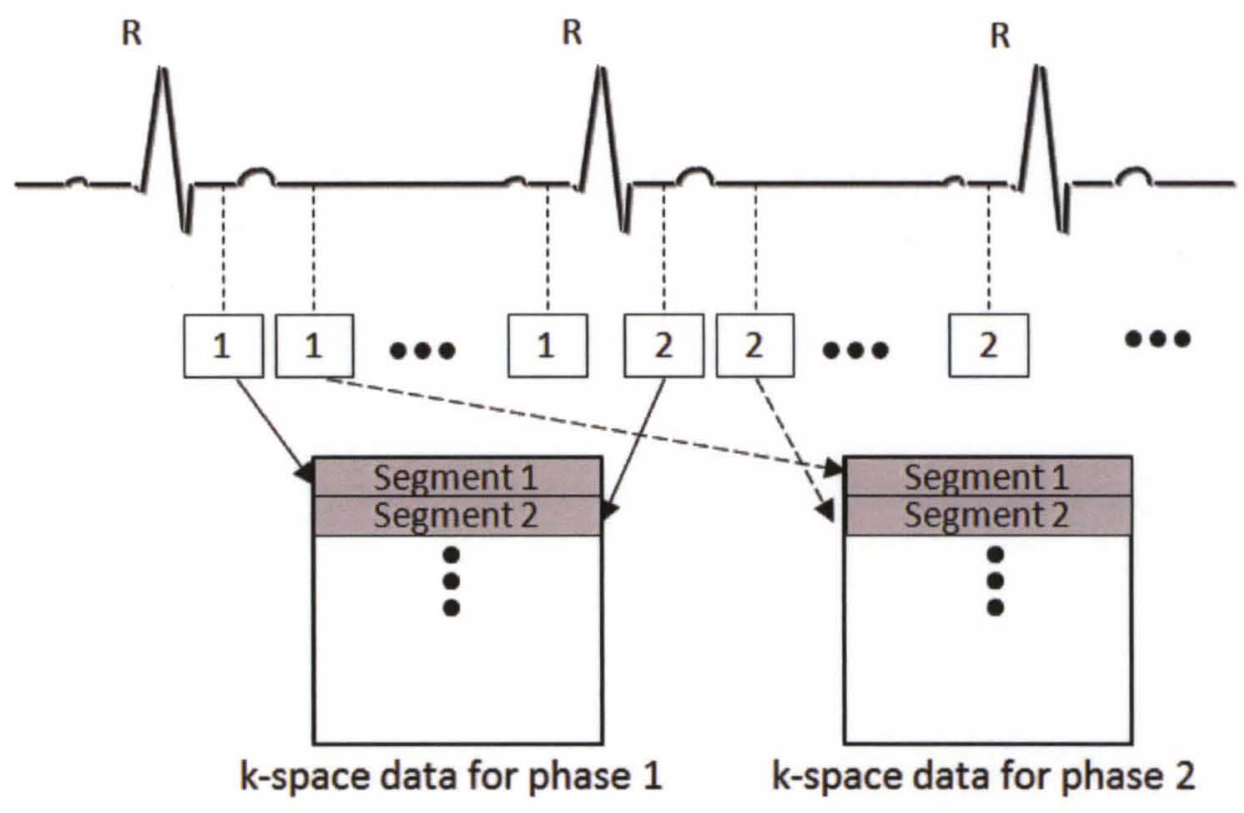

FIGURE 12-Schematic diagram of ECG-gated breath-hold cine MRI. There are multiple cardiac phases in one cardiac cycle. For each phase, a segment is acquired in one heart beat.

stacked to construct a 3D volume for each cardiac phase.

Cine gradient echo sequences, often known as bright blood sequences, are commonly used for evaluating cardiac function. Blood appears bright in these sequences due to the contrast properties of blood and its rapid flow. The technique can discriminate between blood and myocardium very well. Two imaging pulse sequences are most commonly used for acquiring cine MRI: spoiled gradient echo (GRE) and balanced steady-state free precession (b-SSFP) [14]. b-SSFP can yield better signalto-noise ratio (SNR) and contrast-to-noise ratio (CNR) than spoiled GRE and is less sensitive to motion-induced signal loss. Therefore, cine MR images using b-SSFP appear more uniform within the blood and are darker in the myocardium. b-SSFP gradient echo sequences have largely replaced spoiled gradient echo sequences for this purpose. Different trade names for these b-SSFP sequences are TrueFISP (True Fast Imaging with Steady-state Precession; Siemens), FIESTA (Fast Imaging Employing Steady-state Acquisition; GE), and b-FFE (Balanced Fast-Field Echo; Phillips). 

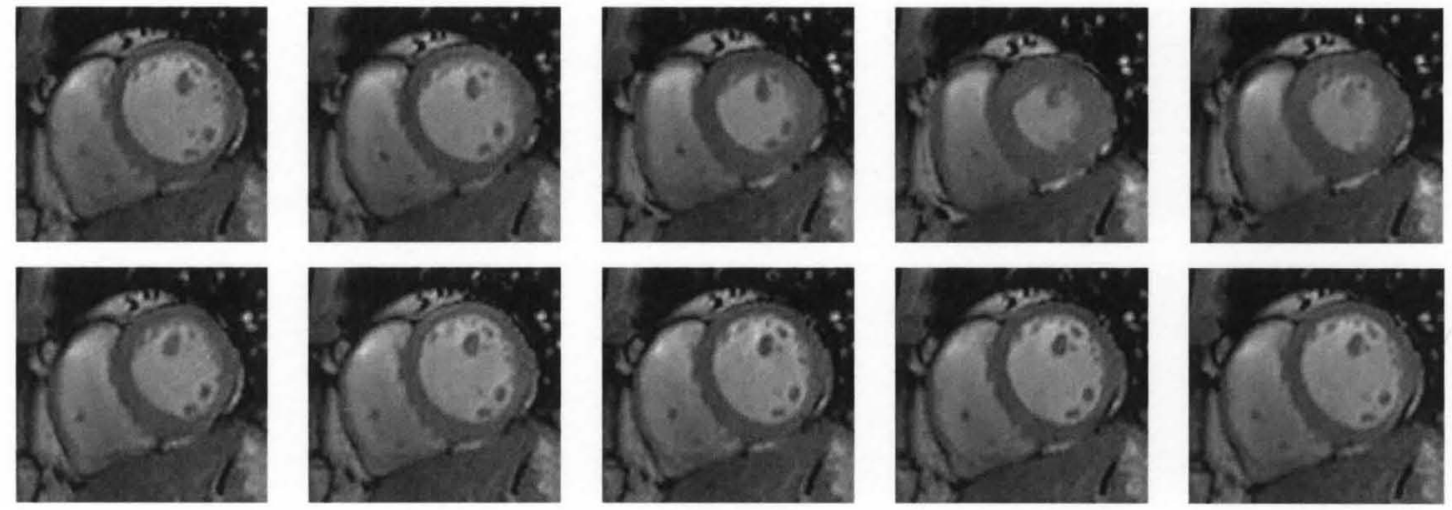

FIGURE 13-A sequence of short-axis cine images acquired with b-FFE on a Philips Achieva 3T scanner. Ten out of thirty phases are shown from the top left to bottom right. The first row shows systolic images and the second row shows the diastolic images. Imaging parameters were as follows: $\mathrm{TE}=1.603 \mathrm{~ms}$, slice thickness $=$ $8 \mathrm{~mm}$, spatial resolution $=1.25 \times 1.25 \mathrm{~mm}^{2}$, acquisition matrix $=160 \times 187$, flip angle $=45^{\circ}$. A phased-array thoracic coil with 16 elements was used.

These sequences are typically used in conjunction with segmented k-space acquisition. Segmented acquisition is the process of dividing the cardiac cycle into multiple segments (phases) to produce a series of images that can be displayed as a movie (cine). Each image in the cine is typically constructed from information gathered over several heart beats - within a breath hold of 10 to 20 seconds depending on the sequence. Figure 13 and 14 show a sequence of short-axis (SA) and long-axis (LA) cine images, respectively. Thirty frames were required using a b-FFE sequence on a Philips 3T Achieva scanner showing the contraction wave during the cardiac cycle. One third of them have been displayed here. The first row shows systolic images and the second row shows diastolic images.

Since to a large extent the myocardium appears homogeneous in cine MR images, cine MRI can not directly provide information on myocardial motion patterns, e.g., relative rotation of the endocardial layer relative to the epicardial layer (transmural shear), or of basal slices with respect to apical slices (torsion) [32]. The most commonly used features in cine MRI are endocardial and epicardial contours, 

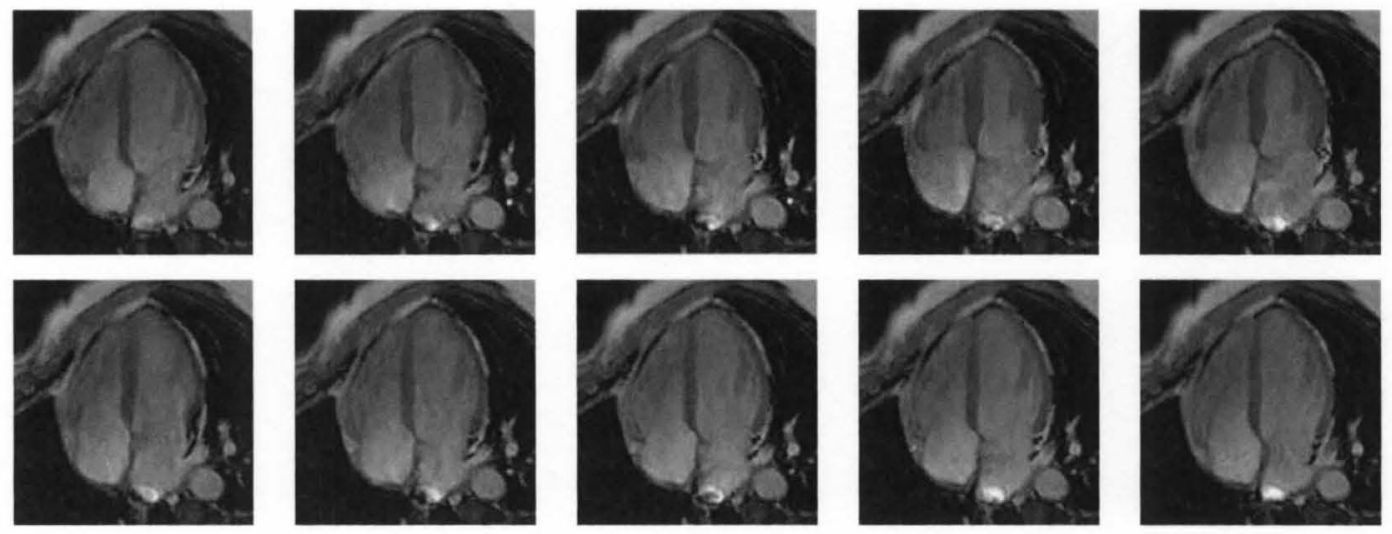

FIGURE 14-A sequence of long-axis cine images acquired with b-FFE on a Philips Achieva 3T scanner in the same volunteer whose short-axis images were shown in Figure 13. Ten out of thirty phases are shown from the top left to bottom right. The first row shows systolic images and the second row shows diastolic images. Imaging parameters were as follows: $\mathrm{TE}=1.737 \mathrm{~ms}$, slice thickness $=8 \mathrm{~mm}$, spatial resolution $=1.21 \times 1.21 \mathrm{~mm}^{2}$, acquisition matrix $=176 \times 211$, flip angle $=45^{\circ}$. A phased-array thoracic coil with 16 elements was used.

or specific feature points on these contours. For motion analysis from cine MRI, a useful model that could be utilized is its incompressibility. One strategy for motion tracking may be to first track sparse feature points on the contours and subsequently perform dense motion field reconstruction. Reconstruction of the dense motion field from a sparse set of landmarks is an ill-posed problem and therefore additional constraints are needed to obtain a unique solution. The constraints previously proposed in the literature are either mathematical or mechanical, enforcing smoothness of the deformation field subject to additional requirements [33-35]. The other strategy is to use image registration [36-39], either for the whole image or for some feature points. Due to the substantial challenges in deformation recovery from cine MRI including significant modeling needs, it is reasonable to assume that the accuracy of cine MRI deformation recovery techniques are not on par with the methods that will be described later in this chapter.

\section{MRI Tagging}




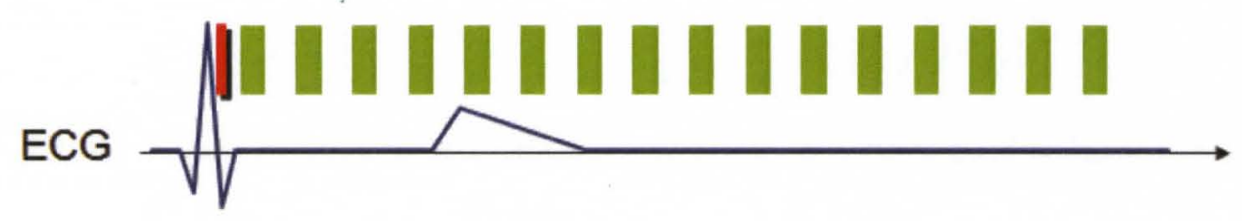

Tagging

\section{Multiple Heart Phase Imaging}

FIGURE 15 - Tagging sequence timing scheme. Tagging pulse is usually applied at end-diastole, immediately after the R-wave.

Myocardial tagging is a technique that is useful in assessing ventricular function. Myocardial tagging was first introduced by Zerhouni et al. [27] and Axel et al. [28] in 1988 and 1989. The process of MR tagging uses a special pulse sequence to spatially modulate the longitudinal magnetization in the imaging volume, prior to image acquisition using conventional imaging, as shown in Figure 15. The tagging sequence is usually applied immediately after the R-peak. The varying magnetization produces alternating light and dark patterns on the image as noninvasive markers in the tissues. The main reason why tagged MRI can image motion is that when the local magnetization of a material point is altered, the material point maintains the altered magnetization when it moves within the limits of the $T_{1}$ relaxation time.

Spatial Modulation of Magnetization (SPAMM) [28] is the most commonly used technique to produce sinusoidal tag patterns. Optimal tagging and acquisition of MR images for cardiac motion analysis was investigated by Nguyen et al. [40]. Pai and Axel [41] gave a thorough review of tagged cardiac MR imaging methods, including advances in pulse sequence development, image acquisition, high temporal and spatial resolution imaging, high field strength imaging, and 3D whole heart tagging. A review paper covering the current clinical applications of myocardial tagging is [42]. 


\section{Spatial Modulation of Magnetization (SPAMM)}

A one-dimensional 1-1 SPAMM sequence is shown in Figure 16 (Left) which consists of two $90^{\circ} \mathrm{RF}$ pulses, an interspersed tagging gradient in the readout direction, and a spoiler gradient. Before the first RF pulse, magnetization in rotating frame is initially all polarized along the main magnetic field (in the $\mathrm{z}$ direction) (Figure 16 (a)). The first RF pulse flips the initial longitudinal magnetization into the transverse plane (Figure $16(\mathrm{~b})$ ). The tagging gradient $G_{t}$ produces a periodic spatial modulation of the phase of the transverse magnetization along the gradient direction (Figure 16 (c)). The second RF pulse produces modulated longitudinal magnetization (Figure 16 (d)). The function of the spoiler gradient is to eliminate any remaining transverse magnetization. A tag grid can be produced by following the second RF pulse with a second gradient in orthogonal direction to the first gradient and then with another RF pulse. Some tagged and non-tagged images for a dog at corresponding slice locations are shown in Figure 17. In the subsequent imaging step, the spatially modulated longitudinal magnetization is made visible by the excitation RF pulse which flips it to the transverse plane. The tag lines are not sharply changed, but with sinusoidal variation of intensity in the image (Figure 16 (d)). An MR tagged image can be considered to be product of two components. The first component is signal from the anatomy, and the second is the sinusoidal tagging component. Therefore, tagged image produced by sequence in Figure 16 amounts to amplitude modulation (AM) of the anatomical image by a carrier frequency prescribed by tagging pulse. Sharper tagging stripes can be obtained by using other binomially distributed RF pulses that are each separated by dephasing gradients [43]. The effect of the SPAMM pulse sequence is to produce a series of stripes in the acquired images. One drawback of SPAMM tagging is that due to $T_{1}$ relaxation, tags fade in later phases of the cardiac cycle. 

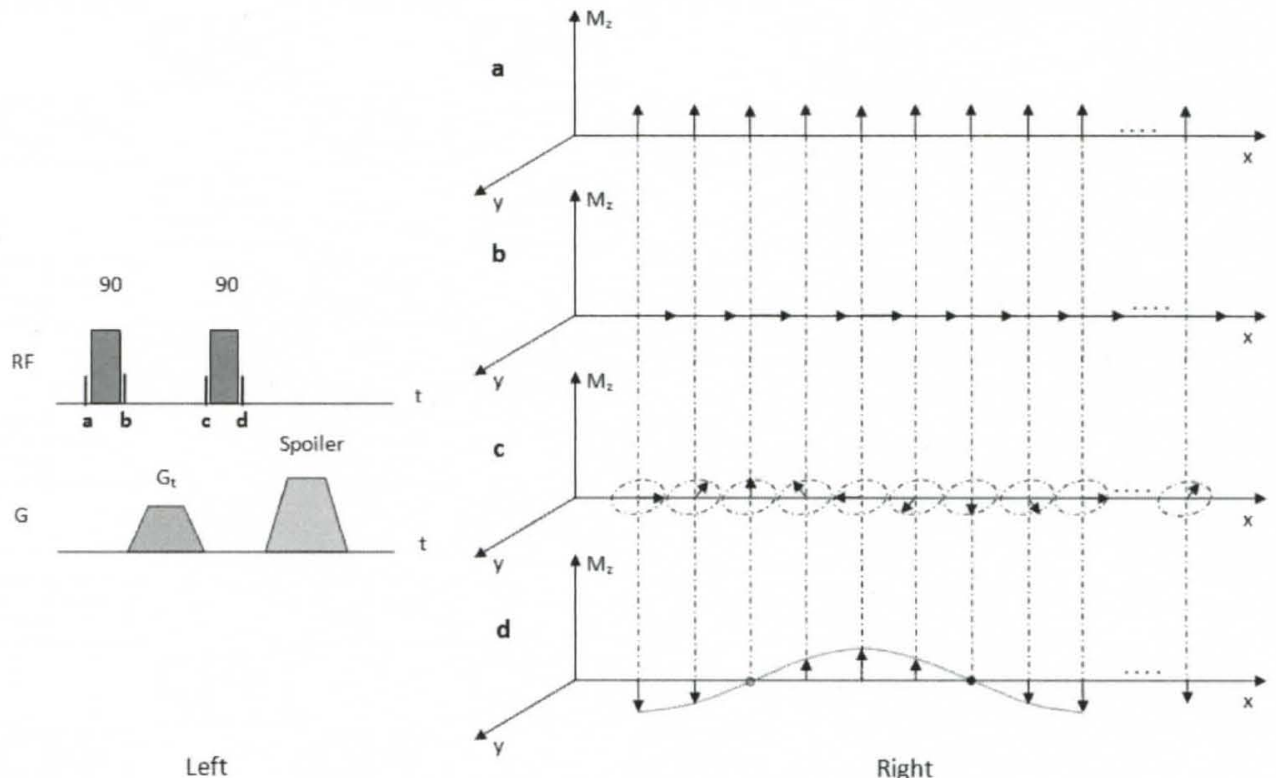

FIGURE 16-SPAMM pulse sequence. Left: Timing diagram for SPAMM. RF = radio-frequency excitation, $\mathrm{t}=$ time, $\mathrm{G}_{t}=$ wrap gradient for production of modulation. Letters a-d indicate corresponding time points in the right figure. Right: State of magnetization as a function of time. (a) Magnetization prior to initiation of the modulation sequence. (b) Magnetization after first RF pulse. (c) Magnetization after wrap (modulating) gradient pulse. (d) Longitudinal magnetization after second RF pulse.
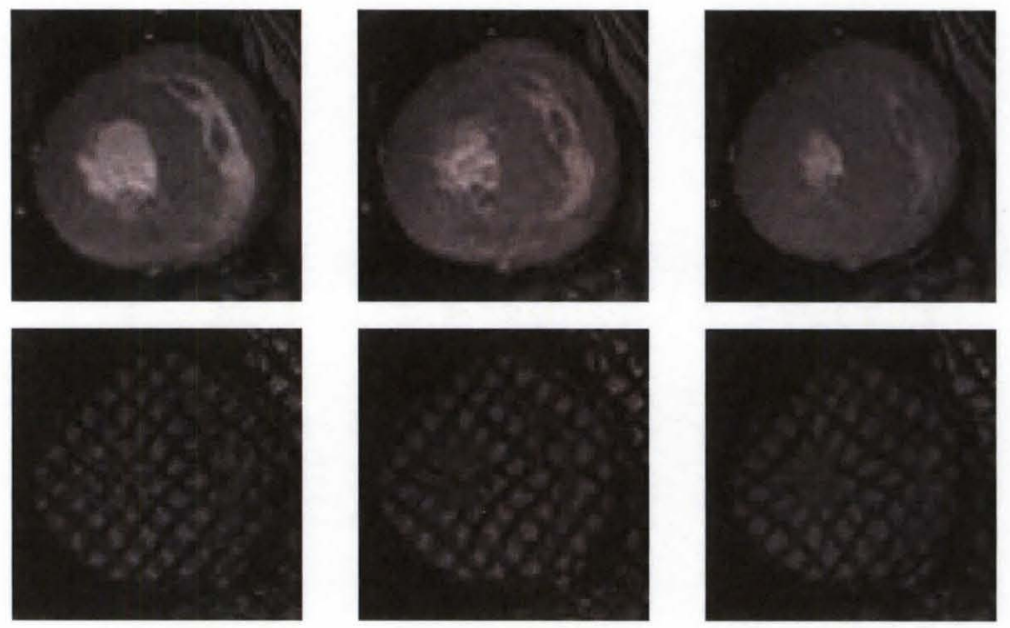

(a)

(b)

(c)

FIGURE 17 - The top row shows a sequence of short-axis mid-ventricular untagged images taken from a dog at three different time points in the cardiac cycle. (a) enddiastole, (b) mid-systole, and (c) end-systole. The bottom row shows tagged images at corresponding times and locations. 


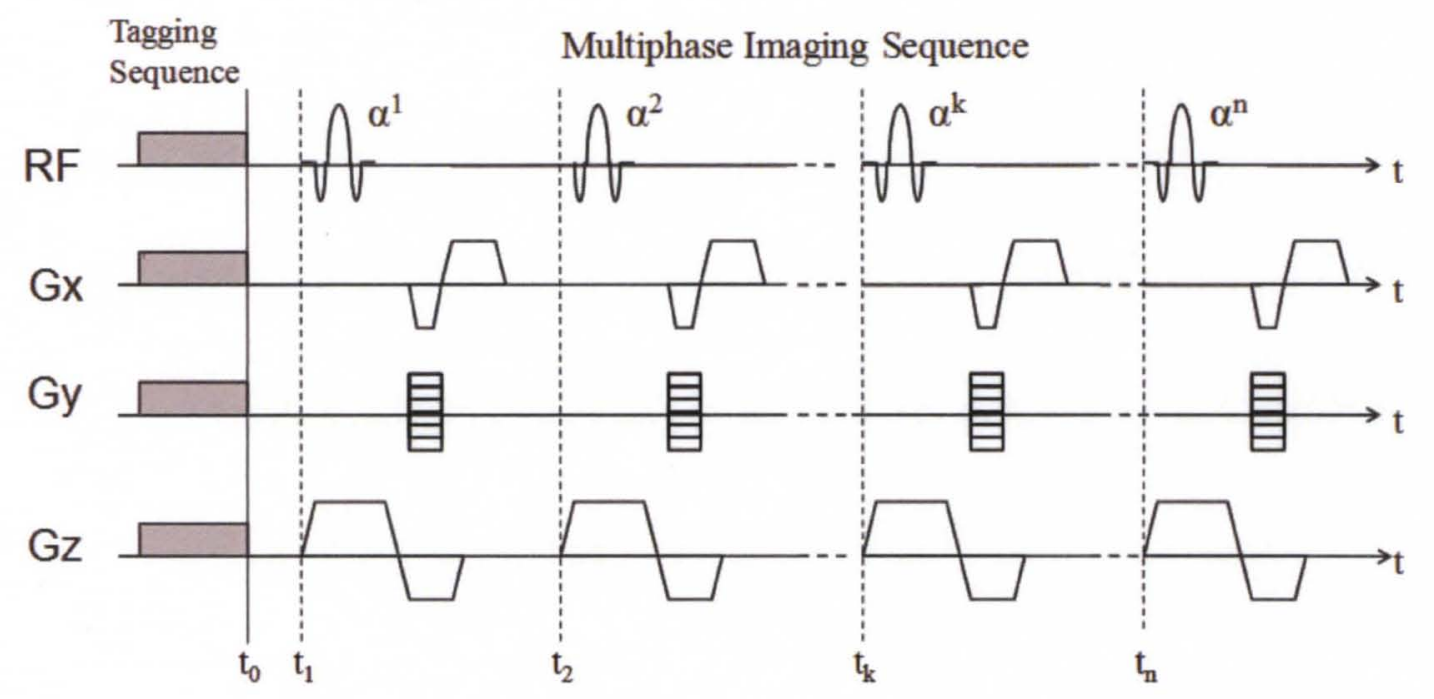

FIGURE 18 - Timing diagram of a typical tagging experiment. A tagging sequence is applied before $t_{0}$ followed by a standard multiphase imaging sequence. $t_{k}$ corresponds to the start of the $k^{\text {th }}$ phase in the cardiac cycle. $\alpha^{k}$ corresponds to the RF pulse flip angle at $k^{\text {th }}$ phase in the cardiac cycle where $\alpha^{1}=\cdots \alpha^{k}=\cdots \alpha^{n}$. Note that each of the RF pulses $\alpha^{1}, \alpha^{2}, \cdots$ is applied multiple times corresponding to different strength of the phase encoding gradient. That creates a single image in the cine sequence.

2. Complementary Spatial Modulation of Magnetization (CSPAMM)

Complementary SPAMM (CSPAMM) was introduced by Fischer et al. to improve tagging contrast in later phases of the cardiac cycle [44]. Two SPAMM images which are out of phase by $180^{\circ}$ are acquired and subtracted. CSPAMM has the advantage of longer net tag persistence while suppressing untagged blood. In CSPAMM, the longitudinal magnetization $M_{z}$ is decomposed into two terms: one for tagging information $Q_{T}$ and the other for the relaxation part $Q_{R}$ (explicit signal expressions will be given for these shortly). A timing diagram of a typical tagging experiment is shown in Figure 18. At time $t_{0}$ immediately after the SPAMM tagging sequence, the modulated longitudinal magnetization is:

$$
M_{z}\left(t_{0}\right)=M_{s s} T A G(x, y)
$$


where $M_{s s}$ is the steady state magnetization before tagging and $T A G(x, y)$ represents a spatially varying sinusoidal function introduced by tagging sequence. At time $t_{1}>$ $t_{0}$, longitudinal magnetization becomes

$$
\begin{aligned}
M_{z}\left(t_{1}\right) & =\left(M_{z}\left(t_{0}\right)-M_{0}\right) e^{-t_{1} / T_{1}}+M_{0} \\
& =\left(M_{s s} T A G(x, y)-M_{0}\right) e^{-t_{1} / T_{1}}+M_{0} \\
& =\underline{\left.M_{s s} T A G(x, y)\right) e^{-t_{1} / T_{1}}}+\underline{M_{0}\left(1-e^{-t_{1} / T_{1}}\right)} \\
& =Q_{T_{1}}+Q_{R_{1}}
\end{aligned}
$$

where $M_{0}$ is the equilibrium magnetization, $T_{1}$ is the longitudinal relaxation time. At time $t_{k}$,

$$
\begin{aligned}
M_{z}\left(t_{k}\right) & =\left(M_{z}\left(t_{k-1}\right)-M_{0}\right) e^{-\left(t_{k}-t_{k-1}\right) / T_{1}}+M_{0} \\
& =\left[\left(Q_{T_{k-1}}+Q_{R_{k-1}}\right) \cos \alpha_{k-1}-M_{0}\right] e^{-\left(t_{k}-t_{k-1}\right) / T_{1}}+M_{0} \\
& =\left(Q_{T_{k-1}} \cos \alpha_{k-1}+Q_{R_{k-1}} \cos \alpha_{k-1}-M_{0}\right) e^{-\left(t_{k}-t_{k-1}\right) / T_{1}}+M_{0} \\
& =Q_{T_{k-1}} \cos \alpha_{k-1} e^{-\left(t_{k}-t_{k-1}\right) / T_{1}}+\left(Q_{R_{k-1}} \cos \alpha_{k-1}-M_{0}\right) e^{-\left(t_{k}-t_{k-1}\right) / T_{1}}+M_{0} \\
& =Q_{T_{k}}+Q_{R_{k}}
\end{aligned}
$$

Therefore, the two components of the longitudinal magnetization just before the $k_{t h}$ RF pulse are:

$$
\begin{aligned}
Q_{T_{k}} & =Q_{T_{k-1}} \cos \alpha_{k-1} e^{-\left(t_{k}-t_{k-1}\right) / T_{1}} \\
& =\left(Q_{T_{k-2}} \cos \alpha_{k-2} e^{-\left(t_{k-1}-t_{k-2}\right) / T_{1}}\right) \cos \alpha_{k-1} e^{-\left(t_{k}-t_{k-1}\right) / T_{1}} \\
& =\ldots \\
& =M_{s s} T A G(x, y) e^{-t_{k} / T_{1}} \prod_{j=0}^{k-1} \cos \alpha_{j} \\
Q_{R_{k}} & =\left(Q_{R_{k-1}} \cos \alpha_{k-1}-M_{0}\right) e^{-\left(t_{k}-t_{k-1}\right) / T_{1}}+M_{0}
\end{aligned}
$$

where $Q_{T_{k}}$ is the tagging component, while $Q_{R_{k}}$ is the relaxed term. After the $k_{t h}$ RF imaging pulse of flip angle $\alpha_{k}$, the longitudinal magnetization is rotated to the 


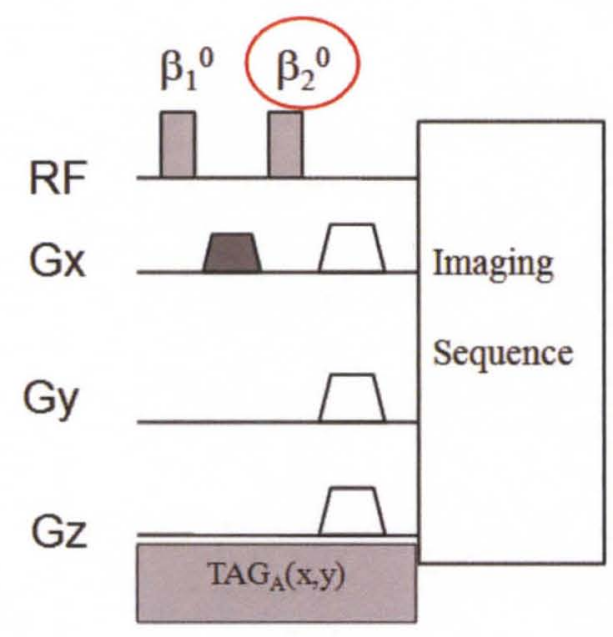

(a)

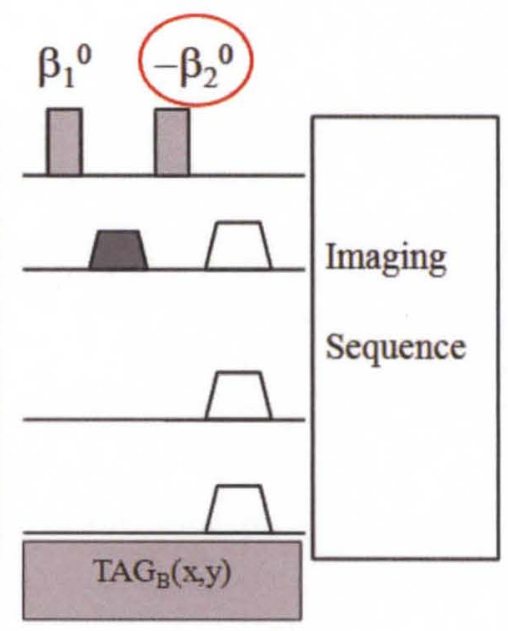

(b)

FIGURE 19-Timing diagram of a 1-1 CSPAMM sequence. (a) Measurement with positive tagging pattern $T A G_{A}(x, y)$ (b) Measurement with negative tagging pattern $T A G_{B}(x, y)$

transverse plane which contributes to the $k_{t h}$ image.

$$
I_{k}=M_{z}\left(t_{k}\right) \sin \alpha_{k} e^{-T E / T_{2}^{*}}=\left(Q_{T_{k}}+Q_{R_{k}}\right) \sin \alpha_{k} e^{-T E / T_{2}^{*}}
$$

The basic idea of CSPAMM is to eliminate the relaxation term $Q_{R_{k}}$ while only keeping the tagging information term $Q_{T_{k}}$ by acquiring two images $A_{k}$ and $B_{k}$ using the same parameters except for their respective tagging patterns $T A G_{A}(x, y)$ and $T A G_{B}(x, y)$ (See Figure 19). The subtraction of both $k_{t h}$ images leads to

$$
A_{k}-B_{k}=M_{s s}\left[T A G_{A}(x, y)-T A G_{B}(x, y)\right] e^{-t_{k} / T_{1}}\left(\prod_{j=0}^{k-1} \cos \alpha_{j}\right) \sin \alpha_{k} e^{-T E / T_{2}^{*}}
$$

Visualization of magnetization subtraction in CSPAMM is shown in Figure 20. The positive sinusoidal SPAMM tagging pattern is shown in solid lines and the negative sinusoidal SPAMM tagging pattern is shown in dashed line. 'The subtracted CSPAMM pattern is shown in dotted line. Visualization of k-space for an image modulated by a cosine tagging function in the horizontal direction is shown in Figure 21 and for the vertical direction is shown in Figure 22. In both figures, (a) shows the k-space for the SPAMM with positive tagging pattern, (b) shows the k-space for the 

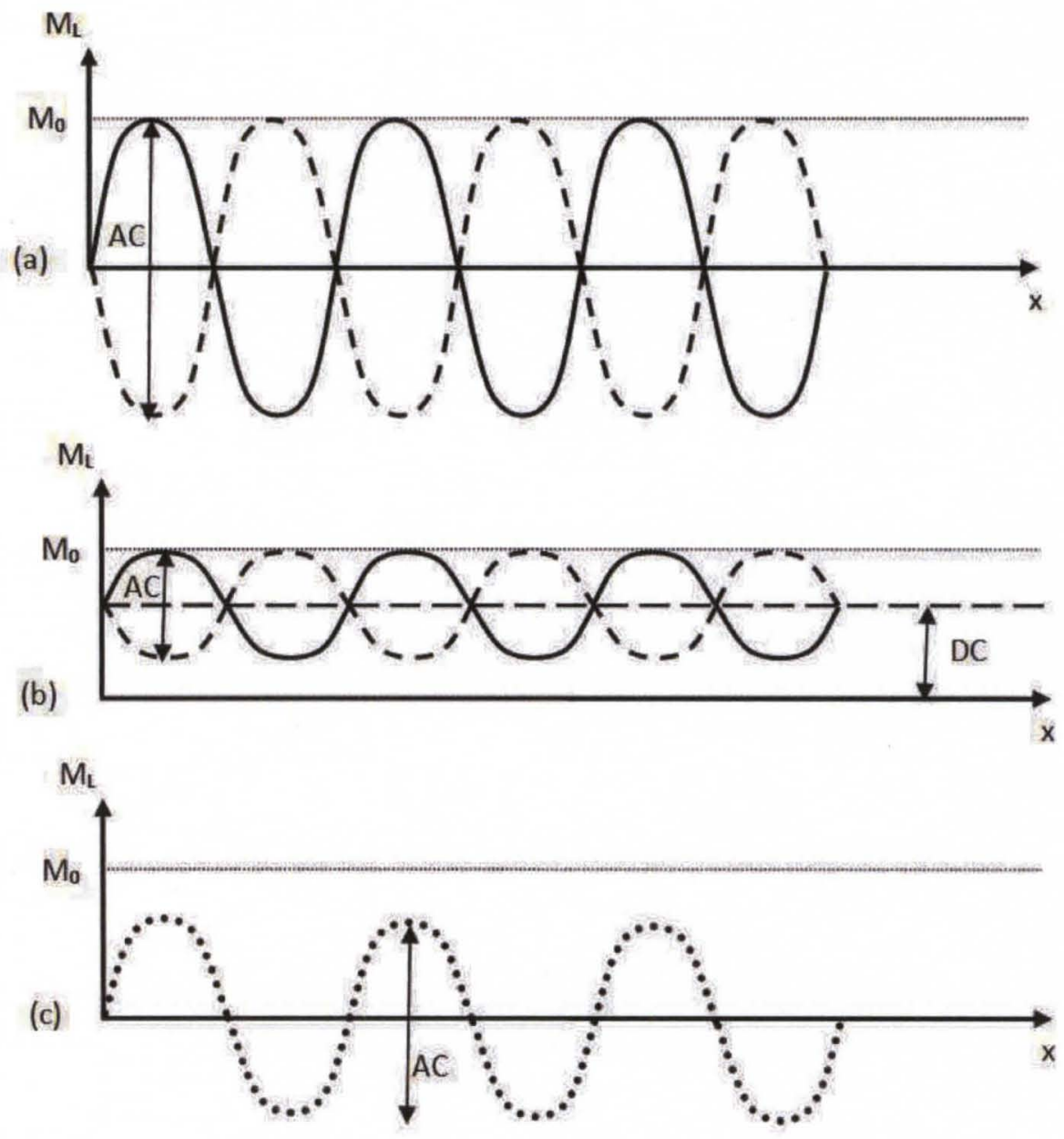

FIGURE 20-Visualization of magnetization subtraction in CSPAMM. The positive sinusoidal SPAMM tagging pattern is shown with solid lines and the negative sinusoidal SPAMM tagging pattern is shown with dashed lines. (a) Shows the magnetization in the initial state immediately after tagging pulse sequence. With time, longitudinal relaxation occurs. (b) Shows the magnetization after a certain time period. (c) Shows the CSPAMM tagging pattern which results from the subtraction of the negative pattern from the positive pattern in (b). 


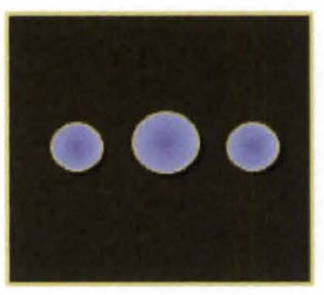

(a)

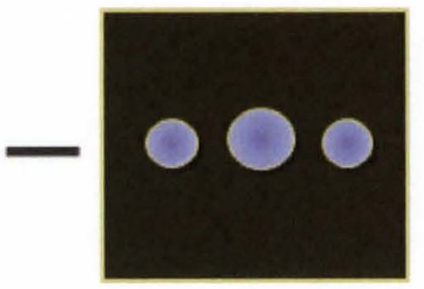

(b)

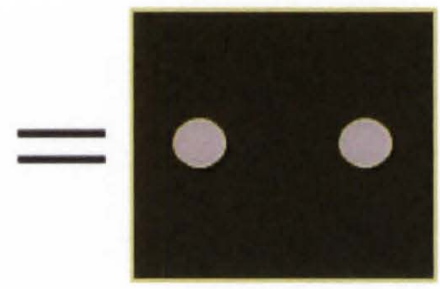

(c)

FIGURE 21 - Visualization of k-space for an image modulated by a cosine in the horizontal direction. (a)k-space for one SPAMM with positive tagging pattern (b)kspace for the other SPAMM with negative tagging pattern (c)k-space for CSPAMM.

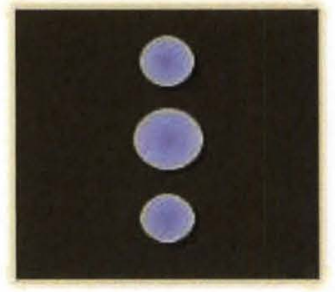

(a)

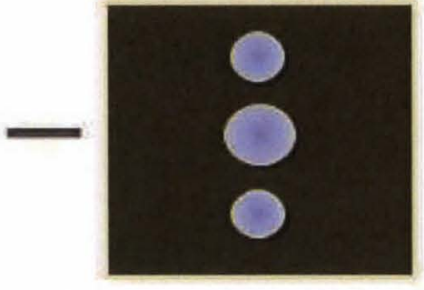

(b)

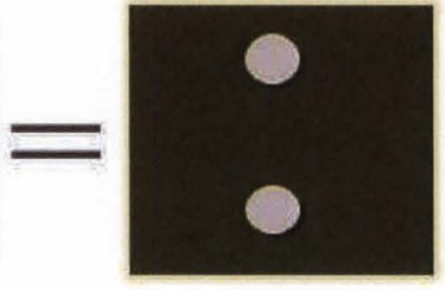

(c)

FIGURE 22-Visualization of $\mathrm{k}$-space for an image modulated by a cosine in the vertical direction. (a)k-space for one SPAMM with positive tagging pattern (b)kspace for the other SPAMM with negative tagging pattern (c)k-space for CSPAMM.

other SPAMM with negative tagging pattern, and (c) is the k-space for CSPAMM which is the subtraction of (b) from (a).

\section{Phase-Contrast MRI}

Phase contrast MR imaging (PCMRI) is also called velocity-encoded MRI [45]. Phase contrast methods are based on the fact that moving spins accumulate a different phase offset than static spins in the presence of a magnetic field gradient. The phase shift is proportional to the product of tissue velocity and the first moment of the magnetic field gradient in the direction of motion (See Equation (46)). PCMRI sequence uses a bipolar gradient as shown in Figure 23 which is first positive at a particular amplitude and duration and then negative for an equal amplitude and 


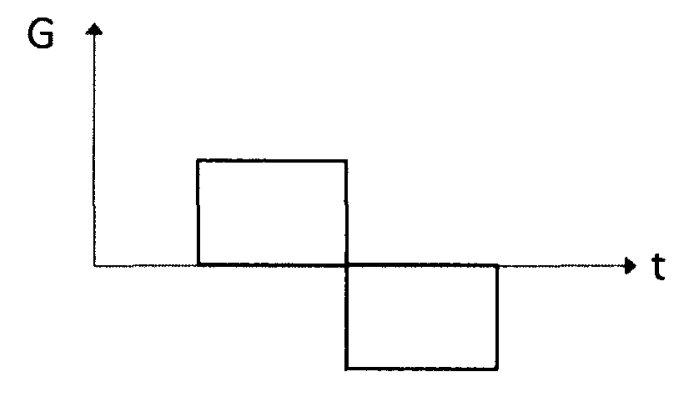

FIGURE 23-A bipolar gradient lobe.

duration to encode the velocity. For static spins, the accumulated phase under the bipolar gradient is zero. However, for spins that move at a constant velocity, there is an accumulated phase shift. The accumulated phase at time TE is:

$$
\Phi=\gamma \int_{0}^{T E} G(t) r(t) d t
$$

where $G(t)$ is the gradient, $r(t)$ is the position in the direction of $G(t)$, and $\gamma$ is the gyromagnetic ratio. The Taylor expansion of position vector $r(t)$ on initial position $r_{0}$ is:

$$
r(t)=r_{0}+v t+a \frac{t^{2}}{2}+\cdots
$$

Under the condition that there are no phase shifts from initial position $r_{0}$ (which is the case for bipolar gradient) and the phase shifts due to higher order terms are small, Equation (44) becomes:

$$
\Phi=v\left(\gamma M_{1}\right)
$$

where $M_{1}$ is the first moment of the gradient waveform evaluated at TE.

$$
M_{1}=\int_{0}^{T E} G(t) t d t
$$

This forms the foundation for PCMRI. In deriving this expression, constant velocity during the time $(0, T E)$ has been assumed.

Unfortunately, the phase can be affected by many other factors, including magnetic field inhomogeneity, tissue magnetic susceptibility, pulse sequence tuning, 
and eddy currents due to gradient switching. Therefore, to accurately extract the motion of interest, phase shift due to these sources have to be eliminated. In order to do that, the procedure in PCMRI consists of a reference scan without velocity encoding, followed by two or three further acquisitions with velocity encoding along different directions $[45,46]$. The reference scan is used to correct undesired phase offsets of the second acquisition with velocity encoding. Since the phase of each pixel is modulated by velocity, one can obtain functional data with maximum resolution, the same spatial and temporal resolution as the underlying anatomical images. Velocity information in PCMRI can be used directly to calculate strain rate. The motion of the myocardium (i.e., displacements) may be found by integration with respect to time. Gradient system performance is critical in accurately extracting velocity information with phase contrast methods.

Reese et. al. [47] developed a single-shot 3D PCMRI data acquisition sequence by acquiring two contiguous $2 \mathrm{D}$ images in k-space in a single echo train of an EPI readout. Markl et al. [48] developed a fast 2D PCMRI protocol based on a black blood k-space segmented gradient echo imaging. The in-plane velocity information can be obtained within a single breath-hold measurement by choosing optimal number of k-space lines and view-sharing technique. Jung et al. [49] proposed a high-temporalresolution phase contrast MRI acquisition method using a prospective respiratory gating technique with two navigators in each cardiac cycle. The usage of dual navigator gating and view sharing [50] increased the temporal resolution to $13.8 \mathrm{~ms}$. This method was applied to investigate details in LV motion patterns [51,52]. A schematic visualization model and correlation analysis on myocardial velocities in different segments and slices were done to a large number of 58 normal subjects in three different age groups and one patient with dilated cardiomyopathy [53].

\section{E. Displacement Encoding with Stimulated Echoes (DENSE)}


DENSE is a quantitative imaging technique that encodes tissue displacement in the phase of the acquired signal. In order to overcome the low resolution limit of tagged MRI and bypass the integration required in PCMRI in order to obtain displacements, Aletras et al. [15] were the first to apply stimulated echo techniques for quantification of myocardial strain, named Displacement Encoding with Stimulated Echoes (DENSE), which combines many of the advantages of both tagging and phase velocity mapping. DENSE is a high-resolution myocardial displacement mapping method. Similar to PCMRI, it uses a bipolar gradient to encode motion of spins into the phase of the MRI signal. Figure 24 shows a timing diagram of DENSE in readout direction. Using stimulated echo, the phase is modulated and demodulated by the gradients applied between the first two RF pulses $G_{1}$ and the one after the third RF pulse $G_{2}$. The displacements occurring during the mix time $T M$ (time between the second and third RF pulses) is recorded in the phase of stimulated echo image. Similar to PCMRI, in order to eliminate the phase contributions from other sources, the sequence is repeated once more with a different gradient (shown in red dots in Figure 24) and subtracted from the first acquisition. In other words, the phase of DENSE images is proportional to the displacement, therefore strain can be obtained by computing the derivatives of the phase. This technique encodes motion over long time intervals. The encoded displacements are large; therefore, improved phase contrast is obtained with moderate gradient strength. For more details of this technique, refer to [15].

DENSE has also been used in detecting small focal regions of abnormal contraction in patients, in characterizing mouse ischemia models, and in tissue tracking of human hearts via 2D breath-hold imaging. In [54], DENSE was combined with a segmented echo-planar imaging readout (fast-DENSE) and applied to normal volunteers. In order to improve SNR and reduce breath-hold time, mixed echo train acquisition DENSE (meta-DENSE) was proposed in [55]. Aletras et al. [56] accelerated the image 


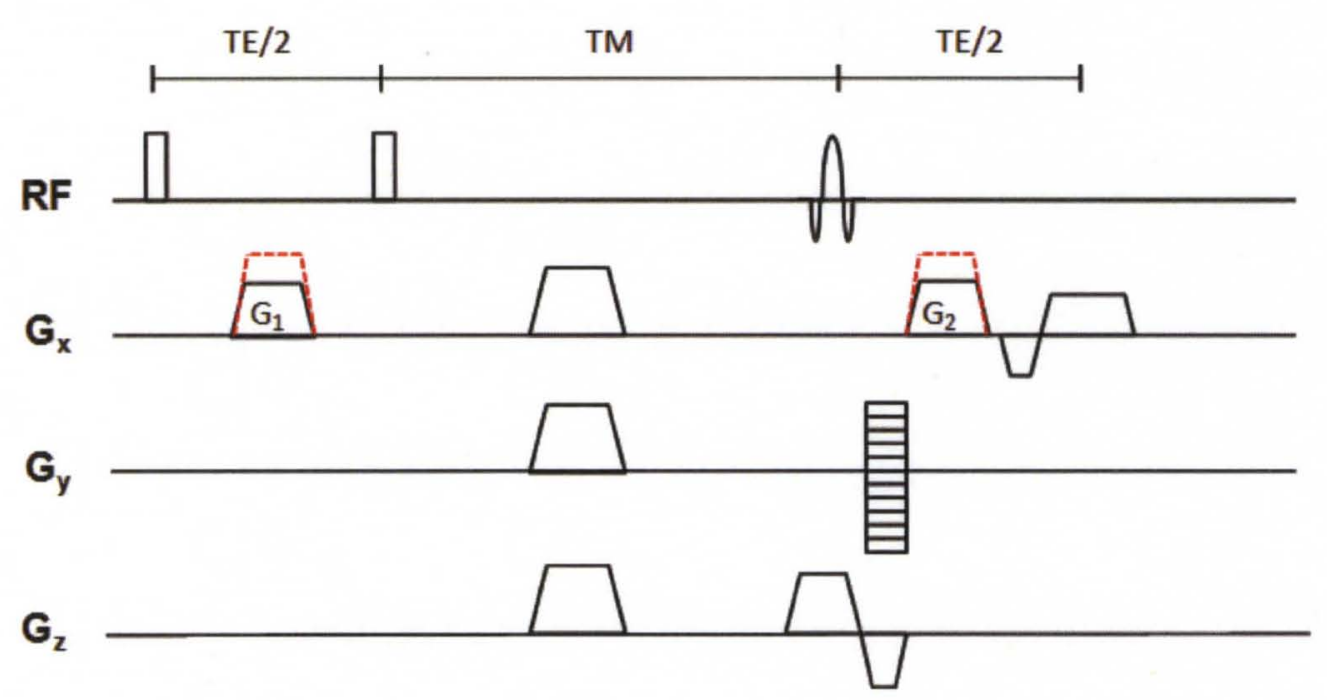

FIGURE 24-Timing diagram for DENSE in readout direction. Phase wrapping happens through the application of $G_{1} . G_{2}$ unwraps the phase [15].

acquisition process by incorporating SENSE into DENSE. This method can reduce the breath-hold time by a factor of two.

Initial DENSE sequence in [15] imaged the heart at a single cardiac phase, Kim et al. [57] developed a cine DENSE imaging sequence to sample 2D DENSE images at multiple phases of the cardiac cycle. A SPAMM sequence was used to encode the magnetization at end-diastole, then the displacement was presented as the phase shift between position encoding and readout. Cine DENSE data provide a way to track every material point in myocardium through time in the cardiac cycle. In order to improve the SNR for cine DENSE [57], a signal-averaged DENSE (sav-DENSE) was proposed [58], which extracted a pair of DENSE images with uncorrelated noise from CSPAMM image, and combined them during image reconstruction. In order to decrease the phase shifts from sources other than the encoded displacement, a general n-dimensional balanced multipoint encoding strategy was used in DENSE [59]. Spatiotemporal phase unwrapping methods were used to process cine DENSE images in [60]. In [61], slice following was incorporated into cine DENSE to track 3D cardiac motion. An adaptive phase-unwrapping and spatial filtering techniques were devel- 
oped to process cine DENSE data and compared with conventional phase-unwrapping and spatial filtering techniques [62]. The novelty of this technique is that it incorporates the location of the myocardial wall into the quality map.

Since DENSE is based on stimulated echo, there is an inherent $50 \%$ signal loss. Although DENSE and HARP may seem to be quite different at first glance, these two techniques turn out to be different implementations of phase displacement encoding. A thorough comparison of these two techniques was given in [63].

\section{F. Strain Encoding (SENC) MRI}

Strain-encoding, or SENC, is a relatively new MRI technique that can measure regional contraction, or relaxation, of the heart's myocardium. SENC pulse sequence was first developed by Osman et al. [16] to image longitudinal strain from short-axis images. SENC is built on the concept of MR tagging, but it uses tag planes parallel to the image plane. The basic concept of the SENC pulse sequence is shown in Figure 25. Strain is calculated from two images with different frequency modulation in the slice-select direction. Longitudinal strain can be computed by measuring the local tag frequency components from SA images, while circumferential strain can be measured from the LA images. The unique advantage of SENC is that it directly encodes the regional strain of the heart into the acquired image without measuring the displacement or velocity first. Using SENC imaging, the existence of stiff masses can be detected in the acquired MR images without postprocessing [64]. Pan et al. [65] proposed a fast-SENC pulse sequence which can reduce the strain image acquisition time to one heartbeat by combining localized SENC, interleaved tuning, and spiral

imaging. Youssef et al. [66] used SENC to estimate the circumferential strain of the RV free wall.

Ibrahim et al. combined SENC with slice-following to correct for the throughplane motion in real time. SENC imaging with and without slice-following can result 


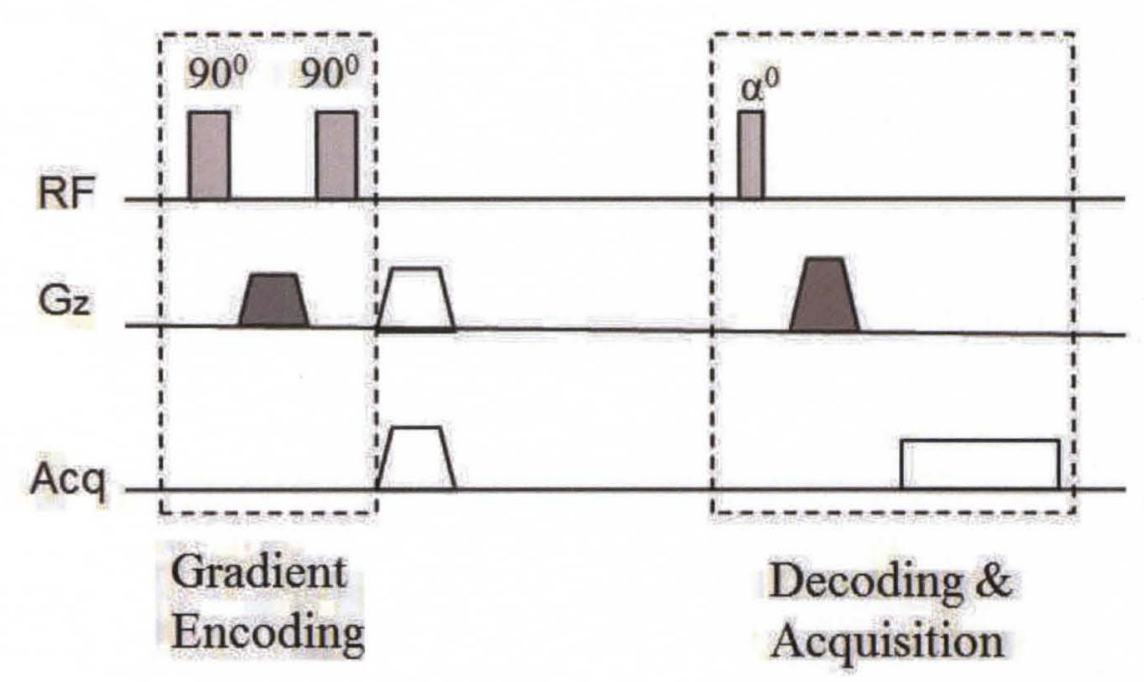

FIGURE 25-Timing diagram for SENC pulse sequence [16].

in significantly different strain values as expected with tagged MRI [67]. Ibrahim et al. [68] proposed a composite-SENC (C-SENC) pulse sequence which adjusted the tunings such that an additional image was acquired at zero frequency. Three consecutive SENC images can be obtained which contains both strain information and myocardial viability information. C-SENC produces three images: no-tuning (NT), low-tuning (LT), and high tuning (HT). Adding LT and HT images will result in an anatomical (ANAT) image of the myocardium. According to different intensity distribution in this NT-ANAT-HT 3D space, different tissue types can be classified using an unsupervised, multi-stage fuzzy clustering technique [69].

SENC is a 1D out-of-plane strain encoding method. In order to obtain 3D strain, Sampath et al. [70] integrated SENC with HARP, which can produce 2D inplane strain. In this way, 3D strains can be obtained from a single slice of SA image. Hess et al. [71] combined DENSE and SENC to obtain 3D strain map in a single slice of the myocardium.

SENC has some limitations versus MR tagging and DENSE. First, it is not capable of tracking the motion of the heart. Because it only encodes the strain 
(contractility), the exact motion of the tissue cannot be followed. Therefore, it can not be directly used to calculate quantities such as rotation and twisting. Second, SENC provides strain measurements only in the through-plane direction, and not the in-plane directions. The advantages of SENC include its simplicity, as images of strain can be directly produced in real-time. It does not require image postprocessing. Also, SENC has higher in-plane resolution of strain than MR tagging, which can be very useful in assessing regional function of the RV free wall [72]. 


\section{CHAPTER IV \\ REVIEW OF CARDIAC DEFORMATION ANALYSIS TECHNIQUES FROM MRI TAGGING}

In recent times, MRI tagging has seen increased applications and is becoming the gold standard for quantifying regional cardiac function. Local parameters such as twist, strain, and strain rate can be derived from tagged MRI data. Since its development $[27,28]$, numerous deformation analysis methods have been developed to detect tag features and track the heart motion. Several review papers have previously been published in this field $[2,73,74]$. Frangi et al. [2] gave a review of cardiac modeling techniques that were used to obtain classical cardiac functional analysis, for both global functional analysis and deformation analysis. McVeigh and Ozturk [73] summarized MRI tagging technique and image processing methods using tagged MRI for myocardial strain calculation. In 2003, Ozturk et al. [74] extended the cardiac motion tracking methods to include tagged MRI, HARP, and DENSE.

This chapter provides a survey concerning the cardiac motion analysis approaches with tagged MRI. Literature after 2000 is primarily focused; some classic methods before that will also be mentioned and summarized as needed. Different image analysis methods using tagged MRI are discussed in the subsequent sections. The contents in this chapter are based on a review paper that was published in IEEE Transactions on Medical Imaging [75].

\section{A. Tracking Myocardial Beads/Landmark-based Methods}

Myocardial beads, defined as the intersection points of orthogonal tagging planes, can be used as noninvasive markers [76]. Compared to physical implanted 
markers, tagged MRI offers a larger number of MR markers in the myocardium, with the clear advantage that they are placed noninvasively. Tracking beads is based on the fact that intersections encode a unique $3 \mathrm{D}$ position in the myocardium which move along with the deforming tissue during the cardiac cycle. Because in MRI imaging planes are fixed relative to the magnet's coordinate system throughout the cardiac cycle, as the myocardial tissue deforms, the intersections in the reference frame may move in and out of the imaging plane.

Kerwin and Prince [77] proposed a method for measuring 3D material points displacements by tracking the intersection points of a 3D grid of applied tag surfaces. In order to extract the intersections, each tag surface was reconstructed from image data using a thin-plate spline. After the tag planes were constructed, the intersection points were determined using an iterative approach, alternating projection algorithm, for the reason that each thin-spline equation may involve hundreds of logarithm terms. Only the intersections within the LV boundaries were considered. Amini et al. [76] proposed a fast method to track myocardial beads by utilizing the B-spline surface to represent tag planes in $3 \mathrm{D}$ explicitly. The parametric representation of the tag surfaces leads to an easy way to compute the position of $3 \mathrm{D}$ myocardial beads by minimizing the summation of distances between any two reconstructed tag surfaces.

Instead of extracting MR beads by reconstructing tag planes, Sampath et al. [78] proposed an automatic 3D cardiac beads tracking method. A true 3D trajectories (instead of apparent motion) of the tag beads were obtained by combining two $2 \mathrm{D}$ pathlines that were computed on both SA and LA image planes using 2D HARP tracking techniques. The slice-following CSPAMM technique was used to take through-plane motion into account. A similar idea was used by Liang et al. [79], where 3D sparse displacement field was computed by combining 2D SA and LA HARP. Subsequently, a NURBS model was used for interpolation to get a dense motion field. 


\section{B. Deformable Models}

In the deformable modeling approach, a model deforms to fit the data using energy minimization or based on classical physics-based equation of motion [80]. How to deform models and apply constraints to the deformable model vary with application. The force to drive the model deformation can originate from tag intersections, tag lines, or tag surfaces. Because of the sparsity of feature information, vector field interpolation is needed to be incorporated to obtain a dense motion field for every point within the myocardial region. Interpolation methods used to date include: mathematical regularization [81], FEM [82,83], BEM [33], superquadrics [84], B-splines [76,85], incompressibility assumptions [34], and continuum-mechanics based energy minimization [86].

Clarysse et al. [87] proposed a cosine series based model to get a mathematical expression of the reconstructed displacement field for $2 \mathrm{D}+\mathrm{t}$ series of tagged $\mathrm{MR}$ images. The coefficients of cosine model were obtained by minimizing the Euclidean distances between projection of deformed tag lines and undeformed ones.

Park et al. [84] proposed a generalized primitive deformable model driven by displacement at tag intersections as image force. Haber et al. [88] modified this framework to estimate the motion and deformation of right ventricular free wall and septum. A discretized volumetric geometry model was built using the finite elements method, after extracting biventricular boundaries using active contour techniques. The 3D motion was reconstructed by fitting a geometric model to the tag surface. Hu et al. [89] presented a statistical model to estimate in vivo material properties and strain and stress distributions in both ventricles, using the displacements calculated from tagged MRI data based on Haber's work [88]. It investigated both kinematics and material properties. Instead of assuming the fiber orientation was constant over one contract cycle, Hu et al. updated the orientation of all finite elements in each time step [90]. 
Amini et al. [91] proposed an efficient thin-plate spline warp method that warped an area in the plane such that two embedded snake grids from two tagged frames were brought into registration, interpolating a dense displacement vector field. Here an assumption was made that motion of the $L V$ is $2 \mathrm{D}$, which was roughly the case toward the apical end of the heart. A generalization of TPS, Laplacian splines, were used to determine the accuracy in estimation of cardiac motion when varying the smoothness order of the Laplacian splines [92]. A major problem for techniques relying on $2 \mathrm{D}$ short-axis data $[91,93]$ is that in general they do not take into account the through-plane motion. Extended to 3D and 4D (3D B-spline solid plus 1D B-spline interpolation over time), B-spline cubic deformable models [94,95] were introduced for analysis of cardiac motion.

Tustison [85] improved the 4D B-spline model framework in [95] by using an internal energy function and enabling inserting additional control points. Ozturk and McVeigh [96] also used the 4D B-spline model to analyze cardiac motion for both ventricles. In [97], Tustison extended the 4D B-spline models proposed in [85] and [95] to NonUniform Rational B-spline (NURBS) models. The proposed NURBS models were implemented in both polar and cylindrical coordinates and were restricted to the ventricular wall. Myocardial strains were computed using this coupled biventricular NURBS model from tagged MR images. Due to the spatial resolution of MRI tags, only sparse displacement information in both short and long axis images can be used. In order to make use of more information, Chen [98] extended Tustison's work [97] by introducing dense virtual tag lines obtained from phase-based displacement estimates. In [6], a multilevel B-spline model was used to integrate displacement information obtained from tagged images in the spatial domain with displacement information obtained from spectral peaks in the frequency domain in order to improve the accuracy of motion tracking.

Instead of B-spline models parameterizing the Cartesian space, Deng and Den- 
ney $[99,100]$ proposed a 3D B-spline model based on cylindrical coordinate system. This cylindrical B-spline model also took the LV myocardial shape into account and ensured smoothness in the circumferential, radial, and longitudinal directions.

Young [101] proposed an FEM model to reconstruct 3D myocardial motion from tagged MRI. Different from the previous FEM model [102], this method did not require extracting the ventricular boundaries or tag line locations in advance. The model deformation was driven by the distance between model stripe points and image stripe points in the direction orthogonal to the tags. Wang et al. [83] proposed a method to determine the passive diastolic mechanics. The 3D deformation field was constructed from tagged MRI data and material properties were estimated by matching the observed deformations with the FE model.

\section{Optical Flow Methods}

Optical flow is a motion tracking technique first proposed in computer vision. The fundamental assumption is that image intensity remains constant along a motion trajectory. However, in tagged MR images, the intensity constancy condition is not satisfied because of the relaxation of the magnetization of the spins throughout the cardiac cycle, which causes the intensity and contrast to change from image to image. There are different ways to deal with the variable intensity in tagged MRI. Prince and McVeigh [103] used a variable brightness optical flow (VBOF) method to overcome the intensity variation in tagged MRI by introducing a term which accounts for the variable brightness of the stripes using the MR parameters $T_{1}, T_{2}$, and initial magnetization $M_{0}$. Dougherty et al. [104] utilized a Laplacian filter to compensate for intensity and contrast loss in myocardial tags. Xu et al. proposed a 3D optical flow method to extract tissue displacements from 3D tagged images [105]. For harmonic phase images (which will be introduced later) the phase of each material point undergoing motion is preserved - the idea is based on phase-based optical flow 
proposed by Fleet and Jepson [106]. Harmonic images are produced by filtering the spectral peaks in Fourier domain and extracting the spatial phase information from the inverse Fourier transform of the filtered images. Florack et al. [107] applied a multi-scale optical flow framework using HARP images. A spatio-temporal Gaussian filter was applied as a preprocessing step. It can simultaneously capture $0 t h$ and $1 s t$ order structure of the motion field. LV rotational analysis was performed using this method [108].

\section{Registration-based Methods}

Cardiac motion tracking can also be considered as a $4 \mathrm{D}$ intramodality registration problem [109]. In general, image registration is a process to find the optimal transformation that can transform one image to the other, maximizing a similarity metric between them. The advantages of registration methods are: 1) Tag detection and extraction steps are not required; 2) They are automatic without the need for user-supervision. The disadvantages of the method involve getting stuck in local minima and potential misalignment due to image noise and artifacts. And, the computational time is relatively long.

Numerous methods have been proposed for image registration, e.g., non-rigid registration using FFD. In Rueckert's approach [110], global motion was modeled by an affine transformation, while local motion was described by an FFD based on multilevel B-splines. Registration was achieved by optimizing a cost function measuring the similarity between two images as well as the smoothness of the deformation needed to align the images. Chandrashekara et al. $[111,112]$ made use of the nonrigid registration algorithm first proposed in [110] and applied it to the analysis of myocardial deformations. The algorithm in [110] can only be applied to either SA or LA images, which means that through-plane motion could not be accounted for. In order to obtain complete 3D motion of the myocardium, the cost function was 
modified to be the sum of the normalized mutual information between the registered SA and LA images. The main advantage of this method is that tag localization and deformation field reconstruction were performed simultaneously. Another advantage is that no assumptions about the nature of the tag pattern are made so that the proposed method could potentially be applicable to untagged cine MR images or ultrasound images. However, the fact that no tag position information is incorporated could be considered a limitation. Rougon et al. [113] extended the similarity measure based on NMI to generalized information measures in order to quantitatively assess ventricular wall function from tagged MRI. Different from Chandrashekara's method, Ali-Silvey class of generalized information measures was used, which is a superset of MI and NMI. For more information about medical image registration, refer to the survey paper $[109,114]$.

\section{E. Frequency-based Methods}

\section{Harmonic Phase Analysis (HARP)}

Harmonic Phase (HARP) is a phase-based technique for rapid analysis of tagged cardiac MR images $[8,115,116]$. Rather than working on tagged images directly, HARP uses phase information in the frequency domain. It is based on the fact that the Fourier transform of a SPAMM tag image is a collection of distinct spectral peaks, each of which contains motion information in a certain direction. After bandpass filter, one spectral peak can be extracted and then applied an inverse Fourier transform, yielding the harmonic image. A harmonic image is a complex image that has magnitude and phase images, which is given in Equation (48). This process is shown in Figure 26 for simulated data and Figure 27 for real dog data. The HARP

method uses $\Phi_{k}(y, t)$, the phase of the harmonic image, which is called harmonic 

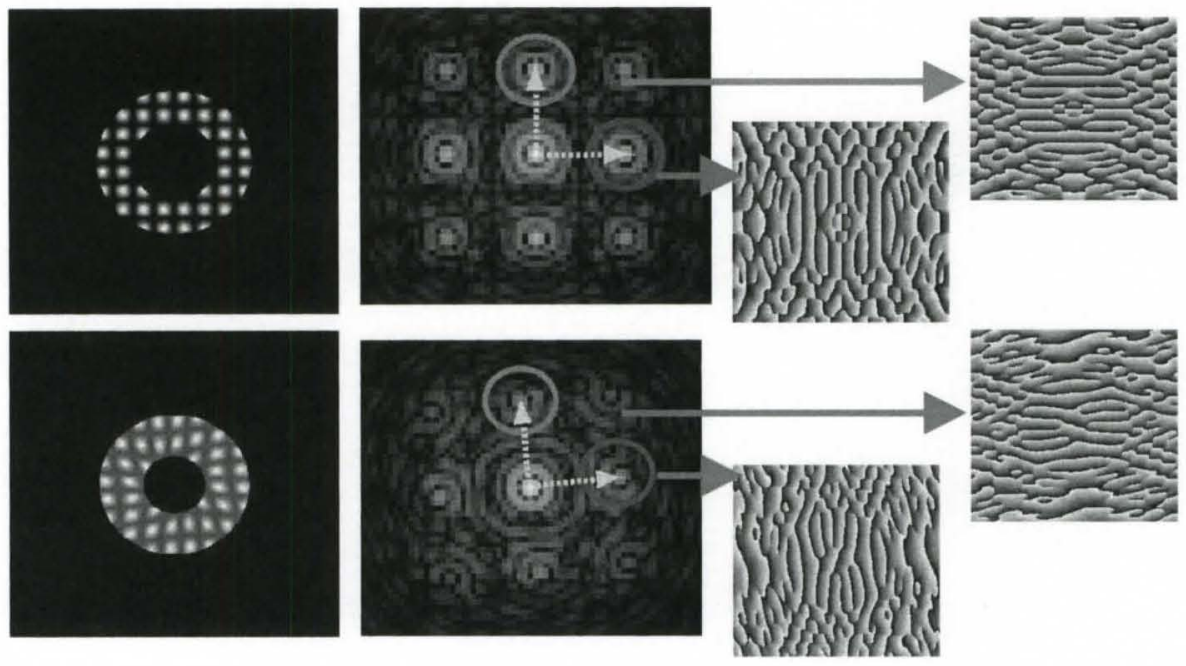

FIGURE 26 - Tagged images, their Fourier peaks, and their corresponding phase images

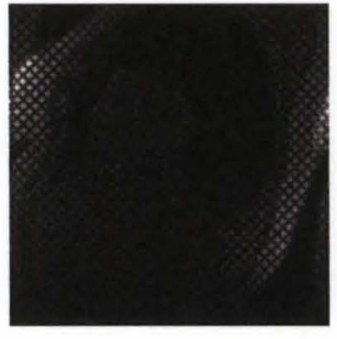

(a)

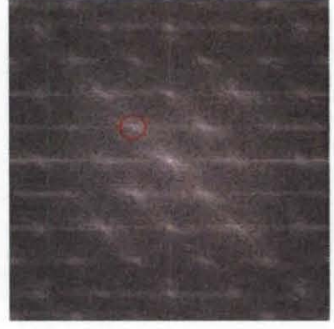

(b)

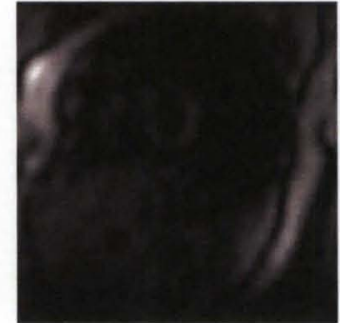

(c)

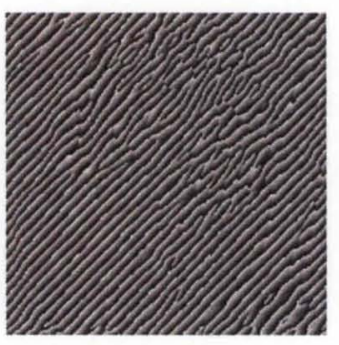

(d)

FIGURE 27-(a) An tagged MR image. (b) The magnitude of its Fourier transform. By extracting the spectral peak inside the circle in (b), a complex image is produced with a magnitude (c) and a phase (d).

phase image or simply HARP image. The harmonic phase image is linearly related to a directional component of the true motion.

$$
I_{k}(y, t)=D_{k}(y, t) e^{j \Phi_{k}(y, t)}
$$

An image taken immediately after the application of a 2-D SPAMM tag pattern can be represented by

$$
I(\mathbf{p})=I_{0}(\mathbf{p}) f\left(\mathbf{p} ; \mathbf{g}_{1}\right) f\left(\mathbf{p} ; \mathbf{g}_{2}\right)
$$

where $I_{0}$ is the image that would have been produced without tagging and $f$ is the 
tag pattern function:

$$
f(\mathbf{p} ; \mathbf{g})=\sum_{n=0}^{N-1} d_{n} \cos \left(n \mathbf{g}^{T} \mathbf{p}\right)
$$

where $d_{n}$ are coefficients determined by the sequence of tip angles and the gradient direction $\mathbf{g}$ is given by $\mathbf{g}=\gamma \int_{0}^{T} \mathbf{G}(t) d t$, where $\gamma$ is the gyromagnetic ratio. From Equation (49), it can be seen that it is an amplitude modulation of the underlying signal intensity by a pattern of cosines. Rewriting Equation (49) as

$$
I(\mathbf{p})=\sum_{k=1}^{K} I_{0}(\mathbf{p}) c_{k} e^{j \mathbf{w}_{k}^{T} \mathbf{p}}
$$

where $K=2 N-1$ for 1-D SPAMM and $K=(2 N-1)^{2}$ for 2-D SPAMM.

The image taken at time $t$ as a function of the spatial position can be represented as

$$
I(\mathbf{y}, t)=\sum_{k=1}^{K} I_{0}(\mathbf{p}(\mathbf{x}(\mathbf{y})), t) c_{k}(t) e^{j \mathbf{w}_{k}^{T} \mathbf{p}(\mathbf{x}(\mathbf{y}), t)}
$$

where $\mathbf{p}(\mathbf{x}, t)$ is the reference map at time $t$ and $\mathbf{x}, \mathbf{y}$ are the spatial position of a material point $\mathbf{p}$ and the image coordinates, respectively. $\mathbf{y}$ is related to $\mathbf{x}$ by

$$
\mathbf{x}(\mathbf{y})=y_{1} \mathbf{h}_{1}+y_{2} \mathbf{h}_{2}+\mathbf{x}_{0}
$$

where $\mathbf{h}_{1}$ and $\mathbf{h}_{2}$ are unit vectors describing the image orientation and $\mathbf{x}_{0}$ is the image origin. In Equation (52), a SPAMM-tagged image taken at time $t$ is the sum of $K$ complex images, each corresponding to a distinct spectral peak identified at the location $\mathbf{w}_{k}$.

Motion tracking using HARP depends on the fact that the phase of a point in the tissue is constant throughout a motion or deformation. The tracking algorithm searches for the point in a second image that has the same two phase values as the point of interest in the first image. This process is applied to the entire image sequence, yielding the motion trajectory for one selected point. Mathematically, for a given material point at $(x, y)$ at image slice $s$ at time $t$, if $\left(x^{\prime}, y^{\prime}\right)$ is the position at 
image slice $s$ at $t+1$, the phase time-invariance condition is expressed as follows. The principle of HARP tracking is that the harmonic phase of a point remains constant as it moves during the cardiac cycle. For a given material point $(x, y)$ at image slice $s$ at time $t$, which takes on position $\left(x^{\prime}, y^{\prime}\right)$ in the same slice at time $t+1$, the phase time-invariance condition is expressed as follows:

$$
\left\{\begin{array}{l}
P^{h}\left(x^{\prime}, y^{\prime}, s, t+1\right)=P^{h}(x, y, s, t), \\
P^{v}\left(x^{\prime}, y^{\prime}, s, t+1\right)=P^{v}(x, y, s, t)
\end{array}\right.
$$

where $x, y, s$, and $t$ stand for a point location in image plane, the short-axis image slice location, and the time frame, respectively.

According to this condition, 2-D displacement $\left(u_{s}^{t+1}, v_{s}^{t+1}\right)$ from time frame $t$ to $t+1$ at position $\left(x^{t}, y^{t}\right)$ in a short- or long-axis image slice location can be obtained by forcing matching points to satisfy Equation (54). Equation (54) is multidimensional and nonlinear and may be solved iteratively using the Newton-Raphson technique [8].

$$
y^{n+1}=y^{n}-\left[\nabla \phi\left(y^{n}, t_{m+1}\right)\right]^{-1}\left[\phi\left(y^{n}, t_{m+1}\right)-\phi\left(y_{m}, t_{m}\right)\right]
$$

where $\nabla$ is the gradient with respect to $y$ and $\phi\left(y_{m}, t_{m}\right)$ is the wrapped phase at time $t_{m}$.

By manipulating harmonic peaks, more desirable tag patterns from conventional SPAMM, CSPAMM, and fast-HARP tagged images can be synthesized [117]. In order to correct errors in harmonic phase images, Ryf et al. [118] employed both negative and positive peaks to produce more accurate harmonic phase images. The main advantage of HARP is that it is fast and automatic with no need of extracting tag lines as a preprocess step. One problem with HARP is that it can fail in the presence of a large amount of motion. To address this problem, Liu et al. proposed a seed-region growing based algorithm [119] and a shortest path method [120] for HARP refinement.

Although HARP is fast in postprocessing tagged MR images, the time needed to acquire a cine sequence of tagged images is on the order of 8 to 20 heartbeats 
during a breath-hold. Based on the fact that only two spectral peaks are needed to compute 2D deformation, Sampath et al. $[121,122]$ presented a pulse sequence to only acquire a small region in k-space around the selected spectral peaks after tagging. This approach reduces the acquisition time and provides the possibility for quantifying regional function of the heart in real time. Using this fast-HARP pulse sequence, real time myocardial strains can be computed in 2D [123]. By using chirp inverse Fourier transform, myocardial ROI was cropped from the raw k-space data so that image reconstruction and cardiac strain map computation could be accomplished in $11 \mathrm{~ms}$. This method can produce the same results as original HARP with a 4 times speedup.

One limitation of HARP and fast-HARP is that they can only estimate the in-plane motion, typically using 2D SA tagged MR images. Several solutions for including the out-plane component of motion have been proposed. Pan et al. [124] extended the traditional HARP method for 3D cardiac motion tracking. A stack of SA images with tagging grid as well as LA images with one horizontal set of tags were used. Similar to 2D HARP, phase invariant condition was used to track the motion. A material mesh model was used for interpolation from sparse phase information. In this study, only a single layer mesh inside the myocardium was created and tracked according to $3 \mathrm{D}$ phase invariant condition. Using a true $3 \mathrm{D}$ tagging technique followed by a conventional fast 3D gradient echo imaging sequence, Ryf et al. [125] extended HARP from $2 \mathrm{D}$ to $3 \mathrm{D}$. The phase invariant property in three directions was utilized after extracting spectral peaks in 3D Fourier space. True 3D motion can be captured, but the scan time was quite long and demanded a sophisticated breath-holding technique. Rutz et al. proposed a fast method for acquiring 3D CSPAMM data using localized tagging preparation and a hybrid multishot, segmented echo planar imaging sequence [11]. Abd-Elmoniem et al. [126] developed a new method, zHARP pulse sequence, which can track both in-plane and through-plane motion from a single image 
plane. It is similar to slice-following CSPAMM [127] and combines tagged MRI with through-plane displacement phase encoding without affecting the image acquisition time. A z-encoding gradient is added to the refocusing lobe of the slice-selection gradient and produces a phase term depending on the displacement in through-plane direction in the final data. From two or more images in one orientation, 3D strain can be calculated [128]. zHARP can compute 3D strain tensor over the whole LV with no spatial interpolation. Another method to obtain through-plane strain as well as in-plane strain is to combine fast-HARP with SENC [70,129]. Using the HARPSENC pulse sequence, a single slice of SA image can be acquired in six heartbeats and multiple slices can be acquired in a sequential fashion.

\section{Local Sine Wave Modeling}

SinMod is also a frequency-based method to analyze the heart displacement and deformation from tagged MRI sequences using phase information [9]. The main difference between SinMod and HARP is that SinMod detects both local spatial phase shift and local spatial frequency from band-pass filtered images, while HARP uses the phase invariant condition and tracks local spatial phase. The speed of SinMod method is as fast as HARP but SinMod method has advantages in accuracy, noise reduction, and lack of artifacts [9]. In SinMod, the intensity distribution around each pixel $(p, q)$ is modeled as a cosine wave front.

$$
\begin{aligned}
& I_{1}(p, q)=A_{1} \cos \left(\omega_{p}\left(p+\frac{u}{2}\right)+\varphi\right)+n_{1}(p, q) \\
& I_{2}(p, q)=A_{2} \cos \left(\omega_{p}\left(p-\frac{u}{2}\right)+\varphi\right)+n_{2}(p, q)
\end{aligned}
$$

where $\omega_{p}$ and $\varphi$ are the spatial frequency and phase of the wave, respectively. $A_{1}$ and $A_{2}$ are wave magnitudes for the first image $I_{1}$ and the second image $I_{2}$, while $n_{1}$ and $n_{2}$ are additive noise. $u$ is the displacement between these two images at position $(p, q)$ along the $p$ direction. 
The flow chart of SinMod algorithm is shown in Figure 28. The principle behind SinMod tracking is that both phase and frequency for each pixel are determined directly from the frequency analysis and the displacement is calculated from the quotient of phase difference and local frequency. After obtaining the Fourier Transform of the input images $I_{1}(p, q)$ and $I_{2}(p, q)$ (temporal frames at time $t$ and time $t+1$ ), the same band-pass filter is applied to both (similar to HARP) to isolate corresponding spectral peaks and produce a pair of complex images in the Fourier domain (since the Fourier Transform will be complex to begin with). The two complex images in Fourier domain following band-pass filtering are noted as $I_{b f 1}\left(\omega_{p}, \omega_{q}\right)$ and $I_{b f 2}\left(\omega_{p}, \omega_{q}\right)$. Applying a low frequency band-pass filter and a high frequency one to both $I_{b f 1}$ and $I_{b f 2}$ followed by an inverse Fourier transform leads to four complex images $I_{b f L f 1}(p, q)$, $I_{b f H f 1}(p, q), I_{b f L f 2}(p, q)$, and $I_{b f H f 2}(p, q)$. The reasoning behind application of a LPF and a HPF to $I_{b f 1}$ and $I_{b f 2}$ is to determine the local spatial frequency by power spectra. Then the displacement is the local quotient of phase difference and local frequency at that position. The power spectra and cross power spectrum are given by:

$$
\begin{aligned}
& P_{L f}(p, q)=\left|I_{b f L f 1}\right|^{2}+\left|I_{b f L f 2}\right|^{2} \\
& P_{H f}(p, q)=\left|I_{b f H f 1}\right|^{2}+\left|I_{b f H f 2}\right|^{2} \\
& P_{c c}(p, q)=I_{b f L f 1} \bar{I}_{b f L f 2}+I_{b f H f 1} \bar{I}_{b f H f 2}
\end{aligned}
$$

where $\bar{I}$ is the complex conjugation of $I$.

The local frequency $\omega_{p}$ and local displacement $u$ can then be estimated from:

$$
\omega_{p}(p, q)=\omega_{c} \sqrt{\frac{P_{H f}}{P_{L f}}} \quad u(p, q)=\frac{\arg \left(P_{c c}\right)}{\omega_{p}}
$$

where $\omega_{c}$ is the band-pass center-frequency. 


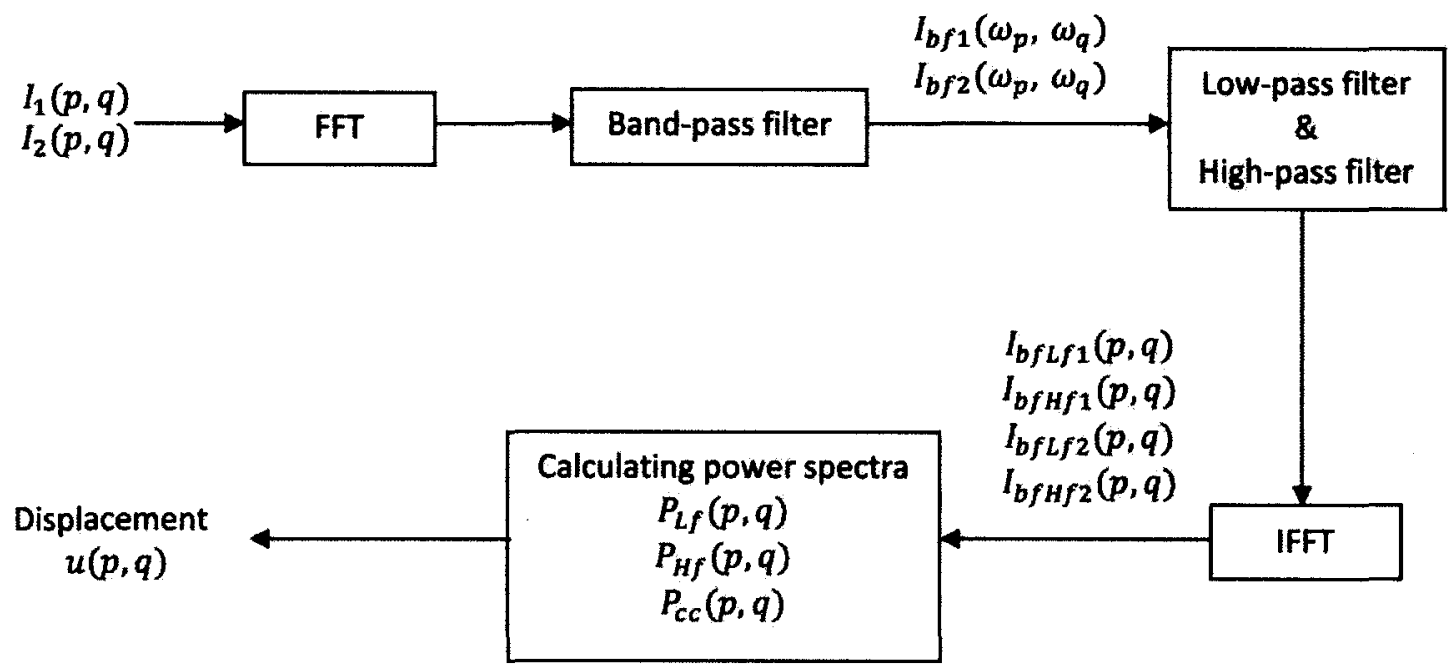

FIGURE 28-Flow chart for the SinMod method

3. Gabor Filter Banks

Gabor filter is a band-pass filter, with the form of a Gaussian multiplied by a complex sinusoid in the spatial domain, or equivalently, a shifted Gaussian in the spatial frequency domain. By choosing appropriate parameters, the magnitude response of the Gabor filter can be used to remove tags in the myocardium [130], and the phase response can be used to track tags. Once again, utilizing phase-based optical flow, the phase response of Gabor filter bank for a material point is assumed to be constant during the deformation [131].

A Gabor filter bank is a set of $2 \mathrm{D}$ Gabor filters with tunable parameters that represent the variable spacing and orientation of tag lines. By finding optimal parameters that maximize the Gabor filter response, Montillo et al. [132] extracted deformation information in a 2D simulated model. Qian et al. [133] extended the 2D Gabor filter bank method to 3D in order to extract and track deformed tag surfaces. Chen et al. [131] used the phase information from Gabor filters to track tag lines. To increase the accuracy of tag tracking, a combination of the response of Gabor filters, 
gradient information of original images, an intensity probabilistic model, and a spatio-

temporal smoothness constraint was used in the deformable model. Chen et al. [134] proposed a three-step process for 3D cardiac motion tracking: extract tag intersections based on local phase analysis using Gabor filter bank, robust point matching (RPM) method to track the intersections movement, and meshless deformable modeling to generate a dense displacement field.

Instead of calculating the strain values in terms of gradient of the displacement by tracking the tag pattern, Qian et al. [135] developed a non-tracking-based strain estimation method for tagged MRI. It is based on the extraction of tag's deformation gradient using Gabor filter, instead of tracking displacement prior to strain calculation. Subsequently, 2D strains can be obtained by using the strain formula in terms of the deformation gradient tensor.

\section{F. Strain Pattern Analysis}

An important application of MRI tagging is in providing a better definition of the normal patterns of motion. Similarly, the effects of heart diseases such as hypertrophy, infarction, failure, and dyssynchrony can be well-characterized and quantified with tagging.

Regional myocardial strains have direct or indirect relationship with cardiac diseases. Most of the motion tracking methods aim to extract strains in the heart, for the reason that strain encapsulates the basic mechanical function of the myocardium and has clinical potential. There are different types of strains. Normal strain is defined as the ratio of the length of the deformed line element to its original length. Due to the geometry of the ventricle, normal strains are usually calculated in radial, circumferential, and longitudinal directions. Shear strain is defined as the angular change between any two originally mutually orthogonal line elements that occurs as a result of a continuous deformation. These can be defined in terms of the off-diagonal 
terms of the Lagrangian strain tensor [13].

Strain analysis can also be done based on extracted model parameter functions [84] where quantitative differences between normal and abnormal hearts with hypertrophic cardiomyopathy (htcm) were extracted. Park et al. [84] showed that the contraction and the twisting deformation are more significant on the endocardial wall compared to the epicardial wall. Due to the complexity of RV geometry, Haber et al. [88] utilized coordinate-system-independent principal strains and directions to describe regional deformations, which were derived by calculating the eigenvalues and eigenvectors of the Lagrangian strain tensor. The strains in normal hearts and the hearts with right ventricular hypertrophy (RVH) diseases were compared. Maximum and minimum principal strains were used to quantify normal sheep left ventricular functions [105].

Normal strains have been widely used, in radial, circumferential, and longitudinal directions, though the circumferential strain values have been reported most $[8,85,97,122,124,134]$. In [78], circumferential strains were computed, not for every material points, but based on the percent change in the distance between every set of two adjacent markers with respect to the reference time frame. In [70], radial, circumferential, and longitudinal strains (in-plane and out-of-plane strains) were obtained simultaneously within a mid-ventricular SA slice. In [99], circumferential, radial, and longitudinal strains for a normal volunteer and a patient with an antero-septal myocardial infarction were computed. In [9], circumferential strains of the LV in five slices from apex to base and the mid-wall circumferential strain for a patient with left bundle branch block (LBBB) and infarction in the lateral LV-free wall were investigated.

Moore et al. $[136,137]$ reported 3D displacement and strain evolution of the normal LV in humans determined from tagged MRI. Such data can serve as a database of strain in normal hearts. Clarysse et al. [138] proposed a framework for analysis 
of spatiotemporal deformation parameters of the myocardium throughout the systolic phase from tagged MR data. After strain calculation, a functional data analysis method was applied to healthy subjects, which provided a normal contraction reference model. Pathological cases were then compared with the reference model. Statistical analysis of regional wall motion performance parameters in normals relative to disease processes was done in [3]. After the deformation was reconstructed based on FEM models fitted to tagged MR images [102], the deformation of LV can be decomposed into separate deformation modes such as longitudinal shortening, wall thickening, and twisting [139]. By doing this, healthy and diseased cardiac deformation patterns can be distinguished.

Cupps et al. [140] estimated LV wall stress distribution at rest in patients with chronic aortic insufficiency and normal ejection fraction within an FEM formulation. Differences in regional LV systolic stress in normal subjects and patients with aortic insufficiency were given. Moustakidis et al. [141] estimated 3D strain distribution at rest and with inotropic stimulation in patients with ischemic cardiomyopathy using tagged MR imaging and FE analysis. Similar studies were performed in a group of normal volunteers for comparison. Further study showed that circumferential strains at rest and with low-dose dobutamine could discriminate viable and nonviable myocardium in patients with ischemic cardiomyopathy [142]. Maniar et al. [143] compared circumferential strain in patients before and after 3 months after coronary artery bypass grafting using tagged MRI. Strain analysis from dobutaminestressed tagged MRI was shown to be feasible to quantitatively predict myocardial recoverability after coronary artery bypass grafting [144]. Chen et al. [134] assessed the differences in myocardial strain distributions between hearts with left ventricular hypertrophy and normal hearts. They calculated statistics of 17 different variables related to the circumferential strain distribution in the LVH patients and the normal control. 
In this chapter, different image analysis methods to evaluate cardiac function from tagged MRI were reviewed. In summary, there are two different categories of cardiac motion analysis methods using tagged MRI. The first category is feature-based motion tracking methods, i.e., those that measure the deformations by tracking the movement of tagging features. The most commonly used features are sparse tag lines, geometrically salient land markers, tag line intersections, as well as epicardial and endocardial contours. A number of papers have been published on how to detect tag lines and contours $[130,145,146]$. For tracking, two sets of techniques have emerged. One set of techniques is to track the features without constructing a dense motion field. The other set constructs a dense motion field from the sparse motion field obtained by tracking the tag features. The accuracy of feature-based image analysis methods using tagged MRI depends highly on the quality of the image and the spacing of tag lines. Tagged MR images with higher spacial resolution will provide more information and constraints on the models. Any advances and improvements in MR imaging, tagging techniques, tag detecting methods, and modeling methods will have impact on another. The second category aims to obtain the deformation field directly from tagged MR images, without extracting tag features. Three different types of methods fall in this category: optical flow methods, registration-based methods [111113], and frequency-based methods, for example, HARP $[8,115]$ and SinMod [9]. 


\section{CHAPTER V \\ 2D TAGGED MRI ANALYSIS WITH MULTILEVEL B-SPLINES}

A number of approaches have been proposed in recent years for analysis of cardiac motion from tagged MRI (See Chapter IV). Among various approaches,

B-splines have been used extensively in temporal registration and reconstruction of myocardial deformations due to their ability to conform to local deformations while enforcing continuity. By considering the real tag intersections (in the spatial domain) and virtual tag intersections (from the frequency domain) as scattered data, multilevel B-splines (MBS) can result in accurate and fast approximations without the need to specify the control point locations explicitly. The contents of this chapter are based on two papers that have been published in the SPIE Medical Imaging conference $[6,7]$.

In this chapter, a method that combines the advantages of continuity and smoothness of MBS has been developed. It makes use of phase information derived from frequency-based analysis of tagged MR images. Compared to normal B-spline method, MBS [147] have the following advantages: 1) They can simultaneously achieve a smooth shape while accurately approximating the given data set; 2 ) Computationally, they are very fast; and 3) They can produce more accurate deformation results. Since the tag intersections (intersections between two tag lines) can be extracted accurately [130] and are more or less distributed evenly over the domain, MBS are effective for cardiac motion tracking. By producing virtual tags from HARP or SinMod and calculating the resulting intersections, more data points may be obtained. Virtual tag lines at the reference frame are the isoparametric curves of an undeformed 2D B-spline model. For subsequent frames, the intersections of virtual tag lines over the domain are updated by phase-based displacement. The basic idea of 


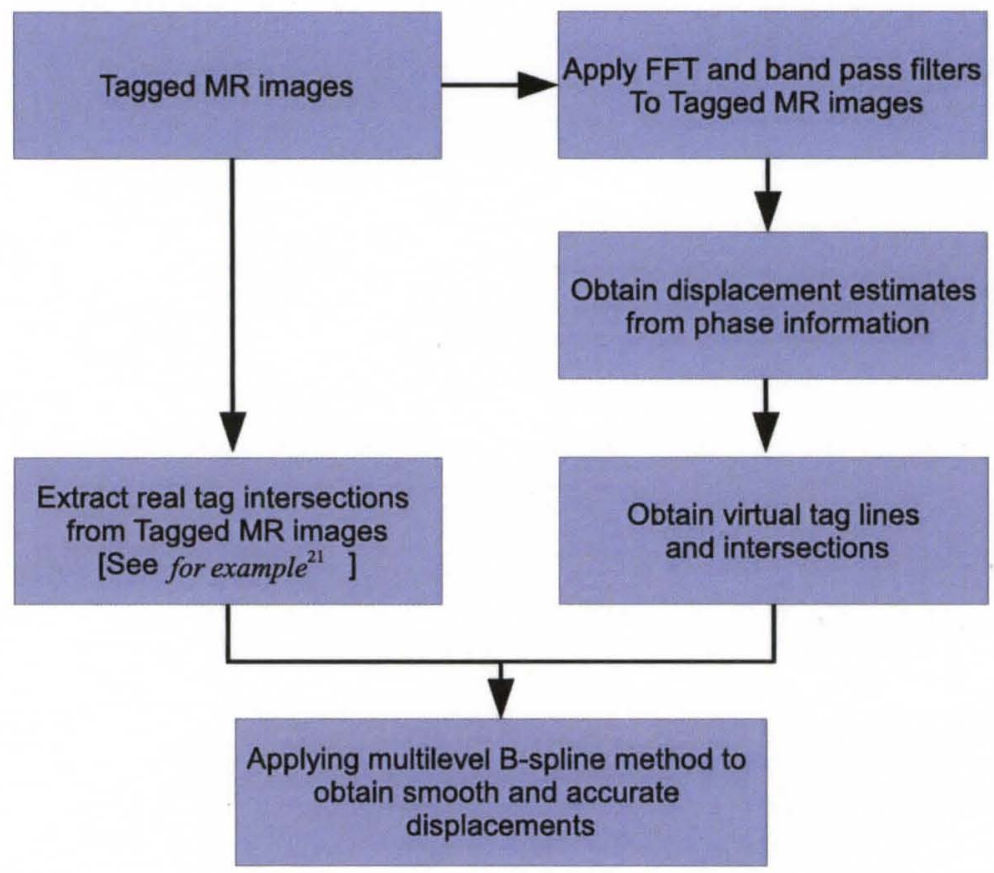

FIGURE 29-Overview of the proposed multilevel B-splines method

the paper is to use both real tag line intersections as well as virtual ones from phasebased tracking to produce dense myocardial displacements. The proposed method takes advantage of the multilevel B-spline scattered data fitting method. It is fast, precise, and more accurate than HARP tracking. Figure 29 displays the overview of the proposed method.

\section{A. Multilevel B-Splines With Phase Information}

MBS [147] can be used to generate a coarse-to-fine sequence of tensor product B-splines whose sum approaches the final approximation of a given scattered data. The method works locally because on the finer tensor product grids, each B-spline coefficient is computed from neighboring points alone. Consequently, the method is extremely fast compared to other methods. The deformed position, $q$ of any arbitrary 
point with local coordinates $(u, v)$, is given by

$$
q(u, v)=\sum_{m=0}^{3} \sum_{n=0}^{3} p_{i+m, j+n} N_{m, 3}(u) N_{n, 3}(v)
$$

where $p_{i, j}$ is the $i^{\text {th }}, j^{\text {th }}$ control point in the $u, v$ direction, respectively, and $N_{m, 3}, N_{n, 3}$ are the uniform cubic B-spline basis functions defined by

$$
\begin{aligned}
& N_{0,3}(x)=(1-x)^{3} / 6, \\
& N_{1,3}(x)=\left(3 x^{3}-6 x^{2}+4\right) / 6, \\
& N_{2,3}(x)=\left(-3 x^{3}+3 x^{2}+3 x+1\right) / 6, \\
& N_{3,3}(x)=x^{3} / 6
\end{aligned}
$$

where $0 \leq x<1$.

The algorithm utilizes a hierarchy of control lattices to generate a sequence of functions $f_{k}$ and the desired approximation function is achieved by summing all the $f_{k}^{\prime} \mathrm{s}$ (Figure 30). Therefore, following an initial fit with the $\phi_{i}$ control lattice, the $\phi_{i+1}$ control lattice is used to find an approximation to the remaining error of fit. The multilevel B-spline method takes a set of scattered data as input and produces tensor product B-spline surfaces as output. The final surface $f$ is composed of a sequence of surfaces at different scales,

$$
f=f_{0}+f_{1}+\cdots+f_{n}
$$

The proposed method takes advantage of both phase-based motion tracking method and multilevel B-spline approximation, as illustrated in Figure 29. First, Fourier transform is apply to the tagged MR images and obtain displacement fields from phase information. Then, tag lines in the image frames are detected and located under a MAP Framework using Markov Random Fields [130]. After extracting the tag lines, the intersections of short-axis tag lines and the intersections between tag lines and epicardial and endocardial contours are determined. The real tag intersection correspondences are obtained automatically when the tag lines in the region 


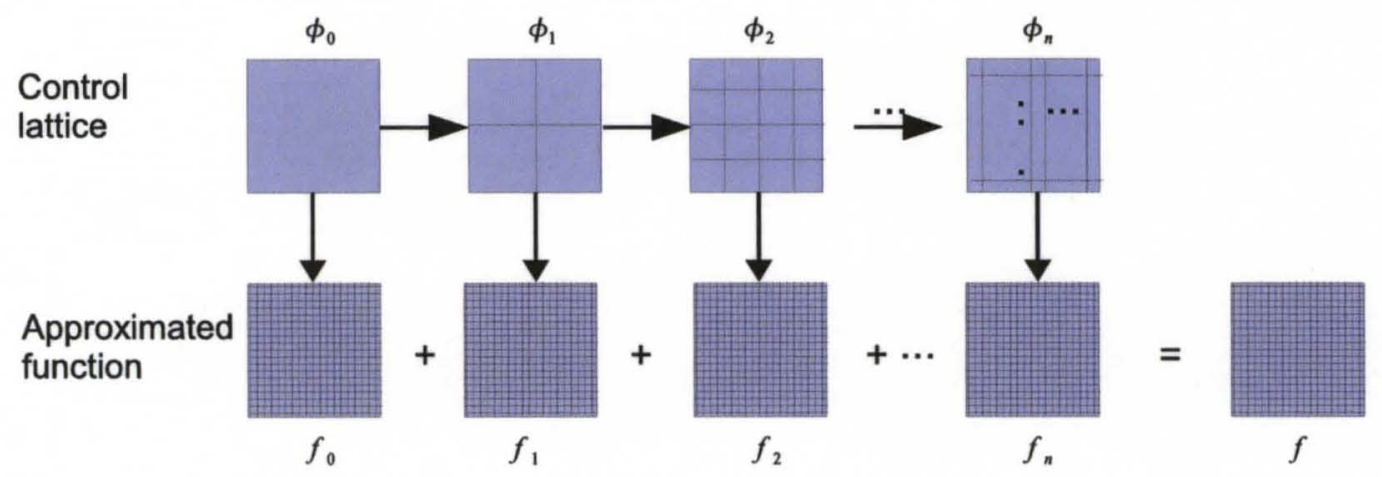

FIGURE 30 -Multilevel B-spline Hierarchy

of interest are represented by a B-spline grid. Due to the sparsity of real tag intersections from tagged MRI data, dense virtual tag lines intersections obtained from phase-based displacement estimation are additionally utilized to reconstruct deformation fields. Virtual tag line intersections are generated in the following way: after placing virtual tag line intersections in the reference frame (end-diastole), all the corresponding locations of these intersections in subsequent time frames can be obtained through phase-based motion tracking, as illustrated in Figure 31. After one-to-one correspondences for both real and virtual tag line intersections are established in different time frames, for example, pairs of points $(P i, Q i)$, this breaks down into two scattered data interpolation problems: one for component pairs $\left(P i, Q i_{x}\right)$ and one for pairs $\left(P i, Q i_{y}\right)$. Pi, Qi are corresponding landmark points in two frames. The transformation represented by B-spline control points is obtained by minimizing the residual errors between $x$ coordinates and separately the $y$ coordinates of corresponding points in two subsequent frames. The resulting transformation brings real tag line intersections as well as virtual ones into alignment. Finally, a B-spline fitting model is established, which produces the optimal locations for B-spline control points from which a dense displacement vector field is calculated. 

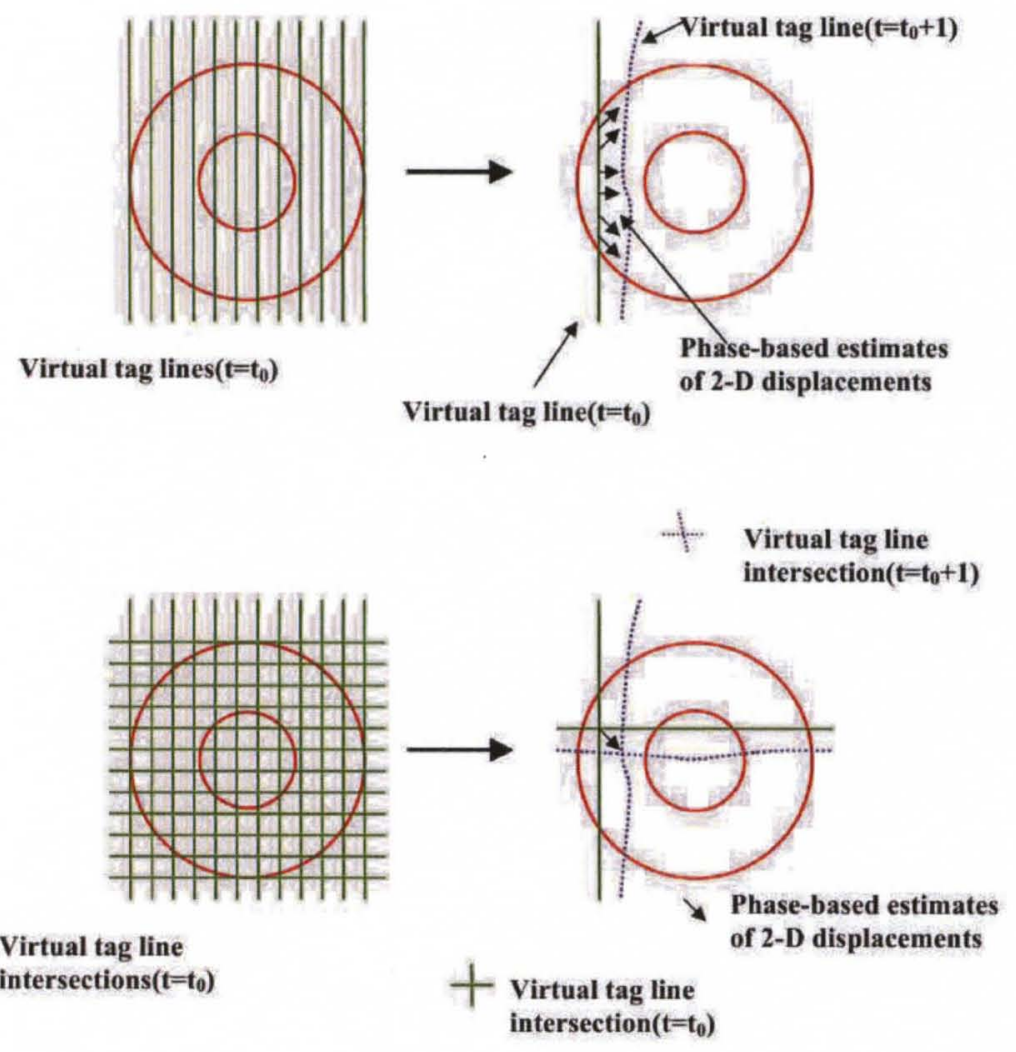

FIGURE 31 - Creating virtual tag lines and tag intersections. Column 1: virtual tag lines placed in the reference frame (solid green lines). Column 2: the motion field that warps the virtual tag lines (dashed blue lines). 
Starting from a $4 \times 4$ control grid, which is a single B-spline span, the positions of control points are updated iteratively by minimization of the local residual error at tag intersections until the tag line intersections are aligned. To optimize the location of B-spline control points, the least-square solution is computed in each iteration such that the sum of squared distances between tag intersections in frame 1 and its corresponding deformed locations in frame 2 is minimized. With multilevel deformation, the number of deformation parameters are increased gradually and the matching moves from coarse to fine levels (See Figure 30).

\section{B. Results}

In order to validate the accuracy of the proposed method, simulated data were generated from the 13-parameter kinematic model of Arts et al. [148]. The importance of the simulator is that it is able to generate both simulated tagged cardiac MR images reflecting the motion of the heart model as well as the ground-truth motion field of myocardial deformations. This allows to compare the motion field predicted from the proposed approach using the simulated tagged MR images with the true motion field. In the experiments, the parameters $k_{1}, k_{2}, k_{3}$ were varied in order to generate different levels of radially dependent compression, left ventricular torsion, and long axis ellipticalization. The parameter values used in this paper are the same as those in Tustison's thesis [17] and are also shown in Table 1. The simulated data consisted of eleven frames of eight short-axis images. In this chapter, we considered the images in the systolic phase, the first five frames at three slice locations. Figure 32 demonstrates sample short-axis images of the simulated data.

To assess tracking performance of different methods, the distance between the

ground truth tag grid $\hat{G} 1$ and deformed tag grid $G 2$ was measured when $\hat{G} 1$ is warped 


\section{TABLE 1}

The values of the thirteen $k$ parameters of the cardiac simulator for the simulated data.

\begin{tabular}{|c|c|c|c|c|c|c|}
\hline Frame & 1 & 2 & 3 & 4 & 5 & 6 \\
\hline$k_{1}$ & 0.0 & -0.1224 & -0.1987 & -0.2386 & -0.2500 & -0.2391 \\
\hline$k_{2}$ & 0.0 & 0.1468 & 0.2384 & 0.2864 & 0.3000 & 0.2870 \\
\hline$k_{3}$ & 0.0 & -0.0245 & -0.0398 & -0.0478 & -0.0500 & -0.0479 \\
\hline$k_{4}-k_{13}$ & 0.0 & 0.0 & 0.0 & 0.0 & 0.0 & 0.0 \\
\hline \hline Frame & 7 & 8 & 9 & 10 & 11 & \\
\hline$k_{1}$ & -0.2112 & -0.1703 & -0.1198 & -0.0624 & 0.0 & \\
\hline$k_{2}$ & 0.2535 & 0.2044 & 0.1438 & 0.0748 & 0.0 & \\
\hline$k_{3}$ & -0.0423 & -0.0341 & -0.0240 & -0.0125 & 0.0 & \\
\hline$k_{4}-k_{13}$ & 0.0 & 0.0 & 0.0 & 0.0 & 0.0 & \\
\hline
\end{tabular}

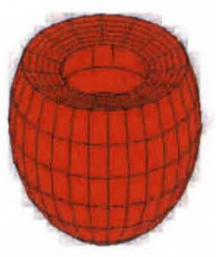

(a)

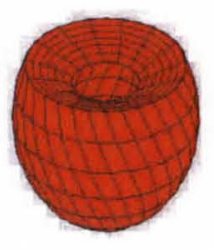

(b)

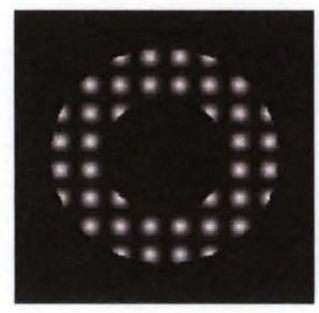

(c)

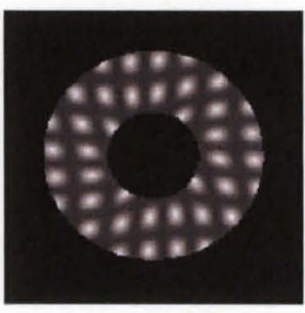

(d)

FIGURE 32 - A realistic simulation. A 3-D solid model of the heart from Arts' Model at (a) end-diastole and (b) end-systole. Short-axis simulated images at (c) enddiastole and (d) end-systole. 
by the motion field produced by that method. The distance metric used was [91]:

$$
d(\hat{G} 1, G 2)=\frac{1}{N} \sum_{k=1}^{N} \frac{1}{2}\left\{\max \min D\left(\hat{G} 1_{k}, G 2_{k}\right)+\max \min D\left(G 2_{k}, \hat{G} 1_{k}\right)\right\}
$$

where $\hat{G} 1_{k}, G 2_{k}$ are the $k^{\text {th }}$ tag curves (horizontal or vertical), and $D$ is the Euclidean distance metric. maxmin $D\left(\hat{G} 1_{k}, G 2_{k}\right)$ is a scalar measure of the distance from curve $\hat{G} 1_{k}$ to curve $G 2_{k}$, the maximum value among the Euclidean distances of points on curve $\hat{G} 1_{k}$ to curve $G 2_{k}$. Note that the distance from curve $\hat{G} 1_{k}$ to curve $G 2_{k}$ is not necessarily the same as the distance from curve $G 2_{k}$ to curve $\hat{G} 1_{k}$. Table 2 compares the three approaches using the metric of equation (61) for simulated data and Table 3 compares the same three methods on systolic mid-ventricular tagged MRI data from a normal canine (See Figure 33).

The results for simulated data are shown in Table 2. Note that for simulated data, all the distances are between tag grid deformed by the ground truth motion field and motion field from the method being evaluated. As seen, the tag grid distances from the MBS method are smaller than those from HARP and SinMod, showing the accuracy and effectiveness of MBS.

The relative root mean squared (RRMS), root mean squared (RMS) error, average length error, and average angle error were employed to measure the accuracy of computed motion fields:

$$
\begin{aligned}
& \epsilon_{r r m s}=\frac{\sqrt{\frac{1}{N} \sum_{\Omega}\left|V_{g}-V_{c}\right|^{2}}}{\frac{1}{N} \sum_{\Omega}\left|V_{g}\right|} \times 100 \% \\
& \epsilon_{r m s}=\sqrt{\frac{1}{N} \sum_{\Omega}\left|V_{g}-V_{c}\right|^{2}}
\end{aligned}
$$

where $V_{g}$ and $V_{c}$ are the ground truth and computed displacements of the same material point, $\Omega$ is myocardial region, and $N$ is the number of vectors.

The average angle, length error and RRMS between the ground-truth motion field and motion fields from HARP, SinMod, and MBS methods for the simulated data 
TABLE 2

Comparison of HARP, SinMod, and MBS on simulated data using the metric in equation (61). Distances are in pixels.

\begin{tabular}{|c|c|c|c|}
\hline Frame Numbers & HARP & SinMod & MBS \\
\hline Frame 1-2 & 1.2579 & 1.3001 & 1.1226 \\
\hline Frame 1-3 & 1.6313 & 1.6895 & 1.2980 \\
\hline Frame 1-4 & 2.1999 & 2.0105 & 1.4808 \\
\hline Frame 1-5 & 2.1898 & 1.9250 & 1.5061 \\
\hline
\end{tabular}

are given in Table 4. The average angle error, length error and RRMS using multilevel B-spline method are smaller than those calculated from HARP and SinMod. Similar results are shown in Table 5 for circumferential strains.

Note that since ground truth motion field is not available for in vivo data, tag lines were extracted manually and were used to compare motion fields from HARP, SinMod, and MBS based on Equation (61). From the comparison between HARP and SinMod for both simulated data and in vivo canine data, it can be seen that their performances are quite similar. SinMod is slightly better than HARP in general [9], but not for all the cases. For the experiments in this paper, phase tracking from HARP is used to produce the virtual tag line intersections. In the studies, one virtual tag line was inserted between every two real tag lines within the myocardium. An extensive study will be undertaken in the near future to determine the optimal number of virtual tag intersections that should be inserted, perhaps leading to further improvement in the results.

The first two frames of the simulated data and the first two frames of the canine study slice 4 together with the corresponding motion fields are shown in Figure 33. In this chapter, a multilevel B-spline fitting method (MBS) which incorporates 
TABLE 3

Comparison of HARP, SinMod, and MBS on mid-ventricular systolic tagged data from a normal canine using the metric in Equation (61). Distances are in pixels.

\begin{tabular}{|c|c|c|c|c|}
\hline Slice Number & Frame Number & HARP & SinMode & MBS \\
\hline \multirow{3}{*}{ Slice 3 } & Frame 1-2 & 1.01 & 0.96 & 0.67 \\
\cline { 2 - 5 } & Frame 1-3 & 1.09 & 0.93 & 0.75 \\
\cline { 2 - 5 } & Frame 1-4 & 1.14 & 1.10 & 0.96 \\
\hline \multirow{4}{*}{ Slice 4 } & Frame 1-2 & 0.88 & 0.88 & 0.84 \\
\cline { 2 - 5 } & Frame 1-3 & 1.50 & 1.38 & 1.02 \\
\cline { 2 - 5 } & Frame 1-4 & 1.23 & 1.13 & 0.94 \\
\hline \multirow{3}{*}{ Slice 5 } & Frame 1-2 & 1.04 & 0.90 & 0.70 \\
\cline { 2 - 5 } & Frame 1-3 & 1.13 & 1.07 & 0.63 \\
\cline { 2 - 5 } & Frame 1-4 & 1.23 & 1.18 & 0.75 \\
\hline
\end{tabular}

TABLE 4

Average angle, length error and RRMS between ground truth motion field and motion field from HARP, SinMod, and MBS for simulated data.

\begin{tabular}{|r|c|c|c|c|c|c|c|c|c|}
\hline $\begin{array}{r}\text { Frame } \\
\text { Numbers }\end{array}$ & \multicolumn{3}{|c|}{ HARP } & \multicolumn{3}{c|}{ SinMod } & \multicolumn{3}{c|}{ MBS } \\
\hline & Angle & Length & RRMS & Angle & Length & RRMS & Angle & Length & RRMS \\
\hline Frame 1-2 & 0.2306 & 0.3893 & 14.15 & 0.1682 & 0.3753 & 15.40 & 0.1747 & 0.3537 & 14.31 \\
\hline Frame 1-3 & 0.1108 & 0.8492 & 15.90 & 0.1493 & 0.5958 & 15.91 & 0.1097 & 0.7080 & 14.31 \\
\hline Frame 1-4 & 0.1931 & 1.1660 & 18.40 & 0.1385 & 0.7706 & 15.35 & 0.1063 & 0.6596 & 13.36 \\
\hline Frame 1-5 & 0.1712 & 1.2629 & 18.57 & 0.1513 & 0.8834 & 15.43 & 0.1013 & 0.6321 & 10.38 \\
\hline
\end{tabular}




\section{TABLE 5}

RMS error between circumferential strains computed from the ground truth motion field and motion fields from HARP, SinMod, and MBS for simulated data.

\begin{tabular}{|c|c|c|c|}
\hline Frame Numbers & HARP & SinMod & MBS \\
\hline Frame 1-2 & 0.0370 & 0.0448 & 0.0446 \\
\hline Frame 1-3 & 0.0675 & 0.0643 & 0.0476 \\
\hline Frame 1-4 & 0.0517 & 0.0638 & 0.0480 \\
\hline Frame 1-5 & 0.0579 & 0.0672 & 0.0428 \\
\hline
\end{tabular}

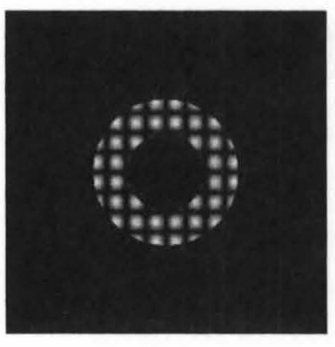

(a)

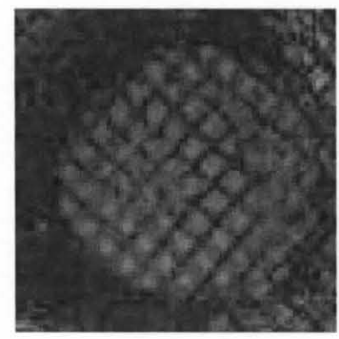

(d)

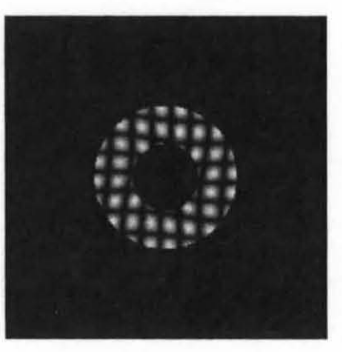

(b)

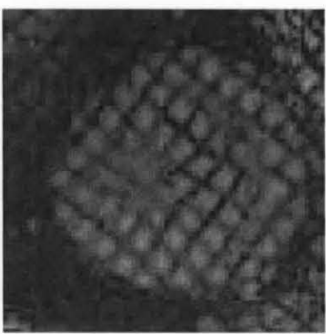

(e)

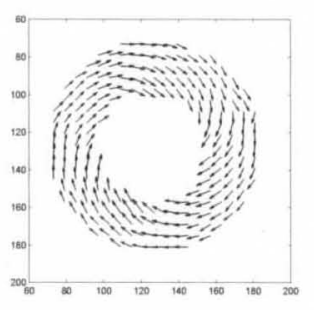

(c)

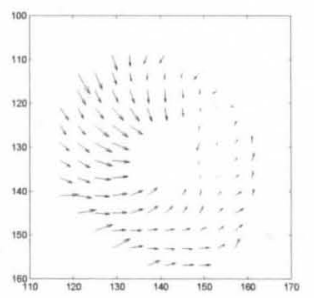

(f)

FIGURE 33-Tagged MR images and corresponding motion field between first two frames for both simulated and canine data. Canine data is from slice 4. (a)reference simulator image, (b) deformed simulator image, (c) motion field between (a) and (b), (d)reference canine image, (e)deformed canine image, (f) motion field between (d) and (e). 
phase displacement information for calculation of motion and strain from tagged MRI has been proposed. MBS uses both real and virtual tag intersections to derive motion fields and has several advantages: First, B-spline inherent characteristics guarantee the smoothness of the recovered motion fields. Second, it results in fast, accurate fitting. Third, incorporation of phase information helps overcome the sparsity of real tag intersections. The proposed method has been validated on simulated and in vivo tagged canine MRI data. Experimental results show better performance compared to HARP and SinMod techniques. 


\section{CHAPTER VI \\ ORTHOGONAL CSPAMM (OCSPAMM) MR TAGGING FOR IMAGING VENTRICULAR WALL MOTION}

Magnetic resonance imaging (MRI) is a highly advanced and sophisticated imaging modality for cardiac motion assessment and quantitative analysis. The

SPAMM myocardial tagging technique is widely available commercially $[27,28]$ and is the most commonly used technique for producing sinusoidal tag patterns. SPAMM suffers from tag fading in the later phases of the cardiac cycle due to $T_{1}$ relaxation. Complementary SPAMM (CSPAMM) was introduced by Fischer et al. in order to mitigate the tag fading problem [44]. Although highly effective, one disadvantage of CSPAMM as originally proposed is that it doubles the image acquisition time, since it acquires two SPAMM images that are $180^{\circ}$ out of phase prior to subtracting them for estimation of deformations in one direction. For 2D deformation estimation, CSPAMM requires four acquisitions.

In this chapter, a novel tagging pulse sequence, Orthogonal CSPAMM (OCSPAMM), has been proposed which results in the tag lines to be persistent over the entire length of the cardiac cycle while halving the imaging time, when compared to CSPAMM. In comparison to CSPAMM, OCSPAMM eliminates the DC interference of the off-center peaks [44], but does not produce tag patterns in the same direction that are 180 degrees out of phase. The contents of this chapter are based on papers that have been published in [4] and [5].

\section{A. Orthogonal CSPAMM Acquisition}

In this section, a new tagging pulse sequence, Orthogonal CSPAMM (OC- 
SPAMM), is proposed which acquires two perpendicular motion information within the same time as SPAMM while keeping the advantages of CSPAMM.

As seen in Figure 19 in Chapter III, in CSPAMM, two SPAMM tagging sequences 180 degrees out of phase are placed in the same direction, either in frequency encoding or phase encoding direction, resulting in the need for 4 separate acquisitions. In order to obtain the maximum grid amplitude, the tagging pattern $T A G_{A}(x, y)$ and $T A G_{B}(x, y)$ should satisfy the following condition:

$$
T A G_{A}(x, y)+T A G_{B}(x, y)=0
$$

which will produce the $k_{t h}$ tagged image (See Chapter III)

$$
I_{k}=2 Q_{T_{k}} \sin \alpha_{k} e^{-T E / T_{2}^{*}}
$$

In the proposed OCSPAMM sequence, the second SPAMM tag orientation is rotated 90 degrees relative to the first one so that motion information in two directions can be obtained simultaneously. In this case, Equation (63) will not be met any more. This reduces the acquisition time by a factor of two as compared to the traditional CSPAMM, in which two separate imaging sequences are applied per acquisition (See Figure 19). The OCSPAMM sequence timing diagram is shown in Figure 35. As it may be seen in this figure, the first tagging gradient is in the $G_{x}$ direction, while the second tagging gradient is in the $G_{y}$ direction. A TFE-EPI sequence is used to image the modulated magnetization, as shown in Figure 36. TFE-EPI is a fast imaging method using gradient echo. EPI acquires a set of echoes after a single RF excitation with a short phase encoding gradient pulse (a blip) between each echo [14]. A timing diagram of the TFE-EPI sequence is shown in Figure 36.

Visualization of $\mathrm{k}$-space for OCSPAMM sequence is shown in Figure 37. Similar to the CSPAMM sequence of Figure 21, two SPAMM images are subtracted, but unlike the original CSPAMM technique, the second tagged acquisition is orthogonal 


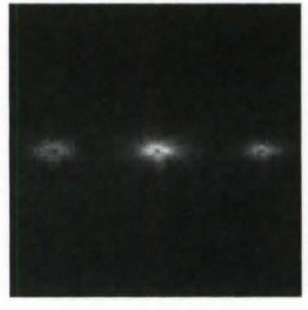

(a)

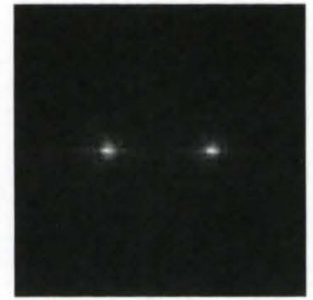

(b)

FIGURE 34-Spectrum of tagged images using (a) SPAMM and (b) CSPAMM.
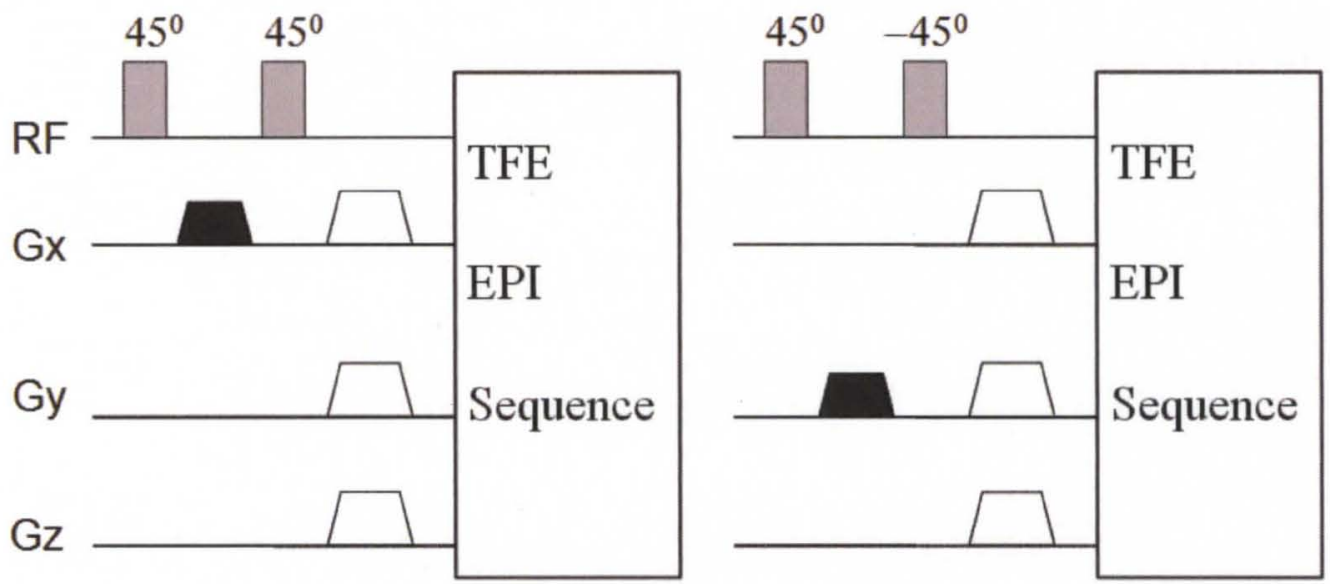

FIGURE 35 - Timing diagram for the OCSPAMM sequence. The first pair of $45^{\circ}$ RF pulses with an interspersed tagging gradient are used to define the tags in $G_{x}$ direction. The second pair of $45^{\circ} \mathrm{RF}$ pulses with an interspersed tagging gradient orthogonal to the first tagging gradient are used to define the tags in $G_{y}$ direction. A TFE-EPI sequence is used for imaging as shown in Figure 36.

to the first one. This approach eliminates the DC component while leaving the offcenter peaks in k-space intact and reduces the acquisition time by a factor of two when compared to the original CSPAMM technique. 


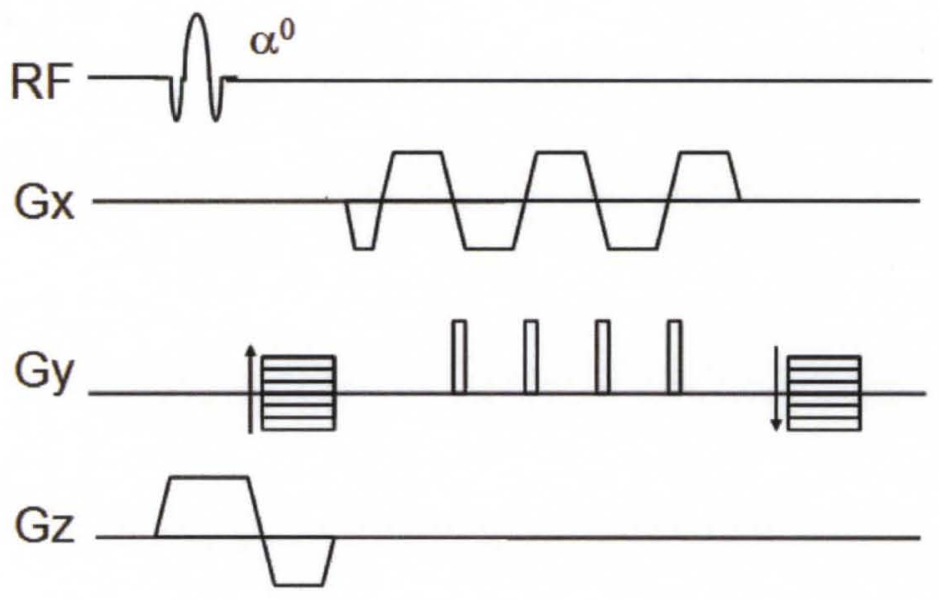

FIGURE 36-A timing diagram of the TFE-EPI sequence used for imaging the modulated magnetization with EPI factor 5. After each RF pulse, five k-space profiles are acquired with the help of the blip gradients in phase encoding direction.

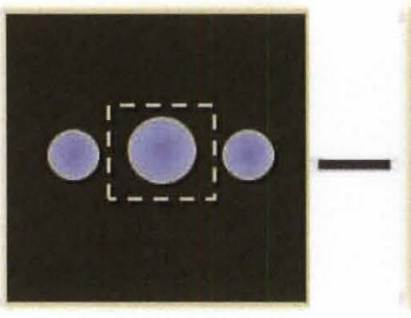

(a)

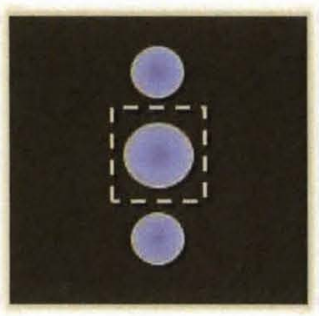

(b)

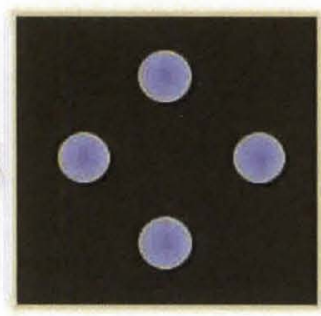

(c)

FIGURE 37-Visualization of k-space for the OCSPAMM sequence. (a) k-space for tagged image with positive tagging pattern (b) k-space for tagged image with negative tagging pattern in orthogonal direction to (a) (c) k-space for OCSPAMM. 


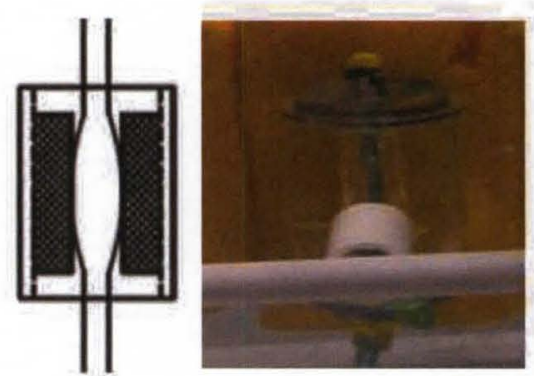

(a)

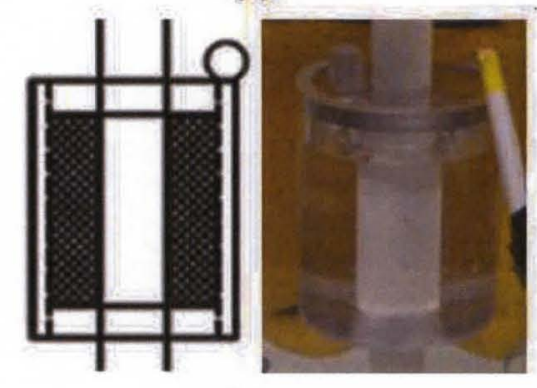

(b)

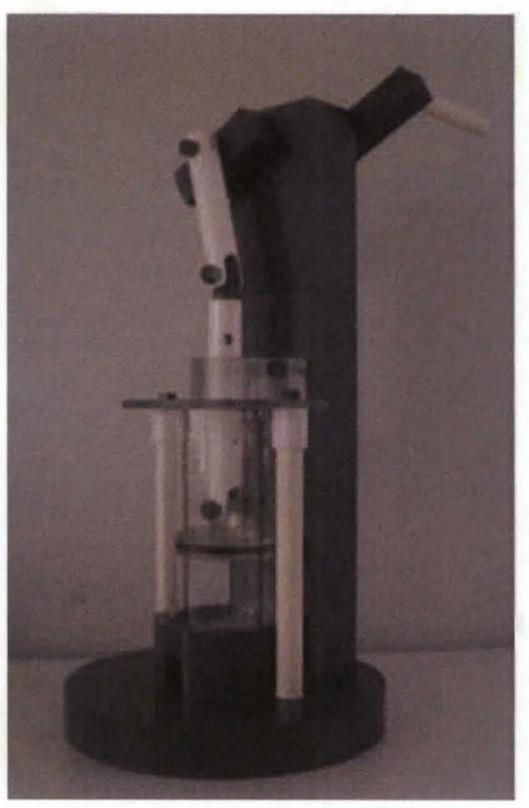

(c)

FIGURE 38 - Phantom components. (a) Contracting cardiac phantom (b) Rotating cardiac phantom (c) Air pump

\section{B. Results}

A cardiac motion phantom, which independently models myocardial wall thickening and rotation in the human heart, was utilized to test the proposed OCSPAMM pulse sequence. The main elements of the LV motion phantom are the air pump, two phantoms within a common enclosure, trigger circuit, and rotation motion actuator. The two phantoms and the air pump are shown in Figure 38. Other than the triggering circuit, all material used in construction of the phantom were non-ferromagnetic and MR compatible (polycarbonate, wood, latex, sponge, di-electric gel) [149].

All imaging experiments were conducted on a 3T Achieva MR scanner (Philips Healthcare, Best, NL) using a two element receive coil. The tag line distance was $7 \mathrm{~mm}$. The phantom was imaged using a turbo gradient echo-echo planar imaging cine pulse sequence (Figure 36) with the following parameters: TR/TE $=9.1 / 4.7 \mathrm{~ms}$, $10^{\circ}$ flip angle, turbo factor 7 , FOV $225 \times 225 \mathrm{~mm}$, in-plane voxel size $2 \times 2 \mathrm{~mm}^{2}$, reconstruction resolution $1.25 \times 1.25 \mathrm{~mm}^{2}$, slice thickness $8 \mathrm{~mm}, 14$ heart phases, 

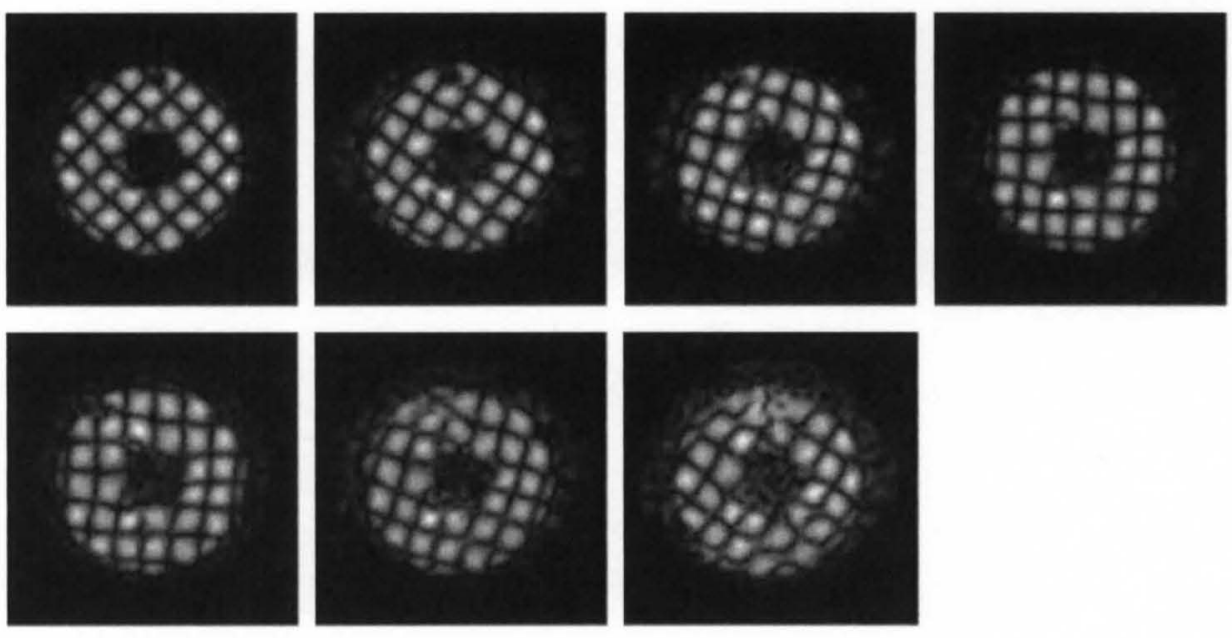

FIGURE 39-Seven Images from fourteen cardiac phases for a rotating phantom using the proposed OCSPAMM pulse sequence. Every other frame during an entire cardiac cycle is shown. The order is from top-left to bottom-right.

$112 \times 85$ acquired matrix, EPI factor 5 . The cardiac cycle was approximately 1000 ms and $T_{1}$ of dielectric gel in the phantom was $728 \mathrm{~ms}$ (similar to myocardial tissue, in-vivo).

Two experiments were conducted on the cardiac phantom to validate the proposed OCSPAMM tagging sequence: one for rotation as shown in Figure 39 and the other for contraction as shown in Figure 41. Seven images from fourteen cardiac phases are displayed. Every other frame during an entire cardiac cycle is chosen. The displaying order is from top-left to bottom-right. The OCSPAMM tagged images showed good image quality and persistent tag contrast for the entire duration of the cardiac cycle. For comparison to SPAMM sequence, Figure 40 shows a cine sequence of SPAMM tagging on the same rotating phantom during one cardiac cycle, where tag fading is clearly evident. Notice that there are some artifacts in the upper and lower portion of the last frame in Figure 41. These were due to susceptibility from the air inside the center of the phantom and are unrelated to the OCSPAMM pulse sequence. 

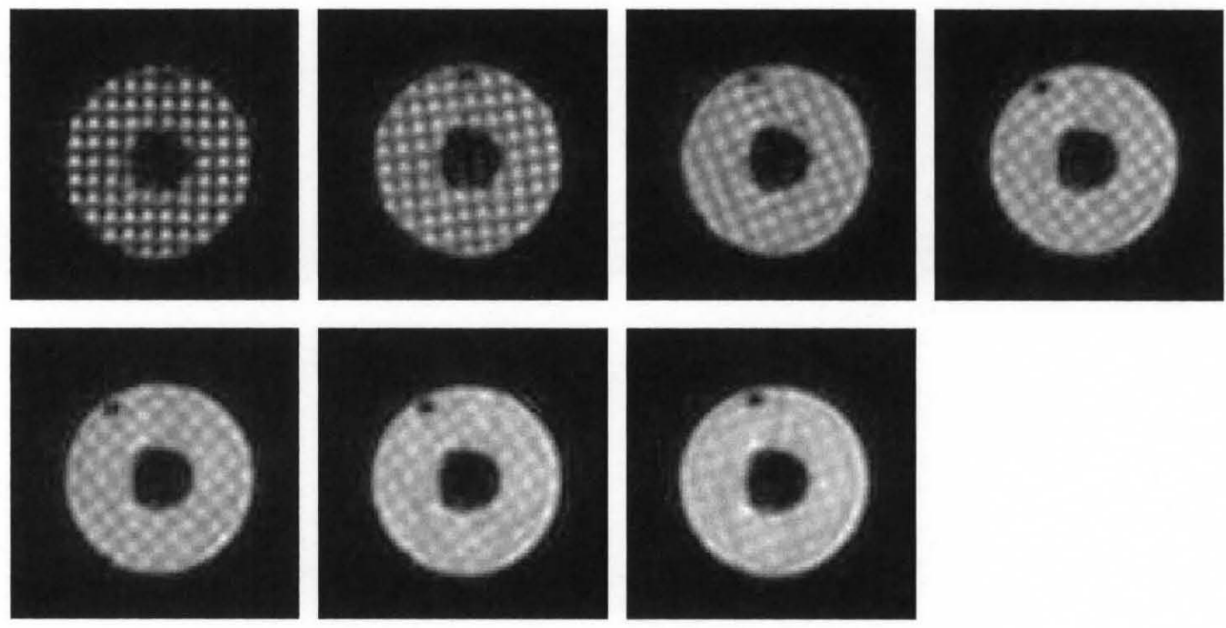

FIGURE 40 -Seven images of fourteen cardiac phases during an entire cardiac cycle for a rotating phantom using SPAMM pulse sequence. Every other frame is shown. The order is from top-left to bottom-right. In comparison to images acquired with OCSPAMM from the same phantom (Figure 39), tag fading is clearly evident.
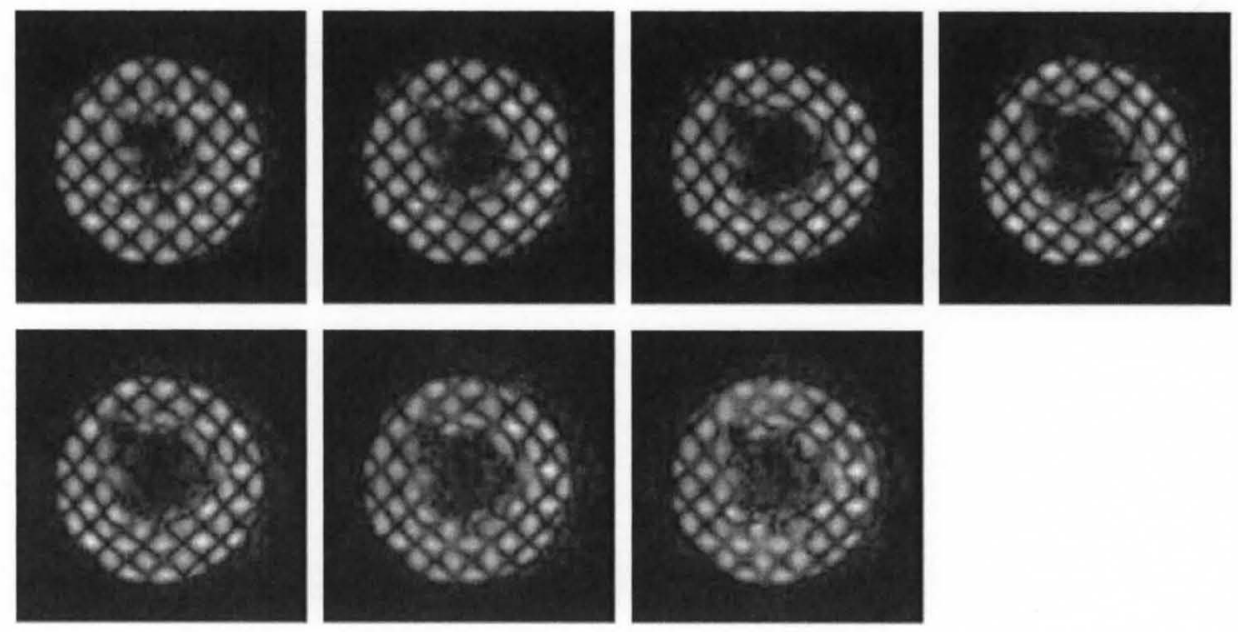

FIGURE 41 -Seven Images from fourteen cardiac phases for a contracting phantom using the proposed OCSPAMM pulse sequence. Every other frame during an entire cardiac cycle is shown. The order is from top-left to bottom-right. 


\section{CHAPTER VII \\ ANALYSIS OF 3D CSPAMM TAGGED MRI DATA WITH 3D SINMOD}

Most of the current motion analysis techniques are applicable to $2 \mathrm{D}+\mathrm{t}$ tagged MR image sequences. 2D motion analysis has its inherent disadvantages, since it can only capture in-plane expansions, contractions, and rotations of the myocardium. However, heart deforms in $3 \mathrm{D}+\mathrm{t}$ with complex twisting motion combined with longitudinal shortening and wall thickening. In this chapter, a novel 3D sine wave modeling (3D SinMod) algorithm is proposed which can accurately recover true 3D cardiac deformations from 3D CSPAMM MRI. An accelerated 3D complementary spatial modulation of magnetization (3D CSPAMM) tagging technique was used to acquire 3D MR data set for the whole-heart in 7 subjects [11]. The entire framework, from data acquisition to data analysis is in $3 \mathrm{D}$ domain, which overcomes the through-plane motion of LV myocardium.

The rest of this chapter is organized as follows: In section VII.A, the data acquisition and preprocessing approach used in the experiments are presented. In section VII.B, the new 3D SinMod technique is proposed and explained. In section VII.C, the computational time is reported. In section VII.D, experimental results using both simulated data and in-vivo 3D+t CSPAMM tagged MRI data from 7 healthy subjects are presented.

\section{A. Data Acquisition}

The data were acquired using the 3D version of the CSPAMM tagging sequence [11].In three breath-holds each with 18 heartbeats, the acquisition was ap- 
plied three times (once for each breath-hold) where in each case, the tagging gradient was rotated in such a way to acquire $3 \mathrm{D}+\mathrm{t}$ data with orthogonal tags. Note that in each acquisition, two 3D SPAMM tagging volumes are acquired and subtracted from each other, which gives CSPAMM data. Typical imaging parameters were: FOV = $108 \times 108 \times 108 \mathrm{~mm}^{3}$, matrix size $=28$ (Frequency Encoding $) \times 14$ (Phase Encoding) $\times 16$ (16 in slice select direction, but only 14 out of 16 were used in reconstruction). In the spatial domain, the image matrix size was $112 \times 112$, slice thickness $=8 \mathrm{~mm}$, and tag spacing was $7 \mathrm{~mm}$. Each data set consisted of $20-24$ frames per cardiac cycle. In this chapter, results of the new deformation analysis approach on 7 in-vivo human data sets are reported.

Figure 42 illustrates the tagging directions and image slice orientations for 3D CSPAMM acquisition. Solid lines represent tagging planes, while dashed lines represent imaging planes. For each cardiac phase, there are three 3D data sets, one tagged in short axis (SA) direction (column a) and two tagged in long axis (LA) directions (columns b and $\mathrm{c}$ ).

Images at select time frames at end-diastole and end-systole are shown in Figure 43 for a single mid-ventricular slice at each orthogonally line tagged 3D CSPAMM data set. The first row is for SA view, and the second and third rows are for two LA views. At end-systole (column 2 in Figure 43), the tag lines contract and rotate (row 1 and row 2) and exhibit longitudinal shortening (row 3). Select slices from the short axis view are shown in Figure 44. The left ventricular contraction and rotation can be observed during systole.

\section{B. 3D Sine Wave Modeling (3D SinMod)}

As with 2D SinMod [9] that was described in Chapter IV, the neighborhood around each voxel in the 3D tagged volume is modeled as a moving sine wavefront 


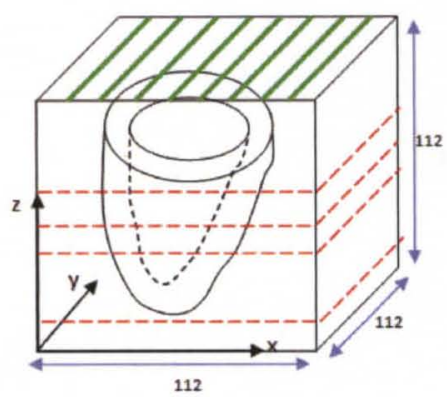

(a)

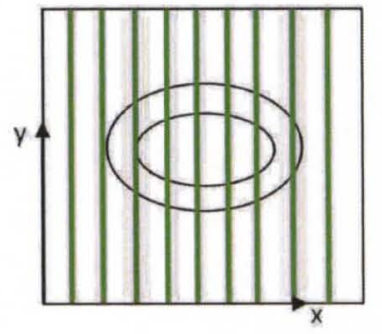

(d)

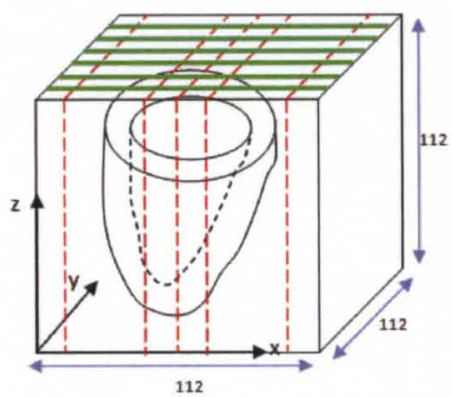

(b)

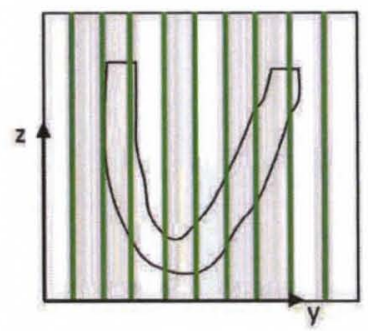

(e)

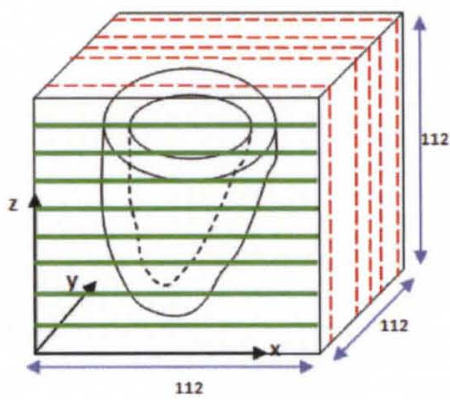

(c)

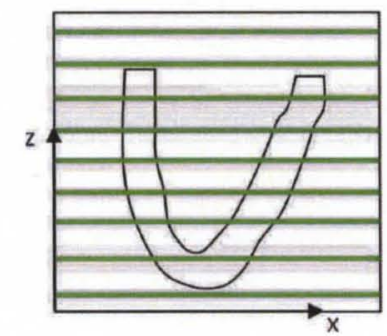

(f)

FIGURE 42 - Illustration of 3D CSPAMM tagging data acquisition for one cardiac phase. Solid lines represent tagging planes, while dashed lines represent imaging planes. (a) Short Axis (SA) stack, (b) First Long Axis (LA1) stack, and (c) Second Long Axis (LA2) stack. (d) A 2D slice from (a). (e) A 2D slice from (b). (f) A 2D slice from (c). 

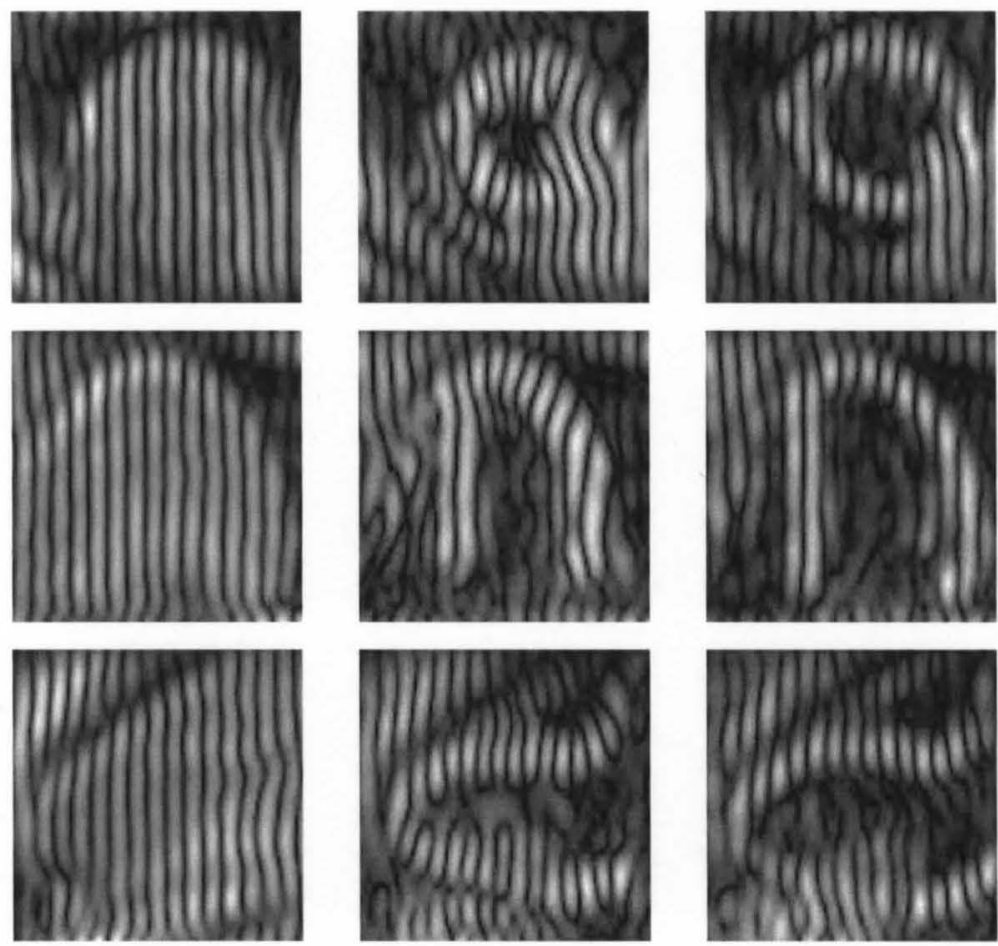

(a)

(b)

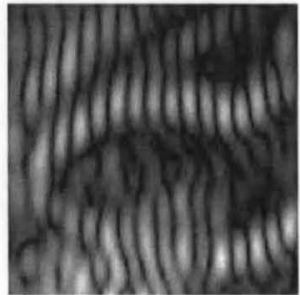

(c)

FIGURE 43 -Example mid-ventricular slices for three data sets with orthogonal tagging directions from 3D CSPAMM sequence. (a) At the beginning of systole. (b) At end-systole. (c) At end-diastole. The first row is from the SA view, and the second and third rows are from two LA views. 

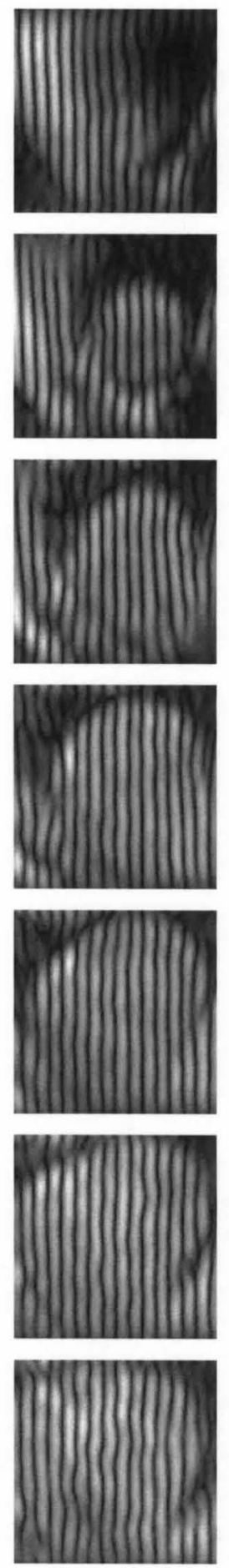

(a)
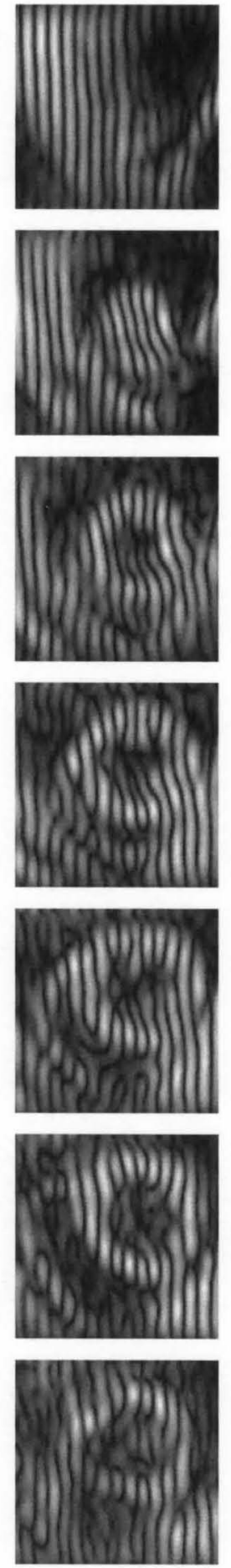

(b)
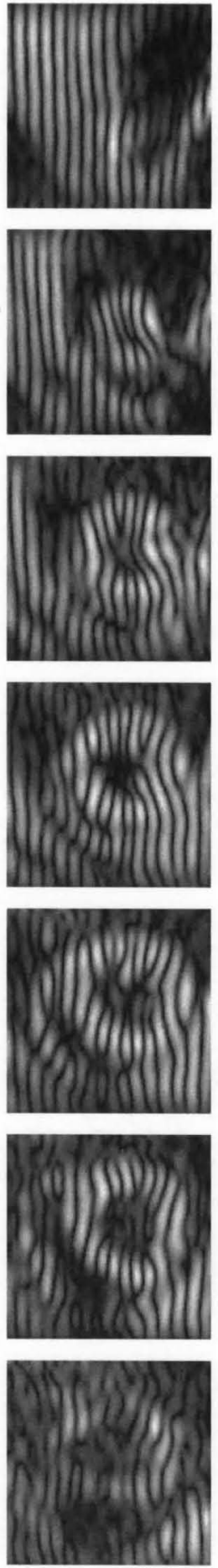

(c)
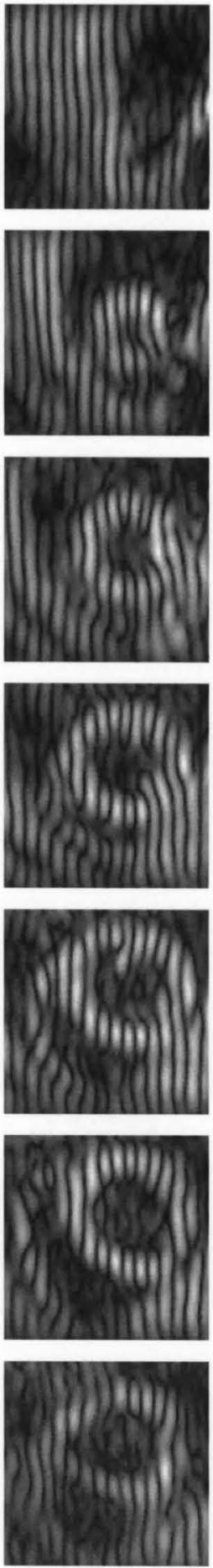

(d)
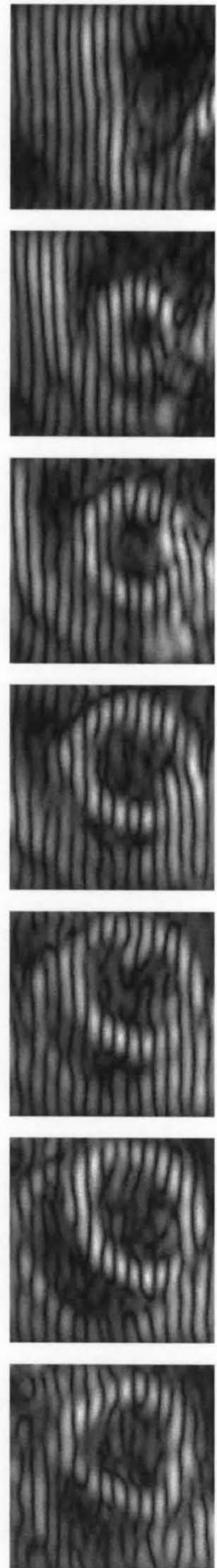

(e)

FIGURE 44-Short axis slices from apex to base acquired with the 3D CSPAMM sequence. (a) The first phase (at the beginning of systole). (b) The 6th phase (at mid-systole). (c) The 11th phase (at end-systole). (d). The 16th phase (at middiastole). (e) The 20th phase (at end-diastole). Row 1: slice 1 (apex). Row 2: slice 3. Row 3: slice 5. Row 4: slice 7. Row 5: slice 9. Row 6: slice 11. Row 7: slice 13 (basal). 
with local frequency and amplitude.

$$
\begin{aligned}
& V_{1}(x, y, z)=A_{1} \cos \left(\omega_{x}\left(x+\frac{u}{2}\right)+\varphi\right)+n_{1}(x, y, z) \\
& V_{2}(x, y, z)=A_{2} \cos \left(\omega_{x}\left(x-\frac{u}{2}\right)+\varphi\right)+n_{2}(x, y, z)
\end{aligned}
$$

where $\omega_{x}$ and $\varphi$ are the spatial frequency and phase of the wave, respectively. $A_{1}$ and $A_{2}$ are wave magnitudes for a 3D volume $V_{1}$ and a short time later, a 3D volume $V_{2}$, while $n_{1}$ and $n_{2}$ are additive noise. $u$ is the displacement between these two volumes at position $(x, y, z)$ along the $x$ direction. The displacements $v$ and $w$ in $y$ and $z$ directions have expressions similar to Equation (65). The principle behind 3D SinMod tracking is that both phase and frequency of the moving wave front for each voxel can be determined directly from the frequency analysis and the 3D displacement can be calculated from the quotient of phase difference and local frequency.

The flow chart for the proposed 3D SinMod algorithm is shown in Figure 45. After obtaining the Fourier Transform of the input volumes $V_{1}(x, y, z)$ and $V_{2}(x, y, z)$ (temporal frames at time $n$ and time $n+1$ ), the same 3D band-pass filter is applied to isolate corresponding spectral peaks and to produce a pair of complex volumes in the Fourier domain. The two complex volumes in Fourier domain following band-pass filtering are noted as $V_{b f 1}\left(\omega_{x}, \omega_{y}, \omega_{z}\right)$ and $V_{b f 2}\left(\omega_{x}, \omega_{y}, \omega_{z}\right)$. Applying a low frequency band-pass filter and a high frequency one to both $V_{b f 1}$ and $V_{b f 2}$ followed by an inverse Fourier transform leads to four complex volumes $V_{b f L f 1}(x, y, z), V_{b f H f 1}(x, y, z)$, $V_{b f L f 2}(x, y, z)$, and $V_{b f H f 2}(x, y, z)$. The reasoning behind application of a LPF and a HPF to $V_{b f 1}$ and $V_{b f 2}$ is to determine the local spatial frequency by power spectra. Then the displacement is the local quotient of phase difference and local frequency 
at that position. The power spectra and cross power spectrum are given by:

$$
\begin{aligned}
& P_{L f}(x, y, z)=\left|V_{b f L f 1}\right|^{2}+\left|V_{b f L f 2}\right|^{2} \\
& P_{H f}(x, y, z)=\left|V_{b f H f 1}\right|^{2}+\left|V_{b f H f 2}\right|^{2} \\
& P_{c c}(x, y, z)=V_{b f L f 1} \bar{V}_{b f L f 2}+V_{b f H f 1} \bar{V}_{b f H f 2}
\end{aligned}
$$

where $\bar{V}$ is the complex conjugate of $V$.

The local frequency $\omega_{x}$ and local displacement $u$ can then be estimated from:

$$
\omega_{x}(x, y, z)=\omega_{c} \sqrt{\frac{P_{H f}}{P_{L f}}} \quad u(x, y, z)=\frac{\arg \left(P_{c c}\right)}{\omega_{x}}
$$

where $\omega_{c}$ is the band-pass center-frequency.

In Figure 45, two 3D image volume at time $\mathrm{n}$ and $\mathrm{n}+1$ are Fourier transformed. A 3D bandpass filter is applied to separate the off-center peak. A window around the band-passed frequencies is inverse Fourier transformed to obtain down-sampled complex volume J. Further analysis produces a down-sampled map of displacement u. This map is upsampled to the initial size of the volume. Repeating the same algorithm for the two LA directions recovers full 3D displacements.

To isolate the spectral peaks, a Butterworth filter was applied in k-space. As shown in Equation (68), the Butterworth filter is a discrete approximation to the Gaussian. In the implementation, a $5^{\text {th }}$ order Butterworth bandpass filter was used with the cutoff frequency half the center frequency.

$$
H=\frac{1}{1.0+\left(\left(\omega-\omega_{c}\right) / \omega_{\text {cutoff }}\right)^{2 n}}
$$

$\omega_{c}$ is the off-center frequency for tagged images, $\omega_{\text {cutoff }}$ is the cutoff frequency, $n$ is the order for Butterworth filter ( $n=5$ was chosen). The filter transfer function is displayed in Figure 46. 


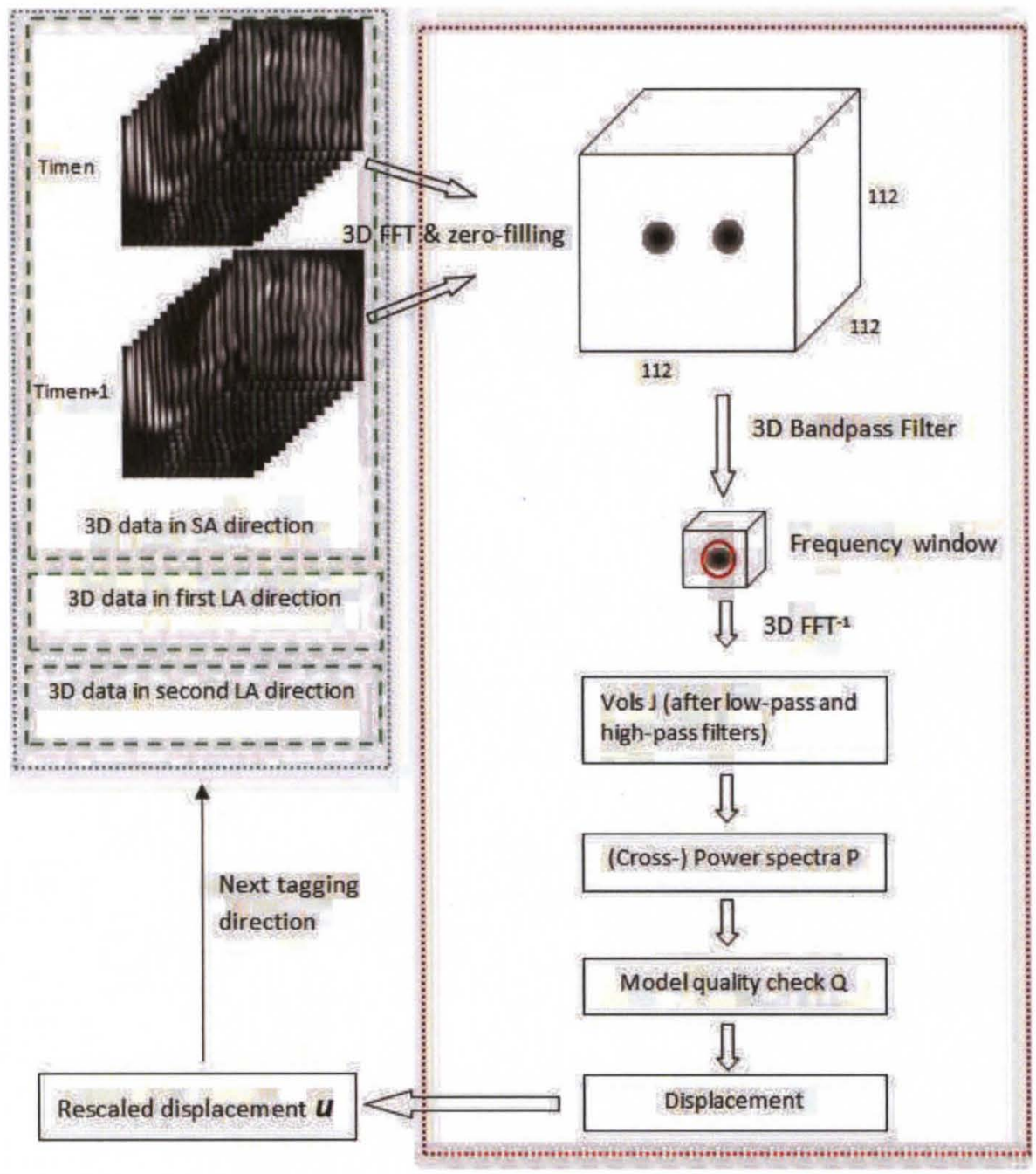

FIGURE 45 - Flow chart of the 3D SinMod algorithm to obtain 3D displacements. Two stacks of 3D image volumes at times $n$ and $n+1$ are Fourier transformed. A 3D bandpass filter is applied to separate one off-center spectral peak. A window around the band-passed frequencies is inverse Fourier transformed to obtain down-sampled complex volume J. Further analysis renders a down-sampled map of displacement $\mu$. This map is upsampled to the initial size of the volume. Repeating the same algorithms for the two LA directions recovers full 3D displacements. 


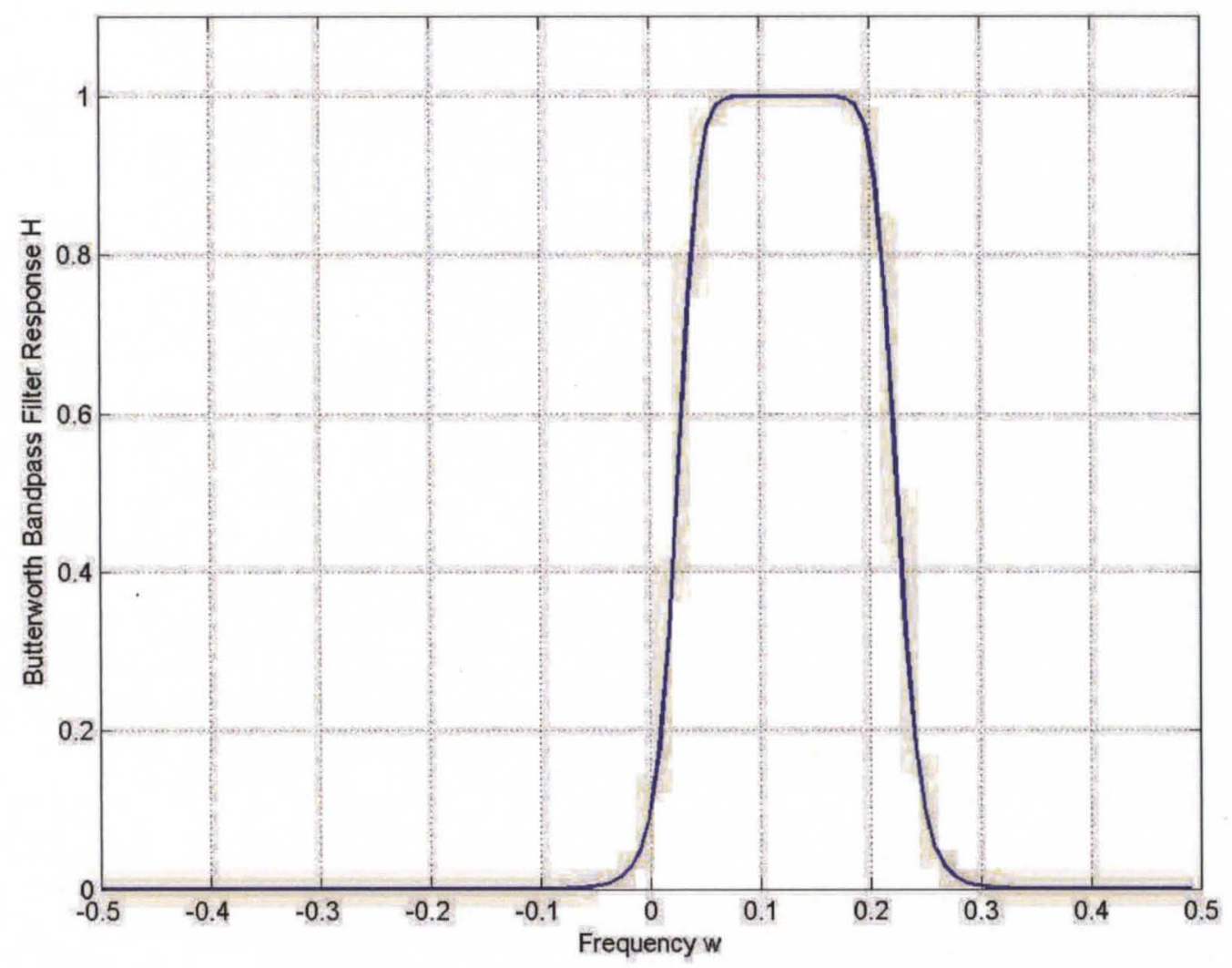

FIGURE 46 -Illustration of Butterworth bandpass filter transfer function in 1D. In this case, cutoff frequency $\omega_{\text {cutoff }}=0.1, \omega_{c}=0.125$, and $n=5$. 


\section{TABLE 6}

Computational time for 3D SinMod algorithm on 7 in-vivo data sets. After zero-fill, each data set consisted of $20-24$ volumetric frames each with 112 voxels.

\begin{tabular}{|l|l|l|}
\hline Data Set Number & $\begin{array}{l}\text { Average Time (sec/min) to } \\
\text { process all 4-D data }\end{array}$ & Average Time/phase (sec) \\
\hline 1 & $358.16 / 5.97$ & 17.91 \\
\hline 2 & $345.71 / 5.76$ & 17.29 \\
\hline 3 & $414.24 / 6.90$ & 17.26 \\
\hline 4 & $409.96 / 6.83$ & 17.82 \\
\hline 5 & $355.71 / 5.93$ & 16.94 \\
\hline 6 & $368.27 / 6.14$ & 17.54 \\
\hline 7 & $353.82 / 5.90$ & 16.85 \\
\hline
\end{tabular}

\section{Computational Time}

All algorithms are programmed in MATLAB (www.mathworks.com) on a conventional $\mathrm{PC}$ (Intel Core i5 $2.6 \mathrm{GHz}$ CPU/4 GB main memory, 64-bit Window 7 operating system). The overall processing time for 3D SinMod processing is about 57 minutes for one $3 \mathrm{D}+\mathrm{t}$ data set (consisting $20-24$ volumetric frames). The average CPU time for calculating the 3D motion fields between a pair of 3D volumes for each of the data sets was 17.37 seconds. Table 6 shows the average computational time for each $4 \mathrm{D}$ data set as well as the average time for calculating 3D displacements between a pair of $3 \mathrm{D}$ volumes. It should be possible to further reduce the computational time by using a faster computer/workstation with implementation in $\mathrm{C}++$.

\section{Validations}




\section{TABLE 7}

The average error between calculated motion fields and ground truth for simulated sequences with Gaussian noise added to the simulated data with standard deviation 0.05 . The reported errors are in pixels.

\begin{tabular}{|l|l|l|l|l|}
\hline & Translation Y & Translation X,Y,Z & Contraction & Expansion \\
\hline AveError & 0.0043 & 0.0329 & 0.0067 & 0.0079 \\
\hline
\end{tabular}

\section{Simulated Motion Fields}

In order to assess the accuracy of the tracking results, $3 \mathrm{D}+\mathrm{t}$ CSPAMM data with the same number of slices and spatial resolution as that of the real data was simulated. Three image sequences each containing 2 volumetric frames but with different displacement fields were generated. The first sequence (Figure 47 (a)) contains tags with 2 pixel uniform translation in the y direction alone. The second sequence (Figure 47 (b)) contains tags with 2,3, and 2 pixel gradual translations in $\mathrm{x}, \mathrm{y}$, and $\mathrm{z}$ directions. Non rigid motion, such as contraction (Figure 47 (c)) and expansion were also applied to test the algorithm. The simulated displacement fields for the translating sequence and the contracting sequence are illustrated in Figure 47. Expansion can be considered as the inverse of the contraction motion.

The proposed 3D SinMod algorithm was applied to the artificially deformed data sets, permitting comparison between the algorithm estimated deformations in the presence of additional Gaussian noise and the known true deformations. Tracking error was calculated for every material point within the $3 \mathrm{D}$ volume and then averaged. The performance of the proposed 3D SinMod method, reported here as the average error in pixels, is shown in Table 7. 

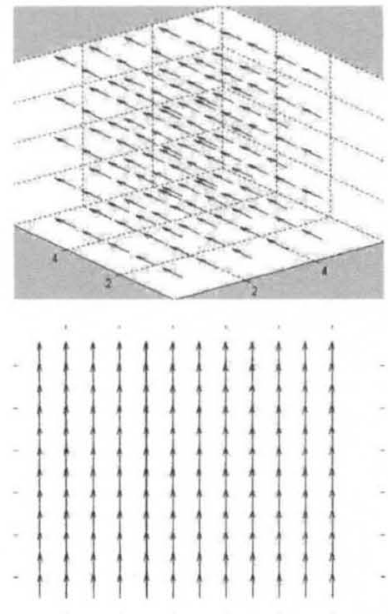

(a)

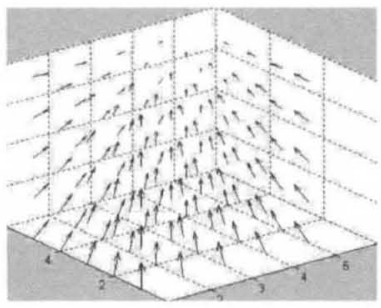

$2 \geq 2: 3 \geq 0$

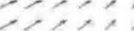

בית

$1: 1,1$

(6),

(b)

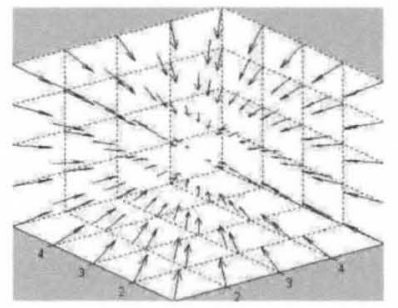

$20+1+1960$

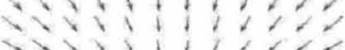

- 2 - 20 :

$\cdots \cdots \cdots$

E

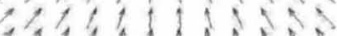

(c)

FIGURE 47 - Displacement fields in the simulated sequence. (a) Translation displacement field in the y direction. (b) Non-homogeneous translations in $\mathrm{x}, \mathrm{y}, \mathrm{z}$ directions. (c) Contraction displacement field. The first row displays 3D visualization, while the second row is the $2 \mathrm{D}$ projection of the first row.

\section{Comparison with 3D HARP}

Seven real 3D+t CSPAMM data sets of healthy human subjects were used to validate the proposed 3D SinMod algorithm. For each data set, there were about $20 \times 3$ image volumes, with 14 slices in each volume. Therefore, in total, there were about $20 \times 3 \times 14=840$ images in each subject data set.

For each image volume in the reference state, ten mid-wall contours, constructed from multiple landmark points with about $5^{\circ}$ separation were defined on different short-axis slices. The reference state was chosen to be the last cardiac phase which had good myocardial-blood pool contrast permitting good contour definition. The proposed 3D SinMod algorithm was applied to every consecutive pair of cardiac phases and the displacement fields were reconstructed. In order to validate the results, the mid-wall contours at the reference state were deformed to the following phases according to the motion fields obtained from 3D SinMod and results were compared with the deformed contours obtained from 3D HARP [11]. Figure 48 shows the work- 


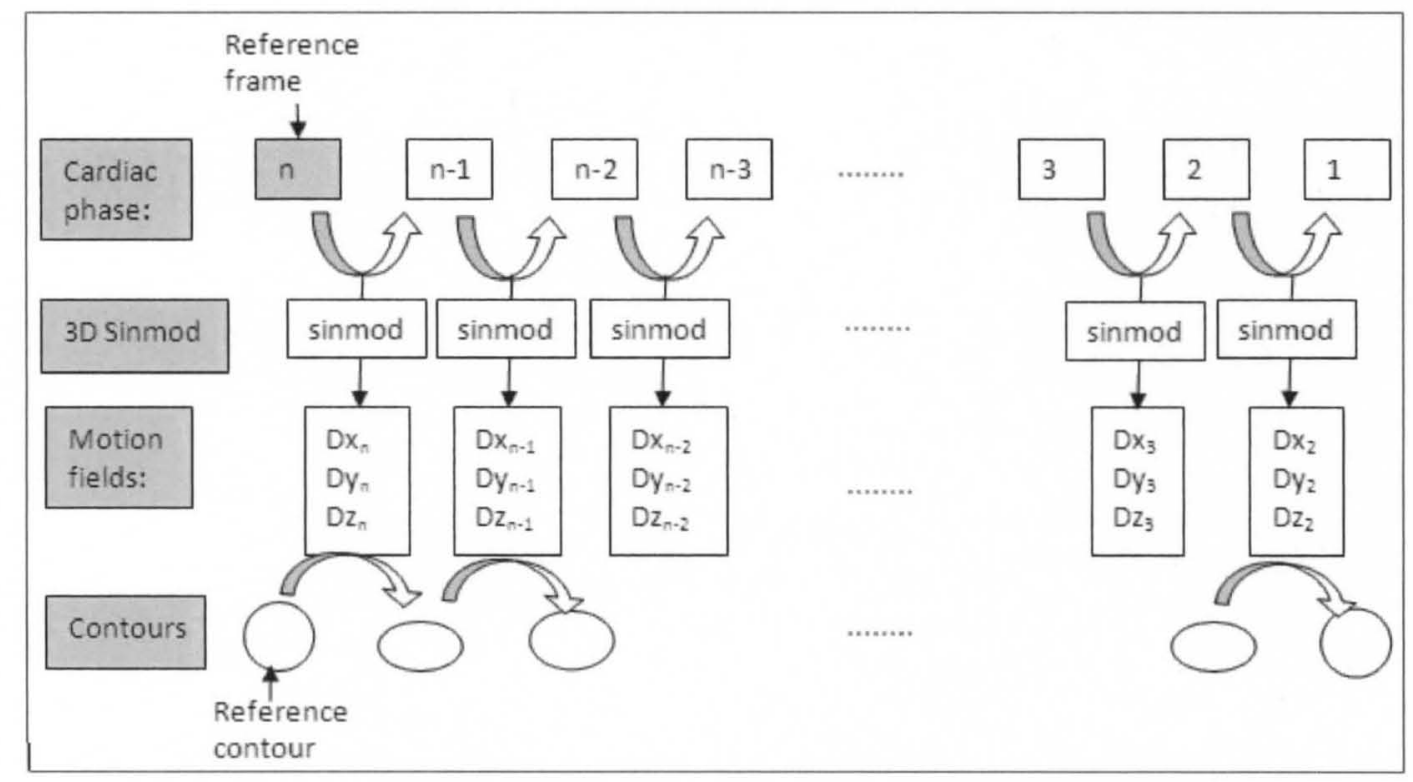

FIGURE 48-Flow diagram for comparison of mid-wall contour deformation using motion field from 3D SinMod algorithm with 3D HARP.

flow for this process. In Figure 49, deformed mid-wall contours from SinMod (in red) and from HARP (in green) at a specific mid-ventricular slice location are displayed. There is a good degree of correspondence between the two. Table 8 shows the average error between deformed mid-wall contours from 3D SinMod and 3D HARP for slices 2 to 10 over all cardiac phases. The reported errors are in pixels.

In Figure 50, mid-wall contours for all slices are shown. Comparing (a) at beginning of systole and (b) at end-systole, longitudinal shortening from base to apex may easily be observed.

\section{Comparison of Warped with Manually Delineated Tag Lines}

All tag lines on 11 slices (from apex to base) and over 11 systolic phases in the same seven 3D CSPAMM tagged data sets were manually delineated. Subsequently, the manually delineated tag lines at reference frame were warped to all the systolic frames and the location of the warped tag lines were compared to location of manually 
TABLE 8

The average error between deformed mid-wall contours from 3D SinMod and 3D HARP for slices 2 to 10 over all cardiac phases. The reported errors are in pixels.

\begin{tabular}{|l|l|l|l|l|l|l|l|}
\hline & data 1 & data 2 & data 3 & data 4 & data 5 & data 6 & data 7 \\
\hline slice 2 & 0.90 & 1.16 & 0.86 & 0.86 & 0.70 & 0.74 & 0.82 \\
\hline slice 3 & 0.79 & 1.36 & 0.68 & 0.99 & 0.59 & 0.87 & 0.81 \\
\hline slice 4 & 0.72 & 1.05 & 0.74 & 0.84 & 0.49 & 0.86 & 0.96 \\
\hline slice 5 & 0.73 & 0.88 & 0.75 & 0.95 & 0.54 & 0.83 & 0.95 \\
\hline slice 6 & 0.72 & 0.92 & 0.67 & 0.78 & 0.67 & 0.87 & 1.05 \\
\hline slice 7 & 0.80 & 1.00 & 0.68 & 0.82 & 0.84 & 0.95 & 1.03 \\
\hline slice 8 & 0.96 & 0.94 & 0.93 & 0.90 & 1.12 & 0.94 & 1.01 \\
\hline slice 9 & 1.28 & 0.97 & 1.24 & 1.22 & 1.36 & 0.94 & 1.37 \\
\hline slice 10 & 1.40 & 1.16 & 1.60 & 1.36 & 1.64 & 1.07 & 1.98 \\
\hline
\end{tabular}



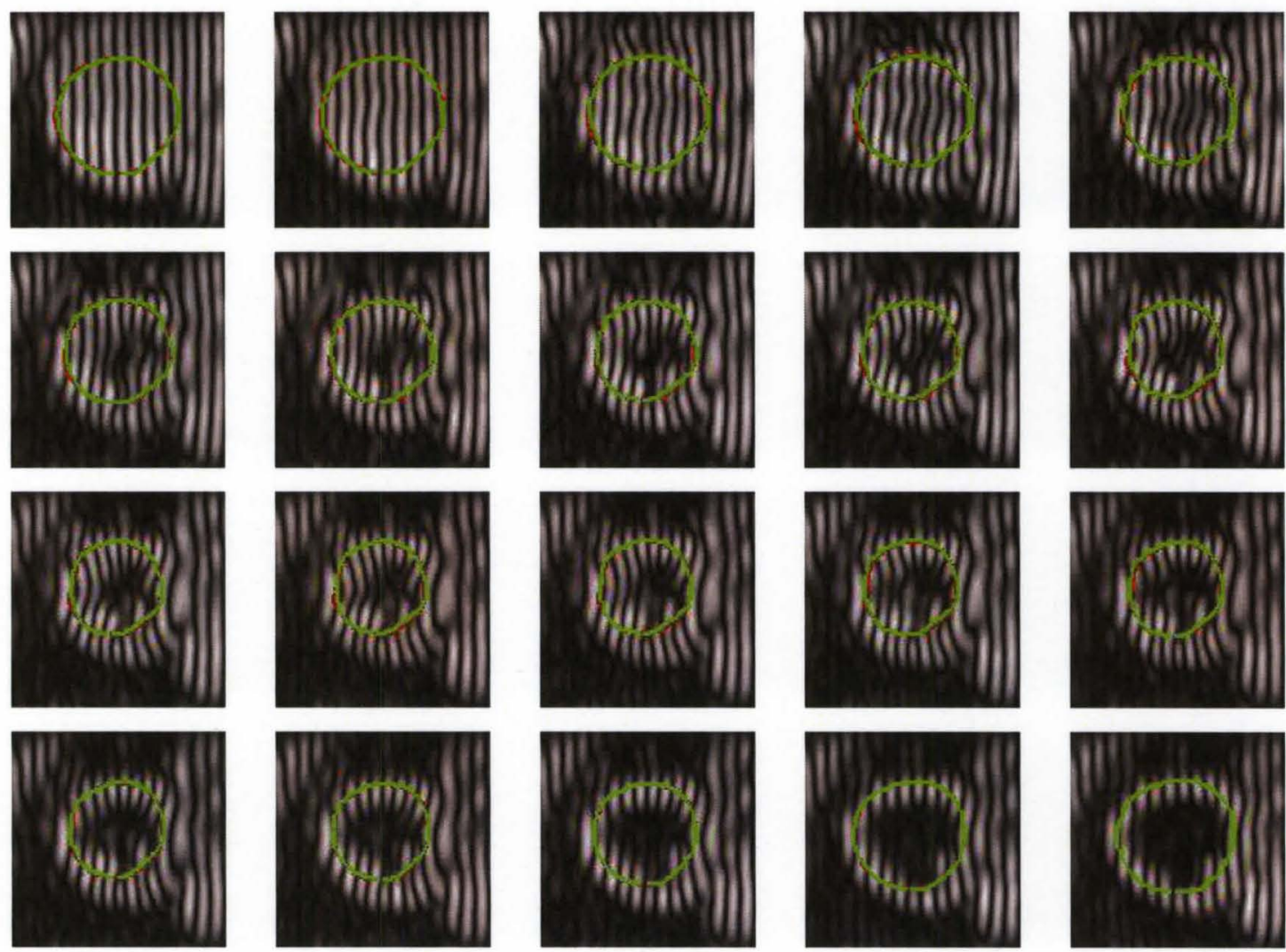

FIGURE 49-Mid-wall contours at a mid-ventricular slice for all phases. Traversing from top-left to the bottom-right are phases 1 to 20. The red contours are deformed with motion field from 3D SinMod. The green contours are deformed with motion field from 3D HARP.

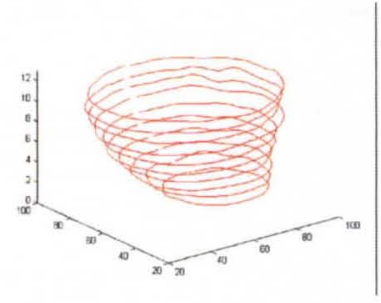

(a)

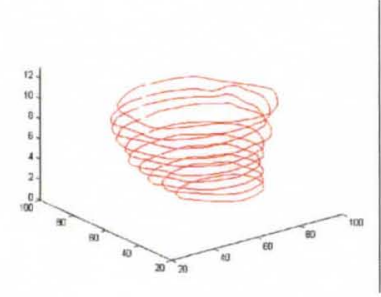

(b)

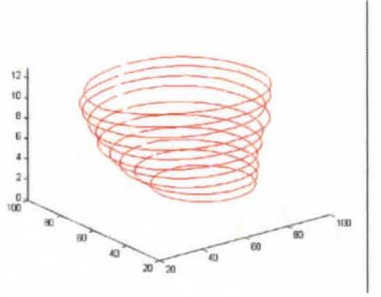

(c)

FIGURE 50-3D mid-wall contours tracked by 3D SinMod. (a) At the beginning of systole. (b) At end-systole. (c) At end-diastole. 


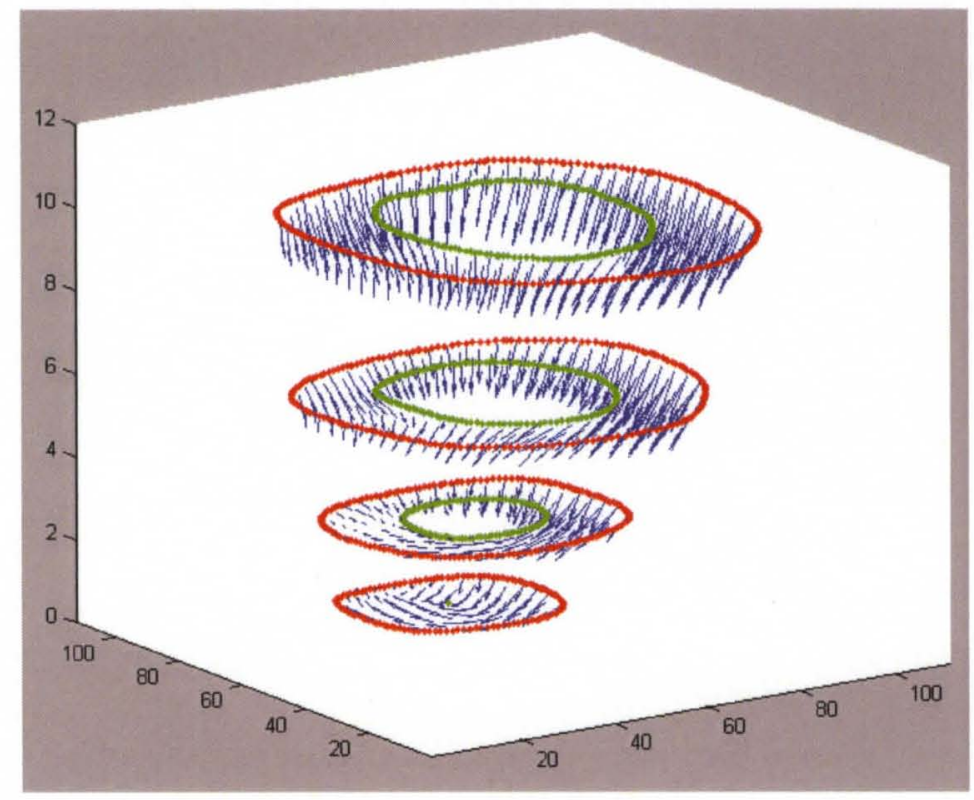

FIGURE 51 - End systolic 3D motion field for slice 1, 3, 6, and 10 for data set 1.

delineated tag lines and an average error for all slices at each phase was computed. Figure 65 displays the average error as a function of time for each of the 7 data sets. As may be observed, all errors are in the sub-pixel range.

\section{E. Results}

\section{Visualization of Motion Fields}

Left ventricular endocardial and epicardial contours were traced manually at all phases for all 3D data sets. The calculated 3D motion fields at apex, apical, midcavity, and basal slices are shown in Figure 51 to Figure 57 for data sets 1 to 7 . The projected 2D end-systolic motion fields on selected slices for each data set are shown in Figures 58 to 64. The selected slices correspond to apical, mid-cavity, and basal locations. 


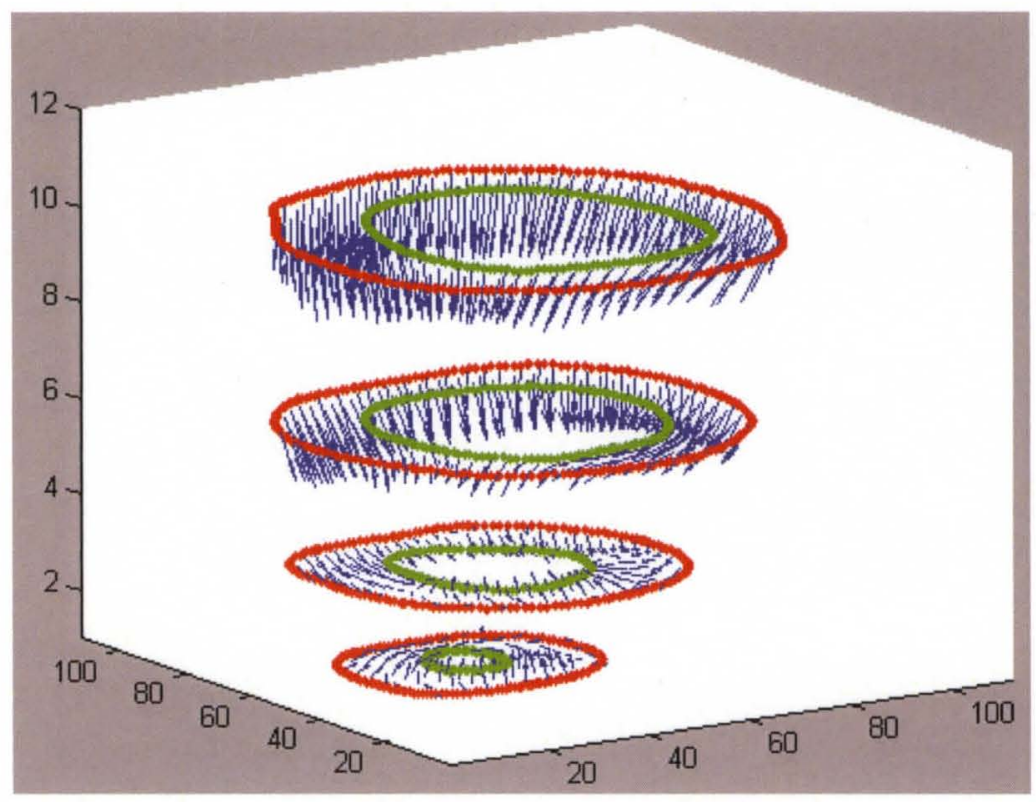

FIGURE 52 - End systolic 3D motion field for slice 1, 3, 6, and 10 for data set 2 .

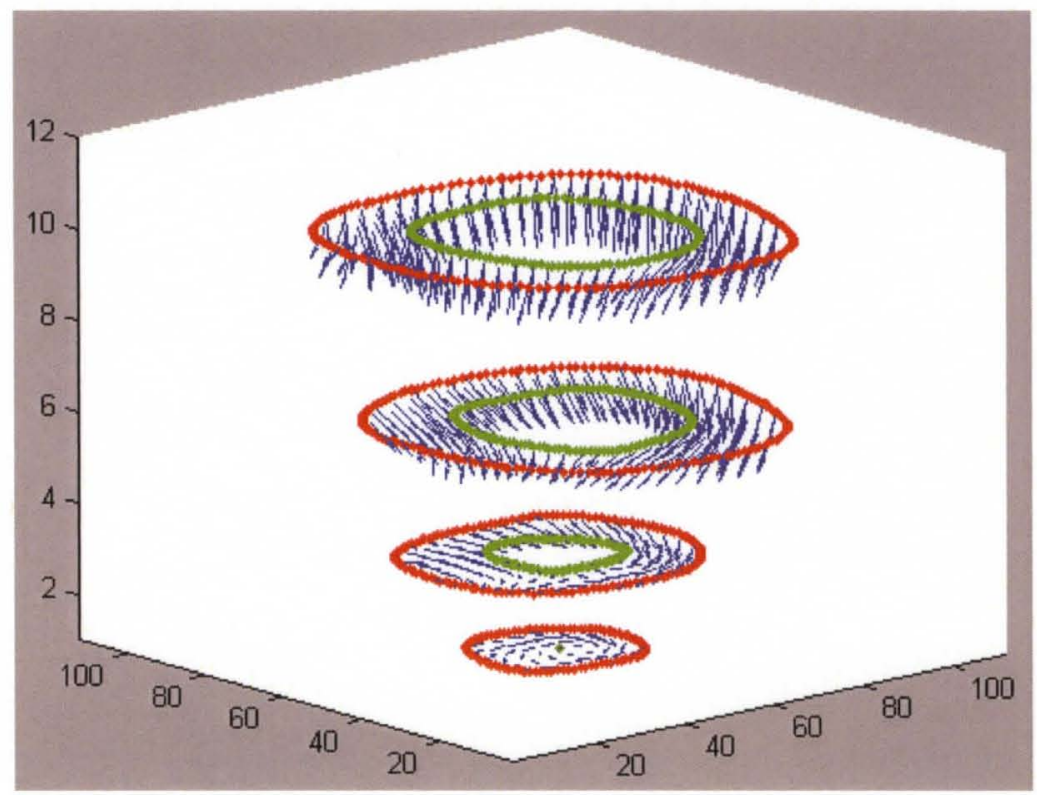

FIGURE 53 - End systolic 3D motion field for slice 1, 3, 6, and 10 for data set 3 . 


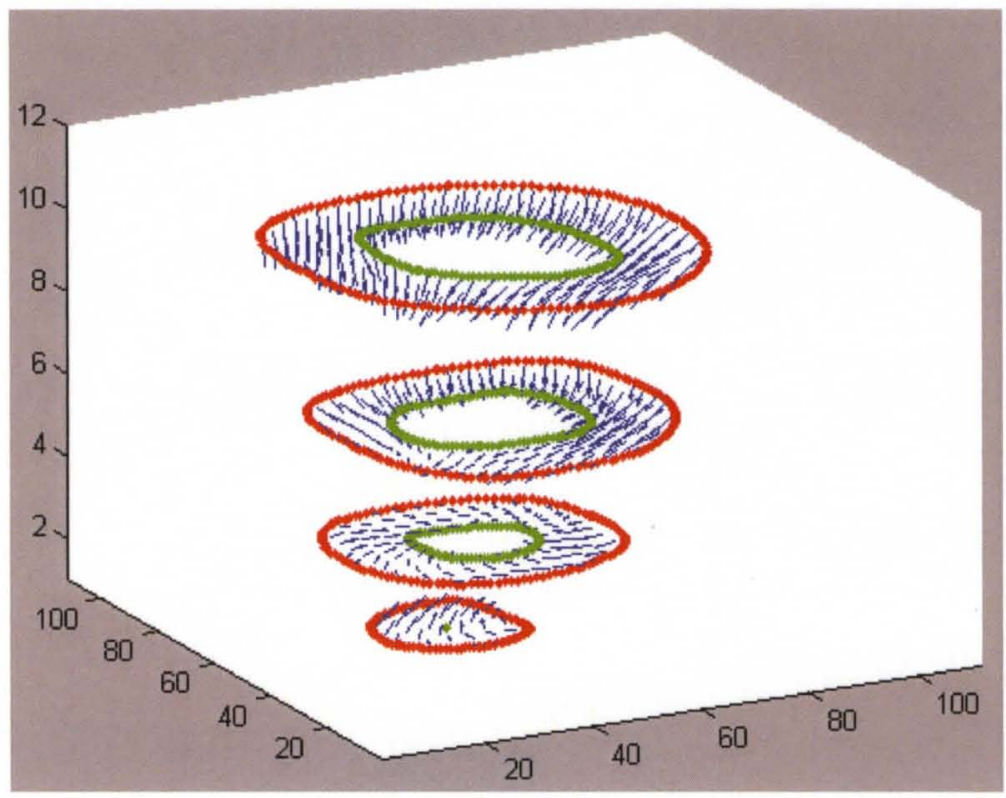

FIGURE 54-End systolic 3D motion field for slice 1, 3, 6, and 10 for data set 4 .

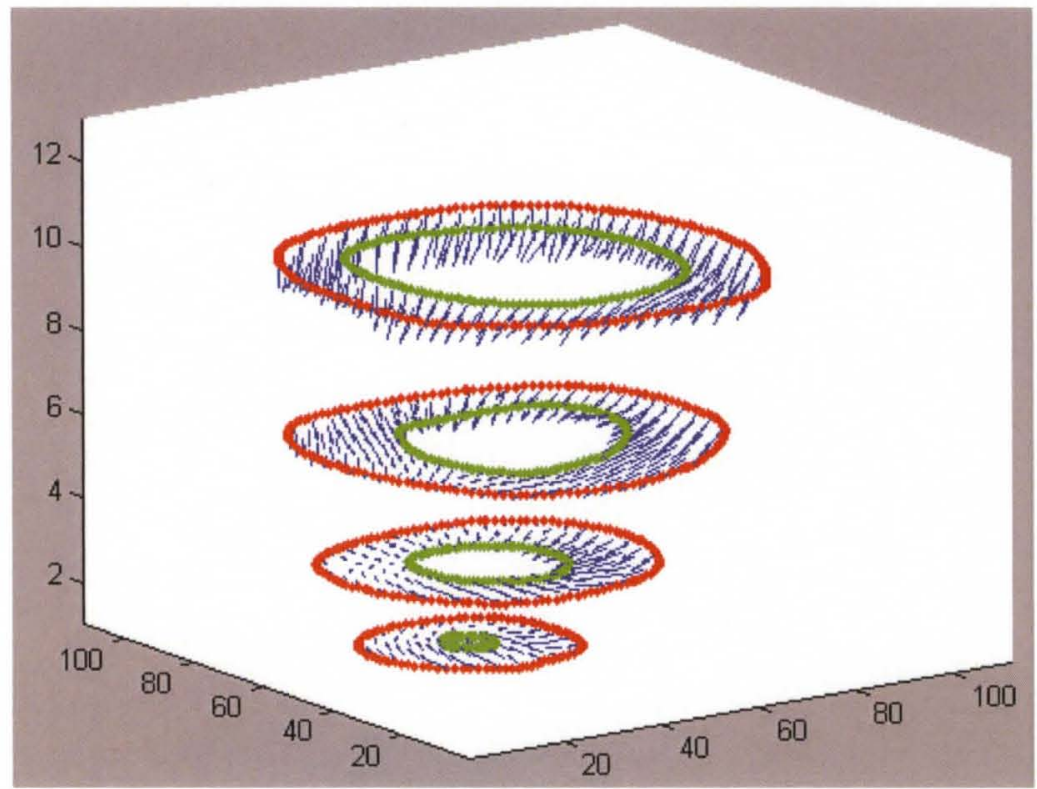

FIGURE 55-End systolic 3D motion field for slice 1, 3, 6, and 10 for data set 5 . 


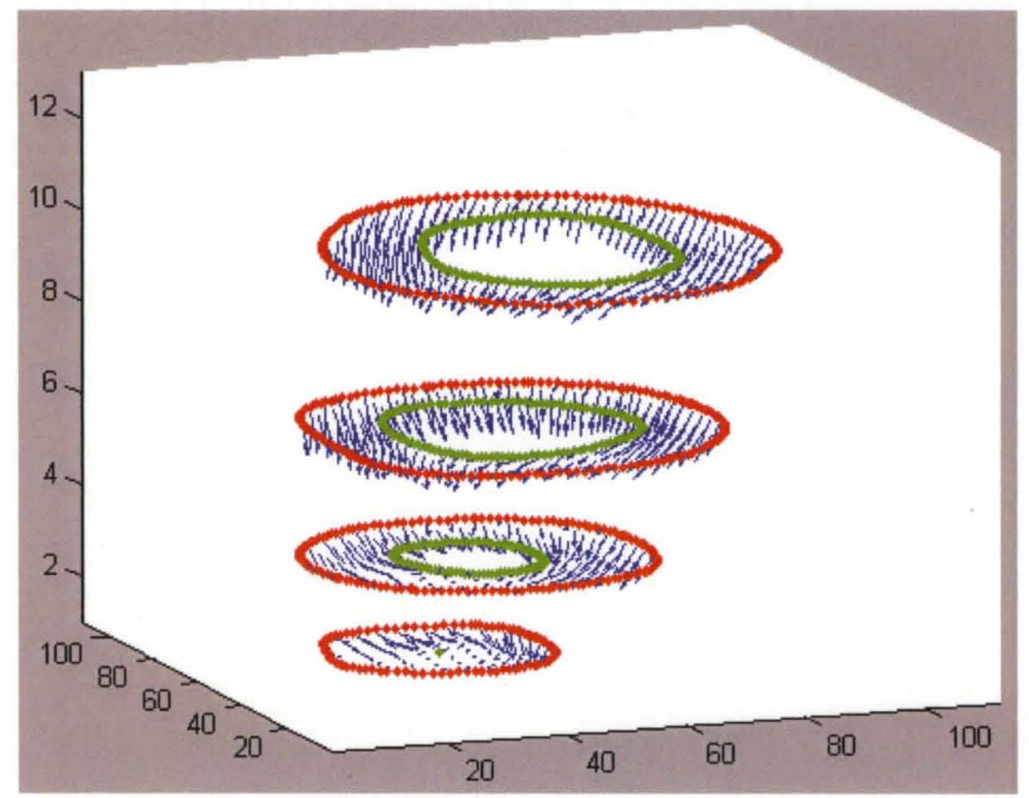

FIGURE 56-End systolic 3D motion field for slice 1, 3, 6, and 10 for data set 6 .

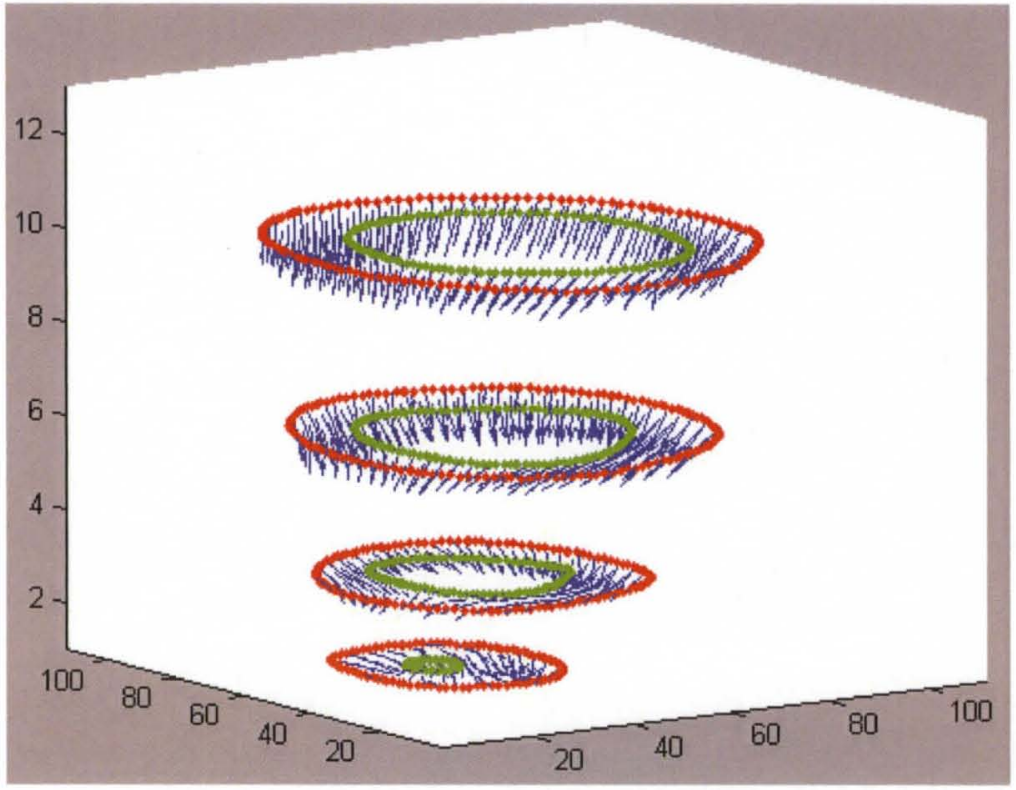

FIGURE 57 -End systolic 3D motion field for slice 1, 3, 6, and 10 for data set 7 . 

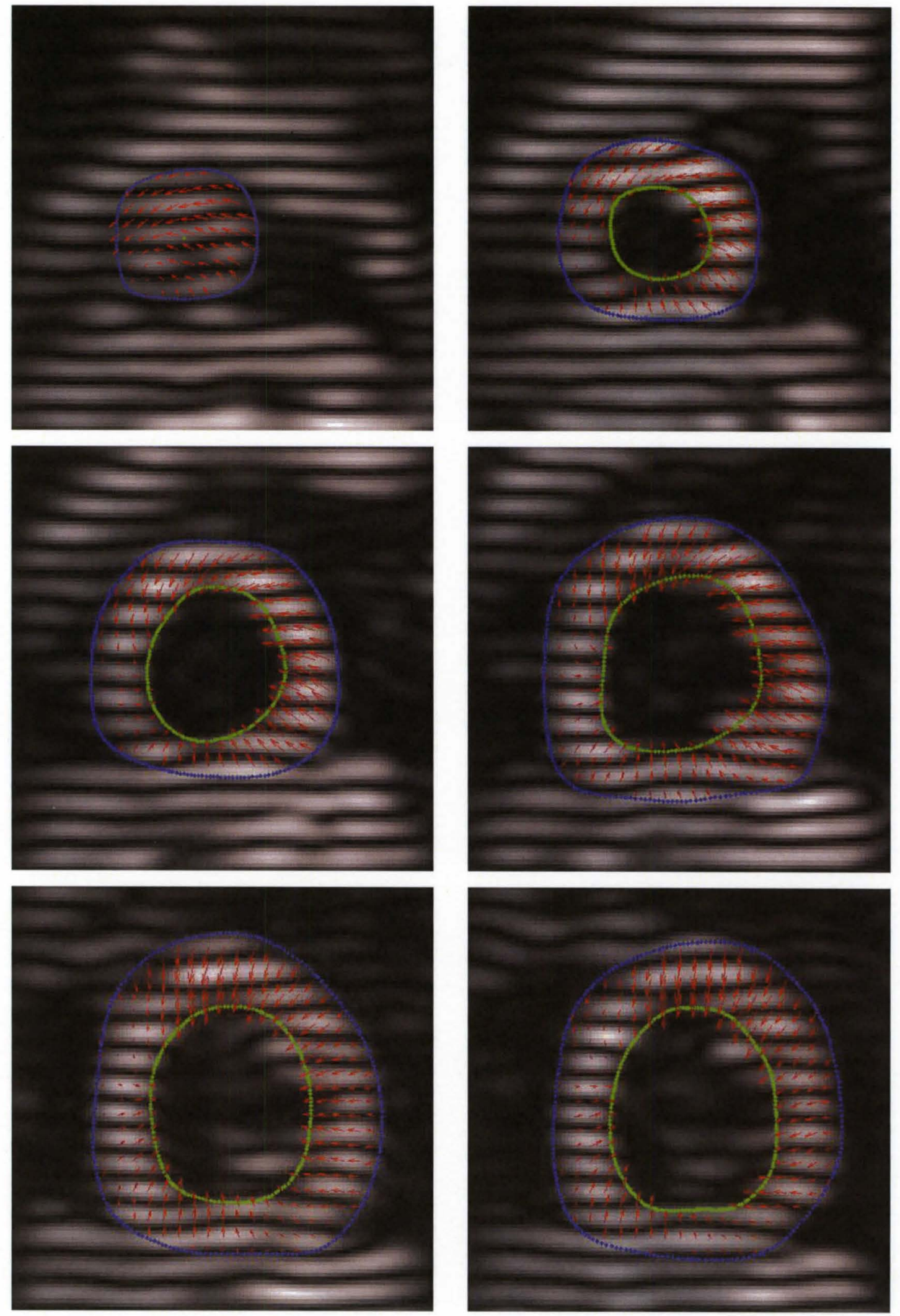

FIGURE 58-End systolic motion field on slices 1, 3, 5, 7, 9, 10 for data set 1 (row 1: apex, row 2: mid-ventricular, row 3: base). 

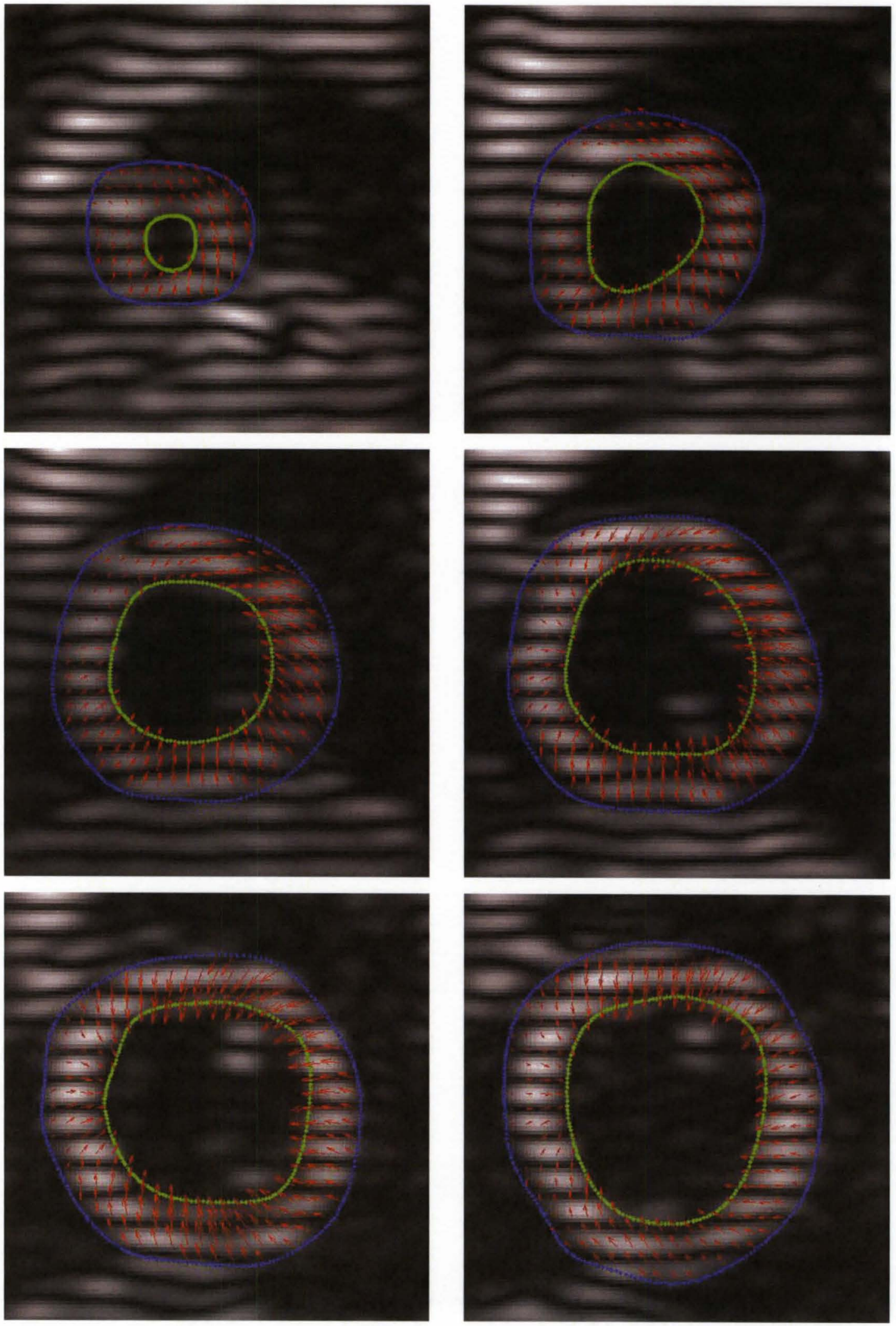

FIGURE 59-End systolic motion field on slices 1, 3, 5, 7, 9, 10 for data set 2 (row 1: apex, row 2: mid-ventricular, row 3: base). 

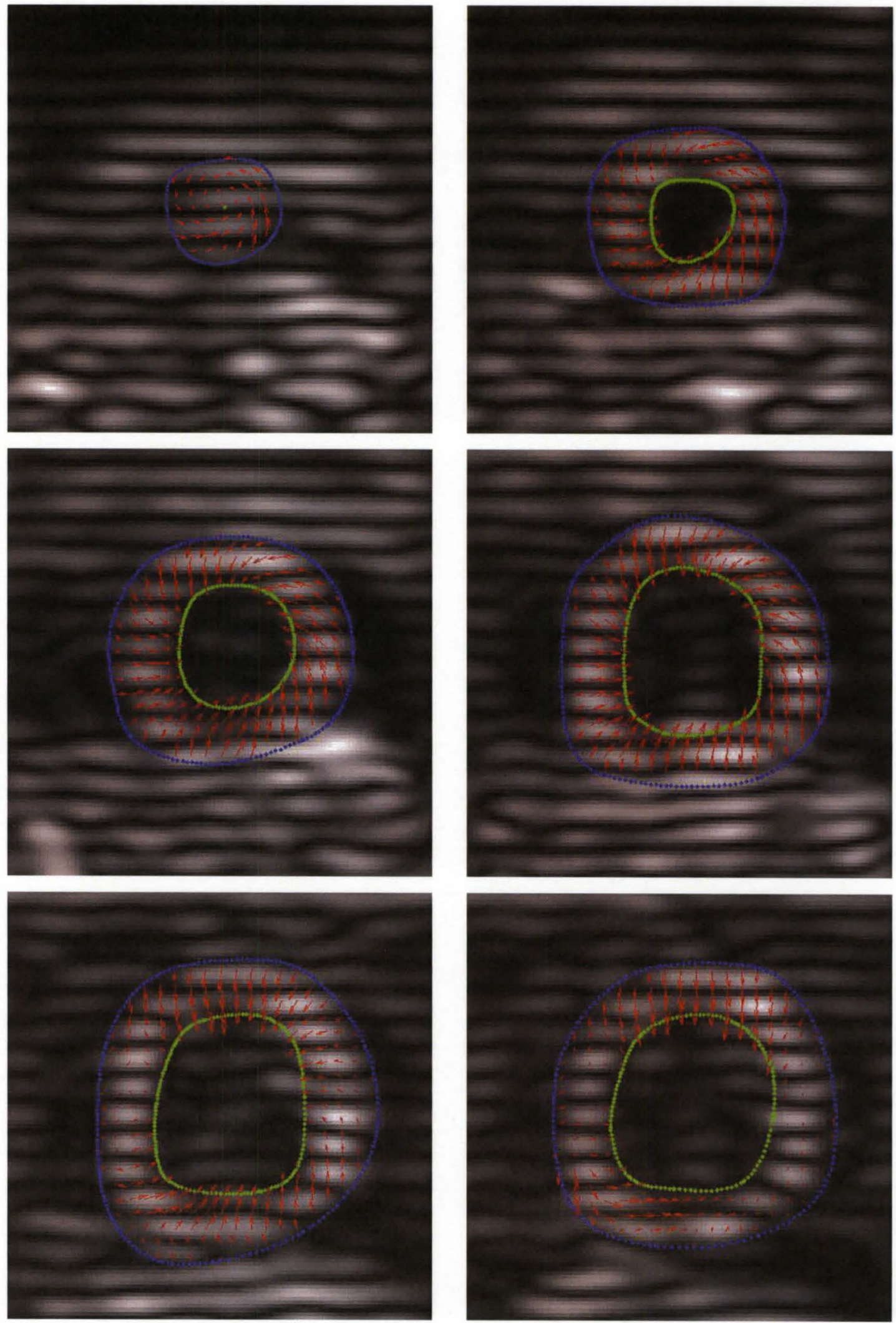

FIGURE 60-End systolic motion field on slices 1, 3, 5, 7, 9, 10 for data set 3 (row 1: apex, row 2: mid-ventricular, row 3: base). 

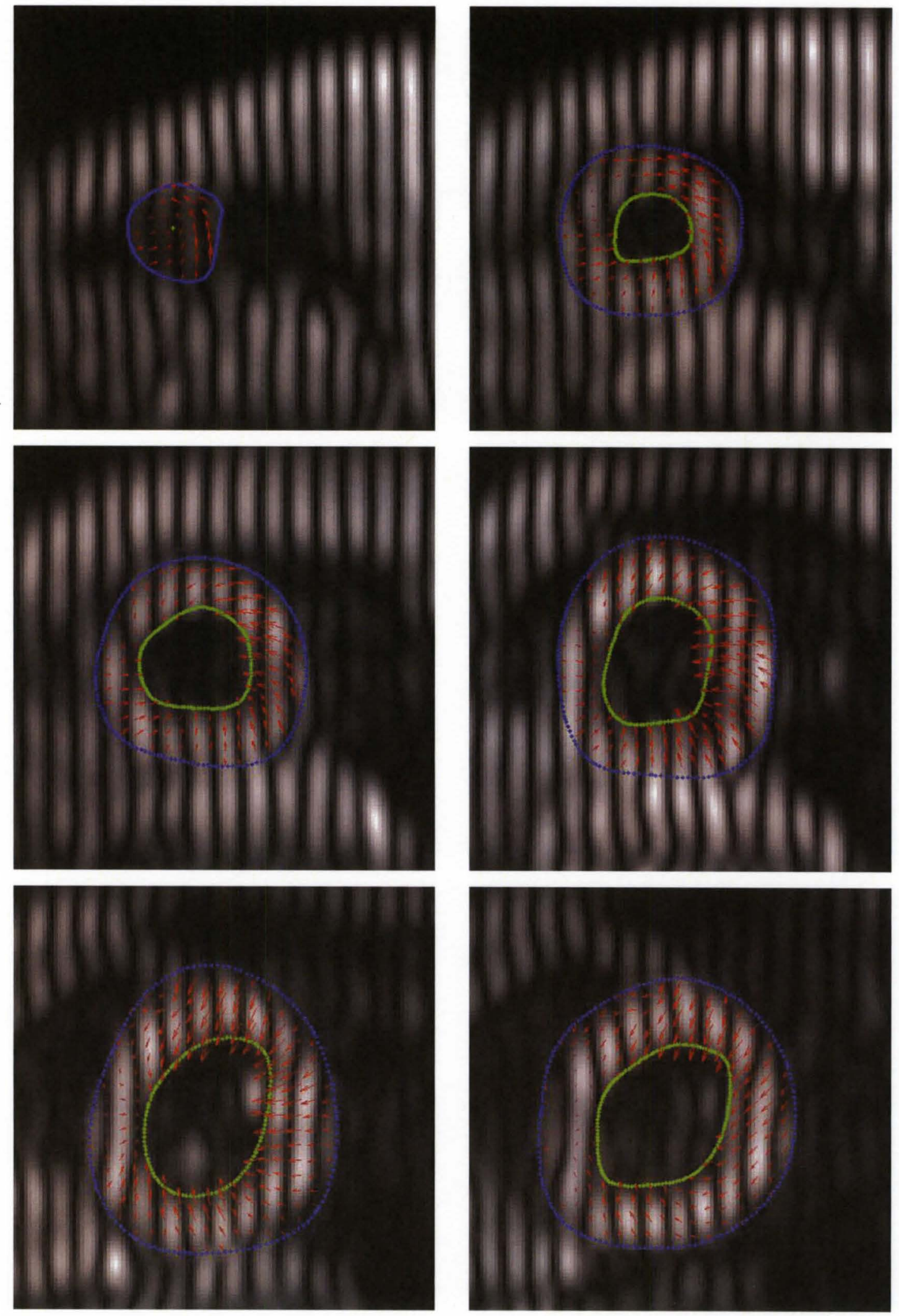

FIGURE 61 -End systolic motion field on slices 1, 3, 5, 7, 9, 10 for data set 4 (row 1: apex, row 2: mid-ventricular, row 3: base). 

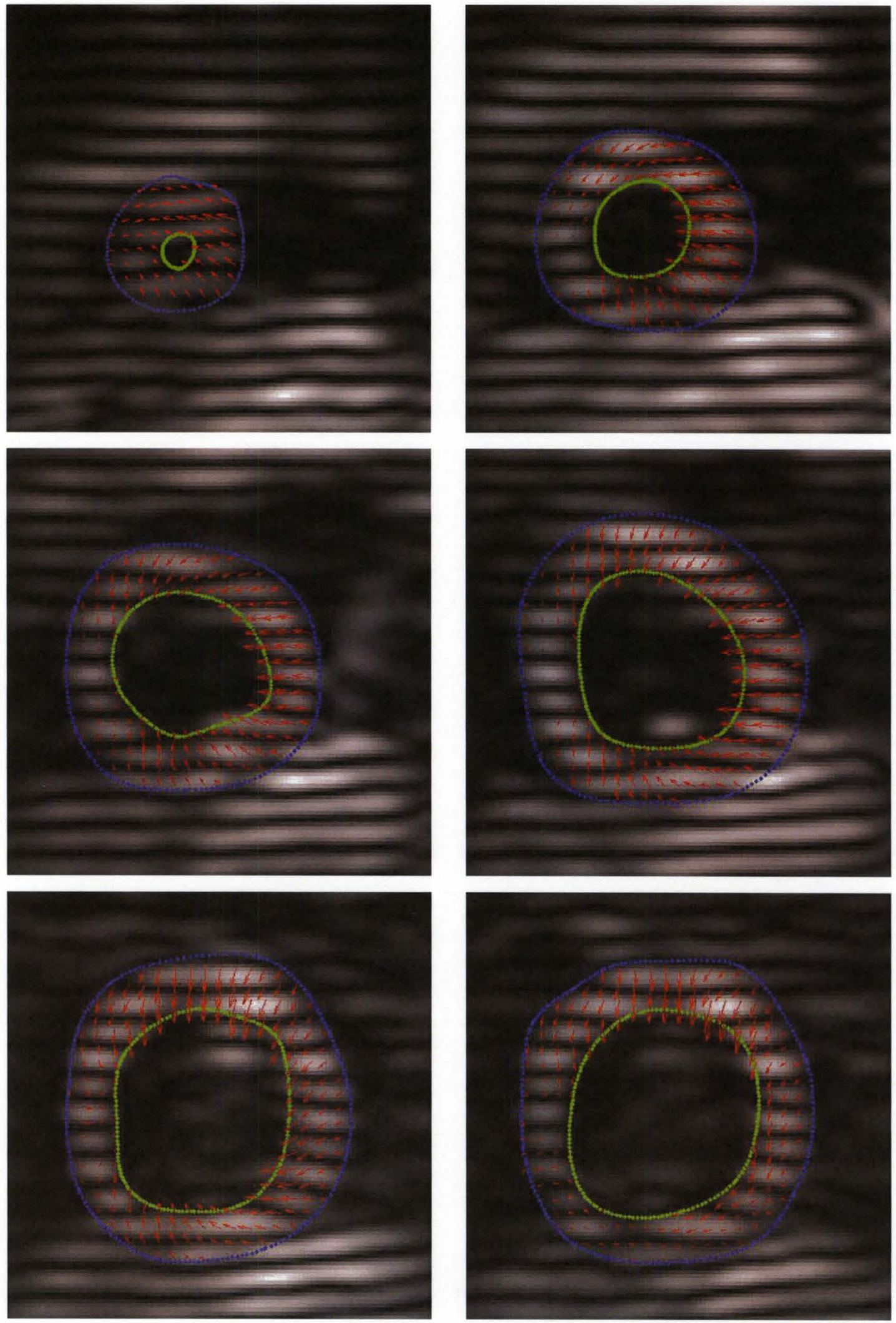

FIGURE 62 -End systolic motion field on slices 1, 3, 5, 7, 9, 10 for data set 5 (row 1: apex, row 2: mid-ventricular, row 3: base). 

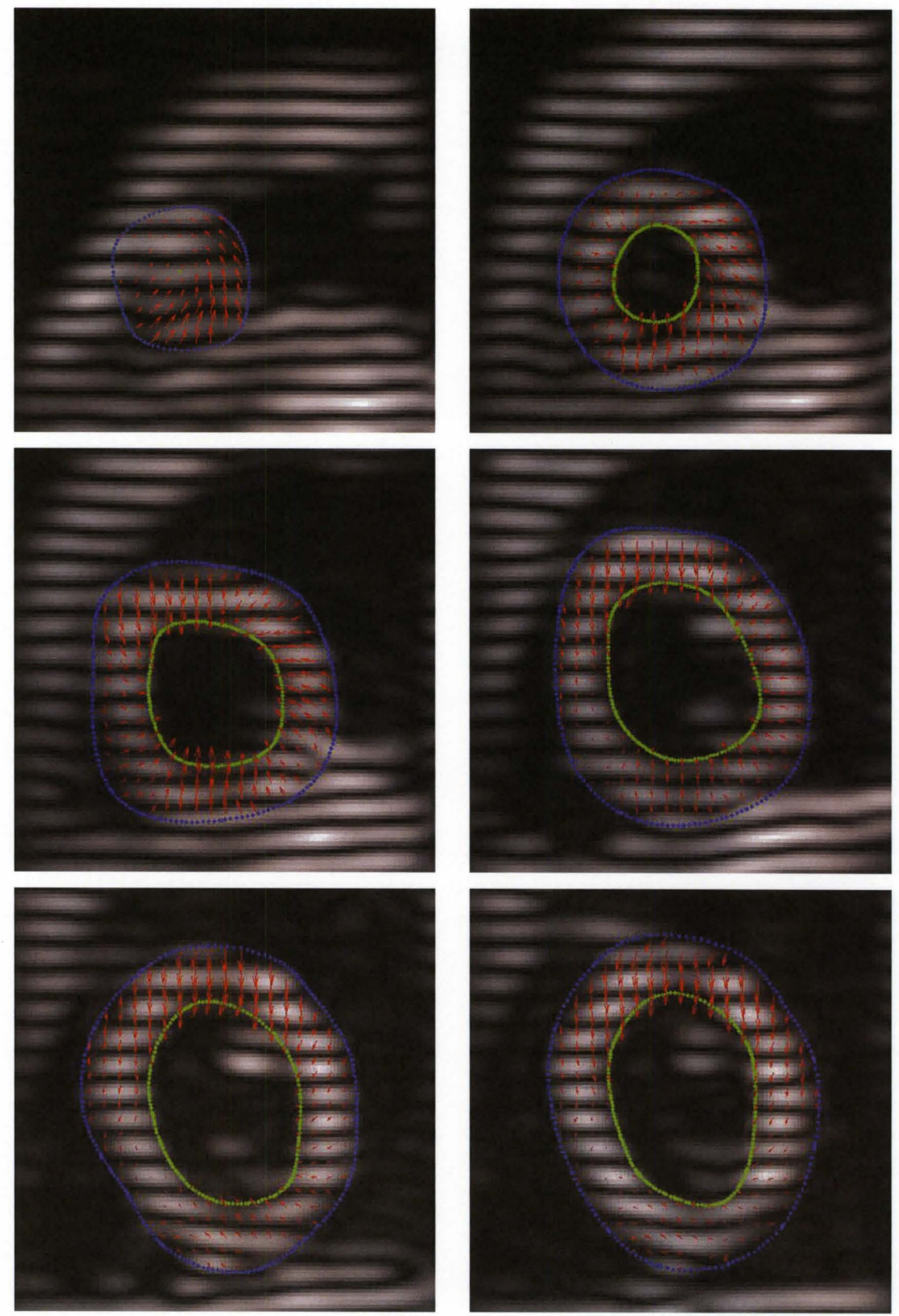

FIGURE 63-End systolic motion field on slices 1, 3, 5, 7, 9, 10 for data set 6 (row 1: apex, row 2: mid-ventricular, row 3: base). 

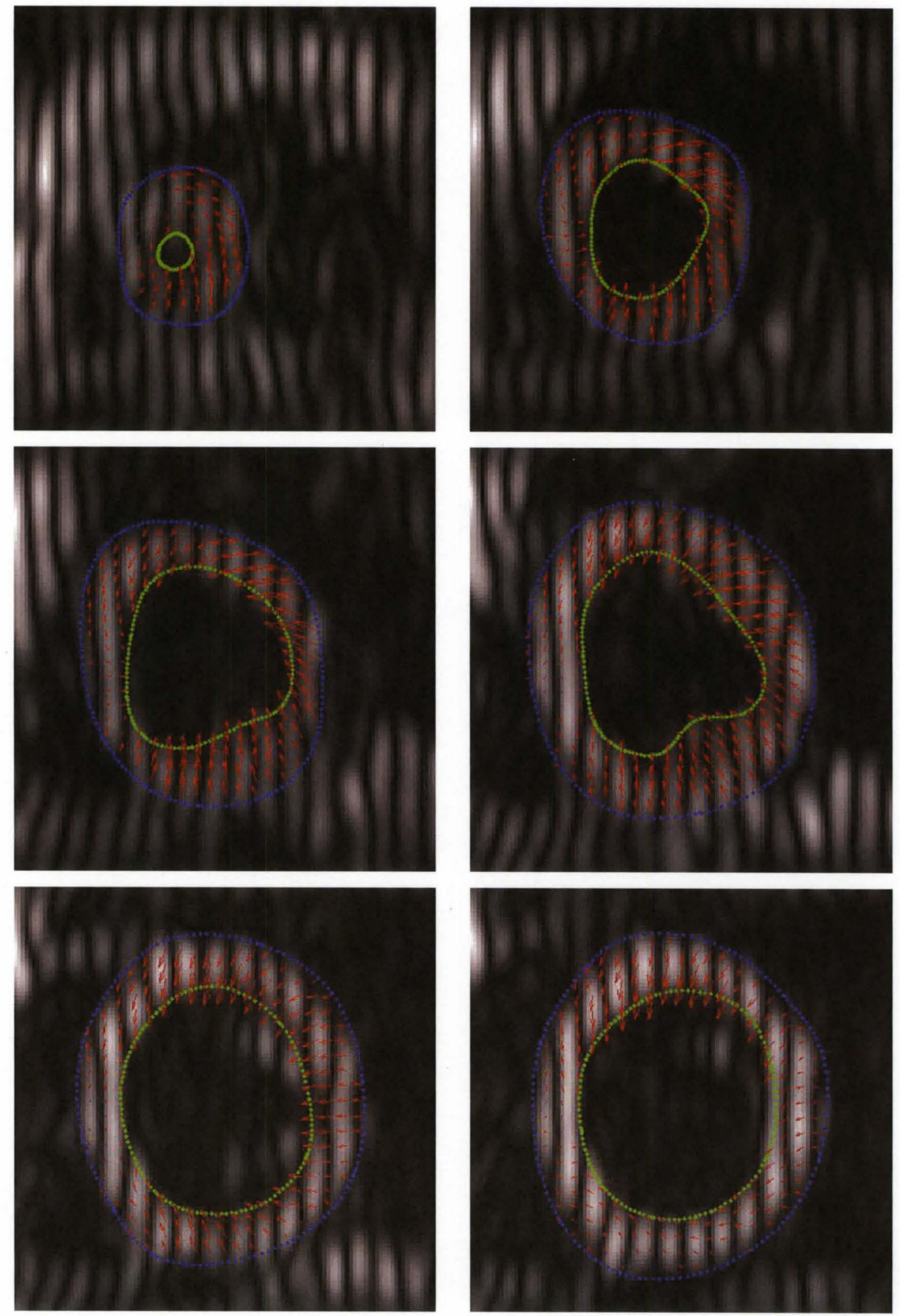

FIGURE 64-End systolic motion field on slices 1, 3, 5, 7, 9, 10 for data set 7 (row 1: apex, row 2: mid-ventricular, row 3: base). 


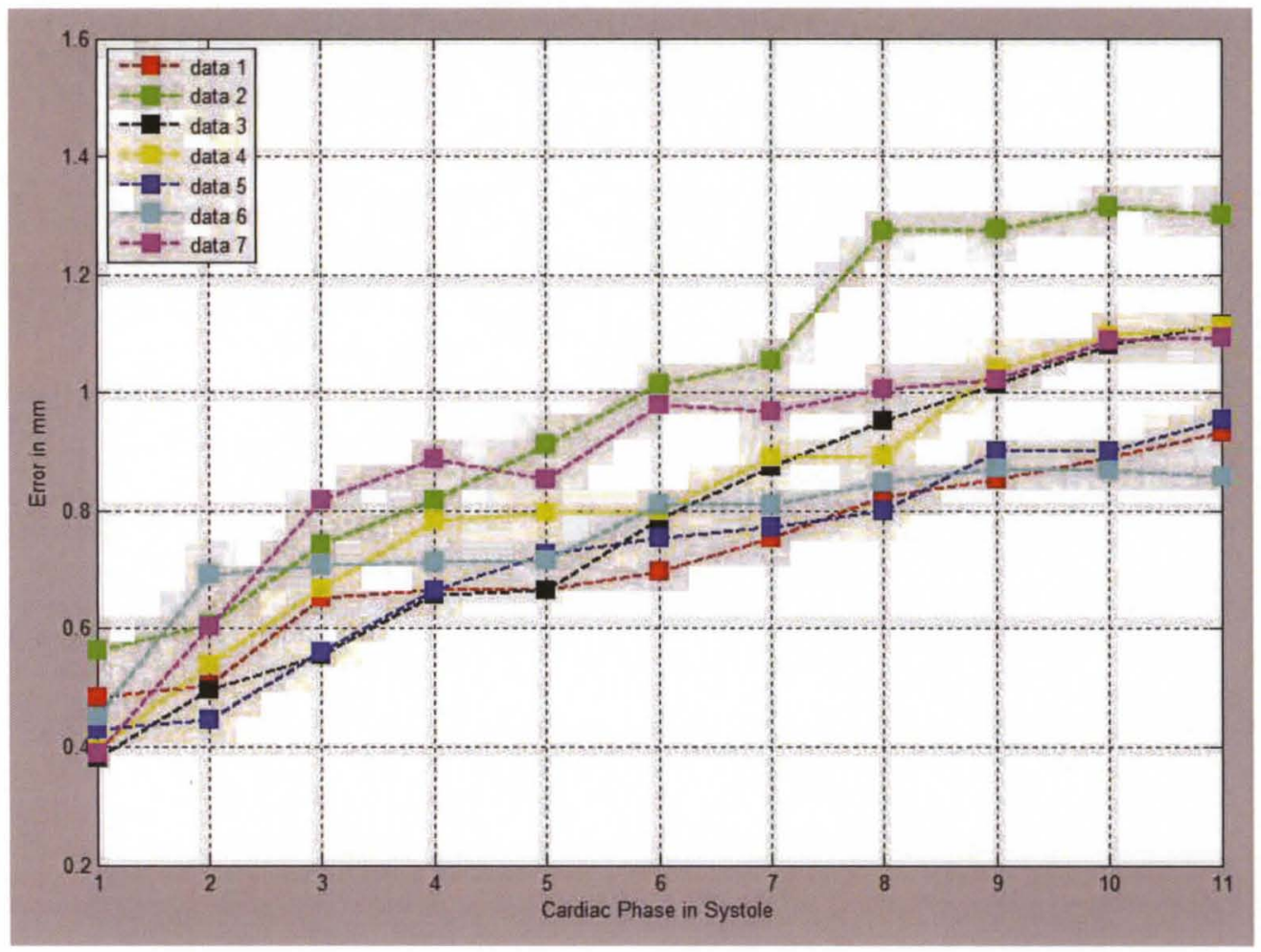

FIGURE 65-3D SinMod's average error as a function of time for determining tag line displacements during systole for 7 in-vivo data sets. Results for data sets $1,2,3,4$, 5,6 , and 7 are differentiated with red, green, black, yellow, blue, cyan, and magenta colors. Please note that the error for each time point was calculated from the error between warped tag line locations and manually delineated tag lines on all image slices. Also, note that the manually delineated tag lines at the reference frame were warped to all subsequent frames and results were compared with the actual location of tag lines. This explains the reason for the increased error in later phases. 


\section{TABLE 9}

The average circumferential shortening error between results from 3D SinMod and $3 \mathrm{D}$ HARP for slices 2 to 10 over all cardiac phases. The error is in percentage.

\begin{tabular}{|l|l|l|l|l|l|l|l|}
\hline & data 1 & data 2 & data 3 & data 4 & data 5 & data 6 & data 7 \\
\hline slice 2 & 2.12 & 3.36 & 1.55 & 1.09 & 0.93 & 0.91 & 0.94 \\
\hline slice 3 & 1.43 & 2.84 & 0.90 & 1.53 & 0.71 & 0.69 & 0.81 \\
\hline slice 4 & 1.90 & 1.71 & 0.74 & 0.79 & 0.45 & 0.71 & 0.80 \\
\hline slice 5 & 0.51 & 0.93 & 0.77 & 0.41 & 0.47 & 0.80 & 0.82 \\
\hline slice 6 & 0.31 & 0.87 & 0.50 & 0.35 & 0.42 & 0.90 & 0.54 \\
\hline slice 7 & 0.24 & 0.88 & 0.38 & 0.51 & 0.52 & 0.97 & 0.49 \\
\hline slice 8 & 0.95 & 1.00 & 0.94 & 0.40 & 0.59 & 0.81 & 0.74 \\
\hline slice 9 & 0.76 & 0.85 & 1.02 & 0.85 & 0.97 & 0.66 & 0.85 \\
\hline slice 10 & 0.67 & 1.00 & 1.94 & 1.57 & 1.95 & 0.70 & 1.27 \\
\hline
\end{tabular}

\section{Circumferential Shortening}

Circumferential shortening is the relative change in length of a mid-wall contour with respect to the reference length at end-diastole.

$$
\text { Circumferential Shortening }=\frac{L_{c u r}-L_{r e f}}{L_{r e f}}
$$

where $L_{c u r}$ is the mid-wall contour length at the current frame, and $L_{r e f}$ is the midwall contour length at the reference frame. Figures 66 to 79 show the circumferential shortening percentage at different cardiac phases for different slices (from slice 2 to slice 10) for each data set. The red plots are the results from 3D SinMod and the green plots are the results from 3D HARP. There is a good degree of correspondence present. Table 9 shows the average circumferential shortening error between the results from 3D SinMod and 3D HARP. 

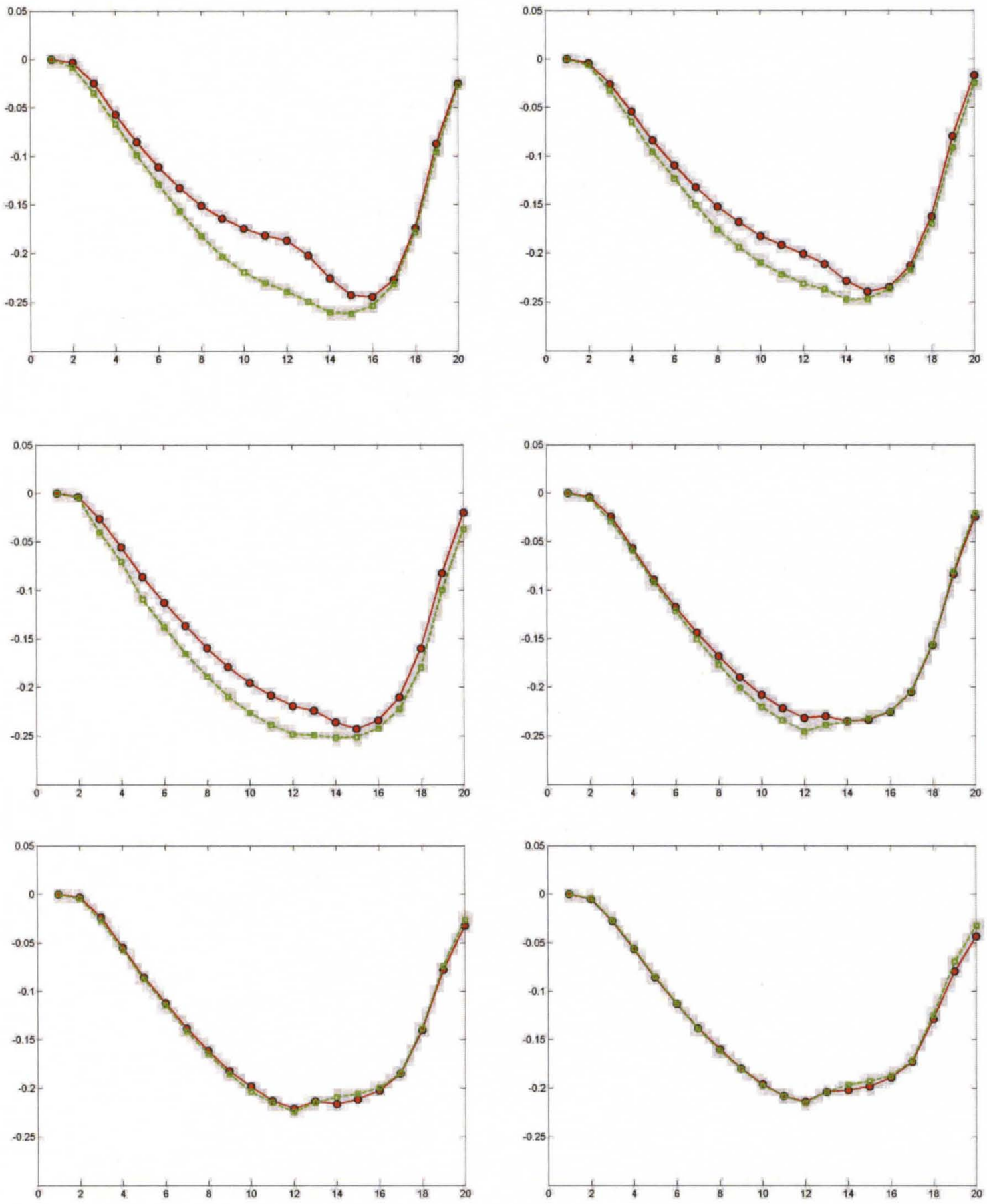

FIGURE 66 - Circumferential Shortening (Equation 69) at different phases during the cardiac cycle for data set 1 . Traversing from top-left to the bottom-right are results for slices 2 to 7 . 

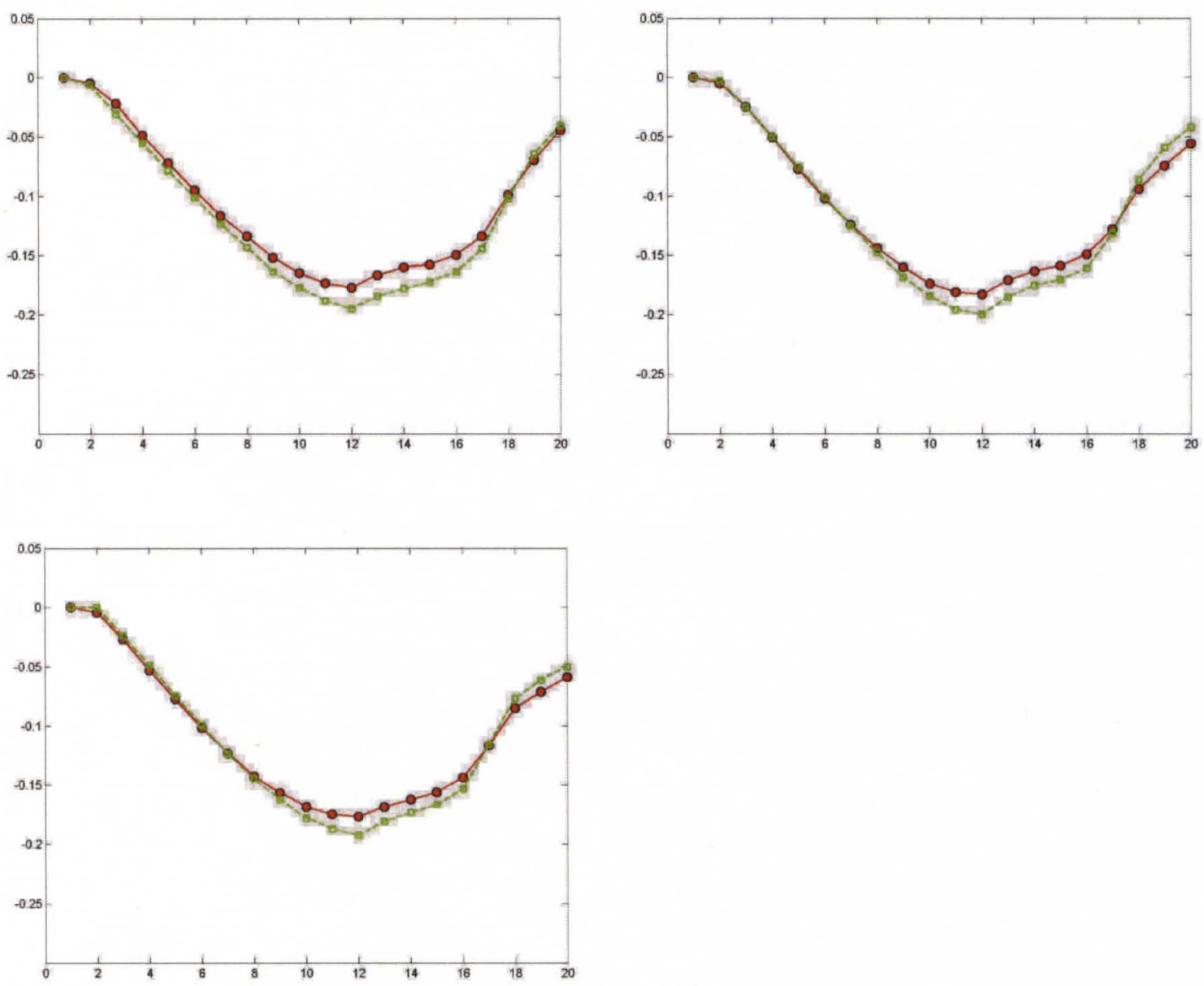

FIGURE 67-Circumferential Shortening (Equation 69) at different phases during the cardiac cycle for data set 1 . Traversing from top-left to the bottom-right are results for slices 8 to 10 . 

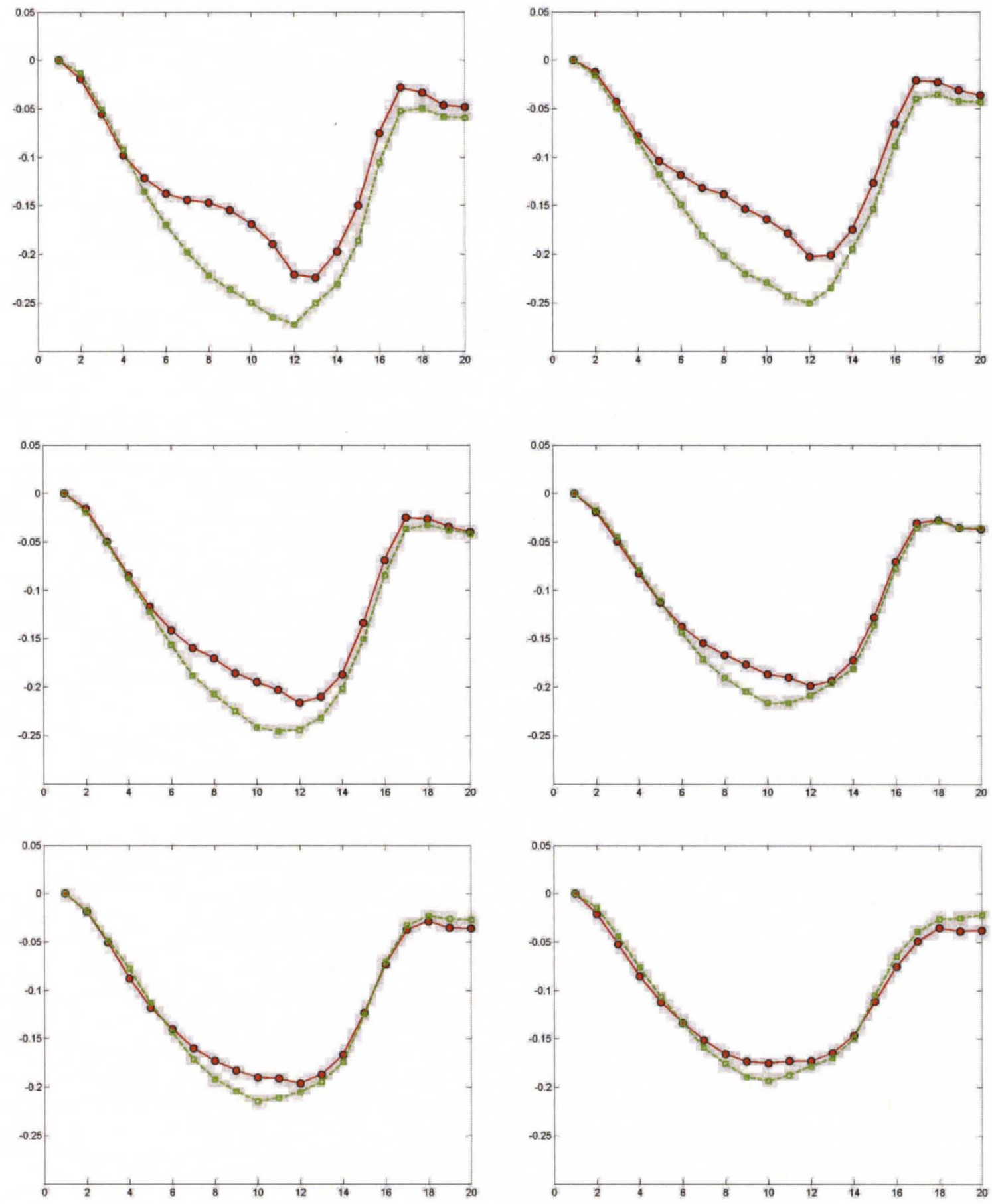

FIGURE 68 - Circumferential Shortening (Equation 69) at different phases during the cardiac cycle for data set 2 . Traversing from top-left to the bottom-right are results for slices 2 to 7 . 

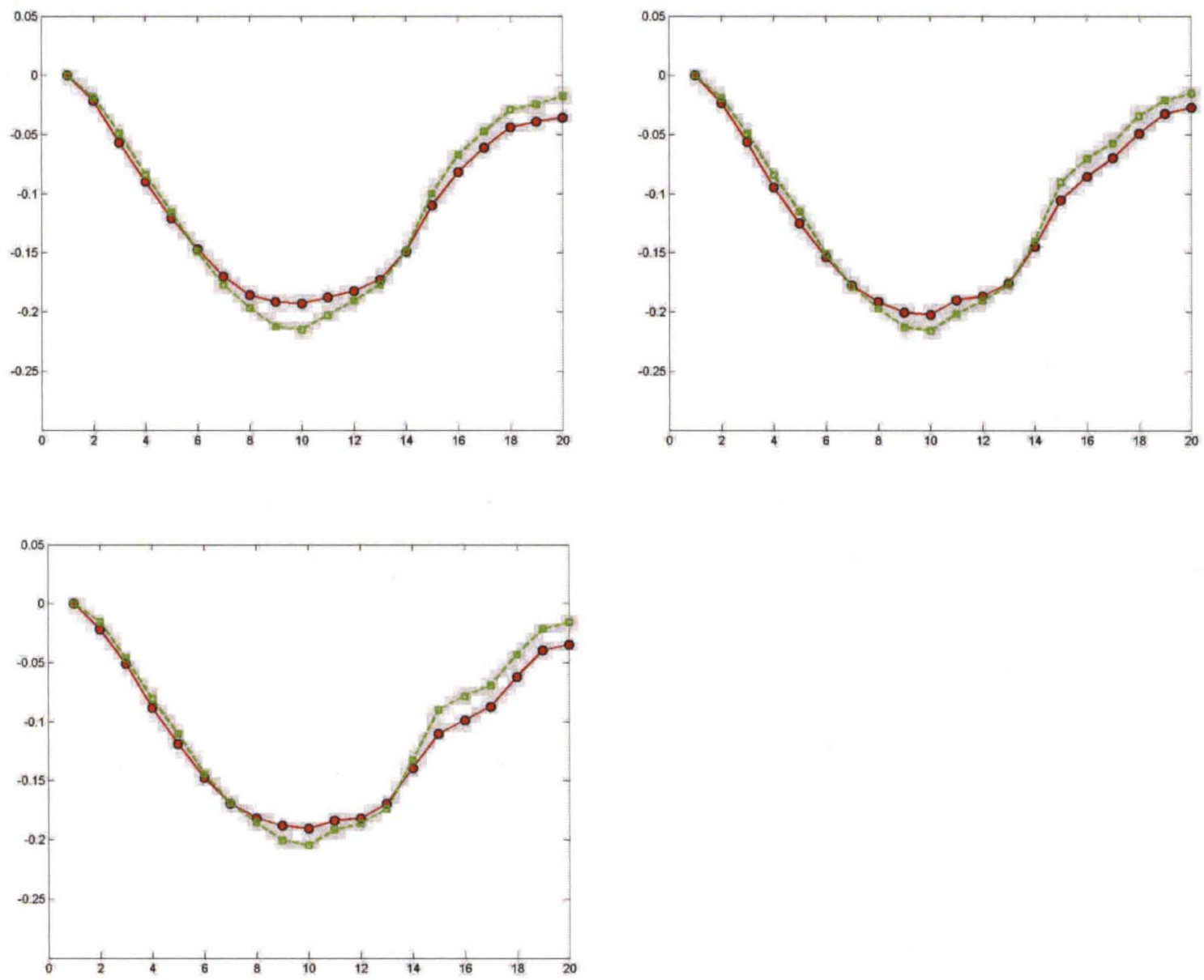

FIGURE 69 - Circumferential Shortening (Equation 69) at different phases during the cardiac cycle for data set 2 . Traversing from top-left to the bottom-right are results for slices 8 to 10 . 

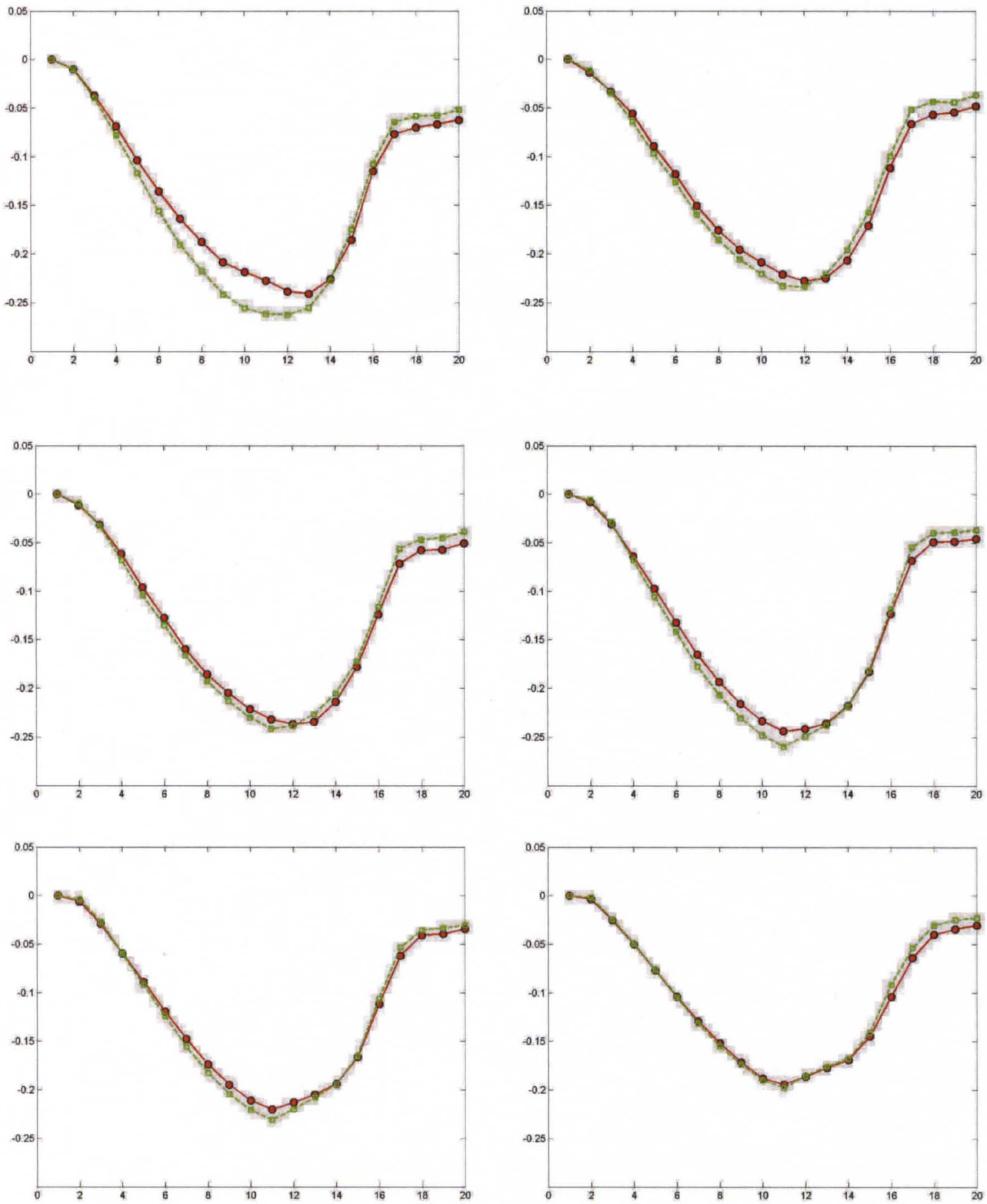

FIGURE 70 - Circumferential Shortening (Equation 69) at different phases during the cardiac cycle for data set 3 . Traversing from top-left to the bottom-right are results for slices 2 to 7 . 

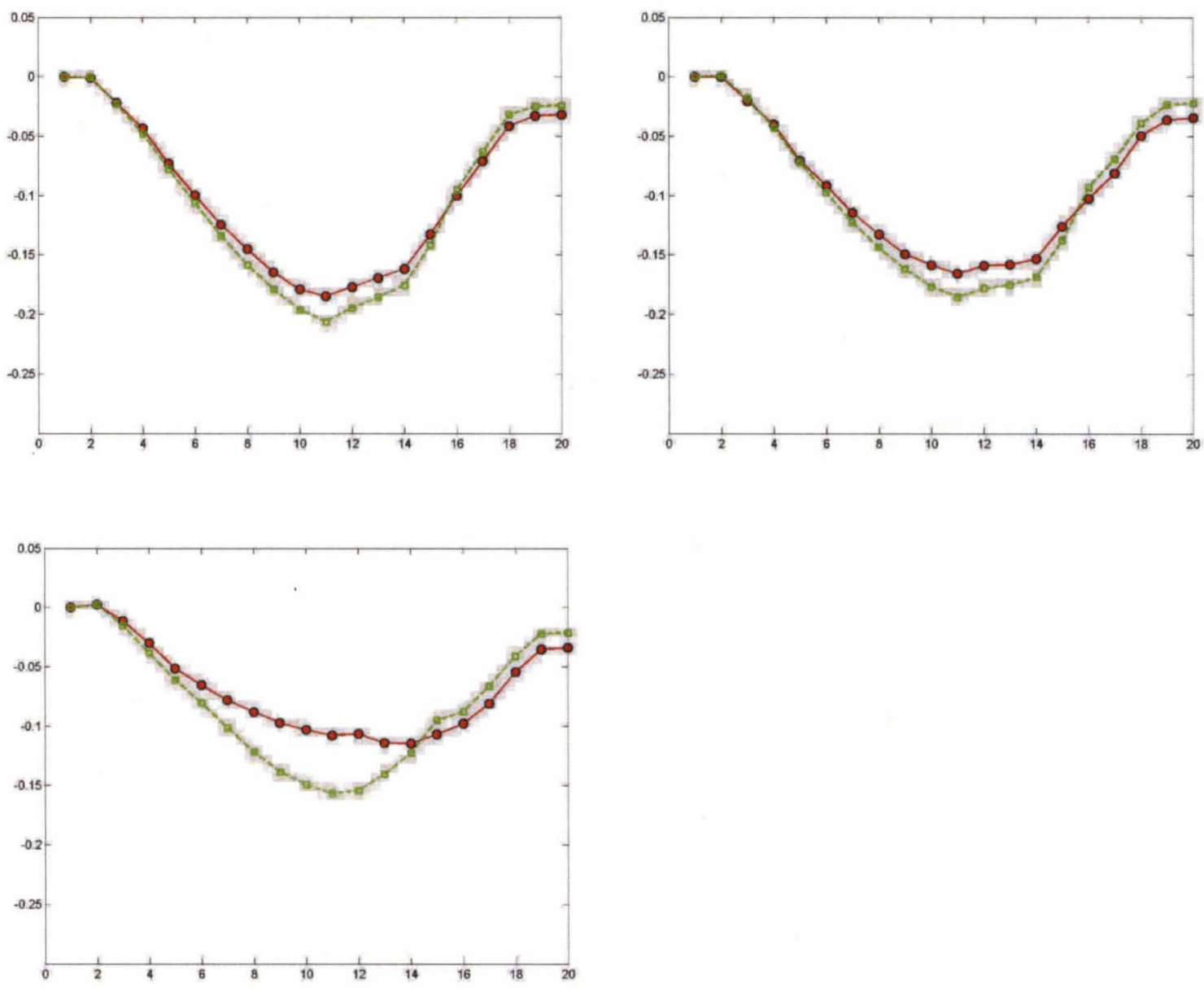

FIGURE 71 - Circumferential Shortening (Equation 69) at different phases during the cardiac cycle for data set 3 . Traversing from top-left to the bottom-right are results for slices 8 to 10 . 

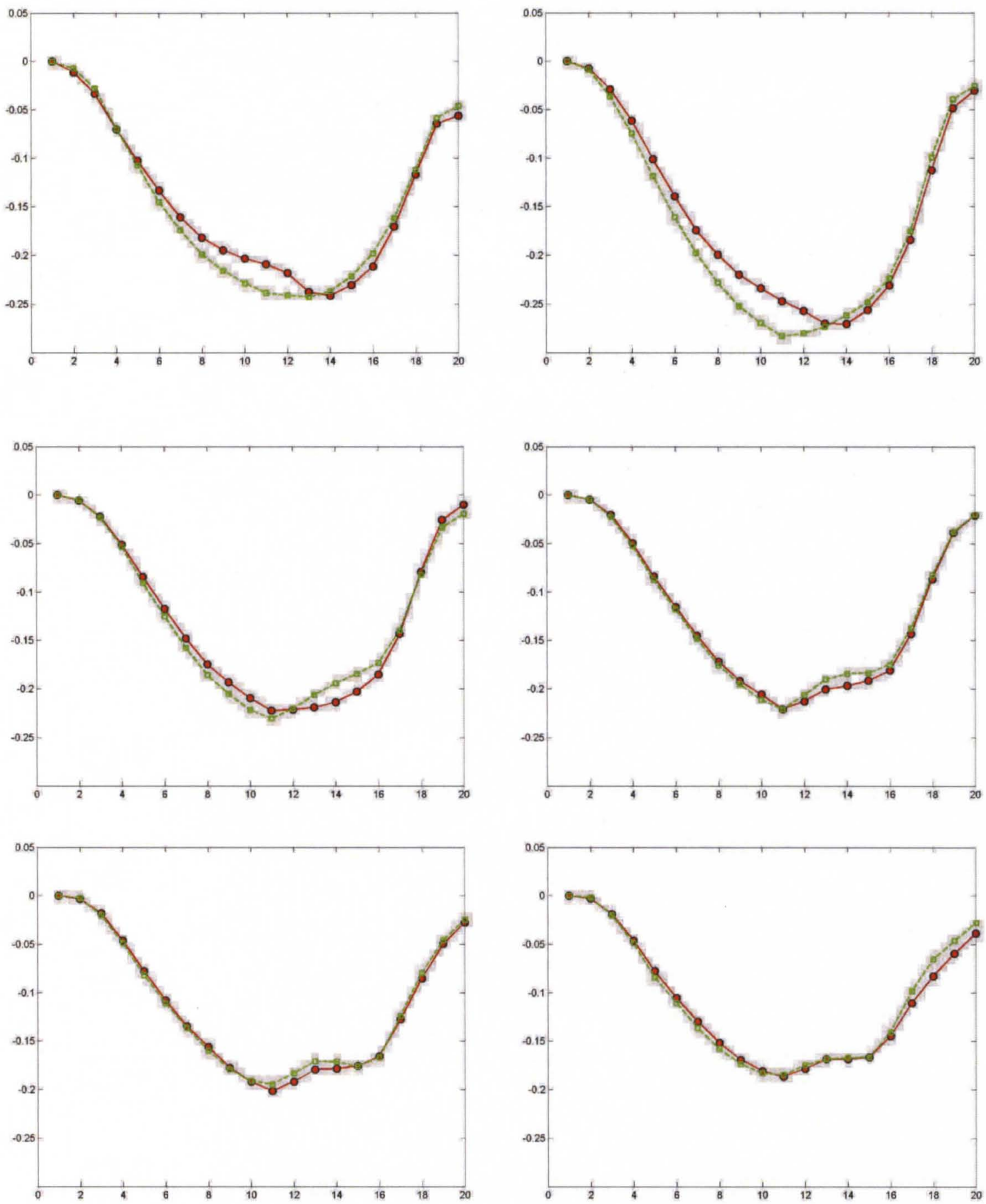

FIGURE 72 - Circumferential Shortening (Equation 69) at different phases during the cardiac cycle for data set 4 . Traversing from top-left to the bottom-right are results for slices 2 to 7 . 

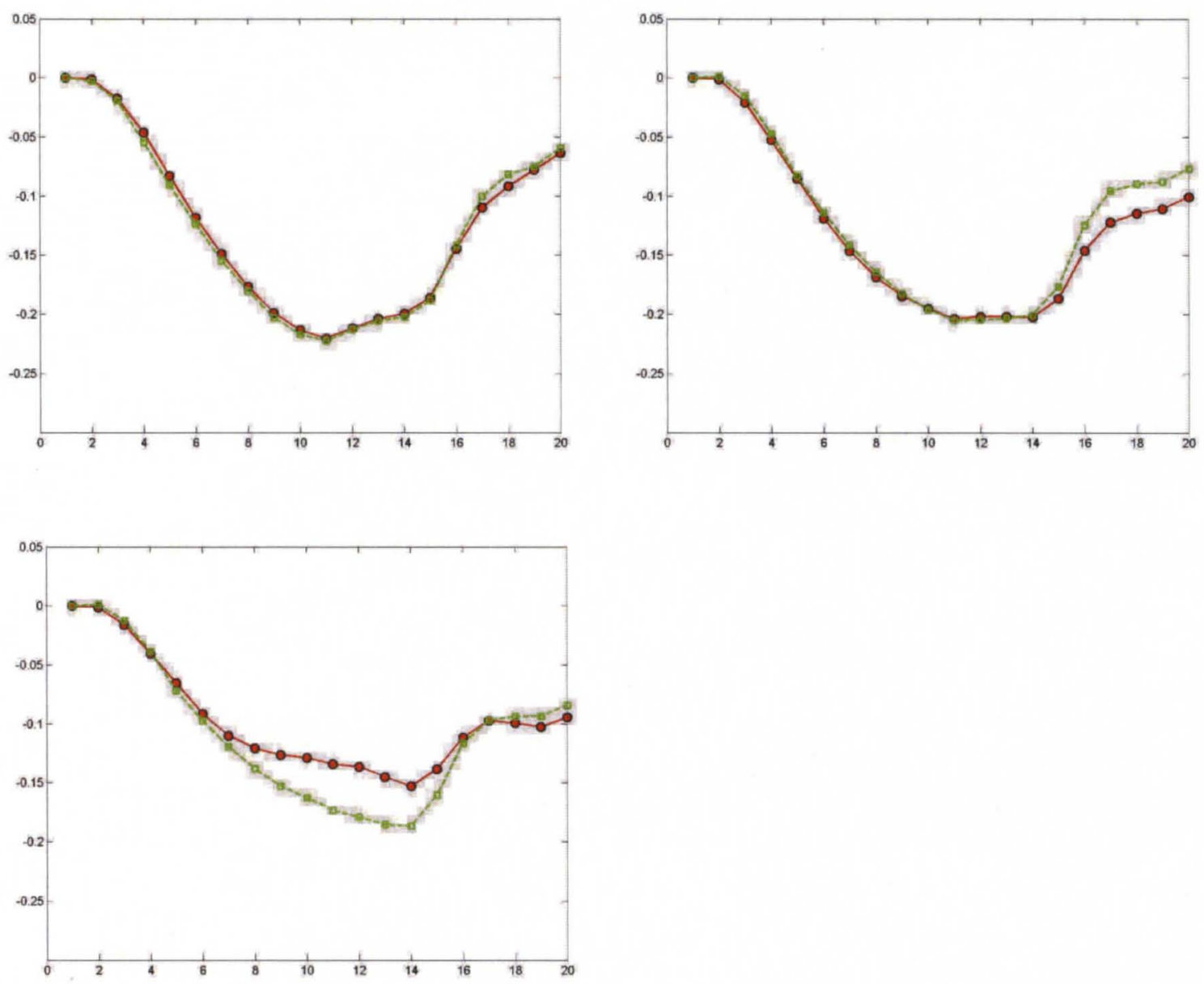

FIGURE 73 - Circumferential Shortening (Equation 69) at different phases during the cardiac cycle for data set 4 . Traversing from top-left to the bottom-right are results for slices 8 to 10 . 

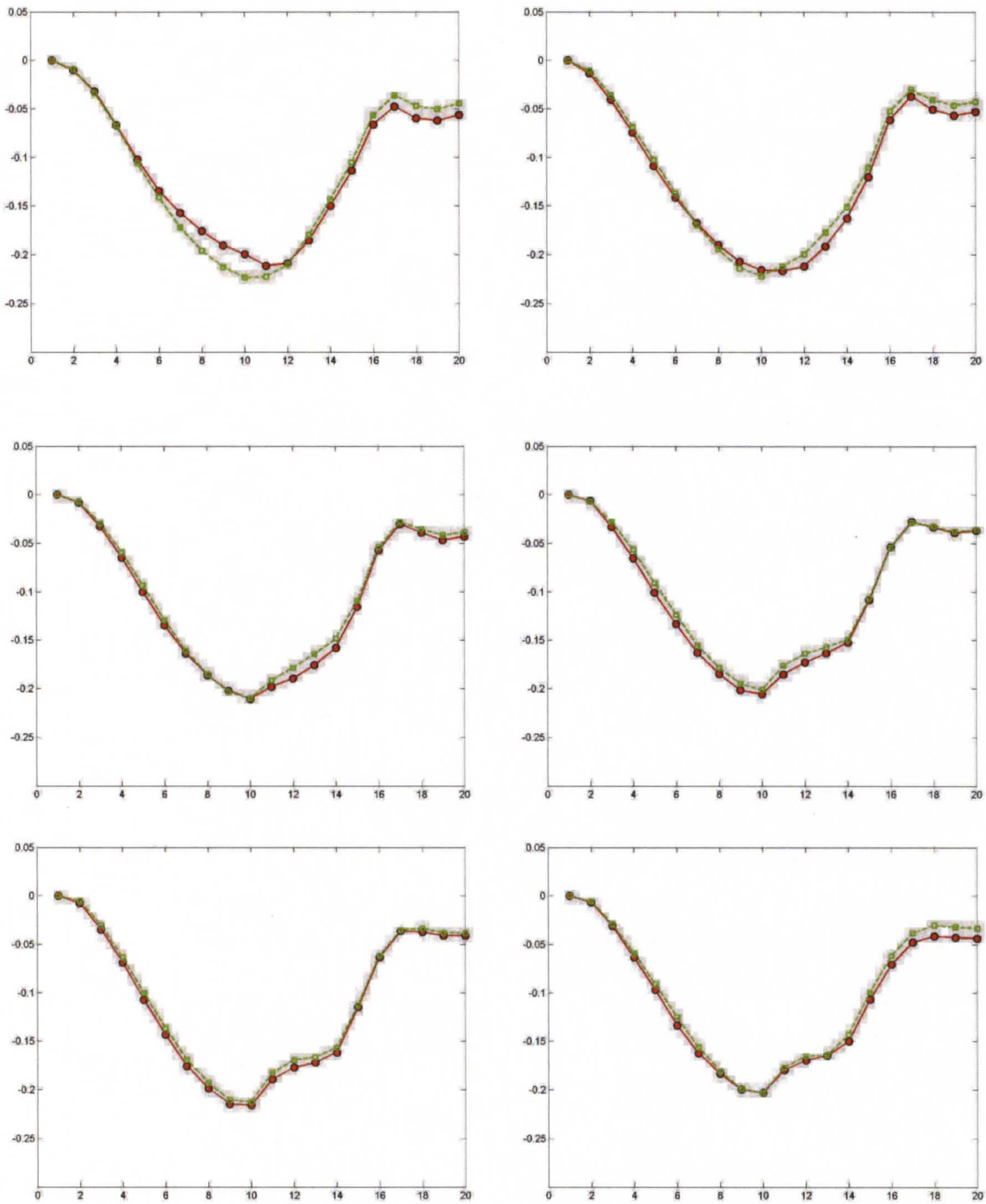

FIGURE 74-Circumferential Shortening (Equation 69) at different phases during the cardiac cycle for data set 5 . Traversing from top-left to the bottom-right are results for slices 2 to 7 . 

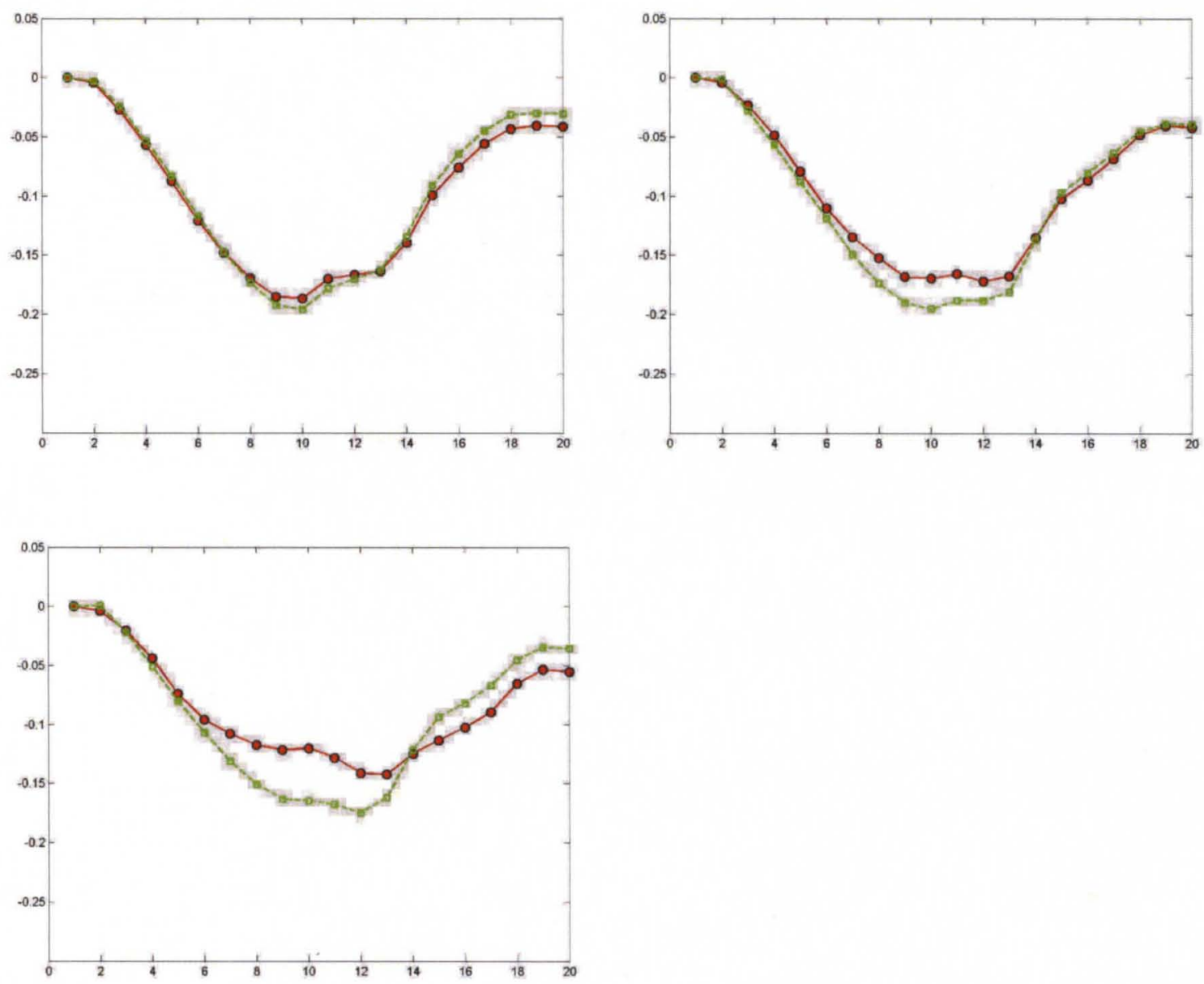

FIGURE 75 - Circumferential Shortening (Equation 69) at different phases during the cardiac cycle for data set 5 . Traversing from top-left to the bottom-right are results for slices 8 to 10 . 

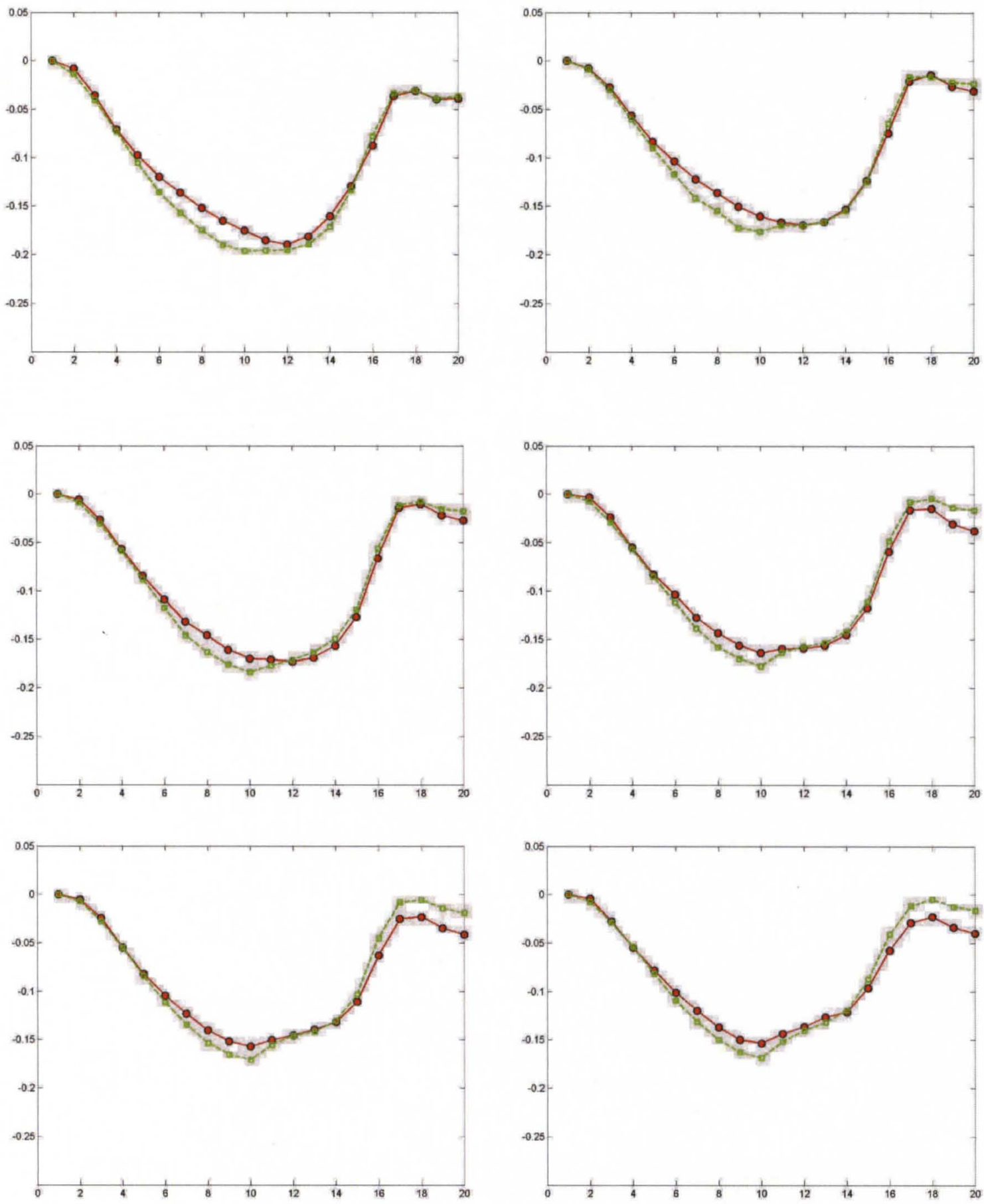

FIGURE 76 - Circumferential Shortening (Equation 69) at different phases during the cardiac cycle for data set 6 . Traversing from top-left to the bottom-right are results for slices 2 to 7 . 

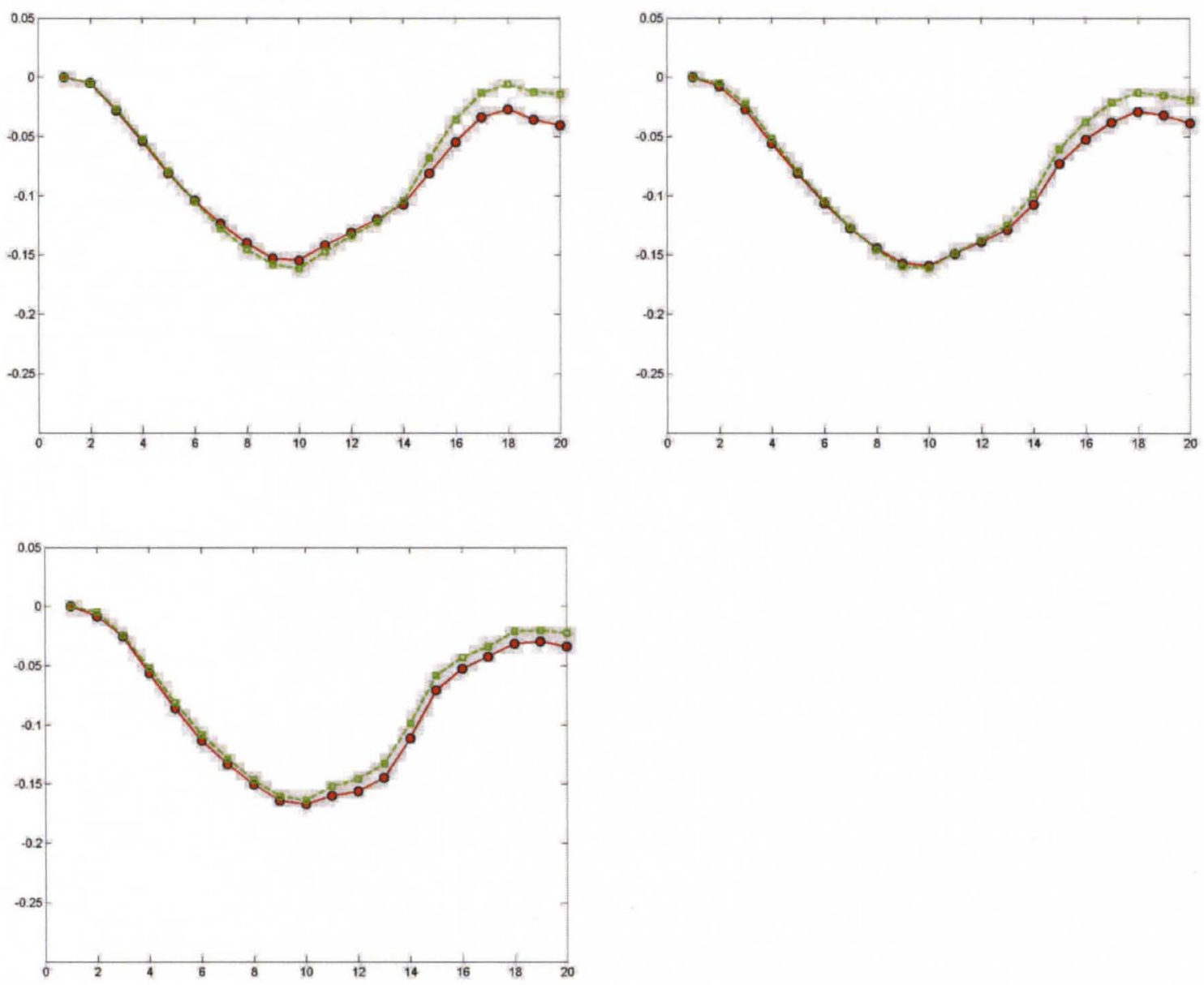

FIGURE 77 -Circumferential Shortening (Equation 69) at different phases during the cardiac cycle for data set 6 . Traversing from top-left to the bottom-right are results for slices 8 to 10 . 

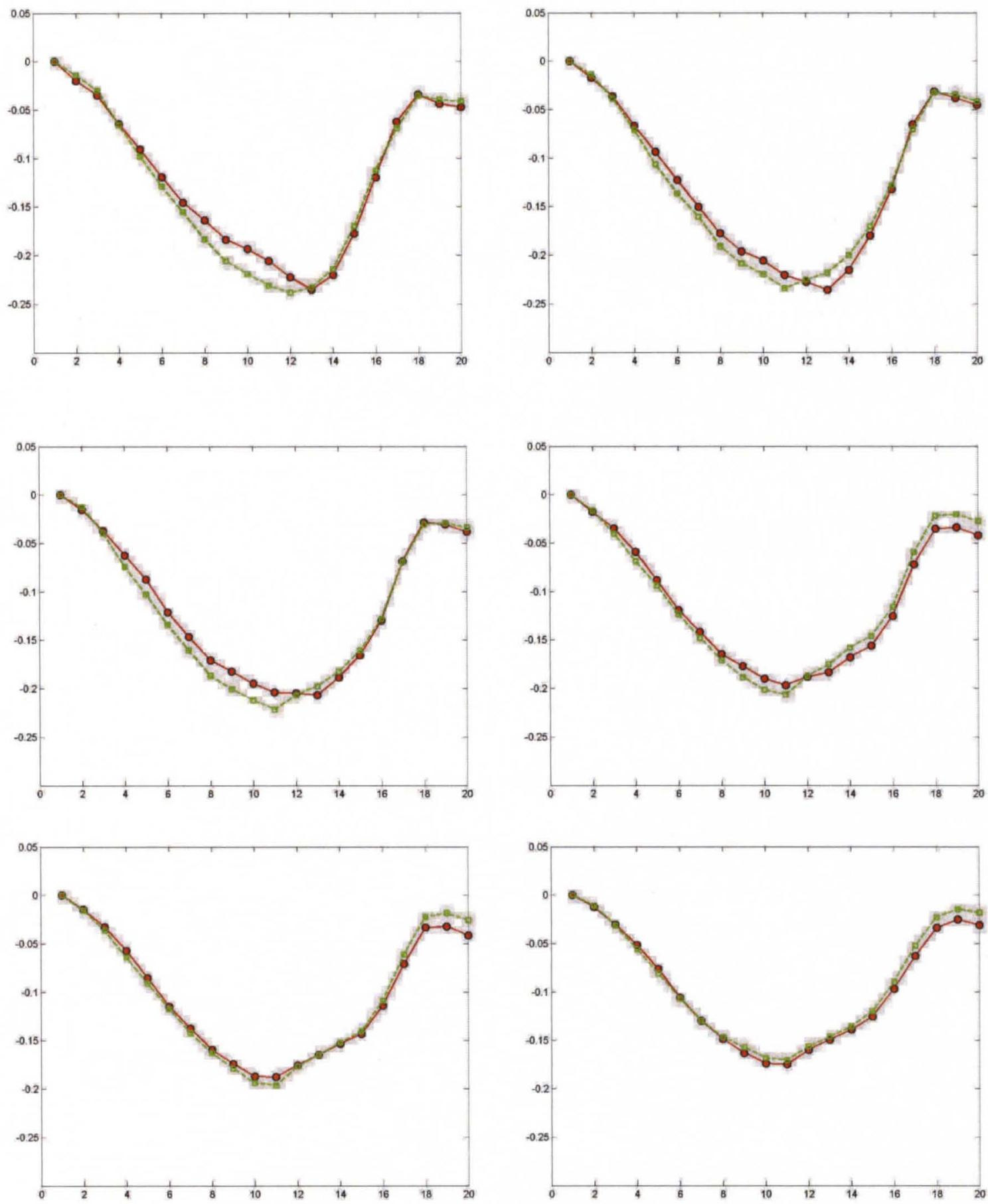

FIGURE 78 - Circumferential Shortening (Equation 69) at different phases during the cardiac cycle for data set 7 . Traversing from top-left to the bottom-right are results for slices 2 to 7 . 

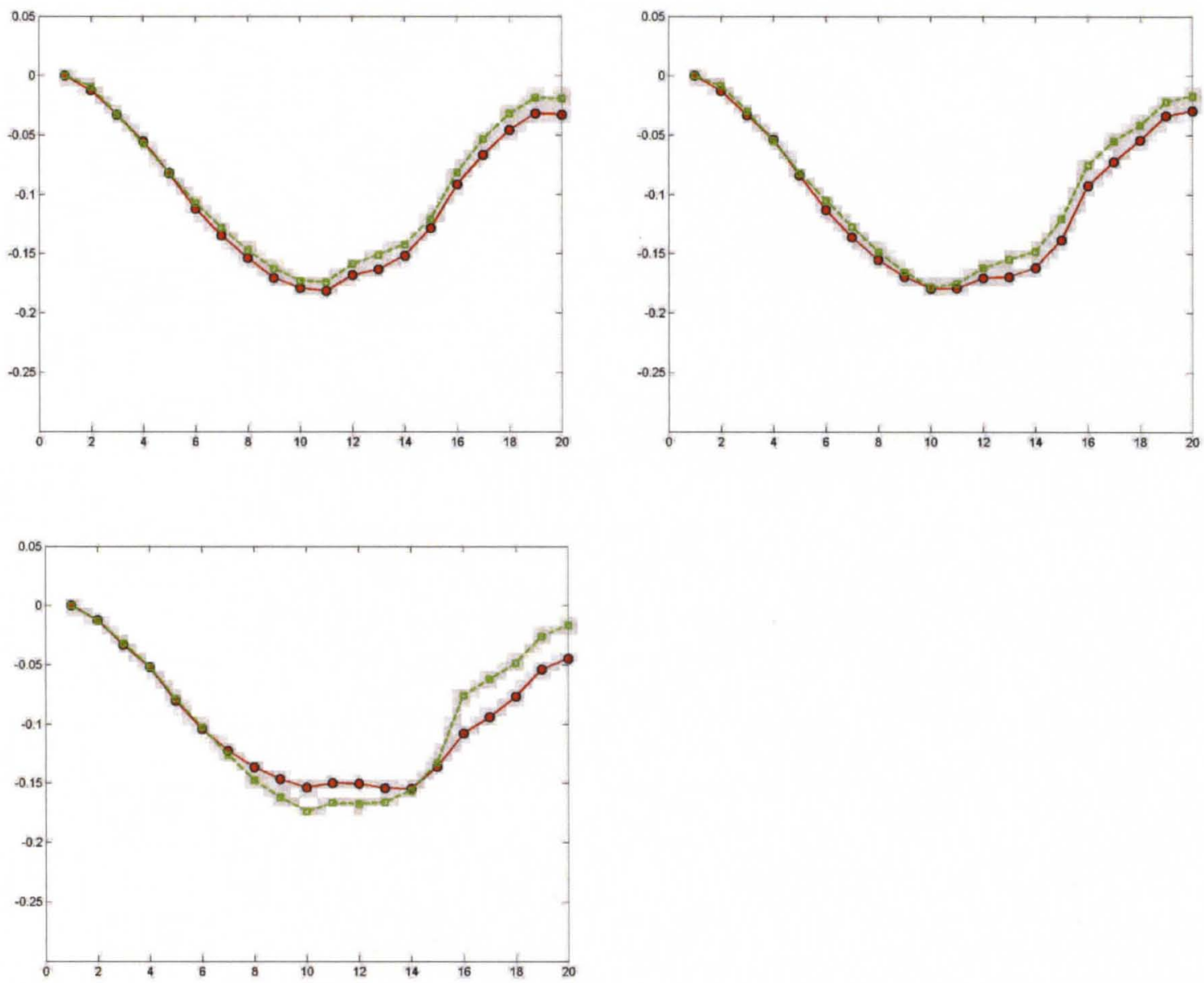

FIGURE 79 - Circumferential Shortening (Equation 69) at different phases during the cardiac cycle for data set 7 . Traversing from top-left to the bottom-right are results for slices 8 to 10 . 


\section{Strains}

In order to analyze regional strain patterns in the myocardium, the LV and RV are typically divided into segments and each of the directional strains (e.g., radial, circumferential, and/or longitudinal) is averaged over the segment. In $3 \mathrm{D}$, the 16 segment and 17 segment models as recommended by the American Society of Echocardiography should be used [150]. In the 16 segment model, the LV is divided into 6 basal, 6 mid-ventricular, and 4 apical regions. In the 17 segment model, the apex comprises the $17^{\text {th }}$ segment $[85,97,99,105,124,136,137]$. In 3D, the RV can be divided into 3 layers in the long-axis direction and each layer is further divided into 3 segments $[97,151]$. However other finer or sparser partitions have also been utilized. For 2D analysis, typically, mid-ventricular strains for 4-8 regions are reported $[70,78,122,135]$.

In [17], the division along the long-axis consisted of four layers: basal, midcavity, apical, and apex as shown in Figure 80. Based on the location of the LV/RV junctions, the basal and mid-cavity portions of the left ventricle were each further divided into six regions in the short-axis view: anterior (A), antero-septal (AS), inferoseptal (IS), inferior (I), posterior (P), and lateral (L) as shown in Figure 81. The 17 segments refer to: basal anterior (segment 1), basal antero-septal (segment 2), basal infero-septal (segment 3), basal inferior (segment 4), basal posterior (segment 5), basal lateral (segment 6), mid-ventricular anterior (segment 7), mid-ventricular antero-septal (segment 8), mid-ventricular infero-septal (segment 9), mid-ventricular inferior (segment 10), mid-ventricular posterior (segment 11), mid-ventricular lateral (segment 12), apical anterior (segment 13), apical septal (segment 14), apical inferior (segment 15), apical lateral (segment 16), and apex (segment 17).

Lagrangian strain values for LV data sets from 7 healthy subjects have been calculated. The histogram of Jacobian for all data sets are shown in Figures 82 and 83. Jacobian represents the volume change for a small cube of unit volume at the 


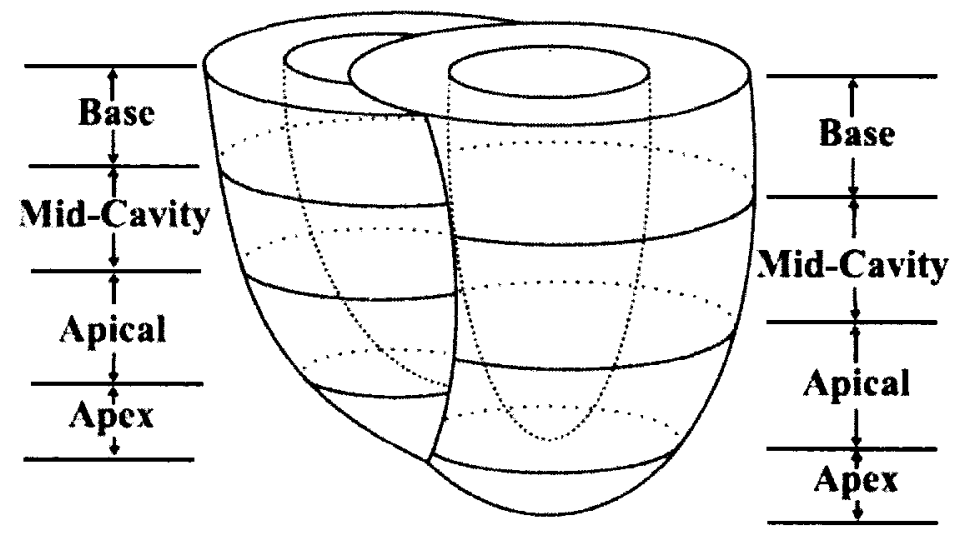

FIGURE 80 -Geometrical representation of the regional divisions for strain analysis for both the left and right ventricles in the LA view [17].

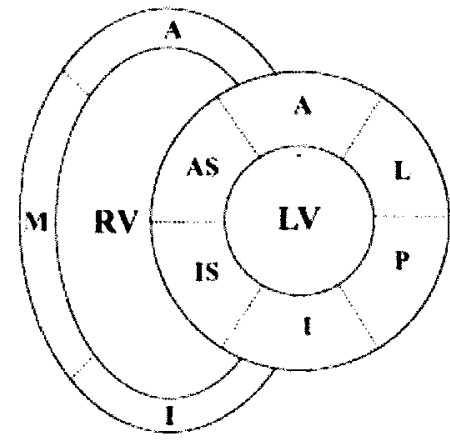

(a)

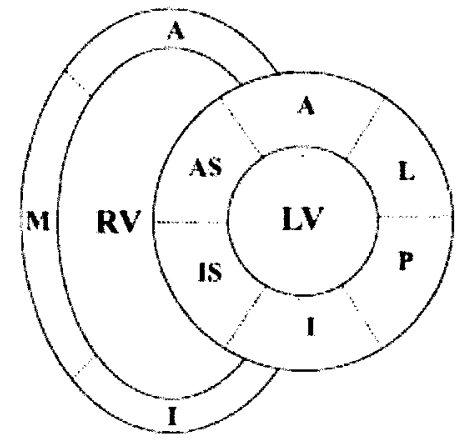

(b)

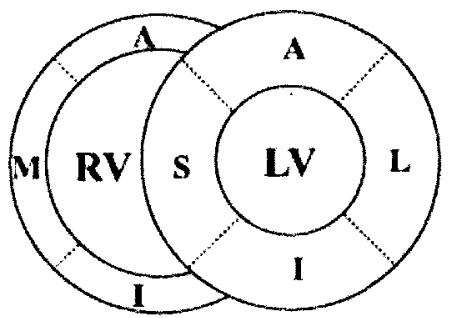

(c)

FIGURE 81 - Geometrical representation of the regional divisions for strain analysis for both the left and right ventricles in the SA view. Illustrated are (a) the basal region, (b) the mid-cavity region, and (c) the apical region [17]. 

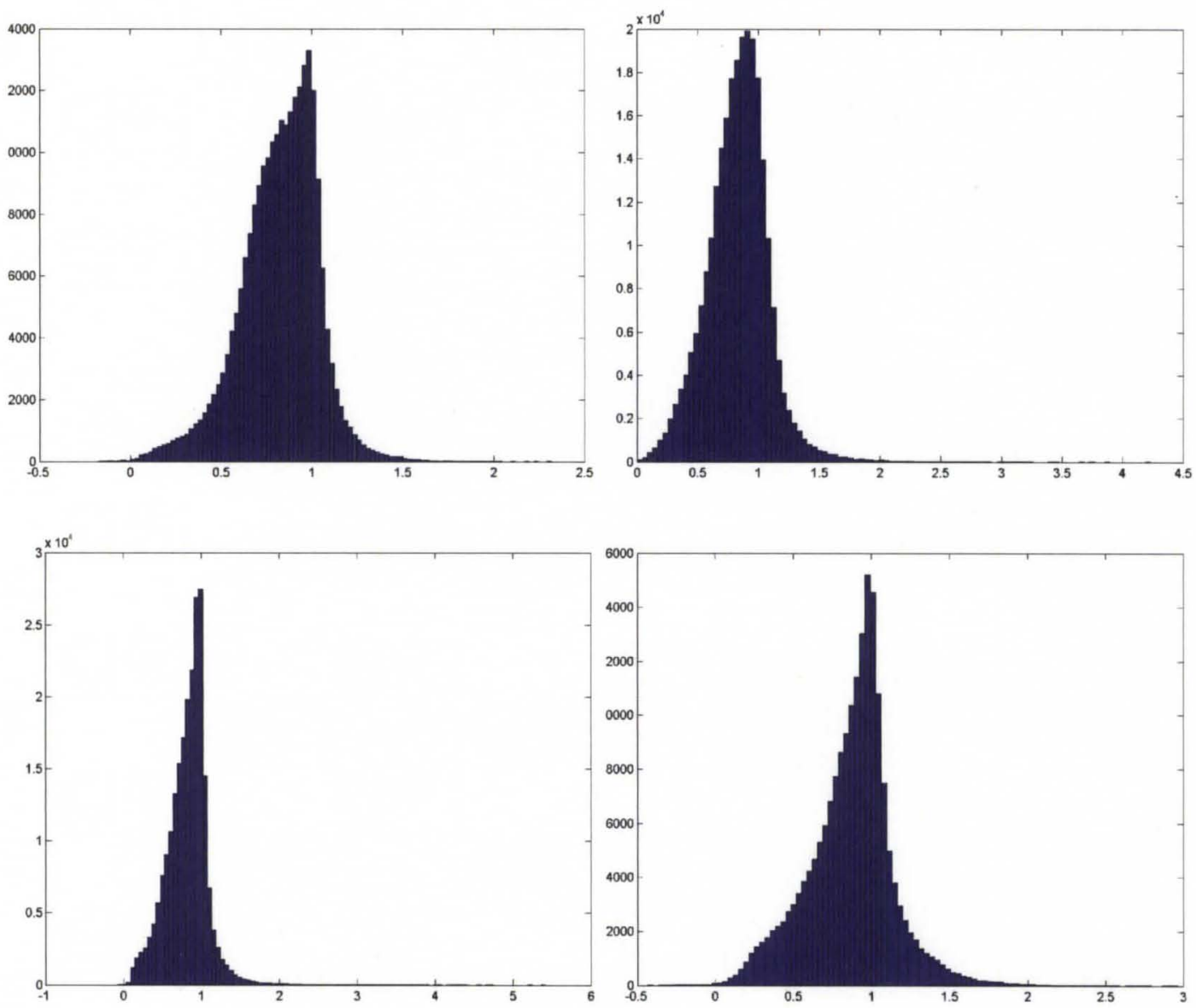

FIGURE 82 - Histogram of the Jacobian of deformation (see Equation 13) as calculated from 3D SinMod for different data sets. Note that the Jacobian of the deformation gradient tensor for all myocardial points in space and time for each of the $3 \mathrm{D}+\mathrm{t}$ data sets (10 slices and 10 temporal frames) are summarized in a single histogram. Traversing from top-left to the bottom-right are results for data sets 1 to 4 .

undeformed state. The average radial, circumferential, and longitudinal strain curves for different segments over systole averaged for all 7 data sets are shown in Figures 84 to 86. As may be observed, radial strains are positive in accordance to systolic thickening of the LV myocardium, whereas the circumferential and longitudinal strains are negative, in accordance to circumferential and longitudinal shortening during systole. In the radial direction very few measurements are available and therefore as expected significant noise is present. 

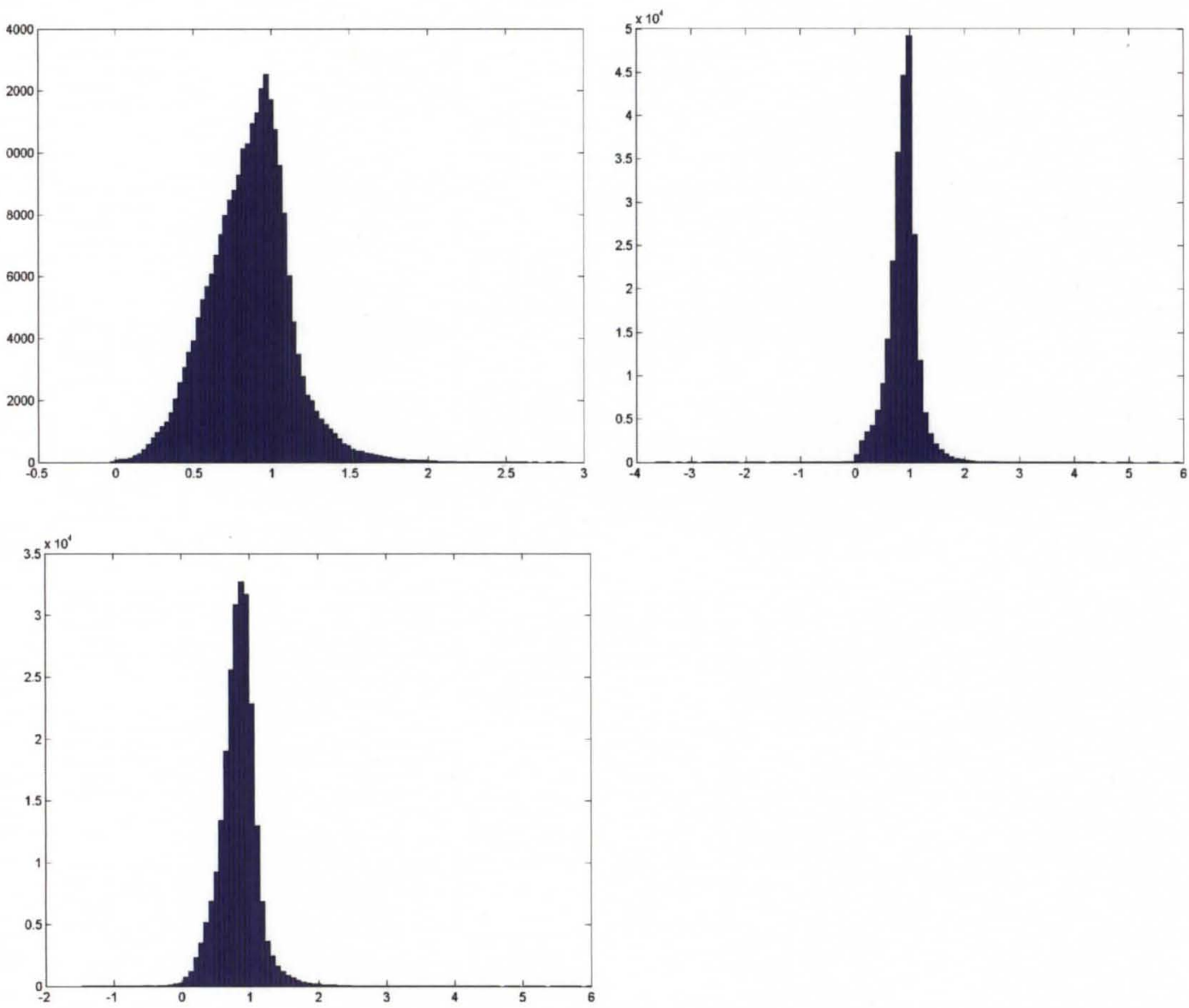

FIGURE 83 - Histogram of the Jacobian of deformation (see Equation 13) as calculated from 3D SinMod for different data sets. Note that the Jacobian of the deformation gradient tensor for all myocardial points in space and time for each of the $3 \mathrm{D}+\mathrm{t}$ data sets (10 slices and 10 temporal frames) are summarized in a single histogram. Traversing from top-left to the bottom-right are results for data sets 5 to 7 . 

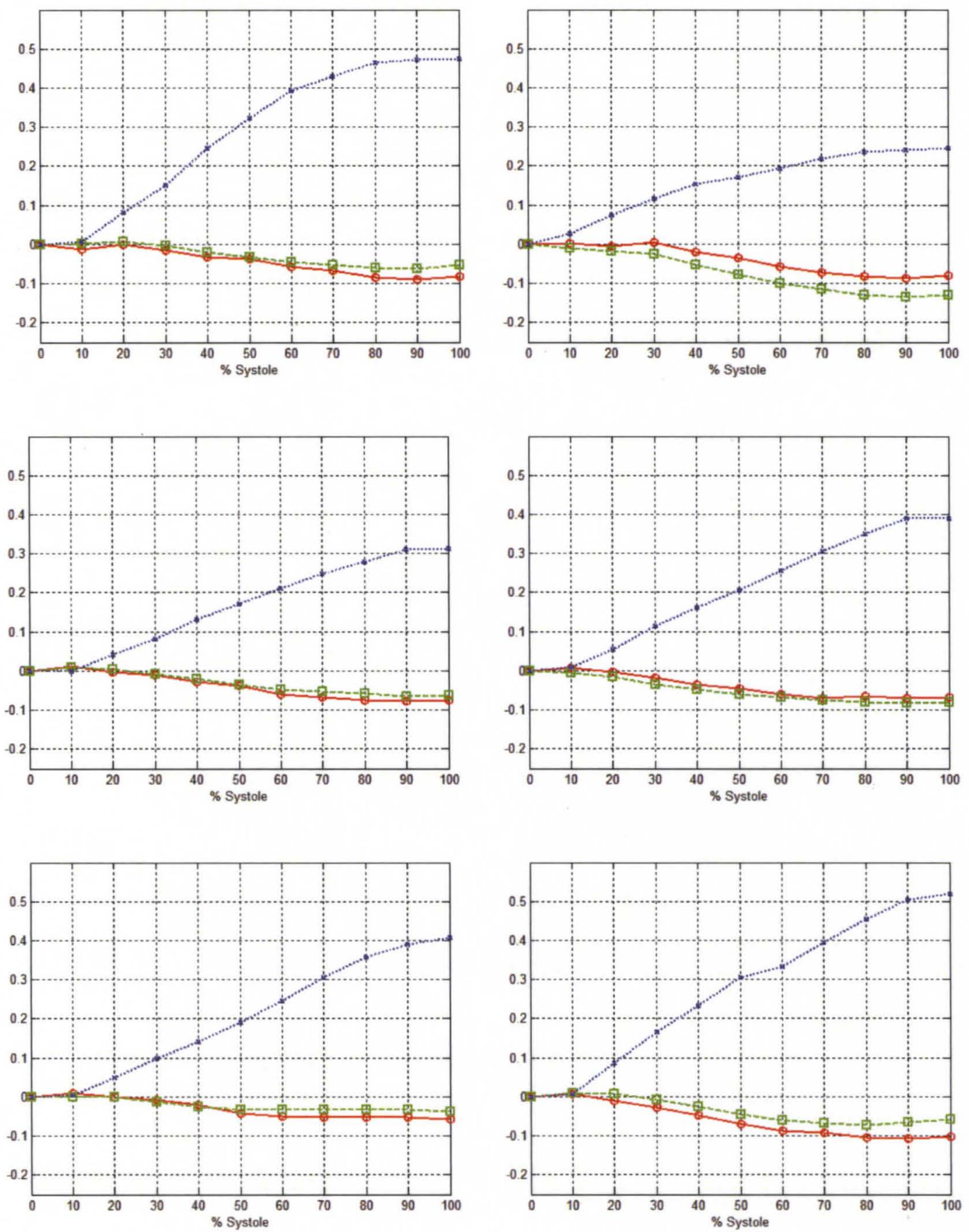

FIGURE 84 - The average radial, circumferential, and longitudinal strain curves during systole for all 7 data sets. Radial strain is shown in dotted blue line. Circumferential strain is shown in dashed green line. Longitudinal strain is shown in solid red line. Traversing from top-left to the bottom-right are results for basal anterior (segment 1), basal antero-septal (segment 2), basal infero-septal (segment 3), basal inferior (segment 4), basal posterior (segment 5), basal lateral (segment 6). 

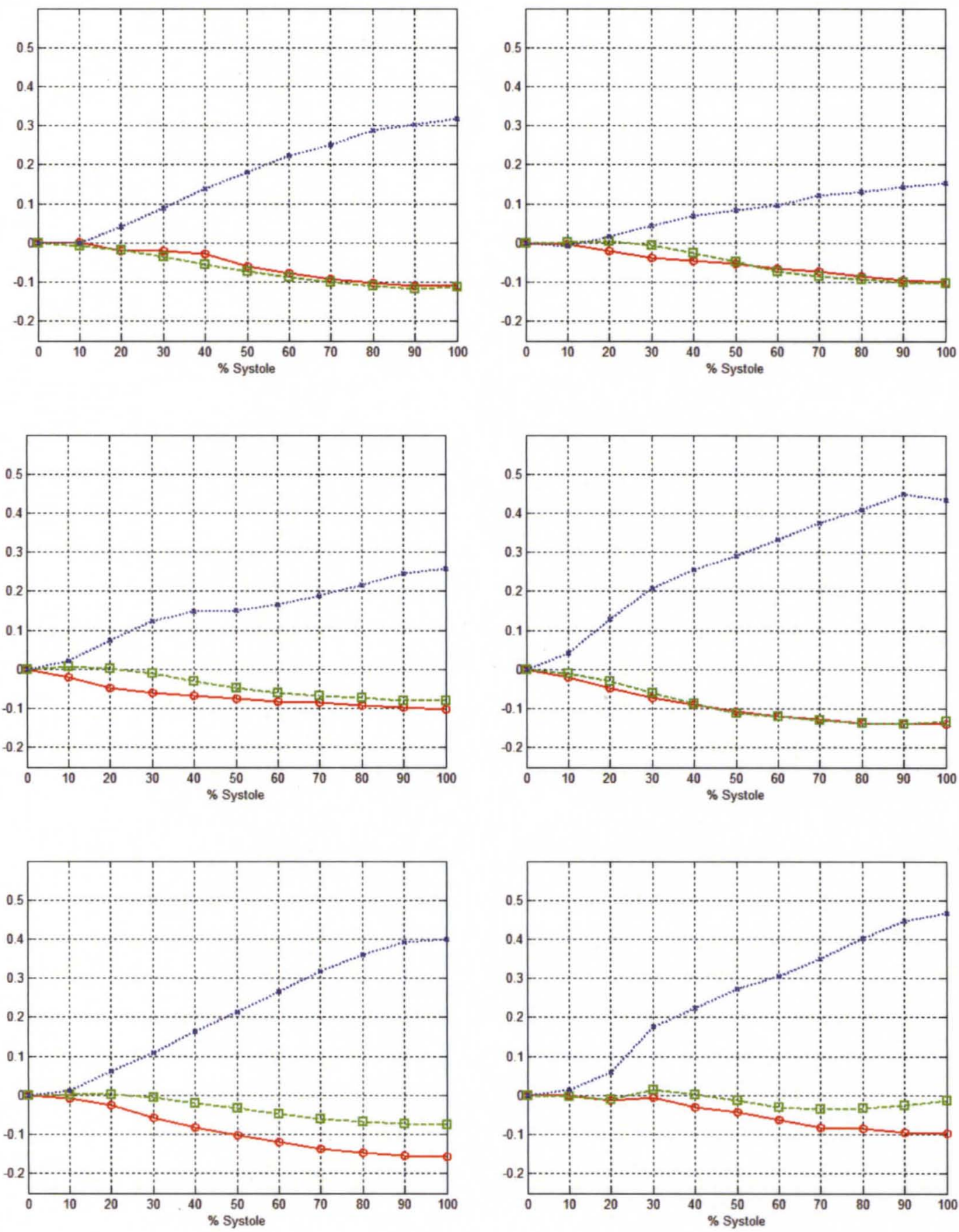

FIGURE 85 - The average radial, circumferential, and longitudinal strain curves during systole for all 7 data sets. Radial strain is shown in dotted blue line. Circumferential strain is shown in dashed green line. Longitudinal strain is shown in solid red line. Traversing from top-left to the bottom-right are results for mid-ventricular anterior (segment 7), mid-ventricular antero-septal (segment 8), mid-ventricular infero-septal (segment 9), mid-ventricular inferior (segment 10), mid-ventricular posterior (segment 11), mid-ventricular lateral (segment 12). 

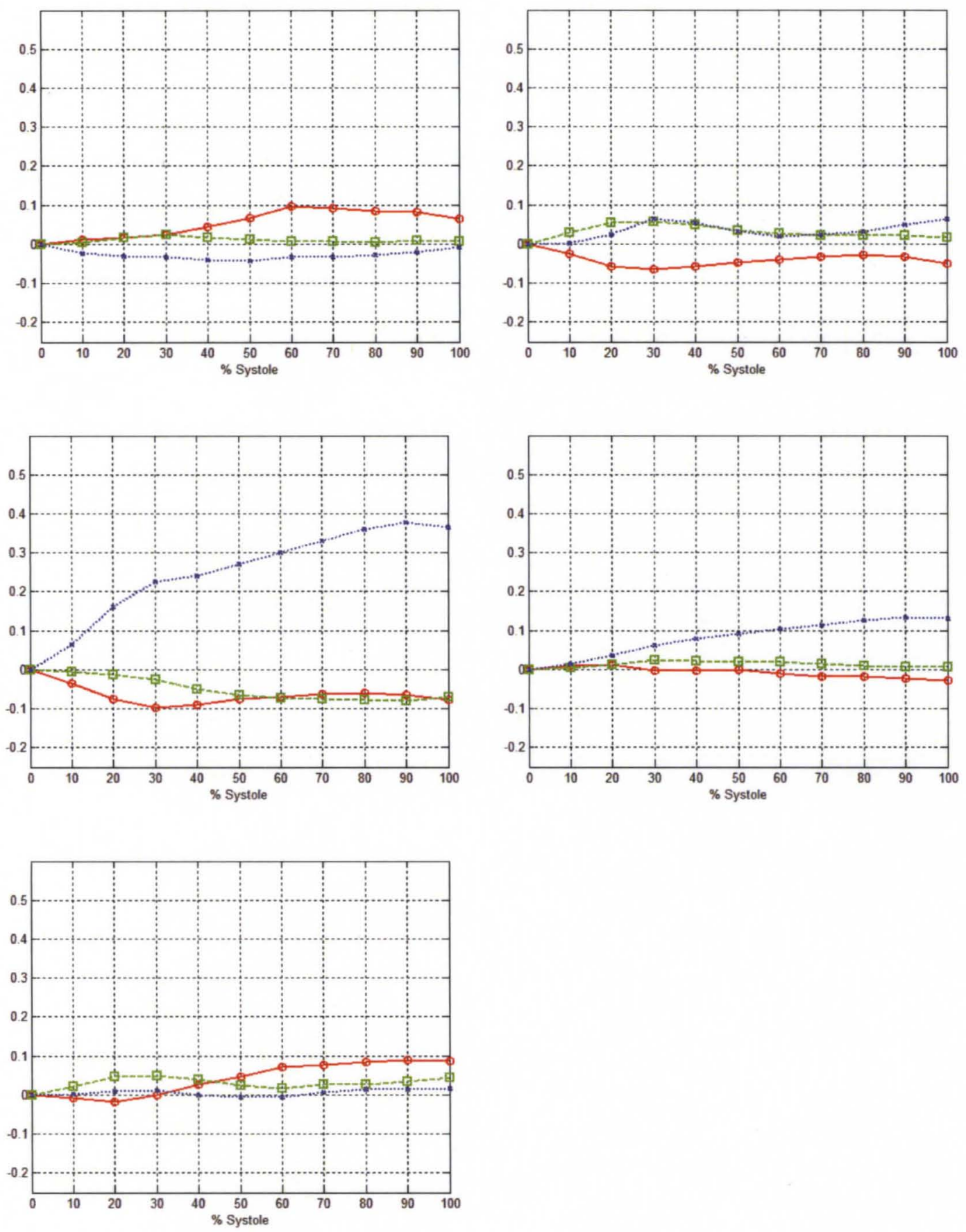

FIGURE 86 - The average radial, circumferential, and longitudinal strain curves during systole for all 7 data sets. Radial strain is shown in dotted blue line. Circumferential strain is shown in dashed green line. Longitudinal strain is shown in solid red line. Traversing from top-left to the bottom-right are results for apical anterior (segment 13), apical septal (segment 14), apical inferior (segment 15), apical lateral (segment 16), and apex (segment 17). 


\section{CHAPTER VIII CONCLUSIONS AND FUTURE DIRECTIONS}

Magnetic resonance imaging (MRI) is a highly advanced and sophisticated imaging modality for cardiac motion tracking and analysis, capable of providing 3D analysis of global and regional cardiac function with great accuracy and reproducibility. In this dissertation, the anatomy and structure of the heart, fundamentals of continuum mechanics relevant to analysis of ventricular deformations, the basics of MRI, and MRI techniques for imaging cardiac function were introduced in the first three chapters. In Chapter IV, a thorough review of existing tagged MR image analysis techniques was given. A novel multilevel B-spline technique incorporating phase-based displacement information for determining transmural deformations of the myocardium was described in Chapter $\mathrm{V}$ followed by a new MR tagging acquisition technique (OCSPAMM) in Chapter VI. In Chapter VII, a novel 3D sine wave modeling (3D SinMod) approach to automatic analysis of $3 \mathrm{D}+\mathrm{t}$ cardiac deformations from 3D CSPAMM acquisitions was proposed. The strength of this combined imaging/image analysis approach is the speed of acquisition ( 3 breathholds) for $3 \mathrm{D}+\mathrm{t}$ acquisition of CSPAMM tagged data in 3 orthogonal orientations and $5-7$ minutes for estimation of displacement and regional strains. In conclusion, the main contributions of this dissertation could be summarized as follows.

1. An updated and critical review of cardiac motion tracking methods was provided in Chapter IV. This review was also published in IEEE Transactions on Medical Imaging [75]. The MR imaging and analysis techniques surveyed were cine MRI, tagged MRI, phase contrast MRI, DENSE, and SENC. The review provides a timely update covering both MR imaging and image analysis meth- 
ods for myocardial deformation recovery. The surveyed articles are from recent journal and conference publications in the medical imaging area, including IEEE Transactions on Medical Imaging, Medical Image Analysis, IEEE Transactions on Image Processing, IEEE Transactions on Biomedical Engineering, Magnetic Resonance in Medicine, Journal of Magnetic Resonance Imaging, Journal of Magnetic Resonance, International Conference on Medical Imaging Computing and Computer Assisted Intervention, IEEE International Symposium on Biomedical Imaging, Society of Photo-Optical Instrumentation Engineers on Medical Imaging etc. The review can serve as a tutorial for new researchers entering the field.

2. A multilevel B-spline fitting method (MBS) has been proposed which incorporates phase-based displacement information for accurate calculation of $2 \mathrm{D}$ motion and strain from tagged MRI. The proposed method combines the advantages of continuity and smoothness of MBS, and makes use of phase information derived from tagged MR images. Compared to previous 2D B-spline-based deformation analysis methods, MBS has the following advantages: 1) They can simultaneously achieve a smooth deformation while accurately approximating the given data set; 2) Computationally, they are very fast; and 3) They produce more accurate deformation results. Since the tag intersections (intersections between two tag lines) can be extracted accurately and are more or less distributed evenly over the domain, MBS proved effective for cardiac motion tracking. To derive phase-based displacements, 2D HARP and SinMod analysis techniques were applied. By producing virtual tags from HARP/SinMod and calculating virtual tag intersections, more data points are obtained. It the reference frame, virtual tag lines are the isoparametric curves of an undeformed 2D B-spline model. In subsequent frames, the locations of intersections of virtual tag lines are updated with phase-based displacements. The advantage of the technique 
is that in acquiring denser myocardial displacements it uses both real and virtual tag line intersections. It is fast and more accurate than 2D HARP and SinMod tracking. Detailed description may be found in publications $[6,7]$ and in Chapter V.

3. A novel tagging pulse sequence, orthogonal complementary spatial modulation of magnetization (OCSPAMM), has been proposed which results in the same acquisition time as SPAMM for 2D deformation estimation while keeping the main advantage of CSPAMM, i.e., maintaining the tag contrast through-out the ECG cycle. Different from CSPAMM, in OCSPAMM the second tagging pulse orientation is rotated 90 degrees relative to the first one so that motion information can be obtained simultaneously in two directions. This reduces the acquisition time by a factor of two as compared to the traditional CSPAMM, in which two separate imaging sequences are applied per acquisition. Detailed description may be found in publications $[4,5]$ and in Chapter VI.

4. A novel 3D sine wave modeling (3D SinMod) approach for automatic analysis of 3D cardiac deformations has been proposed [10]. An accelerated 3D complementary spatial modulation of magnetization (CSPAMM) tagging technique [11] was used to modulate the magnetization of the myocardial tissue and to acquire 3D MR data sets of the whole-heart including three orthogonal tags within three breath-holds. With the application of CSPAMM, the effect of tag fading encountered in SPAMM tagging due to $T_{1}$ relaxation is mitigated and tag deformations can be visualized for the entire cardiac cycle, including diastolic phases. In 3D SinMod, the intensity distribution around each pixel is modeled as a cosine wave front. The principle behind 3D SinMod tracking is that both phase and frequency for each voxel are determined directly from the frequency analysis and the displacement is calculated from the quotient of phase 
difference and local frequency. The deformation fields clearly demonstrate longitudinal shortening during systole. The contraction of the LV base towards the apex as well as the torsional motion between basal and apical slices is clearly observable from the displacements. The advantages of the proposed approach are as follows.

(a) The entire framework, from data acquisition to data analysis is in the 3D domain, which permits quantification of both the in-plane and throughplane motion components.

(b) The proposed algorithm does not require acquisitions in different cardiac views (SA and LA) and makes the image acquisition planning easier for technologists in hospital.

(c) The proposed method is automatic and fast. It requires no user-interaction and the computational time for a full 3D data with $20-24$ cardiac phases is about $5-7$ minutes. The average CPU time for calculating 3D motion fields between a pair of $3 \mathrm{D}$ volumes for each of the data sets was 17.37 seconds.

Directions for future research are outlined below:

1. Extension of OCSPAMM acquisition to 3D. The current OCSPAMM technique works in 2D. The tagging pulse orientation in the second 2D SPAMM acquisition is rotated 90 degrees relative to the first one so that motion information can be obtained simultaneously in two directions. The same concept may be applied to 3D acquisition, i.e., rotating the second 3D SPAMM acquisition orthogonal to the first one prior to the subtraction in current 3D CSPAMM framework.

2. Apply the proposed 3D SinMod technique to both LV and RV deformation analysis. Cardiac analysis has focused mostly on the left ventricular myocardium, 
since LV, as the primary heart chamber, controls systemic perfusion and is responsible for pumping blood throughout the body. Compared to LV, RV has a thinner wall and more complex geometry. Besides, RV can have a large throughplane motion near the base of the ventricle (about $15 \mathrm{~mm}$ ) which is more than twice the thickness of a short-axis image plane. All these factors increase the difficulty in motion analysis. These challenges provide an opportunity for the proposed 3D SinMod technique, since it is capable of fully 3D analysis and can recover both the in-plane and through-plane deformations. As long as the 3D CSPAMM acquisition fully covers the RV, The proposed 3D SinMod technique will be applicable to RV motion analysis.

3. Extension of multilevel B-spline fitting framework (MBS)to 3D. The MBS framework is a scattered data fitting technique. It can be extended to $3 \mathrm{D}$ by finding one-to-one correspondences for both real and virtual tag line intersections at different time frames in 3D domain. The transformation represented by B-spline control points can then be obtained by minimizing the residual errors between corresponding points in two subsequent frames.

4. Investigate the $3 \mathrm{D}$ regional deformation patterns on patients with different cardiac pathologies. The proposed 3D SinMod technique has shown good results on healthy subjects. It can be used in patients to investigate patterns of abnormal wall motion for different diseases, e.g., patients with myocardial scars and heart failure.

5. 3D image segmentation technique for myocardium. In the present framework, the endocardium and epicardium contours were delineated manually. Although the motion field calculation is fully automatic and requires no user-interaction, in order to obtain motion fields, some tedious work for segmenting the contours is still necessary. 
6. An end-to-end system for fully 3D wall deformation analysis from imaging to image analysis. A full 3D cardiac wall motion analysis system mainly consists of image acquisition, post-processing, and image analysis. This dissertation focuses on the image post-processing and deformation field calculation. The 3D acquisition used 3D CSPAMM technique and all the post-processing and deformation calculation have been done offline. Ultimately, it would be ideal to develop an online acquisition and processing system that is automatic and easy to use for physicians.

In recent times, MRI tagging has seen increased applications and is becoming the gold standard for quantifying regional function. Research efforts on cardiac motion analysis from tagged MRI are devoted to developing quantitative methods for automatic, fast, and robust image analysis. With the development of more advanced MR imaging techniques and progress in hardware, research in this area is guaranteed to be exciting and to grow. 


\section{REFERENCES}

[1] J. B. Garrison, W. L. Ebert, R. E. Jenkins, S. M. Yionoulis, H. Malcom, G. A. Heyler, A. A. Shoukas, W. L. Maughan, and K. Sagawa, "Measurement of threedimensional positions and motions of large numbers of spherical radiopaque markers from biplane cineradiograms," Computers and Biomedical Research, vol. 15, no. 1, pp. 76-96, February 1982.

[2] A. F. Frangi, W. J. Niessen, and M. A. Viergever, "Three-dimensional modeling for functional analysis of cardiac images: A review," IEEE Transactions on Medical Imaging, vol. 20, no. 1, pp. 2-25, January 2001.

[3] A. A. Young, "Assessment of cardiac performance with magnetic resonance imaging," Current Cardiology Reviews, vol. 2, no. 4, pp. 271-282, November 2006.

[4] H. Wang, M. Kadbi, M. Kotys, M. Ersoy, G. P. Chatzimavroudis, R. M. Setser, S. E. Fischer, and A. A. Amini, "Orthogonal CSPAMM (OCSPAMM) MR tagging for imaging ventricular wall motion," in 33rd Annual International Conference of the IEEE Engineering in Medicine and Biology Society, August 2011.

[5] H. Wang, M. Kadbi, M. Alshaher, M. Traughber, S. E. Fischer, and A. A. Amini, "Cardiac deformation analysis from orthogonal CSPAMM (OCSPAMM) tagged MRI," in SCMR/ISMRM Jointly Sponsored Workshop - Exploring New Dimensions in Cardiovascular Flow and Motion, February 1-2 2012.

[6] H. Wang and A. A. Amini, "Accurate 2-D cardiac motion tracking using scattered data fitting incorporating phase information from MRI," in Proceedings of SPIE Medical Imaging 2010: Biomedical Applications in Molecular, Structural, and Functional Imaging, vol. 7626, February 2010.

[7] - - "Cardiac motion tracking approach with multilevel B-splines and SinMod from tagged MRI," in Proceedings of SPIE Medical Imaging 2011: Biomedical Applications in Molecular, Structural, and Functional Imaging, vol. 7965, February 2011.

[8] N. F. Osman, W. S. Kerwin, E. R. McVeigh, and J. L. Prince, "Cardiac motion tracking using CINE harmonic phase (HARP) magnetic resonance imaging," Magnetic Resonance in Medicine, vol. 42, no. 6, pp. 1048-1060, December 1999.

[9] T. Arts, F. W. Prinzen, T. Delhaas, J. Milles, A. Rossi, and P. Clarysse, "Mapping displacement and deformation of the heart with local sine wave modeling," IEEE Transactions on Medical Imaging, vol. 29, no. 5, pp. 1114-1123, May 2010. 
[10] H. Wang and A. A. Amini, "Cardiac deformation analysis using 3D sinmod from 3D CSPAMM tagged MRI," in Proceedings of SPIE Medical Imaging 2013: Biomedical Applications in Molecular, Structural, and Functional Imaging, February 2013.

[11] A. K. Rutz, S. Ryf, S. Plein, P. Boesiger, and S. Kozerke, "Accelerated wholeheart 3D CSPAMM for myocardial motion quantification," Magnetic Resonance in Medicine, vol. 59, no. 4, pp. 755-763, April 2008.

[12] Texas Heart Institute, "Heart anatomy," http://www.texasheart.org/HIC/ Anatomy/index.cfm.

[13] A. A. Amini and J. L. Prince, Measurement of Cardiac Deformations from MRI: Physical and Mathematical Models. Kluwer Academic Publishers, Dordrecht, The Netherlands, 2001.

[14] M. A. Bernstein, K. F. King, and X. J. Zhou, Handbook of MRI Pulse Sequences. Elsevier Academic Press, 2004.

[15] A. H. Aletras, S. Ding, R. S. Balaban, and W. Han, "DENSE: Displacement encoding with stimulated echoes in cardiac functional MRI," Journal of Magnetic Resonance, vol. 137, no. 1, pp. 247-252, March 1999.

[16] N. F. Osman, S. Sampath, E. Atalar, and J. L. Prince, "Imaging longitudinal cardiac strain on short-axis images using strain-encoded MRI," Magnetic Resonance in Medicine, vol. 46, no. 2, pp. 324-334, August 2001.

[17] N. J. Tustison, "Biventricular myocardial strains with anatomical NURBS models from tagged MRI," Ph.D. dissertation, Washington University in St. Louis, August 2004.

[18] World Health Organization, "Cardiovascular diseases," http://www.who.int/ entity/nmh/publications/fact_sheet_cardiovascular_en.pdf.

[19] A. H. A. S. Committee and S. S. Subcommittee, "Heart disease and stroke statistics - 2009 update," Circulation, vol. 119, no. 3, pp. e21-e181, 2009.

[20] E. Castillo, A. C. Lima, and D. A. Bluemke, "Regional myocardial function: Advances in MR imaging and analysis," Radiographics, vol. 23, pp. S127-S140, October 2003.

[21] E. Purcell, H. Torrey, and R. Pound, "Resonance absorption by nuclear magnetic moments in a solid," Physical Review, vol. 69, no. 1-2, pp. 37-38, 1946.

[22] F. Bloch, W. W. Hansen, and M. Packard, "Nuclear induction," Physical Review, vol. 69, no. 3-4, p. 127, 1946.

[23] E. M. Haacke, R. W. Brown, M. R. Thompson, and R. Venkatesan, Magnetic Resonance Imaging: Physical Principles and Sequence Design. Wiley-Liss, 1999. 
[24] Z.-P. Liang and P. C. Lauterbur, Principles of Magnetic Resonance Imaging: A Signal Processing Perspective. Wiley-IEEE Press, 1999.

[25] J. L. Prince and J. Links, Medical Imaging Signals and Systems. Prentice Hall, 2005.

[26] P. C. Lauterbur, "Image formation by induced local interactions: Examples employing nuclear magnetic resonance," Nature, vol. 242, pp. 190-191, March 1973.

[27] E. A. Zerhouni, D. M. Parish, W. J. Rogers, A. Yang, and E. P. Shapiro, "Human heart: Tagging with MR imaging-a method for noninvasive assessment of myocardial motion," Radiology, vol. 169, no. 1, pp. 59-63, October 1988.

[28] L. Axel and L. Dougherty, "MR imaging of motion with spatial modulation of magnetization," Radiology, vol. 171, no. 3, pp. 841-845, June 1989.

[29] L. Axel, A. Montillo, and D. Kim, "Tagged magnetic resonance imaging of the heart: A survey," Medical Image Analysis, vol. 9, no. 4, pp. 376-393, August 2005.

[30] F. H. Epstein, "MRI of left ventricular function," Journal of Nuclear Cardiology, vol. 14, no. 5, pp. 729-744, September/October 2007.

[31] M. Analouis, Handbook of Biomedical Engineering: Medical Imaging. CRC press, 2012.

[32] S. Ubbink, P. Bovendeerd, T. Delhaas, T. Arts, and F. van de Vosse, "Towards model-based analysis of cardiac MR tagging data: Relation between left ventricular shear strain and myofiber orientation," Medical Image Analysis, vol. 10, no. 4, pp. 632-641, August 2006.

[33] P. Yan, A. Sinusas, and J. S. Duncan, "Boundary element method-based regularization for recovering of LV deformation," Medical Image Analysis, vol. 11, no. 6, pp. 540-554, December 2007.

[34] A. Bistoquet, J. Oshinshi, and O. Skrinjar, "Left ventricular deformation recovery from cine MRI using an incompressible model," IEEE Transactions on Medical Imaging, vol. 26, no. 9, pp. 1136-1153, September 2007.

[35] — - "Myocardial deformation recovery from cine MRI using a nearly incompressible biventricular model," Medical Image Analysis, vol. 12, no. 1, pp. 69-85, February 2008.

[36] A. I. Veress, J. A. Weiss, R. D. Rabbitt, J. N. Lee, and G. T. Gullberg, "Measurement of $3 \mathrm{D}$ of left ventricular strains during diastole using image warping and untagged MRI images," Computers in Cardiology, vol. 28, pp. 165-168, 2001. 
[37] A. I. Veress, G. T. Gullberg, and J. A. Weiss, "Measurement of strain in the left ventricle during diastole with cine-MRI and deformable image registration," Journal of Biomechanical Engineering, vol. 127, no. 7, pp. 1195-1207, December 2005.

[38] D. Perperidis, R. H. Mohiaddin, and D. Rueckert, "Spatio-temporal free-form registration of cardiac MR image sequences," Medical Image Analysis, vol. 9, no. 5, pp. 441-456, October 2005.

[39] H. Sundar, H. Litt, and D. Shen, "Estimating myocardial motion by 4D image warping," Pattern Recognition, vol. 42, no. 11, pp. 2514-2526, November 2009.

[40] T. D. Nguyen, S. J. Reeves, and T. S. Denney, Jr., "On the optimality of magnetic resonance tag patterns for heart wall motion estimation," IEEE Transactions on Image Processing, vol. 12, no. 5, pp. 524-532, 2003.

[41] V. M. Pai and L. Axel, "Advances in MRI tagging techniques for determining regional myocardial strain," Current Cardiology Reports, vol. 8, no. 1, pp. 53-58, 2006 .

[42] M. L. Shehata, S. Cheng, N. F. Osman, D. A. Bluemke, and J. A. Lima, "Myocardial tissue tagging with cardiovascular magnetic resonance," Journal of Cardiovascular Magnetic Resonance, vol. 11, no. 55, pp. 1-12, December 2009.

[43] L. Axel and L. Dougherty, "Heart wall motion: improved method of spatial modulation of magnetization for MR imaging," Radiology, vol. 172, no. 2, pp. 349-350, 1989.

[44] S. E. Fischer, G. C. McKinnon, S. E. Maier, and P. Boesiger, "Improved myocardial tagging contrast," Magnetic Resonance in Medicine, vol. 30, no. 2, pp. 191-200, August 1993.

[45] N. J. Pelc, F. G. Sommer, K. C. Li, T. J. Brosnan, R. J. Herfkens, and D. R. Enzmann, "Quantitative magnetic resonance flow imaging," Magnetic Resonance Quarterly, vol. 10, no. 3, pp. 125-147, September 1994.

[46] N. J. Pelc, R. J. Herfkens, A. Shimakawa, and D. R. Enzmann, "Phase contrast cine magnetic resonance imaging," Magnetic Resonance Quarterly, vol. 7, no. 4, pp. 229-254, October 1991.

[47] T. G. Reese, D. A. Feinberg, J. Dou, and V. J. Wedeen, "Phase contrast MRI of myocardial 3D strain by encoding contiguous slices in a single shot," Magnetic Resonance in Medicine, vol. 47, no. 4, pp. 665-676, April 2002.

[48] M. Markl, B. Schneider, and J. Hennig, "Fast phase contrast cardiac magnetic resonance imaging: improved assessment and analysis of left ventricular wall motion," Journal of Magnetic Resonance Imaging; vol. 15, no. 6, pp. 642-653, June 2002. 
[49] B. Jung, M. Zaitsev, J. Hennig, and M. Markl, "Navigator gated high temporal resolution tissue phase mapping of myocardial motion," Magnetic Resonance in Medicine, vol. 55, no. 4, pp. 937-942, April 2006.

[50] M. Markl and J. Hennig, "Phase contrast MRI with improved temporal resolution by view sharing: k-space related velocity mapping properties," Magnetic Resonance Imaging, vol. 19, no. 5, pp. 669-676, June 2001.

[51] B. Jung, M. Markl, D. Föll, and J. Hennig, "Investigating myocardial motion by MRI using tissue phase mapping," European Journal of Cardio-theracic Surgery, vol. 29S, no. 1, pp. S150-S157, April 2006.

[52] B. Jung, D. Föll, P. Böttler, S. Petersen, J. Hennig, and M. Markl, "Detailed analysis of myocardial motion in volunteers and patients using high-temporalresolution MR tissue phase mapping," Journal of Magnetic Resonance Imaging, vol. 24, no. 5, pp. 1033-1039, November 2006.

[53] D. Föll, B. Jung, F. Staehle, E. Schilli, C. Bode, J. Hennig, and M. Markl, "Visualization of multidirectional regional left ventricular dynamics by hightemporal-resolution tissue phase mapping," Journal of Magnetic Resonance Imaging, vol. 29, no. 5, pp. 1043-1052, May 2009.

[54] A. H. Aletras, R. S. Balaban, and W. Han, "High-resolution strain analysis of the human heart with fast-DENSE," Journal of Magnetic Resonance, vol. 140, no. 1, pp. 41-57, September 1999.

[55] A. H. Aletras and W. Han, "Mixed echo train acquisition displacement encoding with stimulated echoes: An optimized DENSE method for in vivo functional imaging of the human heart," Magnetic Resonance in Medicine, vol. 46, no. 3, pp. 523-534, September 2001.

[56] A. H. Aletras, W. P. Ingkanisorn, C. Mancini, and A. E. Arai, "DENSE with SENSE," Journal of Magnetic Resonance, vol. 176, no. 1, pp. 99-106, September 2005.

[57] D. Kim, W. D. Gilson, C. M. Kramer, and F. H. Epstein, "Myocardial tissue tracking with two-dimensional cine displacement encoded MR imaging: development and initial evaluation," Radiology, vol. 230, no. 3, pp. 862-871, March 2004 .

[58] D. Kim, F. H. Epstein, W. D. Gilson, and L. Axel, "Increasing the signal-tonoise ratio in DENSE MRI by combining displacement-encoded echoes," Magnetic Resonance in Medicine, vol. 52, no. 1, pp. 188-192, July 2004.

[59] X. Zhong, P. A. Helm, and F. H. Epstein, "Balanced multipoint displacement encoding for DENSE MRI," Magnetic Resonance in Medicine, vol. 61, no. 6, pp. 981-988, April 2009. 
[60] B. S. Spottiswoode, X. Zhong, A. T. Hess, C. M. Kramer, E. M. Meintjes, B. M. Mayosi, and F. H. Epstein, "Tracking myocardial motion from cine DENSE images using spatiotemporal phase unwrapping and temporal fitting," IEEE Transactions on Medical Imaging, vol. 26, no. 1, pp. 15-30, January 2007.

[61] B. S. Spottiswoode, X. Zhong, C. Lorenz, B. M. Mayosi, E. M. Meintjes, and F. H. Epstein, "3D myocardial tissue tracking with slice followed cine DENSE MRI," Journal of Magnetic Resonance Imaging, vol. 27, no. 5, pp. 1019-1027, May 2008.

[62] H. Wen, K. A. Marsolo, E. E. Bennett, K. S. Kutten, R. P. Lewis, D. B. Lipps, N. D. Epstein, J. F. Plehn, and P. Croisille, "Adaptive postprocessing techniques for myocardial tissue tracking with displacement-encoded MR imaging," Radiology, vol. 246, no. 1, pp. 229-240, January 2008.

[63] J. P. Kuijer, M. B. Hofman, J. J. Zwanenburg, J. T. Marcus, A. C. van Rossum, and R. M. Heethaar, "DENSE and HARP: two views on the same technique of phase-based strain imaging," Journal of Magnetic Resonance Imaging, vol. 24, no. 6 , pp. 1432-1438, December 2006.

[64] N. F. Osman, "Detecting stiff masses using strain-encoded (SENC) imaging," Magnetic Resonance in Medicine, vol. 49, no. 3, pp. 605-608, March 2003.

[65] L. Pan, M. Stuber, D. L. Kraitchman, D. L. Fritzges, W. D. Gilson, and N. F. Osman, "Real-time imaging of regional myocardial function using fast-SENC," Magnetic Resonance in Medicine, vol. 55, no. 2, pp. 386-395, February 2006.

[66] A. Youssef, E. H. Ibrahim, G. Korosoglou, M. R. Abraham, , R. G. Weiss, and N. F. Osman, "Strain-encoding cardiovascular magnetic resonance for assessment of right-ventricular regional function," Journal of Cardiovascular Magnetic Resonance, vol. 10, no. 33, pp. 1-12, July 2008.

[67] E. H. Ibrahim, M. Stuber, A. S. Fahmy, K. Z. Abd-Elmoniem, T. Sasano, M. R. Abraham, and N. F. Osman, "Real-time MR imaging of myocardial regional function using strain-encoding (SENC) with tissue through-plane motion tracking," Journal of Magnetic Resonance Imaging, vol. 26, no. 6, pp. 14611470, December 2007.

[68] E. H. Ibrahim, M. Stuber, D. L. Kraitchman, R. G. Weiss, and N. F. Osman, "Combined functional and viability cardiac MR imaging in a single breathhold," Magnetic Resonance in Medicine, vol. 58, no. 4, pp. 843-849, October 2007.

[69] E. H. Ibrahim, R. G. Weiss, M. Stuber, A. E. Spooner, and N. F. Osman, "Identification of different heart tissues from MRI C-SENC images using an unsupervised multi-stage fuzzy clustering technique," Journal of Magnetic Resonance Imaging, vol. 28, no. 2, pp. 519-526, August 2008. 
[70] S. Sampath, N. F. Osman, and J. L. Prince, "A combined harmonic phase and strain-encoded pulse sequence for measuring three-dimentional strain," Magnetic Resonance Imaging, vol. 27, no. 1, pp. 55-61, January 2009.

[71] A. T. Hess, X. Zhong, B. S. Spottiswoode, F. H. Epstein, and E. M. Meintjes, "Myocardial 3D strain calculation by combining cine displacement encoding with stimulated echoes (DENSE) and cine strain encoding (SENC) imaging," Magnetic Resonance in Medicine, vol. 62, no. 1, pp. 77-84, July 2009.

[72] M. I. . I. P. Lab, "Strain encoded MRI (SENC)," http://miip.nileu.edu.eg/senc.

[73] E. R. McVeigh and C. Ozturk, "Imaging myocardial strain," IEEE Signal Processing Magzine, vol. 18, no. 6, pp. 44-56, November 2001.

[74] C. Ozturk, J. A. Derbyshire, and E. R. McVeigh, "Estimating motion from MRI data," Proceedings of the IEEE, vol. 91, no. 10, pp. 1627-1648, October 2003.

[75] H. Wang and A. A. Amini, "Cardiac motion and deformation recovery from MRI: A review," IEEE Transactions on Medical Imaging, vol. 30, no. 2, pp. 487-503, February 2012.

[76] A. A. Amini, Y. Chen, M. Elayyadi, and P. Radeva, "Tag surface reconstruction and tracking of myocardial beads from SPAMM-MRI with parametric B-spline surfaces," IEEE Transactions on Medical Imaging, vol. 20, no. 2, pp. 94-103, February 2001.

[77] W. S. Kerwin and J. L. Prince, "Cardiac material markers from tagged MR images," Medical Image Analysis, vol. 2, no. 4, pp. 339-353, December 1998.

[78] S. Sampath and J. L. Prince, "Automatic 3D tracking of cardiac material markers using slice-following and harmonic-phase MRI," Magnetic Resonance Imaging, vol. 25, no. 2, pp. 197-208, February 2007.

[79] J. Liang, Y. Wang, and Y. Jia, "Cardiac motion estimation from tagged MRI using 3D-HARP and NURBS volumetric model," in Proceedings of 8th Asian Conference on Computer Vision, vol. 4843, November 2007, pp. 512-521.

[80] T. McInerney and D. Terzopoulos, "Deformable models in medical image analysis: A survey," Medical Image Analysis, vol. 1, no. 2, pp. 91-108, June 1996.

[81] P. Shi, A. J. Sinusas, R. T. Constable, E. Ritman, and J. S. Duncan, "Pointtracked quantitative analysis of left ventricular surface motion from 3-D image sequences," IEEE Transactions on Medical Imaging, vol. 19, no. 1, pp. 36-50, January 2000 .

[82] P. Shi and H. Liu, "Stochastic finite element framework for simultaneous estimation of cardiac kinematic functions and material parameters," Medical Image Analysis, vol. 7, no. 4, pp. 445-464, December 2003. 
[83] V. Y. Wang, H. I. Lam, D. B. Ennis, B. R. Cowan, A. A. Young, and M. P. Nash, "Modelling passive diastolic mechanics with quantitative MRI of cardiac structure and function," Medical Image Analysis, vol. 13, no. 5, pp. 773-784, October 2009.

[84] J. Park, D. Metaxas, and L. Axel, "Analysis of left ventricular wall motion based on volumetric deformable models and MRI-SPAMM," Medical Image Analysis, vol. 1, no. 1, pp. 53-71, March 1996.

[85] N. J. Tustison, V. G. Dávila-Román, and A. A. Amini, "Myocardial kinematics from tagged MRI based on a 4-D B-spline model," IEEE Transactions on Biomedical Engineering, vol. 50, no. 8, pp. 1038-1040, August 2003.

[86] X. Papademetris, A. J. Sinusas, P. Dione, R. T. Constable, and J. S. Duncan, "Estimation of 3-D left ventricular deformation from medical images using biomechnical models," IEEE Transactions on Medical Imaging, vol. 21, no. 7, pp. 786-800, July 2002.

[87] P. Clarysse, C. Basset, L. Khouas, P. Croisille, D. Friboulet, C. Odet, and I. E. Magnin, "Two-dimensional spatial and temporal displacement and deformation field fitting from cardiac magnetic resonance tagging," Medical Image Analysis, vol. 4, no. 3, pp. 253-268, September 2000.

[88] I. Haber, D. Metaxas, and L. Axel, "Three-dimensional motion reconstruction and analysis of the right ventricle using tagged MRI," Medical Image Analysis, vol. 4, no. 4, pp. 335-355, December 2000.

[89] Z. Hu, D. Metaxas, and L. Axel, "In vivo strain and stress estimation of the heart left and right ventricles from MRI images," Medical Image Analysis, vol. 7, no. 4, pp. 435-444, December 2003.

[90] - - "Left ventricle composite material model for stress-strain analysis," in Proceedings of the 2003 International Conference on Surgery Simulation and Soft Tissue Modeling, June 2003, pp. 218-229.

[91] A. A. Amini, Y. Chen, R. W. Curwen, V. Mani, and J. Sun, "Coupled B-snake grids and constrained thin-plate splines for analysis of 2-D tissue deformations from tagged MRI," IEEE Transactions on Medical Imaging, vol. 17, no. 3, pp. 344-356, June 1998.

[92] D. Suter and F. Chen, "Left ventricular motion reconstruction based on elastic vector splines," IEEE Transactions on Medical Imaging, vol. 19, no. 4, pp. 295-305, April 2000.

[93] Y. Wang, Y. Chen, and A. A. Amini, "Fast LV motion estimation using subspace approximation techniques," IEEE Transactions on Medical Imaging, vol. 20, no. 6, pp. 499-513, June 2001. 
[94] P. Radeva, A. A. Amini, and J. Huang, "Deformable B-solids and implicit snakes for 3D localization and tracking of SPAMM MRI data," Computer Vision and Image Understanding, vol. 66, no. 2, pp. 163-178, May 1997.

[95] J. Huang, D. Abendschein, V. G. Dávila-Román, and A. A. Amini, "Spatiotemporal tracking of myocardial deformations with a 4-D B-spline model from tagged MRI," IEEE Transactions on Medical Imaging, vol. 18, no. 10, pp. 957972, October 1999.

[96] C. Ozturk and E. R. McVeigh, "Four-dimensional B-spline based motion analysis of tagged MR images: introduction and in vivo validation," Physics in Medicine and Biology, vol. 45, no. 6, pp. 1683-1702, June 2000.

[97] N. J. Tustison and A. A. Amini, "Biventricular myocardial strains via nonrigid registration of anatomical NURBS models," IEEE Transactions on Medical Imaging, vol. 25, no. 1, pp. 94-112, January 2006.

[98] J. Chen, N. J. Tustison, and A. A. Amini, "Accurate recovery of 4-D left ventricular deformations using volumetric B-splines incorporating phase based displacement estimates," in Proceedings of SPIE Medical Imaging, vol. 6143, February 2006.

[99] X. Deng and T. S. Denney, Jr., "Three-dimensional myocardial strain reconstruction from tagged MRI using a cylindrical B-spline model," IEEE Transactions on Medical Imaging, vol. 23, no. 7, pp. 861-867, July 2004.

[100] - "Combined tag tracking and strain reconstruction from tagged cardiac MR images without user-defined myocardial contours," Journal of Magnetic Resonance Imaging, vol. 21, no. 1, pp. 12-22, January 2005.

[101] A. A. Young, "Model tags: direct three-dimensional tracking of heart wall motion from tagged magnetic resonance images," Medical Image Analysis, vol. 3, no. 4, pp. 361-372, December 1999.

[102] A. A. Young, D. L. Kraitchman, L. Dougherty, and L. Axel, "Tracking and finite-element analysis of stripe deformation in magnetic resonance tagging," IEEE Transactions on Medical Imaging, vol. 14, no. 5, pp. 413-421, 1995.

[103] J. L. Prince and E. R. McVeigh, "Motion estimation from tagged MR image sequences," IEEE Transactions on Medical Imaging, vol. 11, no. 2, pp. 238-249, 1992.

[104] L. Dougherty, J. C. Asmuth, A. S. Blom, L. Axel, and R. Kumar, "Validation of an optical flow method for tag displacement estimation," IEEE Transactions on Medical Imaging, vol. 18, no. 4, pp. 359-363, April 1999.

[105] C. Xu, J. J. Pilla, G. Isaac, J. H. Gorman, A. S. Blom, R. C. Gorman, Z. Ling, and L. Dougherty, "Deformation analysis of 3D tagged cardiac images using an 
optical flow method," Journal of Cardiovascular Magnetic Resonance, vol. 12, no. 19, March 2010.

[106] D. Fleet and A. Jepson, "Computation of component image velocity from local phase information," International Journal of Computer Vision, vol. 5, pp. 77104, 1990.

[107] L. Florack, H. V. Assen, and A. Suinesiaputra, "Dense multiscale motion extraction from cardiac cine MR tagging using HARP technology," in ICCV workshop on MMBIA, October 2007, pp. 372-375.

[108] H. C. van Assen, L. M. Florack, J. J. Westenberg, and B. M. ter Haar Romeny, "Tuple image multi-scale optical flow for detailed cardiac motion extraction: Application to left ventricle rotation analysis," in Proceedings of the MICCAI Workshop on Analysis of Functional Medical Images, September 2008, pp. $73-$ 80 .

[109] T. Mäkelä, P. Clarysse, O. Sipilä, N. Pauna, Q. C. Pham, T. Katila, and I. E. Magnin, "A review of cardiac image registration methods," IEEE Transactions on Medical Imaging, vol. 21, no. 9, pp. 1011-1021, September 2002.

[110] D. Rueckert, L. I. Sonoda, C. Hayes, D. L. G. Hill, M. O. Leach, and D. J. Hawkes, "Nonrigid registration using free-form deformations: Application to breast MR images," IEEE Transactions on Medical Imaging, vol. 18, no. 8, pp. 712-721, August 1999.

[111] R. Chandrashekara, R. H. Mohiaddin, and D. Rueckert, "Analysis of 3-D myocardial motion in tagged MR images using nonrigid image registration," IEEE Transactions on Medical Imaging, vol. 23, no. 10, pp. 1245-1250, October 2004.

[112] — - "Cardiac motion tracking in tagged MR images using a 4D B-spline motion model and nonrigid image registration," in IEEE International Symposium on Biomedical Imaging: Nano to Macro, vol. 1, April 2004, pp. 468-471.

[113] N. Rougon, C. Petitjean, F. Prêteux, P. Cluzel, and P. Grenier, "A non-rigid registraion approach for quantifying myocardial contraction in tagged MRI using generalized information measures," Medical Image Analysis, vol. 9, no. 4, pp. 353-375, August 2005.

[114] J. B. A. Maintz and M. A. Viergever, "A survey of medical image registration," Medical Image Analysis, vol. 2, no. 1, pp. 1-36, March 1998.

[115] N. F. Osman, E. R. McVeigh, and J. L. Prince, "Imaging heart motion using harmonic phase MRI," IEEE Transactions on Medical Imaging, vol. 19, no. 3, pp. 186-202, March 2000.

[116] N. F. Osman and J. L. Prince, "Visualizing myocardial function using HARP MRI," Physics in Medicine and Biology, vol. 45, no. 6, pp. 1665-1682, 2000. 
[117] — - "Regenerating MR tagged images using harmonic phase (HARP) methods," IEEE Transactions on Biomedical Engineering, vol. 51, no. 8, pp. 14281433, August 2004.

[118] S. Ryf, J. Tsao, J. Schwitter, A. Stuessi, and P. Boesiger, "Peak-combination HARP: a method to correct for phase error in HARP," Journal of Magnetic Resonance Imaging, vol. 20, no. 5, pp. 874-880, November 2004.

[119] X. Liu, E. Murano, M. Stone, and J. Prince, "HARP tracking refinement using seeded region growing," in IEEE International Symposium on Biomedical Imaging: Nano to Macro, April 2007, pp. 372-375.

[120] X. Liu, Y. Bai, and J. L. Prince, "Shortest path refinement for HARP motion tracking," Proceedings of SPIE Medical Imaging, vol. 7259, February 2009.

[121] S. Sampath, J. A. Derbyshire, N. F. Osman, E. Atalar, and J. L. Prince, "Real-time imaging of cardiac strain using ultra fastHARP sequence," in Proc. ISMRM 9th Scientific Meeting Exhibition 2001, April 2001.

[122] S. Sampath, J. A. Derbyshire, E. Atalar, N. F. Osman, and J. L. Prince, "Realtime imaging of two-dimensional cardiac strain using a harmonic phase magnetic resonance imaging(HARP-MRI) pulse sequence," Magnetic Resonance in Medicine, vol. 50, no. 1, pp. 154-163, July 2003.

[123] K. Z. Abd-Elmoniem, S. Sampath, N. F. Osman, and J. L. Prince, "Real-time monitoring of cardiac regional function using fastHARP MRI and region-ofinterest reconstruction," IEEE Transactions on Biomedical Engineering, vol. 54, no. 9, pp. 1650-1656, September 2007.

[124] L. Pan, J. L. Prince, J. A. C. Lima, and N. F. Osman, "Fast tracking of cardiac motion using 3D-HARP," IEEE Transactions on Biomedical Engineering, vol. 52, no. 8, pp. 1425-1435, August 2005.

[125] S. Ryf, M. A. Spiegel, M. Gerber, and P. Boesiger, "Myocardial tagging with 3D-CSPAMM," Journal of Magnetic Resonance Imaging, vol. 16, no. 3, pp. 320-325, September 2002.

[126] K. Z. Abd-Elmoniem, N. F. Osman, J. L. Prince, and M. Stuber, "Threedimensional magnetic resonance myocardial motion tracking from a single image plane," Magnetic Resonance in Medicine, vol. 58, no. 1, pp. 92-102, July 2007.

[127] S. E. Fischer, G. C. McKinnon, M. B. Scheidegger, W. Prins, D. Maier, and P. Boesiger, "True myocardial motion tracking," Magnetic Resonance in Medicine, vol. 31, no. 4, pp. 401-413, April 1994.

[128] K. Z. Abd-Elmoniem, M. Stuber, and J. L. Prince, "Direct three-dimentional myocardial strain tensor quantification and tracking using zHARP," Medical Image Analysis, vol. 12, no. 6, pp. 778-786, December 2008. 
[129] S. Sampath, N. F. Osman, and J. L. Prince, "Imaging the evolution of threedimentional myocardial strains using a fast MR imaging technique," in IEEE International Symposium on Biomedical Imaging: Nano to Macro, vol. 1, April 2004, pp. 624-627.

[130] Y. Chen and A. A. Amini, "A MAP framework for tag line detection in SPAMM data using Markov random fields on the B-spline solid," IEEE Transactions on Medical Imaging, vol. 21, no. 9, pp. 1110-1122, September 2002.

[131] T. Chen and L. Axel, "Using Gabor filter banks and temporal-spatial constraints to compute 3D myocardium strain," in Proceedings of IEEE 2006 International Conference of the Engineering in Medicine and Biology Society, vol. 1, August 2006, pp. 4755-4758.

[132] A. Montillo, D. N. Metaxas, and L. Axel, "Extracting tissue deformation using Gabor filter banks," in Proceedings of SPIE Medical Imaging 2004: Physiology, Function, and Structure from Medical Images, vol. 5369, February 2004.

[133] Z. Qian, D. N. Metaxas, and L. Axel, "Extraction and tracking of MRI tagging sheets using a 3D Gabor filter bank," in Proceedings of IEEE 2006 International Conference of the Engineering in Medicine and Biology Society, vol. 1, August 2006, pp. 711-714.

[134] T. Chen, X. Wang, S. Chung, D. Metaxas, and L. Axel, "Automated 3D motion tracking using Gabor filter bank, robust point matching, and deformable models," IEEE Transactions on Medical Imaging, vol. 29, no. 1, pp. 1-11, January 2010 .

[135] Z. Qian, D. N. Metaxas, and L. Axel, "Non-tracking-based 2D strain estimation in tagged MRI," in IEEE International Symposium on Biomedical Imaging: Nano to Macro, vol. 1, May 2008, pp. 711-714.

[136] C. C. Moore, C. H. Lugo-Olivieri, E. R. McVeigh, and E. A. Zerhouni, "Threedimensional systolic strain patterns in the normal human left ventricle: Characterization with tagged MR imaging," Radiology, vol. 214, no. 2, pp. 453 466, February 2000.

[137] C. C. Moore, E. R. McVeigh, and E. A. Zerhouni, "Quantitative tagged magnetic resonance imaging of the normal human left ventricle," Top Magnetic Resonance Imaging, vol. 11, no. 6, pp. 359-371, 2000.

[138] P. Clarysse, M. Han, P. Croisille, and I. E. Magnin, "Exploratory analysis of the spatio-temporal deformation of the myocardium during systole from tagged MRI," IEEE Transactions on Biomedical Engineering, vol. 49, no. 11, pp. 13281339, November 2002.

[139] E. W. Remme, A. A. Young, K. F. Augenstein, B. Cowan, and P. J. Hunter, "Extraction and quantification of left ventricular deformation modes," IEEE 
Transactions on Biomedical Engineering, vol. 51, no. 11, pp. 1923-1931, November 2004 .

[140] B. P. Cupps, P. Moustakidis, B. J. Pomerantz, G. Vedala, R. P. Scheri, N. T. Kouchoukos, V. G. Davila-Roman, and M. K. Pasque, "Severe aortic insufficiency and normal systolic function: determining regional left ventricular wall stress by finite-element analysis," Annals of Thoracic Surgery, vol. 76, no. 3, pp. 668-675, September 2003.

[141] P. Moustakidis, B. P. Cupps, B. J. Pomerantz, R. P. Scheri, H. S. Maniar, A. M. Kates, R. J. Gropler, M. K. Pasque, and T. M. Sundt, 3rd., "Noninvasive, quantitative assessment of left ventricular function in ischemic cardiomyopathy," Journal of Surgical Research, vol. 116, no. 2, pp. 187-196, February 2004.

[142] D. Bree, J. R. Wollmuth, B. P. Cupps, M. D. Krock, A. Howells, J. Rogers, N. Moazami, and M. K. Pasque, "Low-dose dobutamine tissue-tagged MRI with 3D strain analysis allows assessment of myocardial viability in patients with ischemic cardiomyopathy," Circulation, vol. 114(1 Suppl), no. I33-I36, July 2006 .

[143] H. S. Maniar, B. P. Cupps, D. D. Potter, P. Moustakidis, C. J. Camillo, C. M. Chu, M. K. Pasque, and T. M. Sundt, 3rd., "Ventricular function after coronary artery bypass grafting: evaluation by magnetic resonance imaging and myocardial strain analysis," Journal of Thoracic and Cardiovascular Surgery, vol. 128, no. 1, pp. 76-82, July 2004.

[144] D. D. Potter, P. A. Araoz, K. P. McGee, W. S. Harmsen, J. N. Mandrekar, and T. M. Sundt, 3rd., "Low-dose dobutamine cardiac magnetic resonance imaging with myocardial strain analysis predicts myocardial recoverability after coronary artery bypass grafting," Journal of Thoracic and Cardiovascular Surgery, vol. 135 , no. 6 , pp. 1342-1347, June 2008.

[145] M. A. Guttman, J. L. Prince, and E. R. McVeigh, "Tag and contour detection in tagged MR images of the left ventricle," IEEE Transactions on Medical Imaging, vol. 13, no. 1, pp. 74-88, 1994.

[146] T. S. Denney, Jr., "Estimation and detection of myocardial tags in MR image without user-defined myocardial contours," IEEE Transactions on Medical Imaging, vol. 18, no. 4, pp. 330-344, April 1999.

[147] S. Lee, G. Wolberg, and S. Y. Shin, "Scattered data interpolation with multilevel B-splines," IEEE Transanctions on Visualization and Computer Graphics, vol. 3, no. 3, pp. 228-244, 1997.

[148] T. Arts, W. C. Hunter, A. Douglas, A. M. Muijtjens, and R. S. Reneman, "Description of the deformation of the left ventricle by a kinematic model," Journal of Biomechanics, vol. 25, no. 10, pp. 1119-1127, 1992. 
[149] M. Ersoy, M. Kotys, X. Zhou, and R. M. Setser, "A left ventricular motion phantom for cardiac MRI," in Biomedical Engineering Society (BMES) 2010 Annual Meeting, October 2010.

[150] M. D. Cerqueira, N. J. Weissman, V. Dilsizian, A. K. Jacobs, S. Kaul, W. K. Laskey, D. J. Pennell, J. A. Rumberger, T. Ryan, and M. S. Verani, "Standardized myocardial segmentation and nomenclature for tomographic imaging of the heart: A statement for healthcare professionals from the cardiac imaging committee of the council on clinical cardiology of the american heart association," Circulation, vol. 105, pp. 539-542, 2002.

[151] S. S. Klein, T. P. Graham, Jr., and C. H. Lorenz, "Noninvasive delineation of normal right ventricular contractile motion with magnetic resonance imaging myocardial tagging," Annals of Biomedical Engineering, vol. 26, no. 5, pp. 756763, September-October 1998. 


\section{CURRICULUM VITAE}

\section{CONTACT INFORMATION}

\section{Hui Wang}

Lutz Hall, Room 308

Electrical and Computer Engineering Department

University of Louisville, Louisville, KY 40292

E-mail: hui.wang@louisville.edu

\section{RESEARCH INTERESTS}

Medical Imaging, Cardiac Motion Analysis, MR Pulse Sequence Programming.

\section{EDUCATION}

University of Louisville, Louisville, KY, USA

Ph.D., Electrical and Computer Engineering, November 2012. GPA: 3.97/4.0

- Thesis Topic: MR Imaging of Left-Ventricular Function: Novel Image Acquisition and Analysis Techniques

- Advisor: Professor Amir A. Amini

- Area of Study: Medical Imaging and Image Processing

Shanghai Jiao Tong University, Shanghai, China

M.S., Department of Automation, March 2007

- Thesis Topic: Image Restoration Based on BP Neural Network

- Advisor: Professor Jie Yang

- Area of Study: Image Processing and Pattern Recognition

East China University of Science and Technology, Shanghai, China

B.S.,Department of Automation, June 2004

- Area of Study: Automatic Control 


\section{RESEARCH EXPERIENCE}

University of Louisville, Louisville, KY

\section{Research Assistant}

- Proposed a novel 3D sine wave modeling (3D SinMod) approach for automatic analysis of 3D cardiac deformations.

In the proposed 3D SinMod technique, the intensity distribution around each pixel is modeled as a cosine wave front. The principle behind 3D SinMod tracking is that both phase and frequency for each voxel are determined directly from the frequency analysis and the displacement is calculated from the quotient of phase difference and local frequency. The entire framework, from data acquisition to data analysis is in the 3D domain, which permits quantification of both the in-plane and through-plane motion components. The proposed method is automatic and fast. It requires no user-interaction and the computational time for a full 3D data with $20-24$ cardiac phases is about $5-7$ minutes. The average CPU time for calculating 3D motion fields between a pair of $3 \mathrm{D}$ volumes for each of the data sets was 17.37 seconds.

- Developed a fast and accurate cardiac motion tracking method using hierarchical B-splines incorporating phase information.

A multilevel B-spline registration framework is proposed to integrate displacement information obtained from tagged images in the spatial domain with displacement information obtained from spectral peaks in the frequency domain in order to improve the accuracy of motion tracking. It combines the advantages of continuity and smoothness of MBS, and makes use of phase information derived from tagged MR images.

- Implemented two local phase based methods in the estimation of cardiac motion from MR images: HARP and SinMod.

Rather than working on tagged images directly, both HARP and SinMod techniques use phase information in the frequency domain. Tagged MRI gives rise to spectral peaks in k-space, each spectral peak containing information about motion in a particular direction. The inverse Fourier transform of a single peak, extracted using a bandpass filter, is a complex image whose phase is linearly proportional to a directional component of the true motion. The principle of HARP tracking is that the harmonic phase of a material point remains constant as it moves during the cardiac cycle. The principle behind SinMod is that both phase and frequency for each pixel are determined directly from the frequency analysis and the displacement is calculated from the ratio of phase difference and local frequency. 
Philips Medical Systems Inc., Cleveland, OH

MR Clinical Scientist Intern

April 2011- May 2012

- Proposed and implemented a novel MR pulse sequences (OCSPAMM) for cardiac motion tracking on Philips platform.

A novel pulse sequence, termed Orthogonal Complementary SPAtial Modulation of Magnetization (OCSPAMM), was proposed which reduces the acquisition time by half for $2 \mathrm{D}$ deformation estimation while keeping the advantage of CSPAMM with high tagging contrast. Different from CSPAMM, the second tagging gradient orientation in OCSPAMM is rotated 90 degrees relative to the first one so that the motion information can be obtained simultaneously in two directions.

- Assisted teaching two Philips clinical science courses: sequence development mode (SDM) and pulse programming environment (PPE).

SDM is an investigational tool for prototyping non-product pulse programs. System architecture, protocol definition file (PDF), and measurement program file (MPF) are introduced in SDM. Before calculated objects and attributes are sent to the spectrometer, SDM can be used to interrupt the process and modify them. I taught several lectures including SDM workflow, graphic viewer (GVE), and dynamic scanning.

The PPE course covers scanner simulator, development tools, and all source codes. I taught program making and compiling process as well as the patching tools. In addition to the lectures, I made some examples (High-speed STEAM programming and help file example) so that collaborators can develop a practical understanding of the programming environment. As a teaching assistant, I helped several groups to analyze, write, and debug source codes for their class projects. They all finished their codes and left the class with their patch ready to be run at the scanner.

Shanghai Jiao Tong University, Shanghai, China

Research Assistant

September 2004-March 2007

- Developed an algorithm and software for image restoration using Back-Propagation Neural Network (BPNN).

BPNN is a supervised learning method using both training data sets and testing data sets. In the training stage, each neuron in one layer is connected to every neuron in the next layer. The network functions are as follows: Each neuron receives a signal from the neurons in the previous layer, and each of those signals is multiplied by a separate weight value. The weighted inputs are summed 
and passed through a limiting function which scales the output to a fixed range of values. The output of the limiter is then broadcast to all of the neurons in the next layer. To use the network to solve a problem, the input values are applied to the inputs of the first layer, allow the signals to propagate through the network, and read the output values.

- Developed a blurring description software using $\mathrm{C}++$.

\section{BOOK CHAPTERS}

1. Hui Wang and Amir A. Amini. Cardiac MRI for Recovery of Myocardial Deformations: Imaging and Modeling. Chapter 11 of Handbook of Biomedical Engineering: Medical Imaging, M. Analouis, (Ed.), CRC press, 2012.

2. Hui Wang and Amir A. Amini. Tagging Analysis. Chapter 10 of Heart $\mathrm{Me}$ chanics: Magnetic Resonance Imaging, S Ibrahim (Ed.), Taylor \& Francis, 2012 .

\section{JOURNAL PUBLICATIONS}

1. Hui Wang and Amir A. Amini. Cardiac Motion Tracking Methods from MRI: A Review. IEEE Transactions on Medical Imaging. 31(2), pp487-503, Feb. 2012.

2. Hui Wang, Jie Yang, Ming Li, Nian Cai. An Image Restoration Method Based on Neural Network. Infrared and Laser Engineering Supplement. 2006 (EI) (In Chinese)

3. Ming Li, Jie Yang, Hui Wang, Nian Cai, A Novel Color Image Restoration Method: Sliding Window Based Support Vector Regression Algorithm. Infrared and Laser Engineering Supplement, 2006 (EI) (In Chinese)

\section{CONFERENCE PUBLICATIONS}

1. Hui Wang and Amir A. Amini. Cardiac Deformation Analysis Using 3D SinMod from 3D CSPAMM Tagged MRI. In: Proceedings of the SPIE Medical Imaging 2013: Biomedical Applications in Molecular, Structural, and Functional Imaging, Orlando, FL, USA. February 9-14, 2013. (Accepted).

2. Mo Kadbi, Hui Wang, MJ Negahdar, Lizette Warner, Melanie Traughber, Peter Martin, Jens Heidenreich, Amir Amini. A Novel Phase-Corrected 3D Cine Ultra-Short TE (UTE) Phase-Contrast MRI Technique. Annual International Conference of the IEEE Engineering in Medicine and Biology Society (EMBC12), San Diego, CA. August 28th - Sep. 1st, 2012.

3. Hui Wang, Mo Kadbi, Melanie Traughber, Amir Amini. Choice of Bandpass Filter on Accuracy of Myocardial Deformation Recovery With SinMod. 
The 20th Annual Meeting of International Society of Magnetic Resonance in Medicine, Melbourne, Australia, May 5-11, 2012.

4. Mo Kadbi, Hui Wang, Lizette Warner, Jens Heidenreich, Andrea Yancey, Motaz Alshaher, Melanie Traughber, Amir Amini. Effect of Trajectory Delay in Cine Ultra-Short TE Phase-Contrast MR Imaging of the Carotid Bifurcation. The 20th Annual Meeting of International Society of Magnetic Resonance in Medicine, Melbourne, Australia, May 5-11, 2012.

5. Mo Kadbi, Hui Wang, Jens Heidenreich, Andrea Yancey, Motaz Alshaher, Melanie Traughber, Amir Amini. 3D Cine Ultra-Short TE (UTE) Phase Contrast Imaging in Carotid Artery: Comparison with Conventional Technique. The 20th Annual Meeting of International Society of Magnetic Resonance in Medicine, Melbourne, Australia, May 5-11, 2012.

6. Hui Wang, Mo Kadbi, Motaz Alshaher, Melanie Traughber, Stefan E. Fischer, Amir A. Amini. Cardiac Deformation Analysis from Orthogonal CSPAMM (OCSPAMM) Tagged MRI. SCMR/ISMRM Jointly Sponsored Workshop - Exploring New Dimensions in Cardiovascular Flow and Motion, Orlando, FL, February 1-2, 2012. (Oral Presentation).

7. Mohammadreza Negahdar, Hui Wang, Amir A. Amini. A Novel Optical Flow Method for Myocardial Deformation Analysis from Tagged MRI. SCMR/ISMRM Jointly Sponsored Workshop - Exploring New Dimensions in Cardiovascular Flow and Motion, Orlando, FL, February 1-2, 2012.

8. Hui Wang, Mo Kadbi, Melanie Traughber, Mehmet Ersoy, George P. Chatzimavroudis, Randolph M. Setser, Stefan E. Fischer, Amir A. Amini. Orthogonal CSPAMM (OCSPAMM) MR Tagging for Imaging Ventricular Wall Motion, 33rd Annual International Conference of the IEEE Engineering in Medicine and Biology Society (EMBC11), Boston, MA. August 30th - Sep. 3rd, 2011.(Oral Presentation).

9. Mo Kadbi, Hui Wang, Mohammadjavad Negahdar, Melanie Traughber, Amir Amini, Stefan Fischer. A Real-Time Cine Late Gadolinium Enhancement Imaging Method at 3T. The 19th Annual Meeting of International Society of Magnetic Resonance in Medicine, Montreal, Canada, May 7-13, 2011.

10. Hui Wang and Amir A. Amini. Cardiac Motion Tracking with Multilevel BSplines and SinMod from Tagged MRI. In: Proceedings of the SPIE Medical Imaging 2011: Biomedical Applications in Molecular, Structural, and Functional Imaging, Orlando, FL, USA. February 12-17, 2011. (Honorable Mention Poster Award).

11. Hui Wang and Amir A. Amini. A Global Phase Unwrapping Method for Cardiac Motion Tracking Using Tagged MRI. The 38th Annual Meeting of the North American Society for Cardiovascular Imaging (NASCI), Seattle, WA, USA. October 3-5, 2010. 
12. Hui Wang and Amir A. Amini. Accurate 2-D Cardiac Motion Tracking Using Scattered Data Fitting Incorporating Phase Information from MRI. In: Proceedings of the SPIE Medical Imaging 2010: Biomedical Applications in Molecular, Structural, and Functional Imaging, San Diego, CA, USA. February 13-18, 2010. (Oral Presentation).

13. Hui Wang, Nian Cai, Ming Li, Jie Yang. Local-Information-based Blind Image Restoration Algorithm Using a MLP. International Conference on Adaptive and Natural Computing Algorithms, Warsaw, Poland, 2007, LNCS.

\section{SERVICE}

- Journal Reviewer

- IEEE Transactions on Medical Imaging

-- Medical Physics

- Computerized Medical Imaging and Graphics

- International Journal of Radiation Oncology, Biology, Physics

- Conference Reviewer

- International Conference on Medical Image Computing and Computer Assisted Intervention (MICCAI)

- SPIE Conference on Biomedical Applications in Molecular Structural, and Functional Imaging

- International Symposium on Biomedical Imaging (ISBI)

- Information Processing in Medical Imaging (IMPI)

- Volunteer at the International Workshop on Medical Imaging and Augmented Reality (MIAR2006), Shanghai, August 17 - 18, 2006.

- Student member of SPIE, ISMRM, IEEE, EMBS

\section{PROFESSIONAL TRAINING}

- Kitware Developers Training Week (VTK, ITK, CMake), Clifton Park, NY, November 3-6, 2008.

- Philips clinical science training, including Sequence Develop Mode (SDM), Pulse Programming Environment (PPE), and Data Handling, Cleveland, OH, 2010.

\section{$\underline{\text { SKILLS }}$}

- Strong background in MR physics and Image Processing.

- Proficient in Philips Pulse Programming, Matlab, C, C++. 
- Tools: Matlab Image Processing Toolbox, ITK, VTK, QT, CMake, IATEX.

- Hands-on experience in MRI Pulse Sequence Programming and Image Analysis.

- Languages: English (Fluent) and Chinese (Native).

\section{AWARDS}

- Honorable Mention Poster in the SPIE Medical Imaging Symposium for the paper Cardiac Motion Tracking with Multilevel B-Splines and SinMod from Tagged MRI, Orlando, FL, USA. February 12-17, 2011.

- Grosscurth University Fellowship, University of Louisville, 2007-2009.

- Excellent Graduate Student of Shanghai, China, 2007.

- First Prize of Social Service Project, Shanghai Jiao Tong University, 2005.

- Excellent Undergraduate Student of Shanghai, China, 2004. 